\title{
Large Amplitude Oscillatory Shear (LAOS) studies of fibrin and whole blood clots
}

\author{
Lamer, Tunde F.
}

How to cite:

Lamer, Tunde F. (2018) Large Amplitude Oscillatory Shear (LAOS) studies of fibrin and whole blood clots. Doctoral thesis, Swansea University.

http://cronfa.swan.ac.uk/Record/cronfa46063

Use policy:

This item is brought to you by Swansea University. Any person downloading material is agreeing to abide by the terms of the repository licence: copies of full text items may be used or reproduced in any format or medium, without prior permission for personal research or study, educational or non-commercial purposes only. The copyright for any work remains with the original author unless otherwise specified. The full-text must not be sold in any format or medium without the formal permission of the copyright holder. Permission for multiple reproductions should be obtained from the original author.

Authors are personally responsible for adhering to copyright and publisher restrictions when uploading content to the repository.

Please link to the metadata record in the Swansea University repository, Cronfa (link given in the citation reference above.)

http://www.swansea.ac.uk/library/researchsupport/ris-support/ 


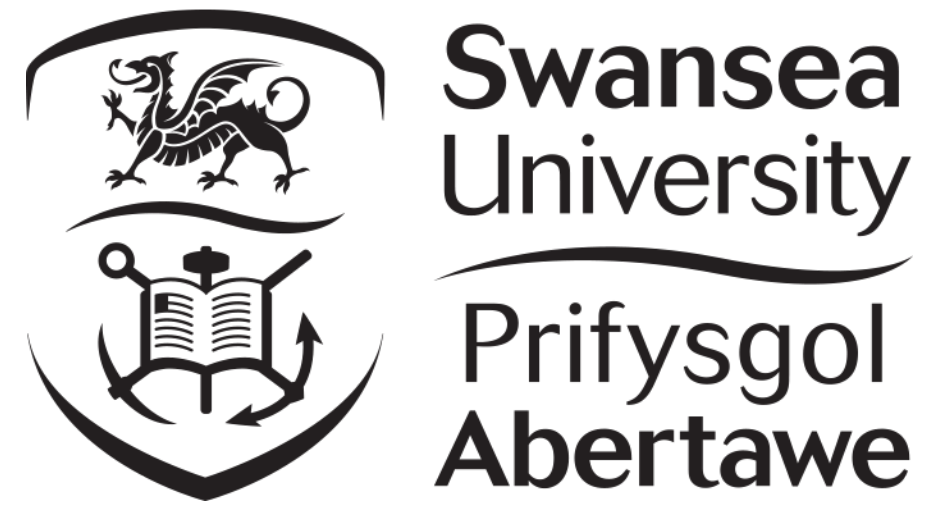

\section{Large Amplitude Oscillatory Shear (LAOS) studies of fibrin and whole blood clots}

\section{Tunde Fruzsina Lamer \\ $\mathrm{BSc}, \mathrm{MSc}$}

Submitted to Swansea University in the fulfilment of the requirements for the degree of Doctor of Philosophy 


\begin{abstract}
Venous Thromboembolism encompasses a progression of diseases where initially a blood clot is formed in the deep veins. The clot can break off, travel in the bloodstream and lodge in the pulmonary arteries leading to a lack of blood supply to the lungs. This embolic event known as pulmonary embolism can often be fatal but is difficult to predict. This thesis investigates the nonlinear rheological properties of blood clots that are important for understanding the mechanisms of embolism. The work presented uses the advanced rheological technique, Large Amplitude Oscillatory Shear (LAOS) in both strain-controlled (LAOStrain) and stresscontrolled (LAOStress) modes in order to characterise the nonlinear viscoelastic properties of fibrin and whole blood clots. Furthermore, the role of clot microstructure in the nonlinear viscoelastic properties of the mature clot was investigated. Alternating application of LAOS and Small Amplitude Oscillatory Shear (SAOS) provided detailed information of reversible and irreversible structural changes of the fibrin network revealing several distinct regions of nonlinear viscoelastic behaviour up to the point of network fracture. Shifting of these regions to different levels of stress was seen in fibrin and whole blood clots formed with different microstructures as manipulated by the addition of thrombin or heparin. Of significance, the rheologically derived value of fractal dimension, obtained from the measurement of a Gel Point using SAOS, correlated with the eventual fracture stress in a LAOStress measurement. This work suggests that incipient clot microstructure influences the ability of the fully formed clot to resist fracture as a consequence of haemodynamic forces encountered in blood vessels and sheds light on the mechanisms of changes in the conformation of the fibrin network up to the point of fracture. A main conclusion of this thesis is that the measurement of a fractal dimension of a blood clot may serve as a much needed biomarker for predicting embolism in patients whom experience thrombosis.
\end{abstract}




\section{Declaration and Statements}

\section{Declaration}

This work has not previously been accepted in substance for any degree and is not being concurrently submitted in candidature for any degree.

Signed Date

\section{Statement 1}

This work is the result of my own independent study/investigation, except where otherwise stated. Other sources are acknowledged by footnotes giving explicit references. A bibliography is appended.

Signed Date

\section{Statement 2}

I hereby give my consent for my work, if relevant and accepted, to be available for photocopying and for inter-library loan, and for the title and summary to be made available to outside organisations.

Signed

Date 


\section{Certificate of Originality}

This thesis is submitted to Swansea University under the supervision of Dr. Karl Hawkins in the Medical School in Swansea University in candidature for the degree of Doctor of Philosophy. The material in this thesis is the original work of the author except where acknowledgement to other authors is expressed.

Signed

Dated

Tunde Fruzsina Lamer

(Candidate)

Signed

Dated

Dr. Karl Hawkins

(Supervisor) 


\section{Table of Contents}

ABSTRACT

DECLARATION AND STATEMENTS........................................................................

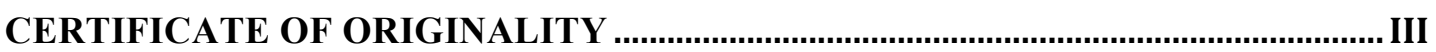

DEDICATION

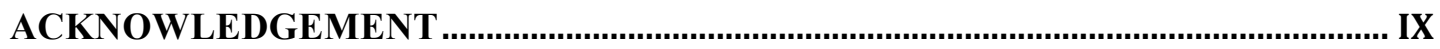

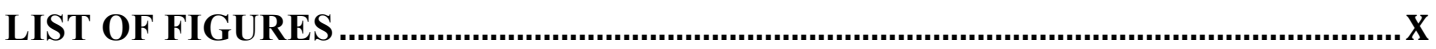

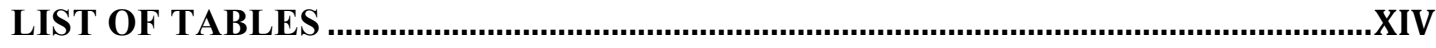

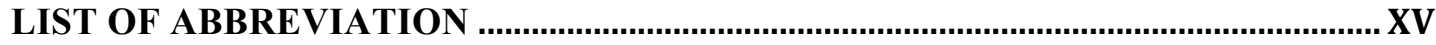

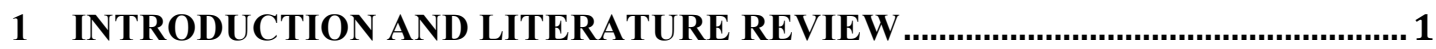

1.1 CURRENT METHODS FOR DETECTING AND TREATING DVT AND PE................................ 2

1.2 BLOOD COAGULATION ……………………………………………………………………… 7

1.2.1 Blood clot formation and the coagulation cascade .................................................. 8

1.2.2 Fibrin polymerisation................................................................................................... 11

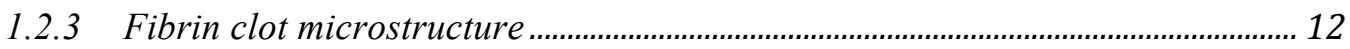

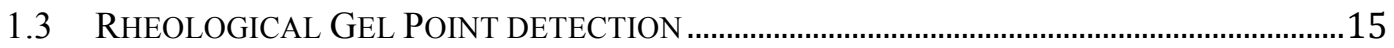

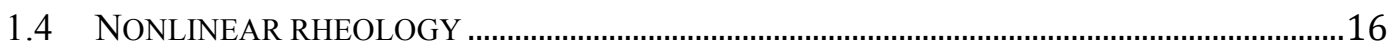

1.5 NONLINEAR RHEOLOGY OF FIBRIN ……………..........................................................

1.5.1 Nonlinear characteristics of fibrin …….............................................................. 18

1.5.2 Review of testing methods for characterising fibrin nonlinearities ..................... 19

1.5.3 Models to describe the nonlinear properties of fibrin gels.................................... 24

1.6 CLOT MICROSTRUCTURE AS A POTENTIAL BIOMARKER FOR EMBOLISM .......................25

2 THEORY

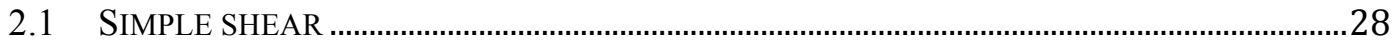

2.2 LINEAR VISCOELASTIC RANGE AND SAOS .......................................................................

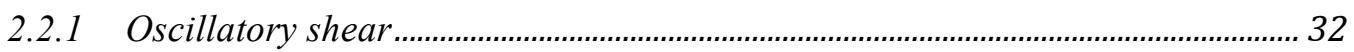

2.3 MECHANICAL MODELS OF VISCOELASTICITY ………......................................................36

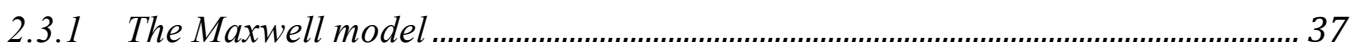

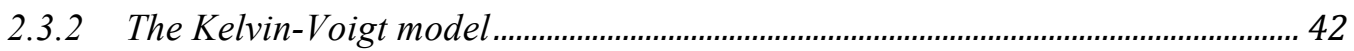

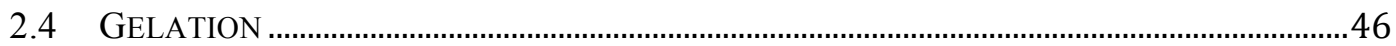

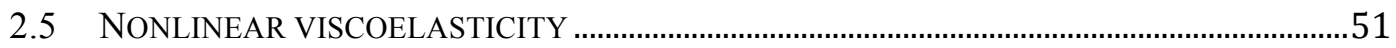

2.5.1 FT rheology

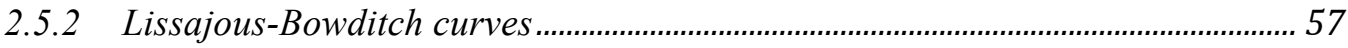




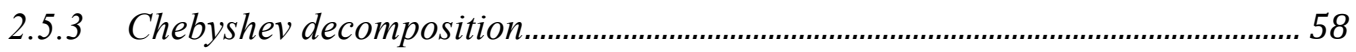

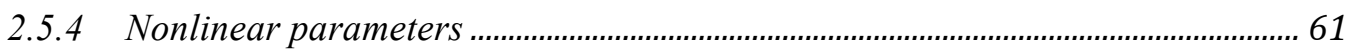

2.5.5 Stress-controlled experiments: LAOStress........................................................... 65

3 METHODOLOGY - EXPERIMENTAL APPARATUS …........................................ 71

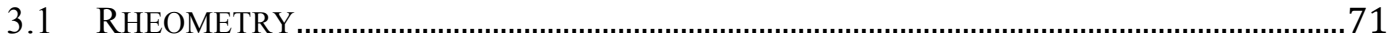

3.1.1 ARES-G2 controlled strain rheometer ……........................................................... 71

3.1.2 AR-G2 controlled stress rheometer....................................................................... 73

3.1.3 Rheometer geometries.......................................................................................... 75

3.1.4 Experimental considerations and limitations in rheometry.................................. 81

3.2 SCANNING ELECTRON MiCROSCOPE (SEM) ...................................................................85

4 LAOSTRAIN CHARACTERISATION OF FIBRIN GELS ................................... 87

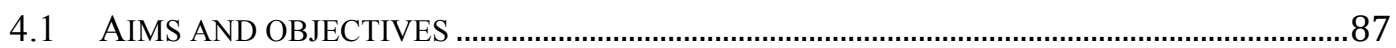

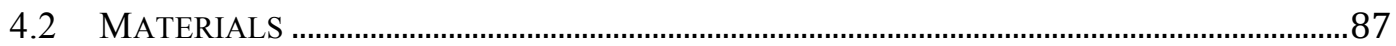

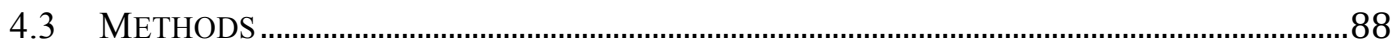

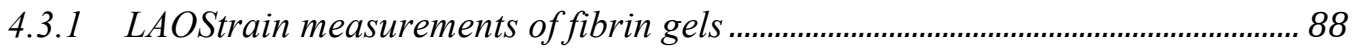

4.3.2 Effect of strain history on the fracture properties of fibrin gels........................... 89

4.3.3 Structural changes during LAOStrain ……….......................................................... 90

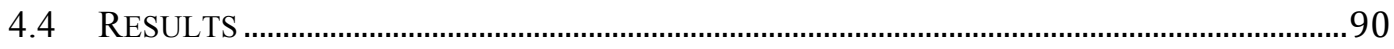

4.4.1 Steady state criteria in LAOS .................................................................................... 90

4.4.2 LAOStrain measurement of fibrin gels .................................................................... 95

4.4.3 Effect of strain history on the fracture properties of fibrin gels........................... 99

4.4.4 Structural changes during LAOS....................................................................... 101

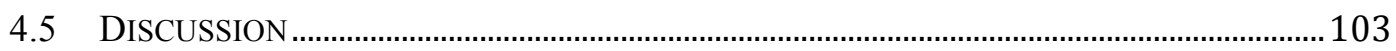

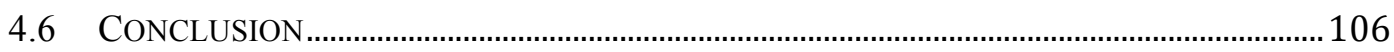

5 LAOSTRESS CHARACTERISATION OF FIBRIN GELS .................................107

5.1 AIMS AND OBJECTIVES ...................................................................................................... 107

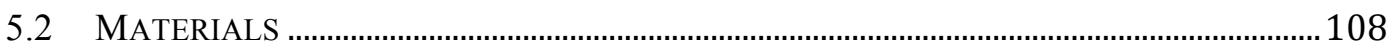

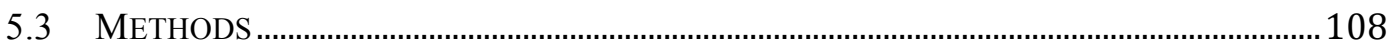

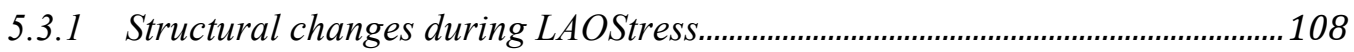

5.3.2 Fibrin clots formed under Controlled Stress Parallel Superposition.................109

5.3.3 SEM imaging of fibrin clots ................................................................................111

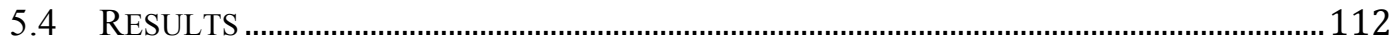

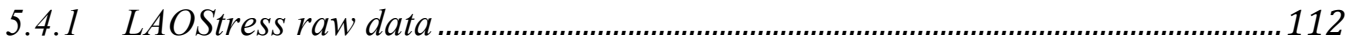

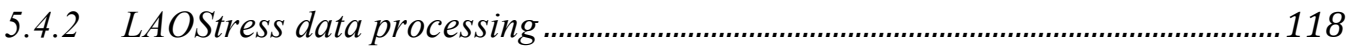

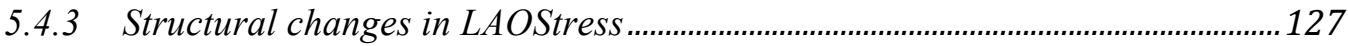


5.4.4 SEM imaging of fibrin clots probed in LAOStress measurements

5.4.5 Structural changes during LAOStress in clots formed with different thrombin concentrations

5.4.6 Effect of thrombin concentration of the fracture stress of fully formed fibrin clots 135

5.4.7 LAOStress properties of fibrin clots formed under CSPS...................................136

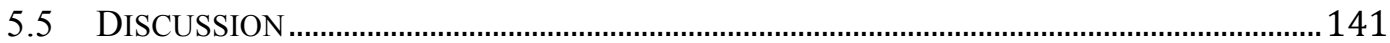

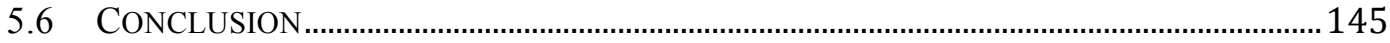

6 LAOS CHARACTERISATION OF WHOLE BLOOD ..........................................147

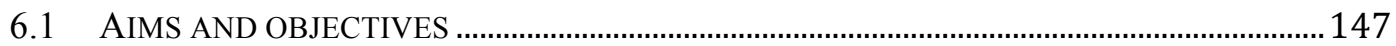

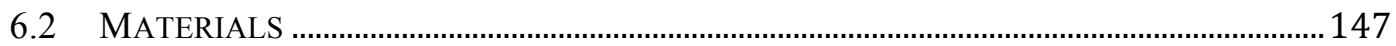

6.2.1 Whole blood clots..................................................................................................... 147

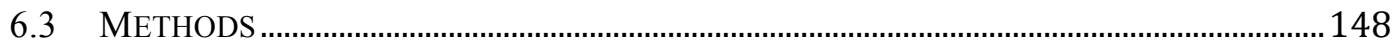

6.3.1 Gel Point measurements.....................................................................................148

6.3.2 LAOStrain of whole blood clots ........................................................................149

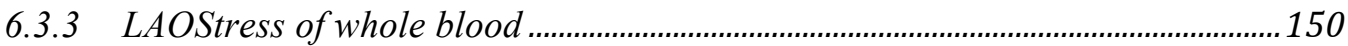

6.3.4 SEM imaging of whole blood clots......................................................................150

6.3.5 LAOSress of whole blood clots formed under CSPS...........................................151

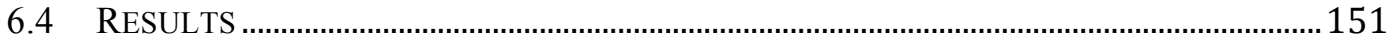

6.4.1 LAOStrain of whole blood clots .............................................................................151

6.4.2 LAOStress of whole blood clots: Lissajous-Bowditch curves...............................153

6.4.3 LAOStress of whole blood clots: probing structural changes .............................153

6.4.4 LAOStress of whole blood clots: Effects of clot microstructure..........................156

6.4.5 SEM imaging of whole blood clots.........................................................................160

6.4.6 Whole blood clots formed under CSPS..................................................................161

6.4.7 Relationship between fractal dimension and the fracture stress ........................166

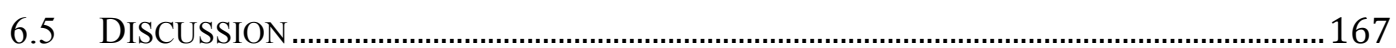

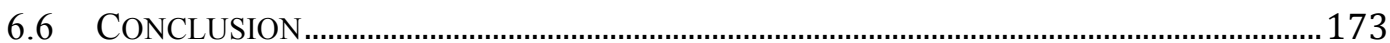

7 CONCLUSIONS AND RECOMMENDATIONS FOR FURTHER WORK …......174

8 APPENDICES

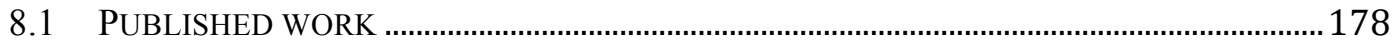

8.2 INERTIA NUMBER CALCULATION …………….......................................................... 186

8.3 EXAMPLES OF GEL POINT MEASUREMENTS ……………………………….................188

8.3.1 Gel Point measured under the application of Small Amplitude Oscillatory Shear (SAOS) 188 
8.3.2 Gel Point measured under the application of Controlled Stress Parallel Superposition (CSPS)......................................................................................................188

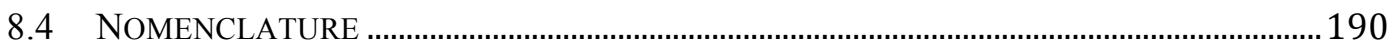

9 REFERENCES 


\section{Dedication}

I would like to dedicate this thesis to my amazing family.

Without whom none of my success would be possible. 


\section{Acknowledgement}

My sincere thanks go to my PhD supervisor, Dr. Karl Hawkins for his guidance, supervision and patience over the course of my $\mathrm{PhD}$.

I would also like to say thank you to Dr. Bethan Thomas, Dr. Nafiseh Badiei and Dr. Daniel Curtis for their invaluable support and assistance. And a special thank you for everyone who generously donated blood for my $\mathrm{PhD}$ work. The BSR and NISCHR must be thanked too for their financial support.

I would also like to thank all my friends for their support and encouragement. Last, but not least I would like to say thank you for my family for their love, endless support, patience and continuous encouragement. 


\section{List of figures}

$\begin{array}{ll}\text { Figure 1.1 Schematic representation of the coagulation cascade } & 10\end{array}$

$\begin{array}{lr}\text { Figure 1.2 Schematic representation of fibrin polymerisation } & 12\end{array}$

Figure 2.1 Schematic representation of (a) shear flow, in which the elements of the liquid are moving over or past each other, and (b) extensional flow, in which the elements of the liquid are moving towards or away from each other. Dashed line and arrows indicate the original shape of the material.

Figure 2.2 Graphical illustration of flow between parallel plates, in order to explain shear stress, shear strain and shear rate.

Figure 2.3 The schematic representation of a controlled strain rheometer in oscillatory mode

Figure 2.4 The applied sinusoidal varying strain and the resulting sinusoidal varying stress waveforms within the linear viscoelastic range

Figure 2.5 The Maxwell model

Figure 2.6 The normalised storage modulus as a function of dimensionless frequency

Figure 2.7 The phase angle as a function of dimensionless frequency

Figure 2.8 The Kelvin-Voigt model

Figure 2.9 The normalised storage and loss moduli as a function of dimensionless frequency

Figure 2.10 The phase angle as a function of dimensionless frequency

Figure 2.13 Graphical representation of the Fourier transformation (Wilhelm 2002). Time domain dataset is collected over the acquisition time, $t_{a q}$, with a certain dwell time, $t_{d w}$. The Fourier transformation of this time dependent data results in a specific frequency spectra, with spectra width of $v_{\max }$. The data acquisition time sets the minimum resolution, $\Delta v$, of the spectrum.

Figure 2.14 Example for FT-spectra of a fibrin clot that is tested within the nonlinear region

Figure 2.15 Elastic Lissajous-Bowditch plot of a fully formed, mature fibrin clot at $60 \%$ applied strain.

Figure 2.16 Graphical representation of the large-strain modulus and minimum-strain modulus

Figure 2.17 Graphical representation of the large stress elastic compliance and zero stress elastic compliance

Figure 3.1 Picture of the ARES-G2 rheometer. Arrows indicate the various components.

Figure 3.2 Schematic representation of the cross section of the AR-G2 rheometer. Grey colours indicate the stationary elements of the rheometer.

Figure 3.3 The parallel plate measuring system for (a) controlled strain rheometer and (b) controlled stress rheometer

Figure 3.4 The cone and plate measuring system for (a) strain control rheometer and (b) stress control rheometer

Figure 3.5 Schematic representation of the cross-section of a narrow gap double wall concentric cylinder geometry

Figure 3.6 Examples for incorrect and correct sample loading

Figure 4.1 Schematic representation of the procedure for measuring the effect of strain history on the fracture properties of fibrin gels. Stage 1: Application of SAOS for monitoring the microstructural evolution of the clot. Stage 2: A sinusoidal waveform with constant strain amplitude in the range of $1 \%$ to $120 \%$ is applied to the fully formed clot. Stage 3: Strain sweep between $0.1 \%$ to $300 \%$.

Figure 4.2 (a) Measured stress waveform as a function of time, and (b) absolute value of the stress waveform.

Figure 4.3 Standard deviations calculated for oscillatory cycles as a function of time.

Figure 4.4 The measured stress amplitudes as a function of time in the case of various applied strain levels from $1 \%$ to $100 \%$.

Figure 4.5 Stress difference between consecutive stress amplitudes as a function of time. 93

Figure 4.6 Stress difference between consecutive stress amplitudes in terms of percentages. $\quad 94$

Figure 4.7 Lissajous-Bowditch plots (the total stress and the corresponding elastic stress contribution as a function of strain at different strain amplitudes) of a fibrin gel formed from $4 \mathrm{mg} / \mathrm{ml}$ fibrinogen and $0.1 \mathrm{NIH} / \mathrm{ml}$ thrombin. As the strain amplitude is increasing (from (a) to (e)), the enclosed area within the loop (represents the viscous energy dissipated within a cycle (Ewoldt et al. 2010; Sun et al. 2015)) is increasing, and the clot is becoming more viscous. The maximum stress values increases until the point of fracture (d) and then decreases (e). Arrows indicate the 
stress and strain values at the point of fracture, fracture stress $\left(\sigma_{f}\right)$ and fracture strain $\left(\gamma_{f}\right)$ respectively.

Figure 4.8 Fracture stress as a function of thrombin concentration in case of crosslinked and uncrosslinked fibrin clots

Figure 4.9 Effect of the maximum complex modulus measured in the SAOS step, on the fracture stress of the fully formed fibrin clots

Figure 4.10 Frequency sweeps of the fully formed clot before the LAOS step, and after the fracture of the clot by LAOS

Figure 4.11 In a 'Strain Sweep' experiment the LVR (below 1.6 \% strain) and nonlinear region were identified (a). The fracture stress, which was measured in Stage 3 is plotted as a function of the applied constant strain amplitude in Stage 2 (b).

Figure 4.12 The measured stress responses for the applied constant strain amplitudes in Stage 2 as a function of time.

Figure 4.13 The (a) applied strain amplitudes and the response of the mature fibrin clot formed by the addition of different thrombin concentrations in terms of (b) stress, (c) $G_{L}^{\prime}$ and $G_{M}^{\prime}$ and (d) $G^{\prime}$ as a function of time.

Figure 5.1 Graphical representation of CSPS (Badiei et al. 2015). The method combines unidirectional and oscillatory shear stresses, in which the directions of the resulting shear motions are parallel to each other. In oscillatory measurements the accumulated strain is zero, whilst in CSPS it is gradually increasing.

Figure 5.2 Sample stress (upper) and strain (lower) response waveforms obtained from the rheometer software (Trios) at input stress levels of (a) $100 \mathrm{~Pa}$, (b) $500 \mathrm{~Pa}$, and (c) $1500 \mathrm{~Pa}$. These waveforms consist of discrete data points at a sampling rate of $978 \mathrm{~Hz}$.

Figure 5.3 Inertio-elastic ringing observed in a mature fibrin clot (a) in creep and (b) in LAOStress.

Figure 5.4 Results of the inertio-elastic ringing analysis: (a) inertio-elastic ringing frequency, and (b) the logarithmic decrement are plotted as a function of applied stress in creep and in LAOStress.

Figure 5.5 K' and $\mathrm{K}^{\prime \prime}$ calculated using the inertio-elastic ringing analysis in creep and in LAOStress as a function of applied stress levels.

Figure 5.6 Example of a Lissajous-Bowditch plot constructed using the raw stress and strain waveforms for input stress level of $1500 \mathrm{~Pa}$.

Figure 5.7 Smoothed sample stress (upper) and strain (lower) response waveforms obtained from the rheometer software (Trios) at input stress levels of (a) $100 \mathrm{~Pa}$, (b) $500 \mathrm{~Pa}$, and (c) $1500 \mathrm{~Pa}$. These waveforms consist of discrete data points at a sampling rate of $978 \mathrm{~Hz}$.

Figure 5.8 An example of the trimmed strain waveform that contains 15,000 data points, which is equivalent to 15 complete cycles.

Figure 5.9 Example of a strain waveform that is windowed using Hanning window function.

Figure 5.10 Example of a strain waveform that is windowed using Hanning window function and zero padded with 5000 data points.

Figure 5.11 Comparison of frequency spectra obtained from discrete Fourier transforms of the original (blue solid line) and smoothed (red dotted line) datasets for the sample stress waveform (upper) and strain response (lower) waveforms at input stress levels of (a) $100 \mathrm{~Pa}$, (b) $500 \mathrm{~Pa}$, and (c) $1500 \mathrm{~Pa}$. The magnitudes of the spectra $\left(\mathrm{I}_{\mathrm{n}}\right)$ are normalised to the magnitude of the first, fundamental harmonic response $\left(\mathrm{I}_{1}\right)$. The fundamental frequency is $1 \mathrm{~Hz}$.

Figure 5.12 Comparison of Lissajous-Bowditch curves using original (blue solid line) and smoothed (red dotted line) datasets for input stress levels of (a) $100 \mathrm{~Pa}$, (b) $500 \mathrm{~Pa}$, and (c) $1500 \mathrm{~Pa} .126$

Figure 5.13 Result of alternating SAOS and LAOStress measurements on a clot formed by the addition of $4 \mathrm{mg} / \mathrm{ml}$ fibrinogen and $0.1 \mathrm{NIH} / \mathrm{ml}$ thrombin; (a) the applied stress versus time profile, (b) the resulting strain response versus time profile, (c) large-stress and minimum-stress elastic compliances (as measured by LAOStress), and (d) linear elastic compliance (as measured by SAOS). The results reveal several nonlinear viscoelastic regions (numbered 1-4, as illustrated by the dashed lines).

Figure 5.14 SEM images of fibrin clot structures in the established viscoelastic regions.

Figure 5.15 Result of alternating SAOS and LAOStress measurements on a clot formed by the addition of $4 \mathrm{mg} / \mathrm{ml}$ fibrinogen and $0.06 \mathrm{NIH} / \mathrm{ml}$ thrombin; (a) the applied stress versus time profile, (b) the resulting strain response versus time profile, (c) large-stress and minimum-stress elastic compliances (as measured by LAOStress), and (d) linear elastic compliance (as measured by SAOS). The results reveal several nonlinear viscoelastic regions (numbered 1-4, as illustrated by the dashed lines). 
Figure 5.16 Result of alternating SAOS and LAOStress measurements on a clot formed by the addition of $4 \mathrm{mg} / \mathrm{ml}$ fibrinogen and $0.02 \mathrm{NIH} / \mathrm{ml}$ thrombin; (a) the applied stress versus time profile, (b) the resulting strain response versus time profile, (c) large-stress and minimum-stress elastic compliances (as measured by LAOStress), and (d) linear elastic compliance (as measured by SAOS). The results reveal several nonlinear viscoelastic regions (numbered 1-4, as illustrated by the dashed lines).

Figure 5.17 Nonlinear regions observed for fibrin clots formed with different thrombin concentrations in terms of applied oscillatory stress levels.

Figure 5.18 Nonlinear regions observed for fibrin clots with different thrombin concentrations in terms of resultant oscillatory strain levels.

Figure 5.19 The measured fracture stress as a function of thrombin concentration.

Figure 5.20 Result of alternating SAOS and LAOStress measurements on a clot formed under CSPS $(\sigma \mathrm{s}=0.005 \mathrm{~Pa}$ ) by the addition of $4 \mathrm{mg} / \mathrm{ml}$ fibrinogen and $0.1 \mathrm{NIH} / \mathrm{ml}$ thrombin; (a) the applied stress versus time profile, (b) the resulting strain response versus time profile, (c) large-stress and minimum-stress elastic compliances (as measured by LAOStress), and (d) linear elastic compliance (as measured by SAOS). The results reveal several nonlinear viscoelastic regions (numbered 1-4, as illustrated by the dashed lines).

Figure 5.21 Result of alternating SAOS and LAOStress measurements on a clot formed under CSPS $(\sigma \mathrm{s}=0.01 \mathrm{~Pa}$ ) by the addition of $4 \mathrm{mg} / \mathrm{ml}$ fibrinogen and $0.1 \mathrm{NIH} / \mathrm{ml}$ thrombin; (a) the applied stress versus time profile, (b) the resulting strain response versus time profile, (c) large-stress and minimum-stress elastic compliances (as measured by LAOStress), and (d) linear elastic compliance (as measured by SAOS). The results reveal several nonlinear viscoelastic regions (numbered 1-4, as illustrated by the dashed lines).

Figure 5.22 Nonlinear regions - in terms of oscillatory stress ranges - observed for fibrin clots subjected to different levels of unidirectional stress.

Figure 5.23 Nonlinear regions - in terms of oscillatory stress ranges - observed for fibrin clots subjected to different levels of unidirectional stress.

Figure 6.1 Fracture stress of whole blood clots measured as a function of fractal dimension in LAOStrain measurements.

Figure 6.2 Comparison of Lissajous-Bowditch curves of whole blood clots using original (blue solid line) and smoothed (red dashed line) datasets for input stress levels of (a) $40 \mathrm{~Pa}$, (b) $80 \mathrm{~Pa}$, and (c) $200 \mathrm{~Pa}$.

Figure 6.3 Result of alternating SAOS and LAOStress measurements on a whole blood clot formed with fractal dimension of 1.61 ; (a) the applied stress versus time profile, (b) the resulting strain response versus time profile, (c) large-stress and minimum-stress elastic compliances (as measured by LAOStress), and (d) linear elastic compliance (as measured by SAOS). The results reveal several nonlinear viscoelastic regions (numbered 1-4, as illustrated by the dashed lines).

Figure 6.4 Result of alternating SAOS and LAOStress measurements on a whole blood clot formed with the addition of $0.025 \mathrm{NIH} / \mathrm{ml}$ thrombin $\left(d_{f}=1.72\right)$; (a) the applied stress versus time profile, (b) the resulting strain response versus time profile, (c) large-stress and minimum-stress elastic compliances (as measured by LAOStress), and (d) linear elastic compliance (as measured by SAOS). The results reveal several nonlinear viscoelastic regions (numbered 1-4, as illustrated by the dashed lines).

Figure 6.5 Result of alternating SAOS and LAOStress measurements on a whole blood clot formed with the addition of $0.2 \mathrm{U} / \mathrm{ml}$ heparin $\left(d_{f}=1.45\right)$; (a) the applied stress versus time profile, (b) the resulting strain response versus time profile, (c) large-stress and minimum-stress elastic compliances (as measured by LAOStress), and (d) linear elastic compliance (as measured by SAOS). The results reveal several nonlinear viscoelastic regions (numbered 1-4, as illustrated by the dashed lines).

Figure 6.6 The effect of fractal dimension on the nonlinear viscoelastic regions measured for whole blood clots in terms on oscillatory stress

Figure 6.7 The effect of fractal dimension on the nonlinear viscoelastic regions measured for whole blood clots in terms of oscillatory strain values

Figure 6.8 SEM images of whole blood clots, and whole blood clots in the presence of $0.025 \mathrm{NIH} / \mathrm{ml}$ thrombin or $0.2 \mathrm{U} / \mathrm{ml}$ heparin

Figure 6.9 Result of alternating SAOS and LAOStress measurements on a whole blood clot formed under the application of CSPS with a higher fractal dimension value $\left(d_{f}=2.05\right)$; (a) the applied stress versus time profile, (b) the resulting strain response versus time profile, (c) large-stress and minimum-stress elastic compliances (as measured by LAOStress), and (d) linear elastic 
compliance (as measured by SAOS). The results reveal several nonlinear viscoelastic regions (numbered 1-4, as illustrated by the dashed lines).

Figure 6.10 Result of alternating SAOS and LAOStress measurements on a whole blood clot formed under the application of CSPS with a lower fractal dimension value $\left(d_{f}=1.85\right)$; (a) the applied stress versus time profile, (b) the resulting strain response versus time profile, (c) large-stress and minimum-stress elastic compliances (as measured by LAOStress), and (d) linear elastic compliance (as measured by SAOS). The results reveal several nonlinear viscoelastic regions (numbered 1-4, as illustrated by the dashed lines).

Figure 6.11 Nonlinear viscoelastic ranges in oscillatory stress levels as a function of discrete values of fractal dimension

Figure 6.12 Nonlinear viscoelastic ranges in oscillatory strain levels as a function of discrete values of fractal dimension

Figure 6.13 Measured fracture stress values of whole blood clots as a function of fractal dimension

Figure 8.1 Material's response in terms of phase angle as a function of time in Gel Point measurement under the application of SAOS

Figure 8.2 Material's response in terms of phase angle as a function of time in Gel Point measurement under the application of CSPS

Figure 8.3 Material's response in terms of shear rate as a function of time in Gel Point measurement under the application of CSPS 


\section{List of tables}

Table 2.1 Trigonometric functions and their Fourier transforms .........................................................34

Table 4.1 Components of the fibrin gel ........................................................................................8

Table 5.1 Total harmonic distortion calculated for stress and strain signals...................................... 125

Table 5.2 Summary of the different characteristics of each nonlinear region in terms of changes in linear and nonlinear compliances. $\uparrow$ represents increase, $\downarrow$ represents decrease, whilst $=$ represents unchanging values.

Table 6.2 Summary of the different characteristics of each nonlinear region in terms of linear and nonlinear compliances. $\uparrow$ represents increase, $\downarrow$ represents decrease, whilst $=$ represents unchanging values.

Table 8.1 Calculation of inertia number $\left(I_{n}\right)$ for applied stress amplitudes of $100 \mathrm{~Pa}, 500 \mathrm{~Pa}$ and 1500 


\section{List of Abbreviation}

\begin{tabular}{|c|c|}
\hline $\mathrm{ADC}$ & Analog-to-Digital \\
\hline AFM & Atomic Force Microscope \\
\hline BSP & Boltzmann Superposition Principle \\
\hline CMT & Combined Motor and Transducer \\
\hline CSPS & Controlled Stress Parallel Superposition \\
\hline $\mathrm{CT}$ & Computed Tomography \\
\hline DVT & Deep Vein Thrombosis \\
\hline ECG & Echocardiography \\
\hline FRT & Force Rebalance Transducer \\
\hline FT & Fourier Transformation \\
\hline FVL & Factor V Leiden \\
\hline GP & Gel Point \\
\hline HIT & Heparin Induced Thrombocytopenia \\
\hline HMDS & Hexamethyldisilazane \\
\hline INR & International Normalised Ratio \\
\hline LAOS & Large Amplitude Oscillatory Shear \\
\hline LAOStrain & Large Amplitude Oscillatory Strain \\
\hline LAOStress & Large Amplitude Oscillatory Stress \\
\hline LMHW & Low-Molecular Weight Heparin \\
\hline LVR & Linear Viscoelastic Region \\
\hline MAOS & Medium Amplitude Oscillatory Shear \\
\hline MRV & Magnetic Resonance Venography \\
\hline $\mathrm{NIH}$ & National Institute of Health \\
\hline PBS & Phosphate Buffered Saline \\
\hline $\mathrm{PE}$ & Pulmonary Embolism \\
\hline PESI & Pulmonary Emboli Severity Index \\
\hline PPP & Platelet Poor Plasma \\
\hline PRP & Platelet Rich Plasma \\
\hline $\mathrm{RBC}$ & Red Blood Cell \\
\hline SAOS & Small Amplitude Oscillatory Shear \\
\hline SEM & Scanning Electron Microscope \\
\hline
\end{tabular}




$\begin{array}{ll}\text { SMT } & \text { Separate Motor and Transducer } \\ \text { s-PESI } & \text { Simplified Pulmonary Emboli Severity Index } \\ \text { TBS } & \text { Tris-Buffered Saline } \\ \text { TF } & \text { Tissue Factor } \\ \text { THD } & \text { Total Harmonic Distortion } \\ \text { UFH } & \text { Unfractionated Heparin } \\ \text { VEL } & \text { Viscoelastic Liquid } \\ \text { VES } & \text { Viscoelastic Solid } \\ \text { vWf } & \text { von Willebrand factor } \\ \text { VTE } & \text { Venous Thromboembolism } \\ \text { WB } & \text { Whole Blood } \\ \text { WHO } & \text { World Health Organisation }\end{array}$




\section{Introduction and Literature review}

Venous Thromboembolism (VTE) is one of the leading causes of death and disability. Each year, 10 million cases of VTE are recorded worldwide (Jha et al. 2013). In the United Kingdom, more than one in 1000 people is affected by Deep Vein Thrombosis (DVT), whilst 86 in 100,000 people are affected by Pulmonary Embolism (PE) annually (Bayer HealthCare 2013). Moreover, VTE causes more than 25,000 potentially preventable deaths per annum in UK hospitals (Report to the Chief Medical Officer 2007). The Welsh government has reported that $70 \%$ of deaths caused by blood clots acquired in hospitals in Wales could have been prevented with appropriate preventative measures (National Assembly for Wales Health and Social Care Committee 2012). For these reasons, early intervention and prevention of thrombosis and its associated complications are crucial. In addition to the disease burden, VTE causes serious economic burden. Yearly, VTE costs $£ 640$ million for the National Health Service in the UK (Worldthrombosisday.org 2018). It is undeniable that many people are affected by this potentially life threatening disease, and it also creates significant economic burden worldwide.

The main purpose of this project is to investigate a structural biomarker of blood clots, which might predict the risk of embolism in patients diagnosed with DVT. DVT is a disease in which case a blood clot is formed in the deep veins - most often in the leg - and partially or fully blocks the blood vessels. As a complication of DVT, the formed blood clot can break off, travel in the blood stream and lodge in the pulmonary arteries leading to a lack of blood supply to the lungs. This embolic event is called PE. The size of the emboli in an event of PE can vary, it can either impair or completely block the pulmonary circulation, therefore a large emboli is fatal (Martinez et al. 2014). DVT and PE are collectively termed as VTE. However, there is no reliable method available for predicting an embolic event in VTE patients, and the mechanisms behind embolism are not fully understood.

A recent study has reported abnormal clot microstructure in blood taken from VTE patients undergoing warfarin therapy (Lawrence et al. 2015). The microstructure of 
anticoagulated VTE blood, in terms of the rheometrically derived fractal dimension $\left(d_{f}\right)$, was indistinguishable from healthy, non-anticoagulated blood. Preliminary data showed differences in $d_{f}$ of patients suffering from DVT only $\left(d_{f}=1.719 \pm 0.0464\right)$ and those who experienced both DVT and PE $\left(d_{f}=1.756 \pm 0.0417\right)$. This finding suggests that the clot microstructure plays a crucial role in the etiology of embolism, and the underlying microstructure of the clot might influence the propensity of the clots to fracture. $d_{f}$ quantifies the structural complexity of the incipient clot that predicts the fully formed clot structure and could be a biomarker for embolic events.

The research herein utilises the advanced rheometrical technique, i.e. Large Amplitude Oscillatory Shear (LAOS), to study the underlying mechanism of clot fracture. As it is hypothesised that the measured nonlinear properties are related to a potential biomarker for embolism, the rheometrically derived fractal dimension, $d_{f}$, which provides information of the microstructure of the incipient clot.

\subsection{Current methods for detecting and treating DVT and PE}

Blood clots can form in the blood vessels without any previous warning signs or symptoms. DVT might cause pain or tenderness in the affected leg, swelling of calf, ankle and foot, red discolouration and warmth of the affected limb (Shakerian \& Sadeghipour 2018; Barnes et al. 2014). Symptoms for PE include shortness of breath, rapid breathing and rapid heart rate, chest pain, and light-headedness. It is important to note, that these symptoms or signs are not specific for PE; therefore it can be misdiagnosed as acute coronary syndrome, or other cardiovascular disease (Shakerian \& Sadeghipour 2018). Healthcare professionals are recommended to perform a VTE risk assessment (i.e. Wells score) on all patients before they are admitted to hospital (NICE 2012). They gather information regarding the patient's age, medical history and other lifestyle factors (e.g. obesity, pregnancy, smoking or alcohol consumption), which help to identify those at risk of developing VTE.

Strong risk factors include:

- staying in hospital bed for a long period of time,

- undergoing hip, knee or cancer related surgery, 
- $\quad$ long-haul travel ( $>4-6$ hours).

While some of the moderate risk factors are

- personal or family history of VTE,

- cancer, undergoing chemotherapy,

- using oestrogen-based medication.

The most well-known anatomic risk factor of VTE is the May-Turner syndrome, or iliac vein compression syndrome, in which condition, the left common iliac vein is subjected to compressive force generated by the right common iliac vein, which promotes clot formation (May \& Thurner 1957). Genetic risk factors of VTE include factor V Leiden (FVL) and prothrombin mutation (Shakerian \& Sadeghipour 2018). FVL is a common prothrombotic polymorphism amongst Caucasian people. In a paradoxical manner, FVL increases the risk of DVT, however individuals with FVL show less tendency for developing PE (Corral et al. 2010).

Based on the information gathered, the Wells score is calculated, and individuals are classified as at high (>3 points), moderate (1-2 points) or low $(<0)$ risk for developing blood clots in their blood vessels (Shakerian \& Sadeghipour 2018). The patients who are at risk should be given suitable prophylaxis, such as anticoagulant medication or medical devices (e.g. compression stocking, intermittent pneumatic compression devices or rapid inflation venous foot pumps) (Worldthrombosisday.org 2018). Other diagnostic tools are also applied alongside the Wells score prediction system, in order to overcome its limitations (e.g. human factor). Diagnostic methods for DVT include D-dimer tests, compression ultrasonography, magnetic resonance venography (MRV), and contrast venography (Shakerian \& Sadeghipour 2018).

The most common clinical diagnostic tool for DVT is the D-dimer test (Shakerian \& Sadeghipour 2018). D-Dimer tests detect the level of D-dimer, which is a crosslinked fibrin degradation product, within the blood stream. Elevated level of D-dimer ( $>$ $500 \mathrm{ng} / \mathrm{ml}$ ) suggests that a blood clot is present in the vein. However, the D-dimer test is not always reliable and it can give false positive results; hence as a single test, it is only useful for excluding DVT (except for individuals who are at high risk based 
on their Wells score). False positive D-dimer results can be attributed to other disease states, which also increase the D-dimer concentration, such as myocardial infarction, stroke, pregnancy etc. Therefore, in the case of a positive D-dimer test result, performing additional diagnostic methods is required (Gruber \& Bull 2012; Nhs.uk 2016).

Compression ultrasonography is a non-invasive approach, which might be used for detecting clots in the veins. This approach investigates signs of abnormal venous compressibility, and the Doppler ultrasound is used for monitoring the speed of the blood flow within the blood vessel. Abnormalities of the blood flow, i.e. slower or blocked blood flow, could indicate the presence of a blood clot. Advantages of this technique include high sensitivity and ease of use (Schellong et al. 2003; Nhs.uk 2016). However, a disadvantage of the technique is that it cannot detect clots in the iliac veins (Shakerian \& Sadeghipour 2018).

A highly specific, highly sensitive, and non-invasive diagnostic tool for DVT is magnetic resonance venography (MRV) (Shakerian \& Sadeghipour 2018). If the results from D-dimer and ultrasound tests are inconclusive, or ultrasonography cannot be performed (i.e. in iliac veins, or patients with leg deformities), MRV is used for diagnostic purposes. Despite its great advantages, MRV is an expensive test to conduct, and it requires expertise for interpreting the test results.

Contrast venography is a very effective method for DVT detecting, in which case contrast dye is injected into the vein in the foot. X-ray is used to follow the dye travelling up the leg; hence clots that are stopping the blood flow can be detected (Nhs.uk 2016). Despite its effectiveness, nowadays this method is rarely used only for diagnosing DVT, since it is an invasive method (Shakerian \& Sadeghipour 2018).

In the case of a low risk Wells score, a negative D-dimer test or negative compression ultrasound rules out the possibility of DVT. For a moderate or high risk Wells score, both the D-dimer test and the ultrasound test have to be performed, and both methods should show negative results in order to confirm that DVT is not present. Whilst individuals who show high Wells score should be tested with compression ultrasound alongside with MRV or contrast venography, and both 
testing methods should show negative results to confidently exclude DVT as diagnosis (Shakerian \& Sadeghipour 2018).

As mentioned earlier, PE might be misdiagnosed as other cardiovascular disease, as its symptoms are not specific. Similarly to DVT, Wells score is evaluated for patients in order to determine whether they are at risk of developing PE. Other prediction methods for PE include Modified Wells score, Simplified Wells score, Revised Geneva score and Simplified revised Geneva score (Wells et al. 2000; Gibson et al. 2008; Le Gal et al. 2006; Klok et al. 2008). These diagnostic prediction methods were compared and evaluated in terms of sensitivity, specificity, efficiency and failure rate in a recent study by Hendriksen et al. (Hendriksen et al. 2015). Efficiency of all methods investigated was between $43-48 \%$. Diagnostic tools for PE include D-dimer testing, electrocardiography, and imaging methods, such as echocardiography (ECG), chest cardiography, lung scanning, chest computed tomography (CT), magnetic resonance angiography and pulmonary angiography (Shakerian \& Sadeghipour 2018). ECG and chest cardiography helps to rule out other conditions, such as myocardial infarction and pneumonia, respectively. Currently, CT is considered as the most reliable tool for diagnosing PE. It is a quick and non-invasive method that uses X-rays in order to generate a cross-sectional image of the chest (Shakerian \& Sadeghipour 2018). The detected PE is classified based on its size as a high (large emboli), intermediate (sub massive emboli) or low risk PE. Another PE risk assessment tool is the Pulmonary Emboli Severity Index (PESI), and its simplified version, s-PESI (Aujesky et al. 2005; Vinson et al. 2016). PESI score is based on 11 criteria, while the s-PESI determines the severity of PE based on 6 criteria.

VTE is a potentially life-threatening disease; therefore it requires immediate medical attention. The current treatment for VTE includes the administration of anticoagulants, such as injectable heparin or low molecular weight heparin, or dabigratan, apixaban, rivaroxaban, edaxaban and warfarin tablets; thrombolytic therapy, such as tissue plasminogen activator; or invasive therapies (Shakerian \& Sadeghipour 2018). Thrombolytic therapies are not commonly applied for treatment of DVT. However, in the case of a high risk PE fibrinolytic therapy is recommended. Surgical management of VTE is also limited. Except for PE patients with a large 
emboli, in which case the surgical procedure is important, as it could be lifesaving (Shakerian \& Sadeghipour 2018).

Anticoagulant therapy is the most commonly used treatment of VTE, and it starts once the patient is identified as moderate or high at risk of developing PE (Konstantinides \& Goldhaber 2012). The purpose of the treatment is preventing further clot formation, embolisation of the existing clot, and other complications of VTE, such as post-thrombotic syndrome (PTS) (Barnes et al. 2014). Administration of warfarin, which is a vitamin $\mathrm{K}$ antagonist, is the oldest and predominant anticoagulant therapy for treating VTE (Shakerian \& Sadeghipour 2018; Konstantinides \& Goldhaber 2012). During warfarin treatment, frequent blood tests are required to monitor the International Normalised Ratio (INR) of the patients, in order to monitoring and adjusting the warfarin dosage. INR refers to standardised prothrombin time, introduced by the World Health Organisation (WHO) and the International Committee on Thrombosis and Haemostasis in order to report standardised results of blood coagulation assays. The INR number is calculated using the ratio of the patient's prothrombin time and the mean normal prothrombin time, and an International Index which is specific to each batch of thromboplastin reagent used. The prothrombin time describes the clotting tendency of blood. It is concerning that despite the continuous monitoring of INR of patients undergoing warfarin therapy, in some cases recurrent embolic event occurs even in patients whose blood is fully anticoagulated according to the INR value (Kearon et al. 2008; Thachil 2012).

The novel oral anticoagulant treatments, apixaban and rivaroxaban have less serious side effects compared to heparin or warfarin. In addition, prior to apixaban or rivaroxaban treatment parental anticoagulant therapy is not required, unlike in the case of dabigratan and edaxaban (Shakerian \& Sadeghipour 2018).

Parental anticoagulant treatments include the administration of unfractionated heparin (UFH), low-molecular weight heparin (LMWH), and fondaparinux (Shakerian \& Sadeghipour 2018). Administration of heparin, as anticoagulant medication can trigger an adverse immune response called heparin-induced thrombocytopenia (HIT) in some patients. HIT results in formation of clots 
throughout the cardiovascular system, which could cause strokes, amputation, and it can even be fatal (Franchini 2005; Thomas 2014). Treatment with LMWH and fondaparinux are more beneficial than with UFH, due to the ease of adjusting the dosage, and to the lower risk of HIT (Shakerian \& Sadeghipour 2018).

However, VTE is one of the leading causes of death and disability worldwide, it is a potentially preventable disease. Therefore, it is important to identify those at risk of VTE, and give them appropriate prophylaxis. Prophylaxis of VTE include low dosage of anticoagulant medication; or using medical devices, such as intermittent pneumatic compression and compression stockings (Shakerian \& Sadeghipour 2018).

\subsection{Blood Coagulation}

Blood is a body fluid that circulates within the body through blood vessels and transports substances such as oxygen, nutrients, hormones and carbon dioxide. It also performs immunological functions, such as circulating white blood cells. Many studies and even journals (for example Clinical Hemorheology and Microcirculation) focus on the rheological properties of blood (i.e. Haemorheology). Blood under flow is a non-Newtonian liquid, since its viscosity depends on the applied shear rate. The non-Newtonian characteristics of blood include viscoelasticity, yield stress, shear thinning, and thixotropic behaviours (Charm \& Kurland 1967; Oka 1981; Bodnár et al. 2011; Thurston 1972; Dintenfass 1962; Gijsen et al. 1999). These rheological features are mostly attributed to the red blood cells (RBCs), which are present in a large concentration in comparison to other cellular elements (Bodnár et al. 2011). The viscosity of blood decreases as the applied shear rate increases; therefore fluid blood displays shear thinning characteristics (Gijsen et al. 1999). Below a critical stress value (i.e. yield stress) blood ceases to flow. This rheological property of blood is attributed to the rouleaux formation, which is a physiological process of a special form of $\mathrm{RBC}$ aggregation under low shear rates and enhanced by increased fibrinogen concentration (Charm \& Kurland 1967; Oka 1981). Haemorheology can be potentially applied for clinical diagnostic purposes, since the viscoelastic characteristics of the blood provide biomarkers of abnormal coagulation in various 
disease (Evans et al. 2010; Lawrence et al. 2014; Lawrence et al. 2015). Previous studies have investigated the rheological properties of blood in its fluid state, under linear viscoelastic conditions (Pirofsky 1952; Kim et al. 2000; Hitosugi et al. 2001), as well as behaviour of blood flow in nonlinear viscoelastic state, by the application of Large Amplitude Oscillatory Shear (LAOS) (Sousa et al. 2013). Recent studies have focused on the complex rheological process of clot formation, in which the blood transitions from a viscoelastic liquid into a viscoelastic solid (Evans et al. 2008; Evans et al. 2010). Understanding the process of clot formation is important. Normal clot formation is a physiologically relevant process, which is triggered by vascular injury (Roberts \& White 2017). The role of the formed impermeable fibrin clot is to stop blood loss in the case of an injury. The factors present in the blood are responsible for clot formation (i.e. haemostasis), which in normal physiological conditions should be restricted to the area affected by the vascular injury. The vessel wall, blood platelets and soluble procoagulant and anticoagulant components are involved in the controlled mechanism of haemostasis. However, in disease states blood clots might form within the blood vessel, leading to partial or full occlusion. In the case of DVT the undesired clot forms in the deep veins. This clot can embolise, and the clot that breaks off can travel in the blood stream and lodge in the pulmonary arteries, causing a potentially fatal pulmonary embolism. Therefore, it is also crucial to investigate the nonlinear rheological properties of the fully formed clot, as it might allow understanding of the mechanisms behind embolism. Measuring the nonlinear behaviour of fibrin clots has been the focus of some previous research, which are further discussed in detail within Chapter 1.5. However the pathophysiology and etiology of embolism is still not fully understood, which is why further research is necessary within this field.

\subsubsection{Blood clot formation and the coagulation cascade}

Haemostasis consists of three processes; primary haemostasis, secondary haemostasis and fibrinolysis. Primary haemostasis is the early stage of clot formation, in which a primary platelet plug is formed (Clemetson 2012). In normal circumstances, the circulating platelets are separated from the procoagulant subendothelial matrix by the endothelium. Damage to the endothelium immediately 
triggers primary haemostasis, as the prothrombotic haemostatic factors (i.e. collagen, von Willebrand factor (vWf), fibronectin and tissue factor (TF)). In the first step of primary haemostasis, the platelets adhere directly or through $\mathrm{vWf}$ to the subendothelial matrix on the site of injury. The adhered platelets become activated as they adhere to the endothelial cells, and this brings more platelets to the injury site. The activated platelets provide binding sites for the coagulation factor complexes, hence promoting fibrin formation (secondary haemostasis). In the final stage of primary haemostasis, the activated platelets aggregate, and fibrinogen binds to the activated fibrinogen receptor on the surface of platelets, hence connecting the platelets and forming the platelet plug (Austin 2017).

Secondary haemostasis, or coagulation is the process of blood clot formation, in which the blood is transitioning from a liquid-like state into a solid-like state (Roberts \& White 2017). The coagulation cascade describes this process in a series of steps, in which proenzymes are proteolytically converted into enzymes by activators and cofactors. The coagulation cascade might be divided into two main pathways, extrinsic or tissue factor (TF) activated pathway, and intrinsic or contact activated pathway which join to form the common pathway (Figure 1.1).

The extrinsic (TF activated) pathway is triggered upon trauma. The blood vessel gets damaged, and as a result factor VII (FVII) leaves the circulation, comes in contact with TF and together form the activated complex, TF-FVIIa. The TF-FVIIa complex activates both factor IX (FIX) and factor X (FX).

The intrinsic (contact activated) pathway is initiated by a foreign surface, which activates factor XII (FXII or Hageman factor) into activated factor XII (FXIIa). FXIIa activates factor XI (FXI). The created FXIa coverts FIX into activated factor IX (FIXa). FIXa together with its cofactor, activated factor VIII (FVIIIa) form the "tenase" complex, which activates FX.

In the common pathway, the activated factor $\mathrm{X}(\mathrm{FXa})$ rapidly converts prothrombin (factor II, FII) into thrombin (activated factor II, FIIa). Thrombin triggers the polymerisation of fibrinogen (factor I, FI) into fibrin (activated factor I, FIa). Finally, 
activated factor XIII (FXIIIa) initiates the formation of covalent crosslinks, leading to the formation of crosslinked fibrin clot.

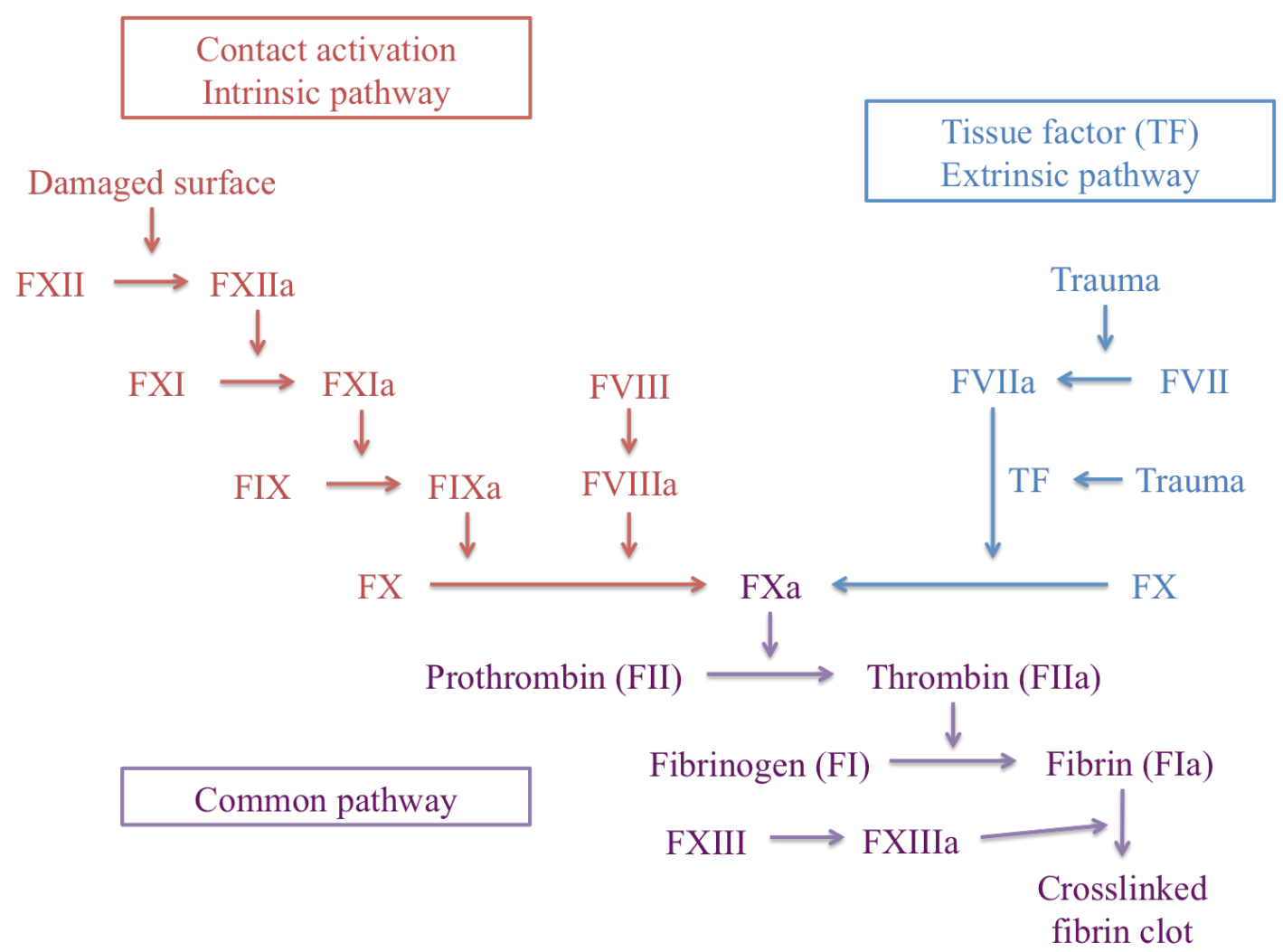

Figure 1.1 Schematic representation of the coagulation cascade

The formed blood clot consists of platelets, red blood cells (RBC), and leucocytes that are trapped within the mesh of fibrin fibres (Roberts \& White 2017; Gersh et al. 2009). The fibrin network in which the blood cells are captured is created in a polymerisation process, in which fibrinogen is converted into fibrin by the enzyme thrombin. This process is discussed more extensively in the following section (Chapter 1.2.2).

Fibrinolysis is the process in which fibrin is digested by plasmin, and leads to the dissolution of a formed clot or thrombus (Collen \& Lijnen 1991; Weisel \& Litvinov 2014). Plasmin is the central enzyme of fibrinolysis; it is a serine protease formed from its inactive precursor, plasminogen, by the action of plasminogen activators. Apart from fibrin digestion, plasmin cleaves other substances, such as extracellular matrix proteins, and activates other proteases and growth factors. Therefore, plasmin and plasminogen are also involved in cell migration, wound healing, inflammation, 
embryogenesis, ovulation, angiogenesis, tumour growth and metastasis, and atherosclerosis.

\subsubsection{Fibrin polymerisation}

Fibrin clot formation is an essential part of haemostasis, and can be triggered by vascular injury, and the resulting fibrin clot acts as a provisional extracellular matrix (Chernysh et al. 2012; Jansen et al. 2013). During the process of fibrin clot formation the soluble fibrinogen $(340 \mathrm{kDa})$ is polymerised by the enzyme thrombin to form insoluble fibrin. The precursor of fibrin, fibrinogen is a fibrous protein, which contains three pairs of polypeptide chains: $A \alpha, B \beta$ and $\gamma$ that are connected by 29 disulphide bonds. As shown in Figure 1.2, the polymerisation process starts from the centre of the fibrinogen molecule, on the cleavage of fibrinopeptides $\mathrm{A}$ and $\mathrm{B}$, as fibrin monomers are forming. The fibrin monomers polymerise into half-staggered oligomers, which are then lengthened to form protofibrils, which self assembles into thick fibrin fibres as aggregates laterally (Chernysh et al. 2012; Ferry 1952). The fibrin gel and 3D structure is created as the fibrin fibres are branching and growing longitudinally and laterally. Transglutaminase (FXIII) forms crosslinks, covalent bonds between the fibres, which stabilize the structure of the fibrin gel (Chernysh et al. 2012). The formed fibrin network shows some remarkable mechanical properties under the application of large stress or strain (Janmey et al. 1983; Bale \& Ferry 1988; Liu et al. 2010; Hudson et al. 2010; Piechocka et al. 2010; Münster et al. 2013; van Kempen et al. 2015). 

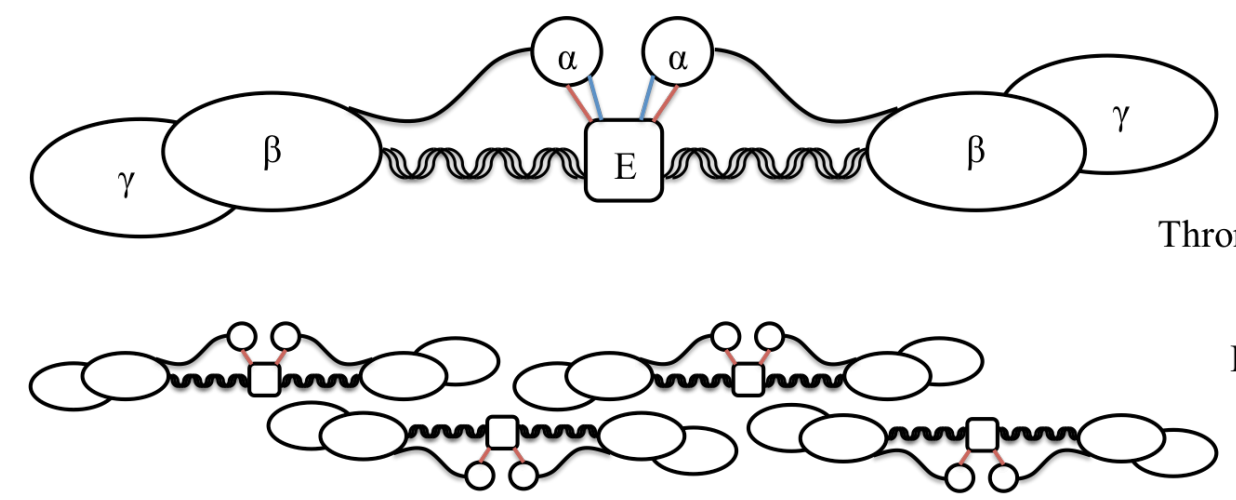

Thrombin

Fibrinogen

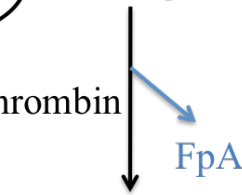

Fibrin I and protofibril formation

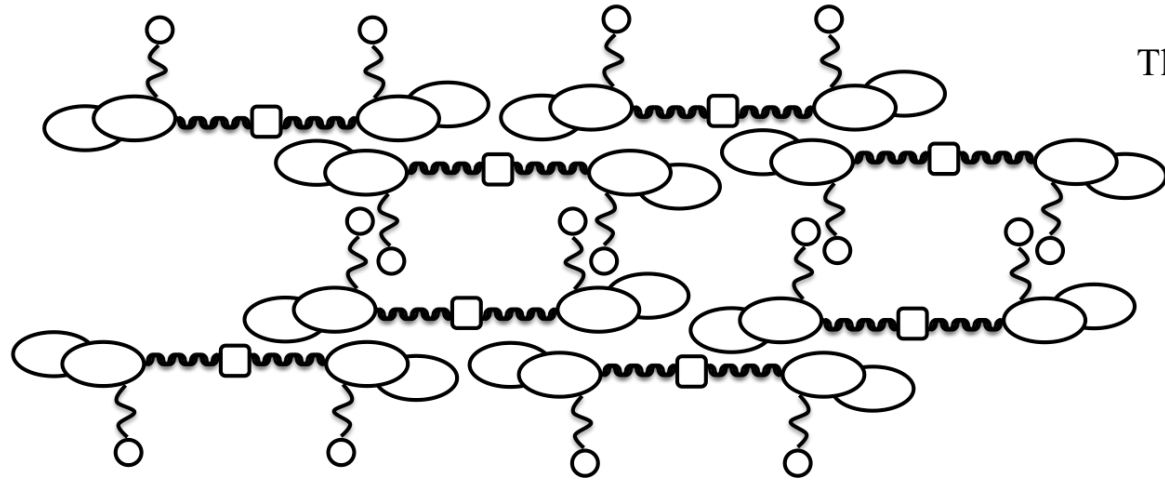

Thrombin

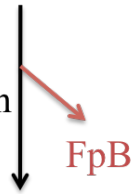

Fibrin II and lateral aggregation

Figure 1.2 Schematic representation of fibrin polymerisation

\subsubsection{Fibrin clot microstructure}

The underlying 3-dimensional microstructure composed of the clot's fibrin network is very important, because it will determine the mechanical and chemical stability of the clot (Shah \& Janmey 1997; Weisel \& Litvinov 2013b; Undas \& Ariens 2011; Gersh et al. 2009). Alterations in fibrin architecture have been linked to various diseases, such as heart attack, stroke, cancers, surgical complications, and bleeding disorders.

The formed fibrin clot structure can vary, and the factors that are causing these variations can be classified into four main groups (Weisel \& Litvinov 2013b):

- Hereditary or acquired variations in fibrin structure,

- Environmental conditions during polymerisation,

- Cells in the blood,

- Haemodynamic flow. 
Different fibrin clot structures can form due to hereditary or acquired changes in fibrinogen splice variants, single-nucleotide polymorphisms, and posttranslational alterations to the fibrinogen molecule (Weisel \& Litvinov 2013b). As a result of the variations in fibrinogen structure, the fibrin polymerisation, the stability of the formed clot and the susceptibility of formed clots to fibrinolysis can be affected (Hoffman 2008; de Moerloose \& Neerman-Arbez 2009). Fibrinogen is very prone to oxidation; therefore oxidative stress results in alterations of the fibrinogen molecule, and the forming fibrin clot (Undas \& Ariens 2011). Altered fibrinogen structure due to oxidation, has been shown to alter fibrin clot structure, in such a way that the formed clots are less permeable and more resistant to fibrinolysis (Undas et al. 2008).

The formed fibrin clot microstructure is affected by various environmental conditions during the polymerisation process; these include the ionic conditions (i.e. $\mathrm{pH}$, ionic composition and strength), endogenous and exogenous compounds (e.g. polyphosphates, peptides, lipids), medical treatments, and the presence of normal and pathological proteins (e.g. albumin, fibronectin) (Weisel \& Litvinov 2013b). The structure of the fibrin clot is significantly affected by the concentration of active thrombin. The level of active thrombin present is based on the relative ratio of enzyme generation and elimination. Fibrin clots formed in the presence of high thrombin concentration have a more dense clot structure, with lots of thin fibres and more branching points, with smaller pore space. Whilst, low thrombin concentration leads to the formation of a more porous, more open fibrin clot structure, with fewer but thicker fibrin fibres and fewer branching points (Weisel \& Litvinov 2013b). In addition, progressive haemodilution results in more open, porous fibrin clot microstructures (Lawrence et al. 2014).

Cellular elements present in the blood affect the clot structure; hence altering some of their properties, such as stiffness, clot stability and resistance to fibrinolysis (Weisel \& Litvinov 2013b; Wolberg 2012). The presence of platelets causes clot retraction (i.e. highly increased densification of the fibrin network), and surrounding platelet aggregates the fibrin network is more dense, consisted of many thin fibres, which structure is different from the bulk fibrin network (Feghhi \& Sniadecki 2011; Collet et al. 2002; Campbell et al. 2008; Lam et al. 2011). The red blood cells present 
in the clot affect the thickness of the fibres, and the mechanical properties and heterogeneity of the clot (Gersh et al. 2009). In addition, red blood cells prevent platelet binding, and therefore inhibit the level of contraction caused by platelets (Riha et al. 1999; Gersh et al. 2009).

The flow of blood also affects the formation of the fibrin network, since the fibres align in the direction of flow, and therefore the structure of the formed clot, its mechanical properties, and susceptibility to fibrinolysis are altered (Weisel \& Litvinov 2013b; Gersh et al. 2010; Campbell et al. 2010; Whittaker \& Przyklenk 2009; Varju et al. 2011). A recent study by Colace et al. applied a microfluidic approach in order to investigate clot growth (Colace et al. 2012). They showed that as the clot is growing, the shear stress caused by the blood flow increases until $80 \%$ of total vessel occlusion, followed by a rapid decrease in stress. Hence, it is important to consider the effect of increasing levels of stresses caused by the blood flow on the clot, and these factors could determine the probability of the clot to embolise.

The formed fibrin network structure is often characterised by Scanning Electron Microscopy (SEM). Figure 1.3 shows fibrin clot structures formed with a higher and with a lower thrombin concentration. SEM images can be assessed qualitatively or quantitatively. SEM image of a fibrin network can be described in qualitative terms, such as dense, less porous (Figure 1.3b), or open and porous structure (Figure 1.3a), with more/less branch points, nature of branch points and with thicker/thinner fibres (Gersh et al. 2009; Weisel \& Litvinov 2013b). Typically, fibrin fibre network with more branch points consists of thinner fibres, whilst fibrin network with less branch points contains thicker fibres. The 3-dimensional fibrin network might be assessed quantitatively, by measuring the size of the pores, branching density, distance between branch points, total number of branch points, fibre distribution, fibre length and fibre diameter (Weisel 2007; Weisel \& Litvinov 2013b). 
a)

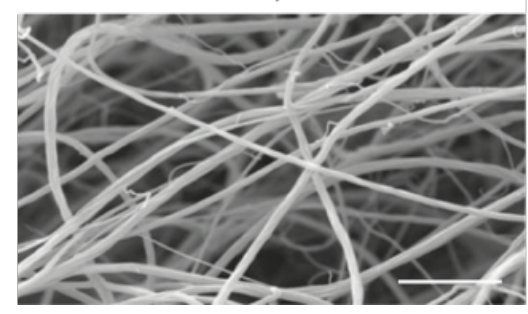

b)

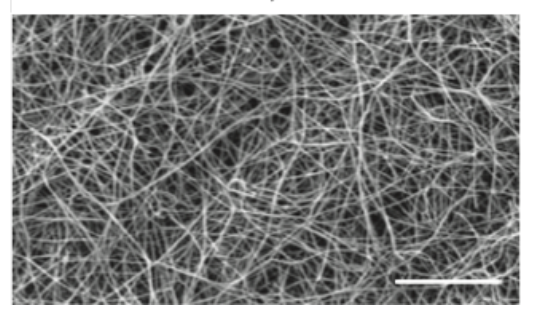

Figure 1.3 Scanning electron micrographs of plasma clots formed in the presence of a) low thrombin concentration and b) high thrombin concentration. Magnification bar $=5 \mu \mathrm{m}$ (Weisel 2007)

\subsection{Rheological Gel Point detection}

Fibrin or blood clot formation results from gelation and undergoes a liquid to solid transition. During this transition, the material undergoes a critical point, its Gel Point (GP), which is the point when the 3-dimensional sample spanning network of branching fibres is established (Winter 2002). The process of clotting can be monitored, and the GP can be detected in a rheological measurement by the application of Small Amplitude Oscillatory Shear (SAOS) (Evans et al. 2010; Hawkins et al. 2010). During the GP measurement the material is subjected to a sinusoidal stress or strain waveform at different frequencies, within the linear viscoelastic region (LVR), so that the level of applied stress or strain does not disrupt the microstructure of the material. From the values of viscoelastic properties at the GP, the fractal dimension $\left(d_{f}\right)$ can be calculated which serves as a structural biomarker of coagulation (Lawrence et al. 2015; Evans et al. 2010; Lawrence et al. 2014). (Terms such as SAOS, LVR, GP measurement and $d_{f}$ introduced herein are further discussed in Chapter 2)

The fractal dimension provides meaningful information about the microstructure of the incipient clot. The fractal dimension of healthy whole blood has been identified as $d_{f}=1.74( \pm 0.07)$ (Evans et al. 2010). Anticoagulant medication could affect the formed clot structure, since the same study by Evans et al. discovered that increasing levels of unfractionated heparin cause decreases in the measured fractal dimension (Evans et al. 2010). Previous studies have shown that the changes in clot microstructure and viscoelastic properties could indicate prothrombotic disease state, such as VTE; hence the fractal dimension that quantifies the forming clot structure 
might be used as a biomarker for disease state (Lawrence et al. 2015; Weisel \& Litvinov 2013b; Undas et al. 2009; Undas \& Ariens 2011). Lawrence et al. showed that the fractal dimension measured in blood of VTE patients is different from that of non-VTE patients, who are also receiving anticoagulant treatment (Lawrence et al. 2015).

It has been previously found that the incipient clot microstructure, characterised by the fractal dimension, acts as a template for the structure of the fully formed clot (Curtis et al. 2013). Therefore it is reasonable to assume that the incipient clot structure will influence the nonlinear and fracture properties of the fully formed mature clot.

\subsection{Nonlinear rheology}

Nonlinear rheology is the study of flow and deformation of the materials outside the linear viscoelastic range. Advanced forms of rheometrical techniques to measure nonlinear behaviour include Large Amplitude Oscillatory Shear (LAOS) which can be conducted in a strain-controlled (LAOStrain) or stress-controlled (LAOStress) manner.

Early studies in nonlinear rheology attempted to describe nonlinear behaviour by extending the linear viscoelastic moduli into the nonlinear regime, in order to categorise and characterise nonlinear behaviour (Sim et al. 2003; Hyun et al. 2002; Storm et al. 2005). However, this approach is debatable, because outside the Linear Viscoelastic Range (LVR), the measured stress or strain waveforms cease to be perfectly sinusoidal, and therefore the linear viscoelastic moduli lose their physical meaning. In addition, the linear viscoelastic moduli only account for the fundamental harmonic frequency and consequently cannot capture the rich nonlinearities of the material.

Nonlinear material behaviour can be interpreted in the time domain or in frequency domain. Fourier transform rheology well describes the onset and level of nonlinearity in terms of higher harmonic contributions (Wilhelm 2002). By the method of stress 
(Chebyshev) decomposition elastic and viscous contributions to the resulting stress can be studied (Cho et al. 2005). More recently LAOStrain and LAOStress frameworks have been developed, and new, meaningful nonlinear parameters have been introduced for describing the nonlinear properties of materials experiencing large strains or stresses (Dimitriou et al. 2013; Läuger \& Stettin 2010; Ewoldt et al. 2008). Aforementioned methods and nonlinear parameters are explained in detail within Chapter 2.6.

Within the human body, both the wall shear rate and wall shear stress can vary over a wide range, depending on the type of blood vessel. Even within the veins, the values of wall shear rate and wall shear stress can show differences of several magnitudes, due to the size, branching and number of vessels (Kroll et al. 1996). A blood clot within the blood vessel is experiencing increasing levels of shear stresses as the blood is flowing past the forming thrombus (Colace et al. 2012). Therefore, LAOStress is a suitable method for studying the nonlinear rheological behaviour of blood clots under conditions that are relevant to those encountered by clots formed in the blood vessels.

\subsection{Nonlinear rheology of fibrin}

Studying and understanding the nonlinear rheological properties of strain stiffening biopolymer gels, such as fibrin gels or blood clots, is very important. In the case of both fibrin gels and blood clots, the underlying 3-dimensional fibrin network bears the forces imposed on the clot. The underlying fibrin network deforms as blood clots are sheared by the hydrodynamic blood flow within the blood vessels (Gersh et al. 2010; Campbell et al. 2010; Flamm \& Diamond 2012), as well as due to contraction caused by the presence of platelets (Feghhi \& Sniadecki 2011; Collet et al. 2002; Campbell et al. 2008; Jawerth et al. 2013; Lam et al. 2011). Thus, understanding the role of fibrin network structure and its mechanical, especially nonlinear properties is essential, because it could explain mechanisms behind thrombotic diseases like PE. Researchers have proposed different methods in order to characterise the nonlinear rheological properties of fibrin gels as a consequence of the application of large strains and stresses. 


\subsubsection{Nonlinear characteristics of fibrin}

The fibrin network and individual fibrin fibres show some remarkable nonlinear properties, such as strain-stiffening behaviour and high extensibility. Previous studies have shown that the nonlinear behaviour of the fibrin clots arises from the fibrin network structure (Ryan et al. 1999). Both crosslinked and uncrosslinked fibrin clots display strain-stiffening behaviour under large shear deformations as illustrated by the measured differential storage modulus which increases twentyfold when the applied strain increases from 1 to 50 \% (Janmey et al. 1983; Bale \& Ferry 1988). Plasma samples in the presence of platelets showed increased clot strengths (i.e. higher storage modulus was measured) and decreased level of strain stiffening (Shah \& Janmey 1997; Jansen et al. 2013). The strain-stiffening behaviour has been detected even in the case of individual fibres within the network (Liu et al. 2006; Liu et al. 2010). Li et al. have found strong correlation between the diameter of fibrin fibres and their modulus ( $\mathrm{Li}$ et al. 2016). They proposed that the conditions, such as thrombin concentration affect the diameter of the fibrin fibres within the network as well as the stiffness of the individual fibres. The thrombin concentration also affects the resistance against fibrinolysis, in such a way that more dense clots consisting of thinner fibres are more difficult to dissolve (Li et al. 2016).

As both the fibrin fibre network and the individual fibres within the network display a strain stiffening behaviour, Hudson et al. have investigated the effect of stiffening of individual fibrin fibres on the strength of the whole fibre network, by stretching 2D fibrin networks up to the point of failure and simultaneously monitoring the strain of individual fibres with the application of combined fluorescence and atomic force microscope (Hudson et al. 2010). It was found that the strength of the network origins from the strain distribution within the system. As the network is stretched, some fibres that are more strained distribute some strain load to the surrounding less stiffer fibres, hence avoiding strain concentrations, which would weaken the structure.

The nonlinear properties of fibrin clots are also affected by the deformation history of the clot (Shah \& Janmey 1997; Münster et al. 2013; van Kempen et al. 2015). Fibrin clots without crosslinks showed a continuous change in nonlinear rheological 
response under repeated large strain loadings (Münster et al. 2013). In this case, the applied shear loading caused a delay in the occurrence of strain stiffening, which suggests that the role of crosslinks is not only to form a stiffer clot that is more resistant to fibrinolysis but also to prevent a shift in strain stiffening under continuous shear loadings (Münster et al. 2013; Weisel \& Litvinov 2013a). Chernysh et al. have suggested that fibrin is an equilibrium polymer (Chernysh et al. 2012). In the early stage of clot formation, uncrosslinked fibrin clots could be more dynamic systems than that has been previously believed, as clots can rearrange as a response to local environmental effects. The findings have also shown that in the early stage of clot formation the polymerisation is reversible. This behaviour might be relevant in the likelihood of clots to embolise.

Fibrin clots experience a negative normal stress in response to the applied shear stress (Janmey et al. 2007; Kang et al. 2009). Fibrin clots have been found to be highly extensible, as tensile experiments showed that fibrin can be stretched more than fourfold before breakage (Brown et al. 2009). The highly extensive stretching is possibly a result of protein unfolding, and leads to the densification of fibres and expulsion of water simultaneously (Purohit et al. 2011).

It has been shown in stress-controlled experiments that the fracture strain of blood clots is strongly dependent on the amount of red blood cells present, which might be important to understand the mechanism of embolism (Litvinov \& Weisel 2017; Riha et al. 1999). In a study using human plasma, the formed clots were stretched up to the point of rupture with a special measuring apparatus utilising hydraulic tension. The plasma clot underwent significant deformation during stretching; simultaneously serum was pressed out from the clot, and the eventual average rupture stress was measured as $550.5 \mathrm{mN} / \mathrm{mm}^{2}$ (Savushkin 2003).

\subsubsection{Review of testing methods for characterising fibrin nonlinearities}

Earlier studies that have investigated the strain stiffening behaviour of fibrin gels and plasma clots involved probing the frequency and strain dependence of the clot by monitoring the changes in the linear viscoelastic storage and loss moduli (Shah \& 
Janmey 1997; Riha et al. 1999). However, in the nonlinear viscoelastic range, the resulting stress is not perfectly sinusoidal, hence causing quantitative inaccuracies in the reported values of linear viscoelastic moduli (Shah \& Janmey 1997; Hyun et al. 2011).

In a study by Kang et al. mature fibrin clot (fibrin samples were left to fully form between the rheometer plates) samples were tested by the application of a constant shear strain, using a controlled strain rheometer. The resulting shear and normal stress was measured, and strain-stiffening behaviour of fibrin and negative normal stresses has been observed (Kang et al. 2009).

Kim et al. investigated the effect of compression on the mechanical and structural properties of crosslinked and uncrosslinked fibrin clots using a combined confocal microscope and rheometer (Kim et al. 2014). The formed fibrin clot was compressed vertically and the degree of compression was measured. The viscoelastic properties of the clots were measured by the application of SAOS after each compression and decompression step. The highest degree of compression applied caused a tenfold increase in the shear elastic modulus, and increase in the network density. The compressive normal stress or strain response was found to be strongly dependent on the initial elasticity of the clot and on the rate of compression, as the increase in rate and clot strength lead to steeper increase in the normal stress.

Broedesz et al. investigated the nonlinear rheological properties of extracellular fibrin gels and intracellular F-actin solutions by applying and comparing two protocols. They performed a strain ramp at different rates, and differential prestress experiments, in which SAOS was superimposed on increasing and then decreasing levels of constant stresses for 4 minutes and then released for 4 minutes. They also developed a simple material model. It was found in the prestress experiments that the rheological response rapidly returns to the initial response once the prestress is released. The linear properties were found to be unchanged even when the applied prestress was in the nonlinear range. As the level of prestress was increased and decreased, no significant hysteresis was observed, suggesting that the applied stress did not result in the unbinding of crosslinks (Broedersz et al. 2010). 
Jansen et al. studied the effects of cells on the development, stiffness and strain stiffening of fibrin clots (Jansen et al. 2013). They monitored the structural development of fibrin clots using a controlled stress rheometer and the application of SAOS. The results showed that the presence of cells affects the time evolution of the elastic modulus and increases the final stiffness of the gels. In the absence of cells, there was an immediate and quick increase in $G^{\prime}$, followed by a slower increase caused by the formation of covalent crosslinks initiated by FXIIIa, and $G^{\prime}$ levels off after 3 hours. The immediate, quick increase in $G^{\prime}$ was also observable in the presence of the cells, however it was followed by a further increase in $G^{\prime}$ as the cells spread, and the plateau in $G^{\prime}$ is reached later on, after 4 hours. The formed clot at steady state shows a nearly perfectly elastic solid behaviour, as $G^{\prime}$ is frequency independent and the value of phase angle is very low. The presence of cells increased the steady state $G^{\prime}$ threefold. The nonlinear properties of the formed clots were characterised by the application of steady prestresses superimposed on small stress oscillations, and the resulting differential (tangent) complex modulus, $K^{*}$, was measured. By investigating the effect of increasing levels of stresses on the differential (tangent) storage modulus, $K^{\prime}$, the workers showed that there are three different regions in the nonlinear range: two stiffening regions confine a small region of constant elastic modulus, in which the fibres are axially stretched. The presence of cells only influenced the linear region and the first stiffening region. The measured maximum stress was found to be unaffected by the presence or absence of cells, and only the total degree of strain stiffening (quantified as the ratio of maximum $K$ and maximum $G$ ) decreased by the addition of cells (Jansen et al. 2013).

Piechocka et al. have investigated the origin of the mechanical properties of fibrin gels in prestress and microrheology experiments (Piechocka et al. 2010). By studying the high-strain mechanics of fibrin gels, these workers identified four different regimes with increasing prestress levels in the nonlinear region. They have suggested that the initial strain stiffening behaviour, following the LVR, is due to the stretching (entropic elasticity) of the fibre network, followed by a secondary linear region, in which differential modulus, $K^{\prime}$, is constant with increasing prestresses. As the applied prestress is further increased, $K^{\prime}$ further increased, caused by the stretching (entropic elasticity) of the individual fibres, and clot fracture occurs during the 
unfolding of the fibrin monomers at maximum stress. Interestingly, they did not observed hysteresis when comparing the upward and downward prestress sweeps.

The mechanical properties of fibrin clots formed under continuous shear perturbations have been studied (Munster et al. 2013). Fibrin clots were formed under different strain amplitudes ( $0 \%$ - $90 \%$ \%); subsequently, the nonlinear properties were studied in a strain ramp experiment. Similarly to Piechocka et al. (Piechocka et al. 2010), different characteristic regimes were identified in the nonlinear region, including one region with unchanging differential modulus, $K^{\prime}$, confined by two regions in which $K^{\prime}$ increases. Compared to the clots formed under static conditions, in the case of clots formed under large perturbations the regimes and the rupture stresses shifted to larger strains. Confocal imaging of the samples revealed different clot architecture in the absence and presence of perturbations. Homogenous fibrin structure formed in static conditions, while perturbations caused two different layers in the fibrin network: one consisting of thick fibre bundles, and another with a more densely packed fibre network. The shift in the onset of the regimes and rupture stress was attributed to the structural differences, because larger perturbations resulted in larger regions with thick fibre bundles. During the strain ramp experiment, first the thick fibre bundles straightened, and then the more densely packed fibrin network stretched.

Yao et al. have utilised inertio-elastic oscillations, which are caused by the coupling of the instrument inertia and the elasticity of the material, in order to characterise the nonlinear behaviour of fibrin gels (Yao et al. 2008). They have compared the analysis of inertio-elastic oscillations (calculated the differential moduli using the ringing analysis as a response to a sudden application of a constant stress) to more conventional nonlinear rheological techniques, such as monitoring the linear viscoelastic moduli in a stress sweep, geometrical representation of the LAOS test results using Lissajous-Bowditch plots and the application of prestress experiments. They suggested that the application of inertio-elastic oscillations is a more accurate tool for nonlinear characterisation in comparison to the other methods used, as it can be used to evaluate the nonlinear elasticity and dissipation of the material at any applied stress levels. 
Liu et al. have developed an elegant technique, which combines an atomic force microscope (AFM) with a fluorescence microscope, in order to measure the mechanical properties of individual fibrin fibres in the presence and absence of FXIIIa (Liu et al. 2006). The AFM tip stretches a single fibre whilst the fluorescence microscope images this process. Both crosslinked and uncrosslinked fibres showed large degree of stretching. It was found that the crosslinked fibres can be stretched at least four times their original length. In contrast to collagen, spider silk and keratin fibres, where the presence of crosslinks causes less extensibility, the uncrosslinked individual fibres were found to be less extensible, than the crosslinked fibrin fibres. They also observed that the crosslinked fibres could recover without any irreversible damage even after stretching by 2.8 times their length, which corresponds to $180 \%$ applied strain. The whole fibrin network showed less extensibility compared to the individual fibres, this is due to the alignments of the fibres prior to stretching. These finding also suggest that the fracture of the fibrin clots is caused by the yielding of the branching points, rather than the rupture of the single fibres.

Munster et al. first performed repeated large-strain loadings on collagen and fibrin biopolymer networks, with a view to understand the behaviour of these structures within the body (Münster et al. 2013). They subjected fibrin clots to a series of sinusoidal, large-strain oscillations with constant amplitude, and after 10 cycles of oscillation the fixed strain amplitude was increased to the next level up to the point of fracture. Uncrosslinked fibrin clots showed a continuous change under repeated large strain loadings. It was also found that the applied shear loading causes a delay in the occurrence of strain stiffening, rather than weakening the network. They suggested that the stretching of fibres that causes this delay of the strain stiffening might be due to the slip of monomers within the fibres. As the strain is applied to the fibrin clot, the individual fibres within the structure that are oriented (originally, before the application of shear) in the direction of shear are becoming stretched, and their length increases, while the fibres that originally oriented perpendicular to the direction of shear are buckling. Once the strain was released, the buckled fibres returned to their original position, whist the stretched fibres became buckled, suggesting that the stretched fibres did not fully return to their original position and remained extended. As the deformation was applied again, these lengthened fibres only started contributing to the total network shear once they were taut. 
Semmrich et al. explain the importance of experimental methods when investigating the rheometrical properties of entangled F-actin solutions (Semmrich et al. 2008). In contrast to the measurements in the LVR, measurements conducted outside the LVR were found to be dependent on the experimental methods and conditions. They performed LAOS, step stressing (i.e. series of creep experiments with increasing levels of stresses) and simple shear experiments with constant strain rate using two different controlled stress rheometers. In contrast to the LAOS experiments, the stiffening was found to be reversible in the step stressing tests, suggesting no irreversible rearrangements in the network structure. In the additional shear rate experiments, the strain stiffening behaviour was also detected, and the degree of stiffening was reversible up to a critical strain value, above the critical strain irreversible network softening occurred.

\subsubsection{Models to describe the nonlinear properties of fibrin gels}

Van Kempen et al. have developed a constitutive model to describe the nonlinear behaviour of fibrin clots under Large Amplitude Oscillatory Shear (LAOS), based on a Kelvin-Voigt model and taking into account all three main nonlinear characteristics of the fibrin network, such as strain stiffening, softening effect over repeated deformation cycles and increasing viscous energy dissipation within a cycle (van Kempen et al. 2015). Their model has been extended for describing both the formation and nonlinear behaviour of blood clots (van Kempen et al. 2016). In order to account for the individual contribution of red blood cells, platelets and fibrin network to the linear and nonlinear behaviour, they conducted measurements on whole blood (WB), platelet rich plasma (PRP) and platelet poor plasma (PPP), respectively. The linear rheological features, such as clot stiffness of the formed clot, and nonlinear rheological features, such as strain stiffening, softening, and viscous dissipation were all affected, by the constituents of the samples. The highest level of clot stiffness was measured in PRP, due to the contraction caused by the platelets present. In comparison to PRP, WB samples showed lower clot stiffness, as a result of the reduced platelet contraction caused by the red blood cells. The contracting platelets are pulling on the fibrin fibres, however in the case of WB red blood cells 
(RBCs) are present in large volume, which affects the clot structure. The presence of RBCs increases the heterogeneity of the fibrin fibre network within the clot and prevents platelet binding. During fibrin polymerisation, RBCs are trapped within the forming fibres, hence altering the forming structure (Gersh et al. 2009). Whilst, lowest clot stiffness, largest level of strain stiffening and most pronounced viscous dissipation was observed for PPP.

Biopolymer gels show stress or strain-stiffening behaviour prior to the irreversible breakage of the structure. Keshavarz et al. have proposed a method to predict the critical stress and strain values associated with the failure of such materials using the Bailey's durability criteria, which describes the failure of the material due to the accumulation of irreversible damage (Keshavarz et al. 2017). The Bailey criteria have previously been successfully applied to glasses and rubbers, and they provide the material's lifespan under a certain loading process. They have also proposed a generalised failure criterion for biopolymer gels. In their study, a series of independent stress relaxation experiments were conducted instead of traditional strain sweep experiments which accumulates large total deformations. This way the initial stiffening and subsequent softening can be captured more accurately. In the step strain (stress relaxation) tests, the stress response can be divided into three regions: linear viscoelastic regime, a strain-stiffening region up to a critical, maximum stress value, and the stress decreases in the third region at larger applied strains. The first macroscopic fracture (i.e. irreversible failure) occurred at the critical stress or critical strain value.

\subsection{Clot microstructure as a potential biomarker for embolism}

Abnormal fibrin clot features, such as lower clot permeability, lower compaction, higher maximum clot absorbance and longer lysis times, have been detected in VTE patients and their relatives. It was also shown that the clots formed from blood of PE patients were characterised with greater permeability, less compaction, and shorter lysis time, compared to the clots formed from blood of individuals who only experienced DVT (Undas et al. 2009). Although this study did not reveal the mechanisms behind the abnormal clot structures, it demonstrated that fibrin clot 
properties are different between patients suffering from PE and those with DVT alone.

In another study, Martinez et al. (Martinez et al. 2014) showed that plasma clots of DVT and PE patients are different in terms of structural and viscoelastic properties. In comparison to the DVT patients, plasma clots formed from PE patient's blood showed accelerated establishment of viscoelastic properties, hence decrease in lag time and increase in the rate of viscoelastic property formation. In addition, electron microscopy revealed lower fibre density for plasma clots obtained from PE patients. Consequently, it can be assumed that the clot structure might be an indicator of PE.

Lawrence et al. found significant differences in the rheometrically derived biomarker, the fractal dimension $\left(d_{f}\right)$, that quantifies the clot microstructure between blood taken from VTE patients and non-VTE patients. The VTE patients were receiving warfarin therapy to prevent thrombosis and the non-VTE patients (control group) were receiving warfarin therapy for other reasons (i.e. atrial fibrillation or heart valve disease) (Lawrence et al. 2015). Other markers showed no significant difference between the cohorts. The study also revealed evidence for abnormal clotting in VTE patients, which might be attributed to inadequate response to anticoagulant therapy or insufficiency of other markers of coagulation, such as INR value. Fractal analysis of non-anticoagulated whole blood clots was first performed by Evans et al., who identified a normal, healthy range of fractal dimension of 1.74 $( \pm 0.07)$, and also showed that progressive anticoagulation causes a linear decrease in the value of $d_{f}\left(1.55<d_{f}<1.74\right)$ (Evans et al. 2010). As it was expected, warfarin significantly lowered the value of $d_{f}$ in the case of the non-VTE group (Lawrence et al. 2015). However, the clot microstructure in term of $d_{f}$ in VTE patients undergoing warfarin therapy was indistinguishable from the value for non-anticoagulated blood $\left(d_{f}=1.73 \pm 0.055\right)$. Therefore, the resulting clot microstructure can be considered abnormal in respect to what would be expected in anticoagulated blood. Moreover, similar levels of INR value were found for both VTE and non-VTE cohorts, which indicates that the amount of warfarin being administered to the VTE patients might not be working, or the INR value cannot detect a procoagulant state. It was also suggested, that the increased value of $d_{f}$ in anticoagulated blood might indicate increased thrombotic potential, as some previous studies have shown that some 
patients, treated with warfarin, experience recurrent VTE, despite an INR value within the normal range (Kearon et al. 2008; Thachil 2012).

During thrombus growth, the evolving mechanical properties of the clot play a pivotal role. Whether the blood vessel is partially or fully blocked, the viscoelastic properties of the clot will determine whether the clot will withstand the shear stresses caused by the blood flow or break off. However, the results are ambiguous regarding the correlation between the underlying microstructure of the clot and their fracture properties (Litvinov \& Weisel 2017), or in other words their likelihood for embolism, which is investigated in the present study. 


\section{Theory}

\subsection{Simple shear}

Rheology is the study of the flow and deformation of materials. In a material undergoing flow, its elements are deforming and the adjacent particles within the material are moving relative to each other. Based on the movement of the adjacent particles within the material, the two main flow behaviours that can be described are shear and extensional flow (Figure 2.1). The main difference between these flow types is that in shear flow the adjacent particles are moving over or past each other, whilst in extensional flow they are moving towards or away from each other (Barnes 2000; Mezger 2015).

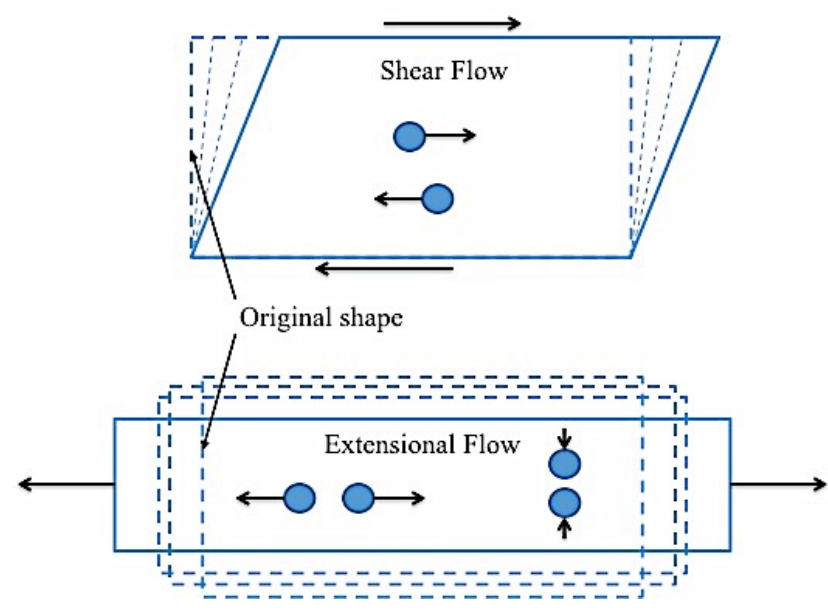

Figure 2.1 Schematic representation of (a) shear flow, in which the elements of the liquid are moving over or past each other, and (b) extensional flow, in which the elements of the liquid are moving towards or away from each other. Dashed line and arrows indicate the original shape of the material.

Rheometry refers to the measurement of the rheological properties of material and is often carried out under conditions whereby the material is subjected to shear flow. This chapter describes the theoretical framework for measuring the rheological properties of materials under shear, including oscillatory shear in both linear and nonlinear regimes. 
In a rheological apparatus, simple shear can be generated as result of a material being confined between two surfaces with a constant gap, $h$, and one plate is forced to move relative to the other plate (Figure 2.2). Shear strain, $\gamma$, is defined as the displacement divided by shear gap, and can be represented by the distortion angle, $\delta \theta$, while the shear stress, $\sigma$, is the shear force, $F$, per unit area, $A$, that generates the flow.

$$
\begin{gathered}
\gamma=\delta \theta=\frac{\delta v \delta t}{h} \\
\sigma=\frac{F}{A}
\end{gathered}
$$

Figure 2.2 illustrates that the velocity, $v$, is linearly increasing from the stationery plate $(v=0)$ to the moving plate, where the velocity is maximum. The velocity gradient is the shear rate, $\dot{\gamma}$, which can be expressed as

$$
\dot{\gamma}=\frac{v}{h}=\frac{d \gamma}{d t}
$$

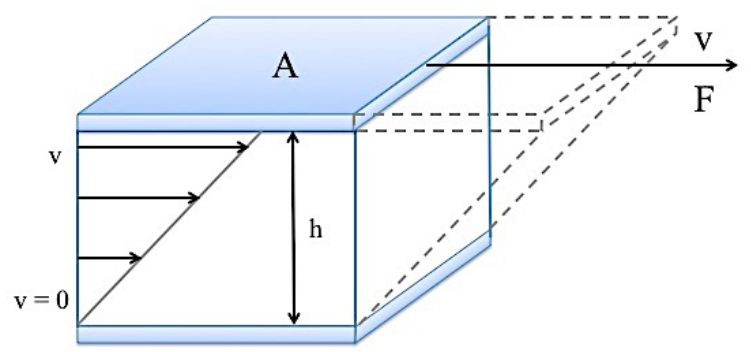

Figure 2.2 Graphical illustration of flow between parallel plates, in order to explain shear stress, shear strain and shear rate.

\subsection{Linear viscoelastic range and SAOS}

The classical rheological experiments are conducted within the Linear Viscoelastic Region (LVR) of the material. The mathematical theory of LVR is based on the Boltzmann Superposition Principle (BSP), which states that the resulting strain 
caused by a complex loading history is equal to the sum of all the individual isolated responses (Bird et al. 1977). This also suggests that the response (e.g. strain) of the material is directly proportional to the initiation signal (e.g. stress) at any time. Therefore, the material parameters recorded within the LVR; such as storage modulus and loss modulus are independent of the applied stress or strain magnitude.

In the LVR, sequential changes in strains are additive, therefore the following equation can be written (Tassieri 2016):

$$
\sigma(t)=\int_{-\infty}^{t} G\left(t-t^{\prime}\right) \dot{\gamma}\left(t^{\prime}\right) d t^{\prime}
$$

where $G(t)$ is the shear relaxation modulus and the integration is carried out over all the past times, $t^{\prime}$, up to the current time, $t$. Similarly, a constitutive equation can be written to express strain in terms of the history of the time derivative of stress $(\dot{\sigma}(t)=d \sigma(t) / d t)$, as follows:

$$
\gamma(t)=\int_{-\infty}^{t} J\left(t-t^{\prime}\right) \dot{\sigma}\left(t^{\prime}\right) d t^{\prime}
$$

where $J(t)$ is the shear creep compliance, which in a simple shear experiment, can be defined as the ratio of shear strain, $\gamma(t)$, to the magnitude of the constant shear stress, $\sigma_{0}$, which is applied at $t=0$.

$$
J(t)=\frac{\gamma(t)}{\sigma_{0}}
$$

In the case of a perfectly elastic solid $J=1 / G$, however, for a viscoelastic material $J \neq 1 / G$, due to the different experimental time patterns. The relationship between the relaxation modulus and the creep compliance can be expressed as,

$$
\int_{0}^{t} G\left(t^{\prime}\right) J\left(t-t^{\prime}\right) d t^{\prime}=t
$$


Consequently $G(t) J(t) \leq 1$.

Equations 2.2.1, 2.2.2 and 2.2.4 are convolution integrals and their mathematical properties are very useful for the solution of rheological problems. The Fourier transform (FT) of a convolution integral equals to the point-wise multiplication of the two Fourier-transformed integrand functions. Therefore, if $f(t)$ and $g(t)$ are two generic functions of the variable $t$ with a convolution integral given, the following can be expressed:

$$
w(t)=f(t) * g(t) \equiv \int_{0}^{t} f\left(t^{\prime}\right) g\left(t-t^{\prime}\right) d t^{\prime}
$$

The FT of Equation 2.2.5 is the following:

$$
\widehat{w}(\omega)=\hat{f}(\omega) \cdot \hat{g}(\omega)
$$

Where the " $\supseteq$ " symbol indicates the FT operator, which is defined as:

$$
\hat{f}(\omega)=\int_{-\infty}^{+\infty} f(t) e^{-i \omega t} d t
$$

Where $\omega$ is the angular frequency and $i=\sqrt{-1}$ is the imaginary unit.

By applying the aforementioned math, Equation 2.2.1, 2.2.2 and 2.2.4 transform as:

$$
\begin{gathered}
i \omega \hat{G}(\omega)=\frac{\hat{\sigma}(\omega)}{\hat{\gamma}(\omega)} \\
i \omega \hat{J}(\omega)=\frac{\hat{\gamma}(\omega)}{\hat{\sigma}(\omega)} \\
i \omega \hat{G}(\omega)=\frac{1}{i \omega \hat{J}(\omega)}
\end{gathered}
$$


The left side of Equation 2.2.8 and 2.2.9 represent the complex modulus, $G^{*}(\omega)$, and the dynamic compliance, $J^{*}(\omega)$, respectively.

$$
\begin{aligned}
& G^{*}(\omega)=i \omega \hat{G}(\omega) \\
& J^{*}(\omega)=i \omega \hat{J}(\omega)
\end{aligned}
$$

$G^{*}(\omega)$ and $J^{*}(\omega)$ are both complex numbers, and can be used to describe the linear viscoelastic properties of the materials.

The LVR of the material can be determined in a stress/strain sweep experiment, in which gradually increasing stress/strain levels are applied to the material in a stepwise mode (Mezger 2015). Within the LVR, the measured viscoelastic parameters, such as storage and loss moduli and phase angle are independent of the stress/strain amplitude. The transition to the nonlinear region is often associated with a deviation in the values of these parameters as the stress/strain is increasing.

\subsubsection{Oscillatory shear}

In an oscillatory shear test, one of the rheometer plates is held stationery, while the other plate oscillates in a sinusoidal manner. Sinusoidal varying strain or stress is applied, and the resulting stress or strain output is measured (Figure 2.3). Each cycle of oscillation takes a certain amount of time, and the reciprocal of that time represents the frequency of the oscillation. 


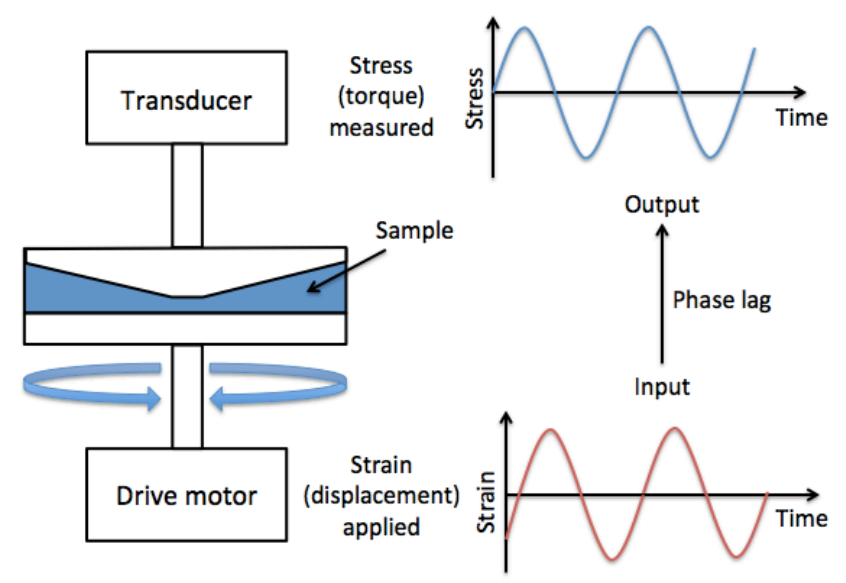

Figure 2.3 The schematic representation of a controlled strain rheometer in oscillatory mode

In oscillatory shear the applied shear strain is expressed as

$$
\gamma(t, \omega)=\gamma_{0}(\omega) \sin (\omega t)
$$

where $\gamma(t, \omega)$ is the shear strain, $\gamma_{0}(\omega)$ is the applied strain amplitude, $\omega$ is the frequency and $t$ is the time. While, the shear rate is defined as

$$
\dot{\gamma}(t, \omega)=\gamma_{0}(\omega) \omega \cos (\omega t)=\dot{\gamma}_{0}(\omega) \cos (\omega t)
$$

In order to study the linear viscoelastic properties of the materials, Small Amplitude Oscillatory Shear (SAOS), or dynamic rheological experiments are conducted. In a typical SAOS test, the material under investigation is subjected to harmonically varying sinusoidal stress or strain waveform at a constant frequency, and the resulting strain or stress waveform and the phase lag between them is measured (Ferry 1980). In the case of a Hookean solid, the applied stress/strain is in phase with the produced strain/stress, hence the phase lag is $0^{\circ}$. While, for a Newtonian liquid the applied stress/strain and measured strain/stress are out of phase by $90^{\circ}$.

Considering that sinusoidal strain is applied to the material at time, $t$, the applied strain waveform can be expressed as in Equation 2.2.13,

For a Hookean (ideal) solid the stress response can be described by the following equation. 


$$
\sigma(\omega, t)=\sigma_{0} \sin (\omega t)
$$

For Newtonian (ideal) liquid the stress response is

$$
\sigma(\omega, t)=\sigma_{0} \cos (\omega t)
$$

In the case of a viscoelastic material within the LVR, for an applied sinusoidal strain input, the shear stress response is also sinusoidal, however it is delayed by a phase angle, which is higher than $0^{\circ}$ but less than $90^{\circ}$.

$$
\sigma(\omega, t)=\sigma_{0} \sin (\omega t+\delta(\omega))
$$

where, $\delta(\omega)$ is the phase angle, which represents the lag between strain and stress.

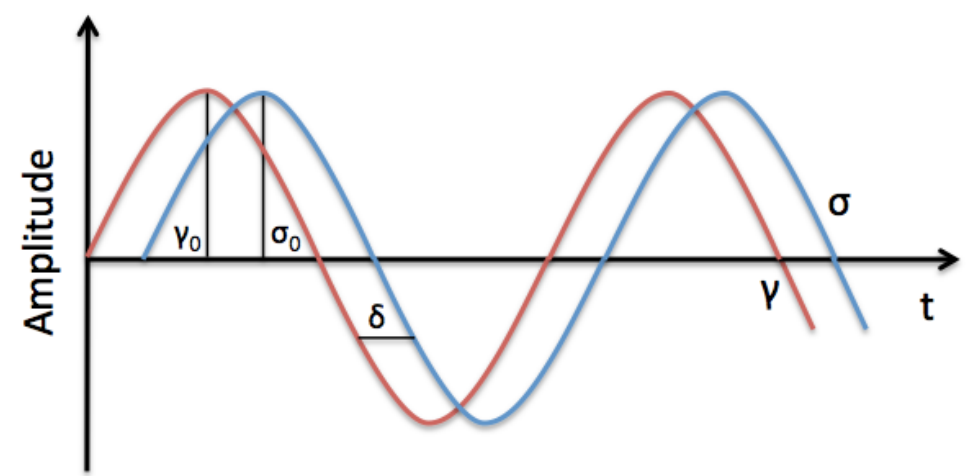

Figure 2.4 The applied sinusoidal varying strain and the resulting sinusoidal varying stress waveforms within the linear viscoelastic range

In order to solve Equation 2.2.8 some properties of specific trigonometric functions and their Fourier transforms are used. These are summarised in Table 2.1.

Table 2.1 Trigonometric functions and their Fourier transforms

\begin{tabular}{|c|c|}
\hline Function & Fourier transform \\
\hline$e^{i} \omega t$ & $2 \pi \delta_{D}(v-\omega)$ \\
\hline $\cos (\omega t)$ & $\pi\left[\delta_{D}(v-\omega)+\delta_{D}(v+\omega)\right]$ \\
\hline $\sin (\omega t)$ & $-i \pi\left[\delta_{D}(v-\omega)-\delta_{D}(v+\omega)\right]$ \\
\hline
\end{tabular}


In Table 2.1, $v$ is the Fourier frequency, and $\delta_{D}$ is the Dirac delta function. $\delta_{D}$ is equal to one if $v= \pm \omega$. However, negative values of frequency would not be physically meaningful, therefore all delta function that are triggered for negative values of $\omega$ are discarded.

The Fourier transforms of cosine and sine can be derived using Euler's formula, as follows:

$$
e^{i x}=\cos (x)+i \sin (x)
$$

Where $x=\omega t$. Therefore, it can be shown that:

$$
\begin{aligned}
& \sin (\omega t)=\frac{\left(e^{i \omega t}-e^{-i \omega t}\right)}{2 i} \\
& \cos (\omega t)=\frac{\left(e^{i \omega t}+e^{-i \omega t}\right)}{2}
\end{aligned}
$$

The complex modulus is given by the ratio of Fourier transformed stress and strain functions (Equation 2.2.8), which for two sinusoidal functions will transform as follows:

$$
\hat{\sigma}(\omega)=-i \sigma_{0} \pi \delta_{D}(v-\omega)
$$

The strain needs some manipulation prior to being Fourier transformed:

$$
\gamma(\omega, t)=\gamma_{0}(\omega)[\sin (\omega t) \cos (\delta(\omega))-\sin (\delta(\omega)) \cos (\omega t)]
$$

Therefore:

$$
\hat{\gamma}(\omega)=-\gamma_{0}(\omega) \pi \delta_{D}(v-\omega)[\sin (\delta(\omega))+i \cos (\delta(\omega))]
$$

By substituting Equation 2.2.21 and 2.2.23 into Equation 2.2.8, the following can be expressed: 


$$
G^{*}(\omega)=\frac{\hat{\sigma}(\omega)}{\hat{\gamma}(\omega)}=\frac{\sigma_{0}}{\gamma_{0}(\omega)} \frac{i}{[\sin (\delta(\omega))+i \cos (\delta(\omega))]}
$$

From Equation 2.2.24, it follows:

$$
\begin{gathered}
G^{*}(\omega)=\frac{\sigma_{0}}{\gamma_{0}(\omega)} \cos (\delta(\omega))+i \frac{\sigma_{0}}{\gamma_{0}(\omega)} \sin (\delta(\omega)) \equiv G^{\prime}(\omega)+i G^{\prime \prime}(\omega) \\
\left|G^{*}\right|=\sqrt{{G^{\prime}}^{2}+G^{\prime \prime 2}}
\end{gathered}
$$

Equation 2.2.25 provides the definition of the viscoelastic moduli. The real and imaginary parts of $G^{*}(\omega)$ are the storage, $G^{\prime}(\omega)$, and loss, $G^{\prime \prime}(\omega)$, moduli. $G^{\prime}(\omega)$ and $G^{\prime \prime}(\omega)$ provide information on the elastic and viscous characteristics of the material.

\subsection{Mechanical models of viscoelasticity}

The simplest way to describe and explain the linear viscoelastic behaviour is to use mechanical models that combine springs and dashpots (Barnes 2000). The spring represents the pure elastic element, a Hookean solid, in which case the strain is proportional to the stress. A spring shows an instantaneous strain response to an applied force, and once the force is removed, the spring immediately returns to its original form. Hence, the spring can be described by the modulus, $G$, which is the ratio of shear stress, $\sigma(t)$, to shear strain, $\gamma(t)$, and there is no time component in the formula.

$$
G=\frac{\sigma(t)}{\gamma(t)}
$$


The liquid-like behaviour, or linear viscous response can be represented by the Newtonian dashpot element. In which case the response to an applied force is delayed by the viscous resistance. The viscous element can be described by the viscosity, $\eta$, which is the ratio of shear stress and shear rate, $\dot{\gamma}(t)=d \gamma(t) / d t$.

$$
\eta=\frac{\sigma(t)}{\dot{\gamma}(t)}
$$

In the simplest representation of mechanical models, the spring and dashpot elements are connected in series or in parallel. These arrangements are known as the Maxwell model and the Kelvin-Voigt model respectively.

\subsubsection{The Maxwell model}

In the Maxwell model, the Hookean spring and Newtonian dashpot are combined in series (Figure 2.5) (Lenk 1978; Barnes 2000). This mechanical model is the simplest representation of the viscoelastic liquid behaviour.

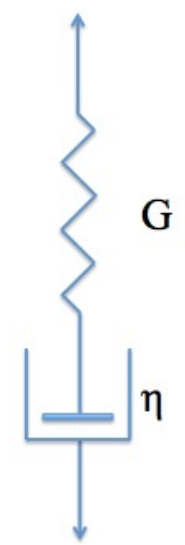

Figure 2.5 The Maxwell model

In the case of the Maxwell model, both mechanical elements experience the same stress, but different strains (Tassieri 2016).

$$
\left\{\begin{array}{l}
\gamma=\gamma_{G}+\gamma_{\eta} \\
\sigma=\sigma_{G}=\sigma_{\eta}
\end{array}\right.
$$


Where $\gamma_{G}$ and $\gamma_{\eta}$ are the strains on the string and dashpot elements, respectively. Whilst, $\sigma_{G}$ and $\sigma_{\eta}$ represent the levels stress on the spring and dashpot elements, respectively.

Using Equations 2.3.1 and 2.3.2, the formulas in Equation 2.3.3 can be rearranged as:

$$
\gamma(t)=\frac{\sigma(t)}{G}+\frac{1}{\eta} \int \sigma(t) d t+C
$$

Where constant $C$ derives from Equation 2.3.2, and its value is zero if the following boundary condition is assumed: at time, $t=0$, both stress and strain equal zero. Equation 2.3.4 is a non-homogeneous first order integro-differential equation with constant coefficients (i.e. $G$ and $\eta$ ). The solution is given by the sum of two terms:

- General solution of the related homogenous differential equation.

- Particular solution of the original, non-homogeneous integro-differential equation.

The homogeneous version can be solved as follows:

$$
\frac{\sigma(t)}{G}+\frac{1}{\eta} \int \sigma(t) d t=0
$$

Equation 2.3.5 can be differentiated and becomes:

$$
\frac{1}{G} \frac{d \sigma(t)}{d t}+\frac{\sigma(t)}{\eta}=0
$$

By separating the variables, integrating on both sides, and specifying boundary conditions (i.e. value of $\sigma(t)$ at time, $t=0$ ), the solution for Equation 2.3.6 is the following:

$$
\sigma(t)=\sigma_{0} \exp (-\lambda t)
$$

Where $\lambda=G / \eta$, which is the characteristic relaxation rate of the Maxwell model. 
Equation 2.3.4 can be solved for the particular case of a sinusoidal strain with amplitude, $\gamma_{0}$, and frequency, $\omega$, which is applied at time, $t=0: \gamma(t)=\gamma_{0} \sin (\omega t)$. In this case, the particular solution, $\sigma_{p}(t)$, can be expressed as:

$$
\sigma_{p}(t)=A \sin (\omega t)+B \cos (\omega t)
$$

In order to find constants $A$ and $B$, Equation 2.3.8 needs to be substituted into Equation 2.3.4.

$$
\begin{aligned}
\gamma_{0} \sin (\omega t)= & \frac{1}{G}[A \sin (\omega t) \\
& +B \cos (\omega t)]+\frac{1}{\eta} \int[A \sin (\omega t)+B \cos (\omega t)] d t
\end{aligned}
$$

Differentiation gives:

$$
\begin{aligned}
\omega \gamma_{0} \cos (\omega t)= & \frac{\omega}{G}[A \cos (\omega t) \\
& +B \sin (\omega t)]+\frac{1}{\eta}[A \sin (\omega t)+B \cos (\omega t)]
\end{aligned}
$$

Equation 2.3 .10 is only true for all $t$ if the two sinusoidal functions, $\sin (\omega t)$ and $\cos (\omega t)$ are the same on both sides of the equation. Therefore:

$$
\left\{\begin{array}{c}
\omega \gamma_{0}=\frac{\omega A}{G}+\frac{B}{\eta} \\
\frac{A}{\eta}-\frac{\omega B}{G}=0
\end{array}\right.
$$

Therefore:

$$
\left\{\begin{array}{l}
A=\frac{\bar{\omega}^{2} G \gamma_{0}}{\left(1+-\bar{\omega}^{2}\right)} \\
B=\frac{\bar{\omega} G \gamma_{0}}{\left(1+-\bar{\omega}^{2}\right)}
\end{array}\right.
$$


Where $\bar{\omega}=\omega / \lambda$. The solution of Equation 2.3.4 in the case of an applied sinusoidal strain is the following:

$$
\sigma(t)=\sigma_{0} \exp (-\lambda t)+\frac{\bar{\omega} G \gamma_{0}}{\left(1+\bar{\omega}^{2}\right)}[\bar{\omega} \sin (\omega t)+\cos (\omega t)]
$$

For all $t \leq 0$ the system was undeformed (i.e. $\sigma(t)=\gamma(t)=0$ for all $t \leq 0$ ), therefore it is possible to determine $\sigma_{0}$, and Equation 2.3.13 becomes:

$$
\sigma(t)=\frac{\bar{\omega} G \gamma_{0}}{\left(1+\bar{\omega}^{2}\right)}[\bar{\omega} \sin (\omega t)+\cos (\omega t)-\exp (-\lambda t)]
$$

It is important to note the single exponential decay term that vanishes for measuring times much longer than the material's characteristic time $\left(\lambda^{-1}\right)$. Therefore, in order to collect reliable data, several cycles of oscillation (i.e. a longer measuring time) needs to be performed at each measuring frequency. The length of this measuring time is unknown prior to the measurement, since the viscoelastic behaviour of the samples is yet unknown.

Alternatively, linear viscoelastic properties of the Maxwell model can be determined in the frequency domain. By performing FT of the total strain, it can be expressed:

$$
\hat{\gamma}(\omega)=\hat{\gamma}_{G}(\omega)+\hat{\gamma}_{\eta}(\omega)
$$

From Equation 2.3.1 and 2.3.2 it follows:

$$
\hat{\gamma}(\omega)=\frac{\hat{\sigma}(\omega)}{G}+\frac{\hat{\sigma}(\omega)}{i \omega \eta}=\left(\frac{1}{G}+\frac{1}{i \omega \eta}\right) \hat{\sigma}(\omega)
$$

In addition, from Equation 2.2.8 and 2.2.25, the following can be derived:

$$
G^{*}(\omega)=\frac{\hat{\sigma}(\omega)}{\hat{\gamma}(\omega)}=\frac{i \omega \eta}{1+i \omega \tau}=\frac{\tau \eta \omega^{2}}{1+\omega^{2} \tau^{2}}+i \frac{\eta \omega}{1+\omega^{2} \tau^{2}}
$$


Where $\tau=\lambda^{-1}=\eta / G$, is the characteristic relaxation time of the Maxwell model. Therefore, the Maxwell model is the mechanical representation of a viscoelastic material that has frequency dependent viscoelastic moduli.

Figure 2.6 and Figure 2.7 show the normalised storage modulus and the phase angle as a function of dimensionless frequency. It can be seen in Figure 2.6 and Figure 2.7, that as the frequency converges to zero, the storage modulus converges to zero and the phase angle converges to $90^{\circ}$. Therefore the system will tend to show more viscous behaviour at longer timescales. In contrast, as the frequency converges to infinite, the storage modulus reaches its maximum value and the phase angle converges to zero. Therefore the system tends to show more elastic behaviour at shorter timescales.

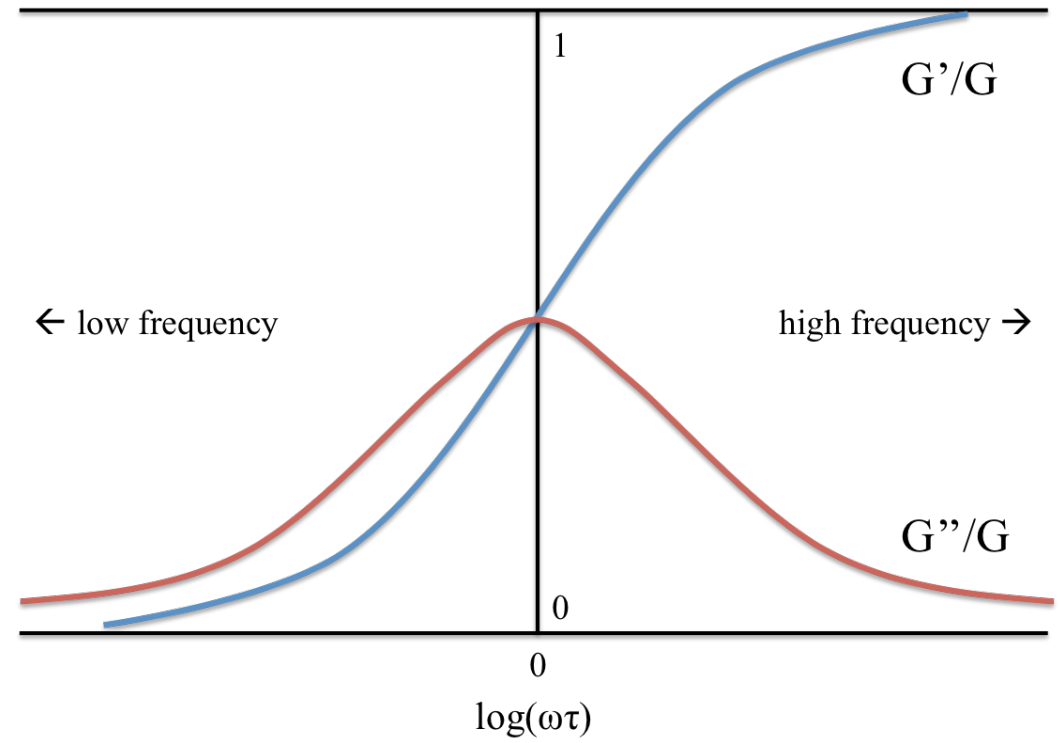

Figure 2.6 The normalised storage modulus as a function of dimensionless frequency 


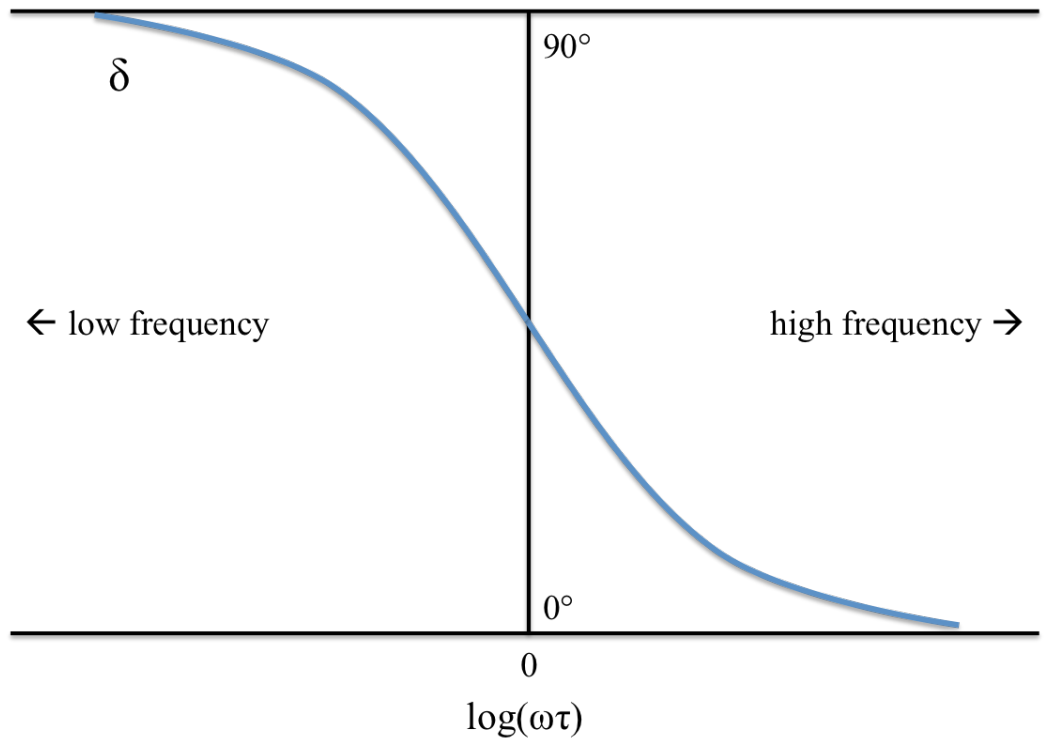

Figure 2.7 The phase angle as a function of dimensionless frequency

\subsubsection{The Kelvin-Voigt model}

In the Kelvin-Voigt model, the Hookean spring and Newtonian dashpot are connected in parallel (Figure 2.8) (Lenk 1978; Barnes 2000). This mechanical model is the simplest representation of viscoelastic solid behaviour.

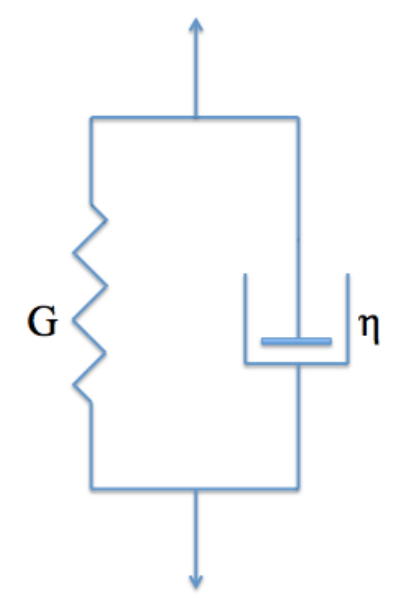

Figure 2.8 The Kelvin-Voigt model

In the case of the Kelvin-Voigt model, when stress is applied, both mechanical elements experience the same amount of strain (Tassieri 2016). 


$$
\left\{\begin{array}{l}
\gamma=\gamma_{G}=\gamma_{\eta} \\
\sigma=\sigma_{G}+\sigma_{\eta}
\end{array}\right.
$$

Using Equations 2.3.1 and 2.3.2, the formulas in Equation 2.3.8 can be rearranged as:.

$$
\sigma(t)=G \gamma(t)+\eta \dot{\gamma}(t)
$$

Equation 2.3.19 is a non-homogeneous first order differential equation with constant coefficients (i.e. $G$ and $\eta$ ). The solution is given by the sum of two terms:

- General solution of the related homogenous differential equation.

- Particular solution of the original, non-homogeneous differential equation.

The homogeneous version can be solved as follows:

$$
\eta \dot{\gamma}(t)+G \gamma(t)=0
$$

By separating the variables, integrating on both sides, and specifying boundary conditions (i.e. value of $\gamma(t)$ at time, $t=0$ ), the solution for Equation 2.3.20 is the following:

$$
\gamma(t)=\gamma_{0} \exp (-\lambda t)
$$

Where $\lambda=G / \eta$, which is the characteristic relaxation rate of the Kelvin-Voigt mechanical system.

Equation 2.3.19 can be solved for the particular case of a sinusoidal stress with amplitude, $\sigma_{0}$, and frequency, $\omega$, which is applied at time, $t=0: \sigma(t)=\sigma_{0} \sin (\omega t)$. In this case, the particular solution, $\gamma_{p}(t)$, can be expressed as:

$$
\gamma_{p}(t)=A \sin (\omega t)+B \cos (\omega t)
$$

In order to find constants $A$ and $B$, Equation 2.3.22 needs to be substituted into Equation 2.3.19. 


$$
\begin{aligned}
\sigma_{0} \sin (\omega t)= & G \gamma_{p}(t)+\eta \dot{\gamma}_{p}(t) \\
& =G[A \sin (\omega t) \\
& +B \cos (\omega t)]+\eta[A \cos (\omega t)-B \omega \sin (\omega t)]
\end{aligned}
$$

Equation 2.3.23 is only true for all $t$ if the two sinusoidal functions, $\sin (\omega t)$ and $\cos (\omega t)$ are the same on both sides of the equation. Therefore:

$$
\left\{\begin{array}{c}
\sigma_{0}=G A-\eta B \omega \\
G B+\eta A \omega=0
\end{array}\right.
$$

Therefore:

$$
\left\{\begin{array}{l}
A=\sigma_{0} \frac{G}{G^{2}-\eta^{2} \omega^{2}} \\
B=\sigma_{0} \frac{\eta \omega}{\eta^{2} \omega^{2}-G^{2}}
\end{array}\right.
$$

Therefore, the solution of Equation 2.3.19 in the case of an applied sinusoidal stress is the following:

$$
\gamma(t)=\gamma_{0} \exp (-\lambda t)+\frac{\sigma_{0}}{G} \frac{1}{\left(1-\bar{\omega}^{2}\right)}[\sin (\omega t)-\bar{\omega} \cos (\omega t)]
$$

Where $\bar{\omega}=\omega / \lambda$. For all $t \leq 0$ the system was undeformed (i.e. $\sigma(t)=\gamma(t)=0$ for all $t \leq 0$ ), therefore it is possible to determine $\gamma_{0}$, and Equation 2.3.26 becomes:

$$
\gamma(t)=\frac{\sigma_{0}}{G} \frac{1}{\left(1-\bar{\omega}^{2}\right)}\{\bar{\omega} \exp (-\lambda t)+[\sin (\omega t)-\bar{\omega} \cos (\omega t)]\}
$$

It is important to note that the single exponential decay term in Equation 2.3.27 vanishes for measuring times much longer than the material's characteristic relaxation time $\left(\lambda^{-1}\right)$. This suggests, that at each measuring frequency, oscillatory measurements require several cycles before showing a reliable result. 
Alternatively, linear viscoelastic properties of the Kelvin-Voigt model can be determined in the frequency domain by performing the Fourier transform of Equation 2.3.19.

$$
\hat{\sigma}(\omega)=G \hat{\gamma}(\omega)+\eta i \omega \hat{\gamma}(\omega)=(G+\eta i \omega) \hat{\gamma}(\omega)
$$

From Equation 2.2.8 and 2.2.25 it follows that:

$$
G^{*}(\omega)=\frac{\hat{\sigma}(\omega)}{\hat{\gamma}(\omega)}=G+i \omega \eta
$$

Equation 2.3.29 shows that the Kelvin-Voigt model is a mechanical representation of a viscoelastic material that has a frequency independent elastic modulus, $G^{\prime}(\omega)=G$, and a viscous modulus that increases linearly with the frequency, $G^{\prime \prime}(\omega)=\omega \eta$.

Figure 2.9 shows the normalised storage and loss modulus as a function of dimensionless frequency, and Figure 2.10 illustrates the phase angle as a function of dimensionless frequency in the case of the Kelvin-Voigt model. It can be seen from these diagrams, that as the frequency converges to zero, both the loss modulus and phase angle converge to zero. Therefore the system will tend to show more elastic behaviour at longer timescales. In contrast, as the frequency converges to infinite, the phase angle converges to $90^{\circ}$. Therefore the system tends to show more viscous behaviour at shorter timescales. 


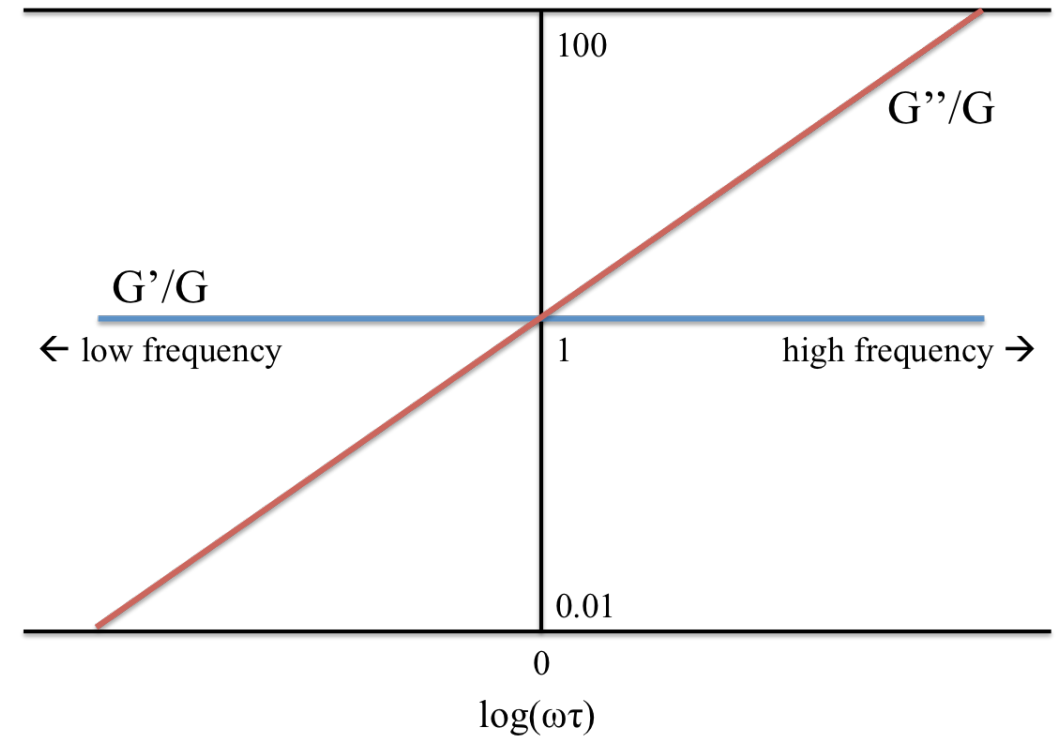

Figure 2.9 The normalised storage and loss moduli as a function of dimensionless frequency

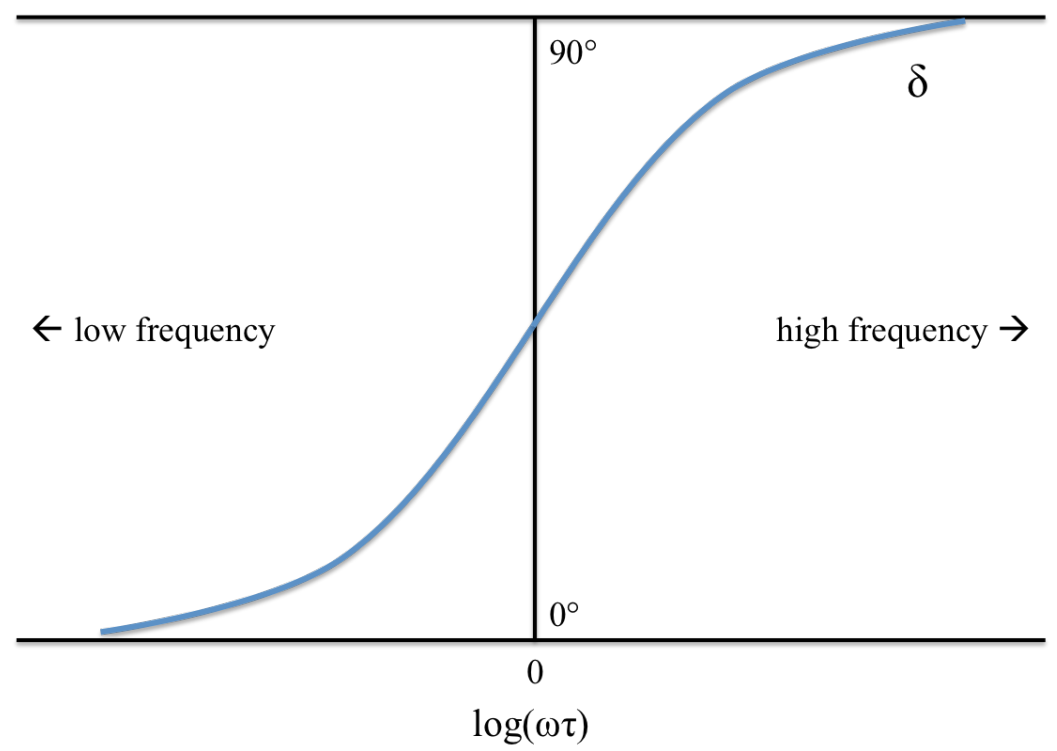

Figure 2.10 The phase angle as a function of dimensionless frequency

\subsection{Gelation}

Gelation is process in which a material transitions from liquid-like state into solidlike state. During the process of gelation, the material goes through a critical point (the GP), at which sample-spanning connectivity is established (Winter 2002). At the Gel Point, the material is in a transition between being a viscoelastic liquid (VEL) and a viscoelastic solid (VES) (Flory 1953). The two main types of gelation are chemical and physical gelation. Chemical gels are formed in chemical reactions, and 
in their 3-dimensional structures the molecules are covalently bonded. The conversion of the chemical reaction, $0 \leq p<1$ (where $p$ is the chemical conversion), can be used to follow the gelation process as it develops in time. The Gel Point occurs as a critical level of chemical conversion, $p_{c}$, is reached, at this point the size of the largest molecular cluster diverges to infinity. The material at the Gel Point is a critical gel. Prior to Gel Point, the material is soluble in good solvent, hence it is a "sol"; while past the Gel Point, it might swell in a good solvent, but it is not entirely soluble, hence it is a "gel". Therefore, the liquid-like to solid-like transition is often referred to as sol-gel transition. Physical gels are formed as sample-spanning network is created by the connectivity of super molecular aggregates. Both chemical and physical gels share similar rheological features during the process of gelation.

Some of the rheological parameters that are useful to explain the rheological behaviour at the Gel Point are the zero shear viscosity, $\eta_{0}$, which indicates the level of shear stress at low shear rates; and the equilibrium modulus, $G_{e}$, that is the limiting value of the relaxation modulus, $G(t)$, at long time scales.

$$
\begin{gathered}
\eta_{0}=\lim _{\substack{t \rightarrow \infty \\
\dot{\gamma} \rightarrow 0}} \eta(\dot{\gamma}, t) \\
\tau=\eta_{0} \dot{\gamma} \\
G_{e}=\lim _{t \rightarrow \infty} G(t)
\end{gathered}
$$

As seen on Figure 2.11, at the Gel Point the material is not a liquid anymore, and not a solid yet, as the value of zero viscosity is becoming infinitely large, while the equilibrium modulus is still zero (Winter 2002). 


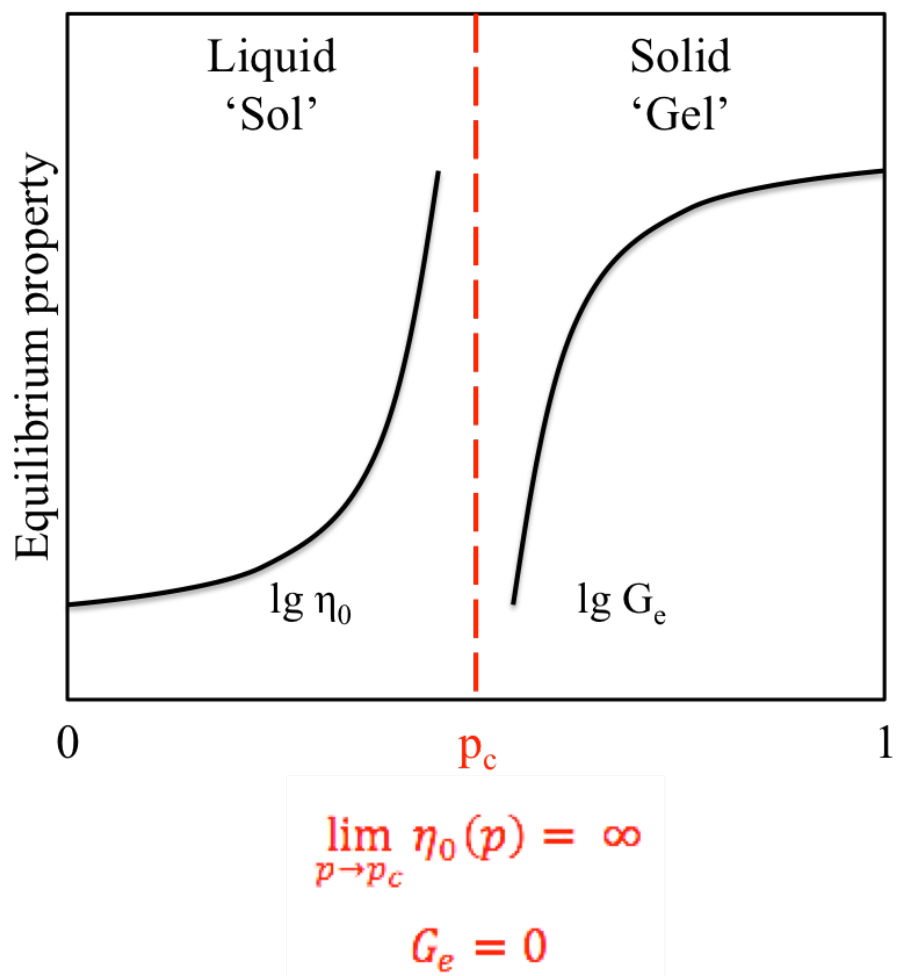

Figure 2.11 Gel Point is reached at a critical level of chemical conversion, $\mathrm{p}_{\mathrm{c}}$. At this transition point the zero shear viscosity is divergent, while the equilibrium modulus is zero.

Near the Gel Point, both the steady shear viscosity and equilibrium modulus of the 'sol' increase and show power law dependence, as the cluster size diverges, and therefore the sample-spanning network becomes stronger (Winter 2002; de Gennes 1979).

The Gel Point can be detected by the application of SAOS, as the technique allows us to probe the changes in viscoelastic behaviour as the material evolves. In the vicinity of the Gel Point, the storage modulus increases as the structure of the material strengthens. A criterion for detecting the Gel Point was suggested by Tung and Dynes based on the point at which the storage and loss moduli crossover i.e. $G^{\prime}=G^{\prime \prime}$ (Tung \& Dynes 1982). This definition holds in some cases, i.e. for PDMS polymer with balanced stoichiometry, however is not universally valid for all materials (Chambon 1986).

By successfully stopping a crosslinking reaction at certain times, Chambon was able to study the rheological properties of end-linking PDMS near the Gel Point 
(Chambon \& Winter 1985). It was found that the storage modulus scales as a power law.

$$
G^{\prime}(\omega) \sim \omega^{\alpha}
$$

The loss modulus also seemed to scale as a power law, however it was yet to be proven. The Kramer-Kronig relation validated that at the Gel Point both storage and loss moduli do indeed scale as power law (Winter \& Chambon 1986; Chambon \& Winter 1987).

$$
G^{\prime}(\omega) \sim G^{\prime \prime}(\omega) \sim \omega^{\alpha}
$$

The power law scaling in frequency of the storage and loss moduli at the Gel Point allows identifying the GP by the frequency independence of the loss tangent, $\tan \delta$.

$$
\tan \delta(\omega)=\frac{G^{\prime \prime}(\omega)}{G^{\prime}(\omega)}
$$

Figure 2.12 illustrates that the response of the material will differ as SAOS with different frequencies are applied, except at the Gel Point. 


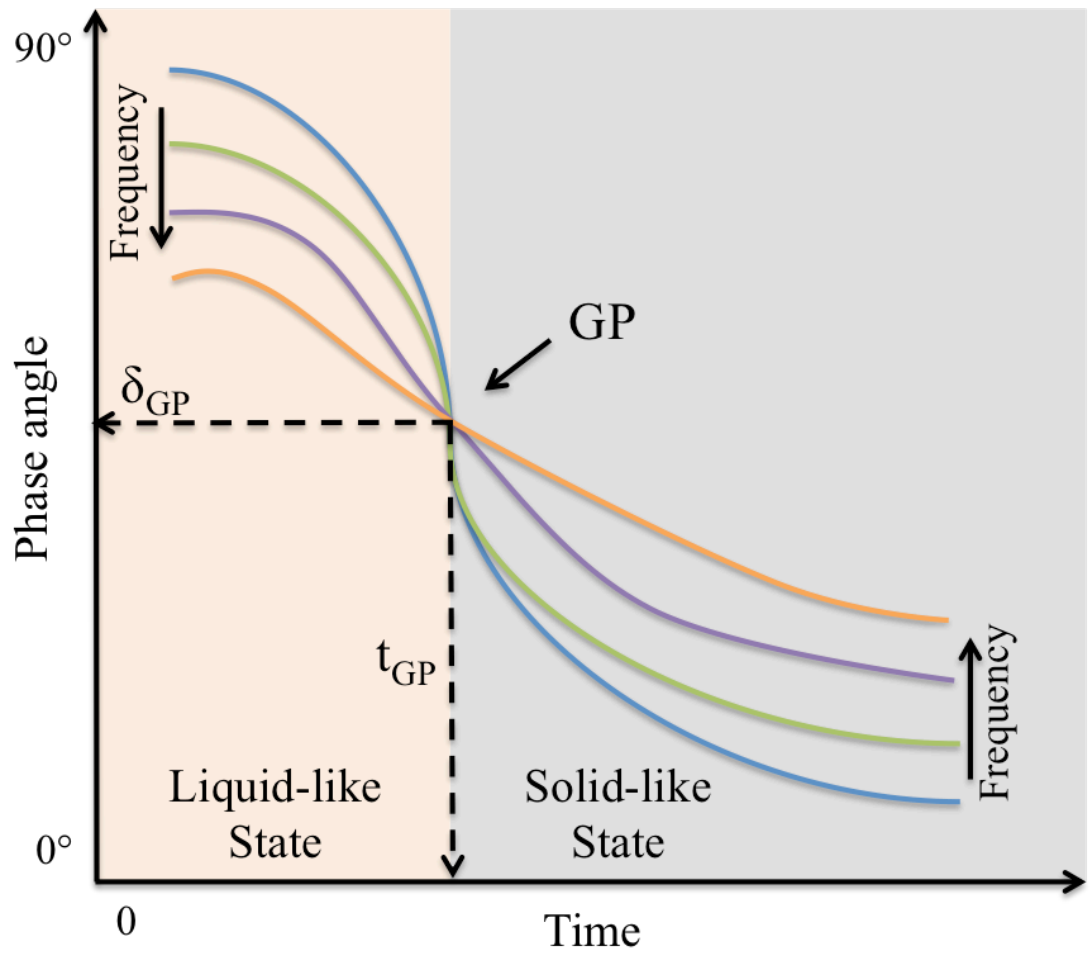

Figure 2.12 Schematic representation of a Gel Point measurement, which is a SAOS test using several different frequencies

The stress relaxation of the critical gel shows power law behaviour, hence the relaxation modulus has self-similar characteristic.

$$
G(t)=S t^{-\alpha}
$$

The relaxation behaviour of uniformly sized molecules is self-similar, fractal structure is also self-similar (Muthukumar \& Winter 1986).

The Gel Point also provides information on the underlying microstructure of the incipient sample-spanning network in terms of a fractal dimension, $d_{f}$. The relationship between $d_{f}$ and $\alpha$ was developed by Muthukumar and Winter (Muthukumar 1985; Muthukumar \& Winter 1986).

$$
\alpha=\frac{D_{e}\left(D_{e}+2-2 d_{f}\right)}{2\left(D_{e}+2\right)}
$$


Where $D_{e}$ represents the embedding space, which is equal to 3 in the case of 3dimensional space. Therefore the equation might be rearranged as,

$$
d_{f}=\frac{10 \alpha-15}{2 \alpha-6}
$$

\subsection{Nonlinear viscoelasticity}

The biological and industrial processes of many materials, such as biopolymer networks (e.g. blood clots), wormlike micelles, colloidal gels or metastable soft solids, cannot be described by steady shearing flow or linear viscoelastic deformations (Ewoldt et al. 2008). Large Amplitude Oscillatory Shear (LAOS) is used to study the nonlinear response of the materials, and therefore allows us to study the biological and industrial processes of such materials. In the case of Large Amplitude Oscillatory Strain (LAOStrain), the input sinusoidal strain amplitude $\left(\gamma_{0}\right)$ is controlled and the resulting nonlinear stress response is measured, while some recent studies have performed Large Amplitude Oscillatory Stress (LAOStress), in which sinusoidal stress amplitude $\left(\sigma_{0}\right)$ is applied and the resulting nonlinear strain is measured.

Outside the LVR, the applied strain and the resulting stress are not proportional to each other and the theory of linear viscoelasticity does not hold. Thus, the linear viscoelastic material properties, such as complex, storage and loss moduli, do not have a meaningful definition outside the LVR. Therefore, new, nonlinear material parameters have been introduced and defined, in order to interpret the results of LAOStrain or LAOStress experiments, in which the applied large strains or stresses are outside the LVR.

In a LAOStrain experiment, the input strain and strain-rate can be expressed as,

$$
\begin{gathered}
\gamma(t)=\gamma_{0} \sin \omega t \\
\dot{\gamma}(t)=\omega \gamma_{0} \cos \omega t
\end{gathered}
$$


The resulting stress can be represented in different ways. By considering the stress as an instantaneous function of the applied strain and strain-rate, in the nonlinear region, the stress response could be described by polynomial or Taylor expansion.

$$
\sigma(t)=\sum_{i=0} \sum_{j=0} c_{i j} \gamma^{i}(t) \dot{\gamma}^{j}(t)
$$

where, $c_{i j}$ are mathematical constants for the expansion of nonlinear stress as a function of strain and strain rate (Hyun et al. 2011).

After a few cycles of large strain oscillations, the resulting non-sinusoidal shear stress normally reaches a steady state (Giacomin \& Dealy 1993). In that case, the shear direction does not affect the shear stress response of a viscoelastic material, and only the sign of the shear stress changes as the sign of the shearing changes. Therefore, the shear stress must be an odd function with respect to the direction of shearing deformation. The criteria for the symmetry of the shear stress can be described with the following equation (Hyun et al. 2011),

$$
\sigma[-\gamma(t),-\dot{\gamma}(t)]=-\sigma[\gamma(t), \dot{\gamma}(t)]
$$

Considering this symmetry argument, the stress response can be describe as,

$$
\begin{aligned}
\sigma(t)=\sum_{i=1} \sum_{j=1} & {\left[C_{2 i-1,2(j-1)} \gamma^{2 i-1}(t) \dot{\gamma}^{2(j-1)}(t)\right.} \\
+ & \left.C_{2(i-1), 2 j-1} \gamma^{2(i-1)}(t) \dot{\gamma}^{2 j-1}(t)\right]
\end{aligned}
$$

The shear stress can be expressed as a function of odd higher order contributions within the nonlinear regime:

$$
\sigma(t)=\sum_{p, o d d} \sum_{q, o d d}^{p} \gamma_{0}^{q}\left[a_{p q} \sin q \omega t+b_{p q} \cos q \omega t\right]
$$


The shear stress response in LAOS contains only odd higher harmonic contributions (Hyun et al. 2011; Ewoldt et al. 2010). However, normal stress is an exception, because the sign of the normal stress stays the same when the sign of the shearing changes, and like the shear stresses, normal stresses are independent of the shear direction. Therefore, the normal stress response contains even functions. There are several criterions that must be satisfied during this mathematical derivation including no wall slip or no secondary flow (Hyun et al. 2011).

Alternatively, the total nonlinear viscoelastic stress can be described as the sum of linear viscoelastic stress and the odd higher harmonic contributions.

$$
\sigma(t)=\sum_{n=1, o d d} \sigma_{n} \sin \left(n \omega t+\delta_{n}\right)
$$

In this case the harmonic magnitude and phase angle are both dependent on the strain amplitude and frequency.

This equation can be rewritten as a Fourier series. The Fourier components are inphase and out-of-phase with applied strain, and factoring out strain amplitude define a set of nonlinear, frequency and strain amplitude dependent viscoelastic moduli.

$$
\sigma(t)=\gamma_{0} \sum_{n, o d d}\left[G_{n}^{\prime}\left(\omega, \gamma_{0}\right) \sin (n \omega t)+G_{n}^{\prime \prime}\left(\omega, \gamma_{0}\right) \cos (n \omega t)\right]
$$

Equation 2.6.6 can be rewritten as power law series, which clearly distinguish the strain amplitude and frequency dependence (Hyun et al. 2011).

$$
\sigma(t)=\sum_{n, o d d} \sum_{m, o d d}^{n} \gamma_{0}^{n}\left[G_{n m}^{\prime}(\omega) \sin (m \omega t)+G_{n m}^{\prime \prime}(\omega) \cos (m \omega t)\right]
$$




\subsubsection{FT rheology}

Fourier transform (FT) rheology quantifies the nonlinearities of a material using the relative intensities of higher harmonic contributions (Wilhelm et al. 1998; Kallus et al. 2001). The end of the linear viscoelastic range and the onset of nonlinearities can be identified precisely using this approach. It is also a useful method in order to quantify the amount of deviations from linearity (Giacomin \& Dealy 1993).

A Fourier transformation shows the inherent periodic contributions to a time dependent signal, $s(t)$, in terms of different frequencies, amplitudes and phases, and represents their contributions in a frequency dependent signal, $S(\omega)$ (Hyun et al. 2011; Wilhelm 2002). In FT-rheology, the Fourier transformation that is applied to the shear stress signal is half sided, discrete and complex. Care must be taken when data is acquired for FT-analysis. Data points have to be equally spaced in time with appropriate sampling rate, in order to minimalize any artefacts or high noise levels. Some of the techniques that are applied to improve the quality of the raw data include mechanical and electrical shielding and oversampling (Hyun et al. 2011; van Dusschoten \& Wilhelm 2001; Hilliou et al. 2004; Tassieri et al. 2016; Tassieri et al. 2018). The Fourier transformation of any real or complex time signal, $s(t)$ or frequency spectrum, $S(\omega)$, is the following (Wilhelm 2002):

$$
\begin{gathered}
S(t)=\frac{1}{2 \pi} \int_{-\infty}^{\infty} S(\omega) e^{+i \omega t} d \omega \\
S(\omega)=\int_{-\infty}^{\infty} S(t) e^{-i \omega t} d t
\end{gathered}
$$

Fourier transformation is an invertible, linear and complex mathematical operation. This linear mathematic transformation can be applied to any time dependent signal, $s(t)$. By performing Fourier transformation on a single frequency, $\omega$, signal, in the resulting Fourier spectra any peaks at frequencies that are different than $\omega$ are indications of nonlinearities; hence the nonlinearity of the material can be quantified using FT-rheology (Figure 2.13). It is also important to consider, that Fourier 
transformation is a linear transformation, therefore linear superposition of different time signals will result in linear superposition in the frequency dependent signal.

The Fourier transformation of a real time dependent signal, $s(t)$, is a complex frequency dependent spectrum, $S(\omega)$, with real, $R(\omega)$, and imaginary, $I(\omega)$, parts, as the transformation is inherently complex. In general, for the evaluation of rheological data, half-sided Fourier transformation is performed, in which case the signal is integrated in a semi-infinite region, from $t=0$ to $t=+\infty$, as the data acquisition starts at finite times (Hyun et al. 2011).

The data points in the time-domain dataset are acquired through discrete and digitalised sampling, in which case data points are recorded at a fixed increment, $t_{d w}$ (termed as dwell time or inverse sampling rate), over the total acquisition time, $t_{a q}=t_{d w} N$, where $N$ is the number of data points (Wilhelm 2002; Hyun et al. 2011). Longer acquisition time over multiple cycles decreases the line width in the FT-spectra and increases the signal-to-noise ratio. In the signal-to-noise ratio, the "signal" corresponds to the amplitude of the highest peak in the FT-spectra, and the "noise" is the standard deviation of the noise (spectra window where peaks are not present). Adequately high signal-to-noise ratio can be achieved with 5-50 cycles of oscillation, in which case the detection of even low levels of nonlinearities in the material response is attainable. The time dataset that is collected in a discrete manner is digitalised via a k-bit analog-to-digital (ADC) converter.

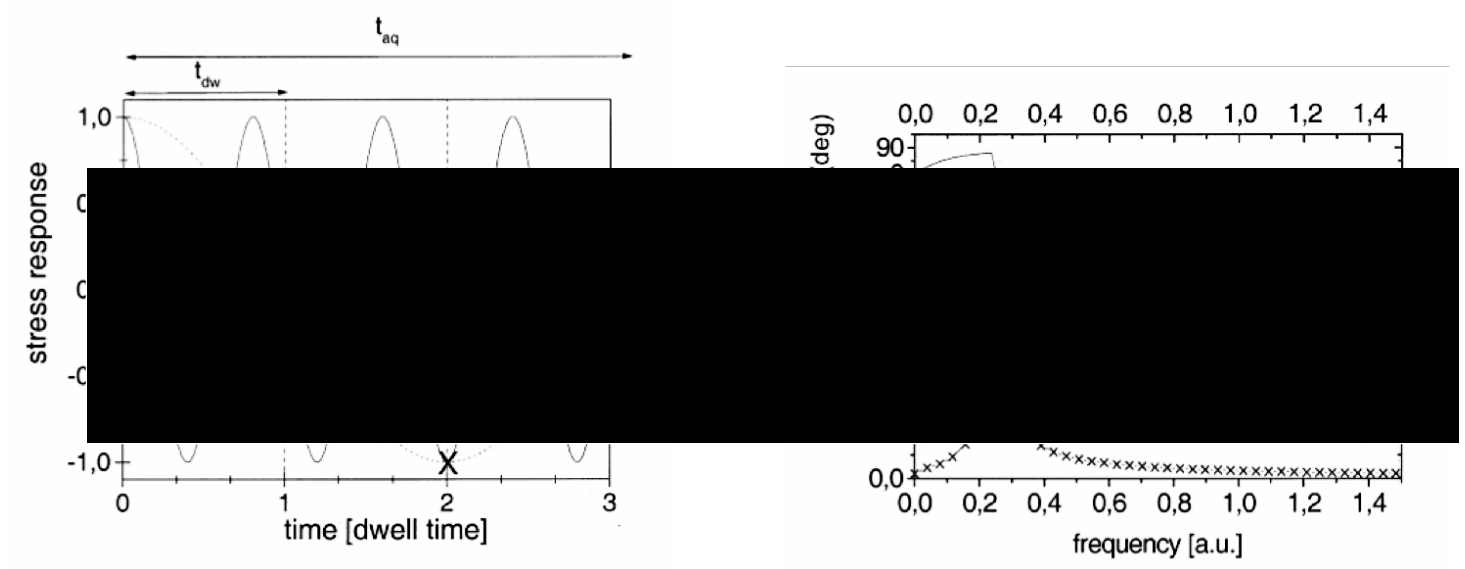

Figure 2.13 Graphical representation of the Fourier transformation (Wilhelm 2002). Time domain dataset is collected over the acquisition time, $t_{a q}$, with a certain dwell time, $t_{d w}$. The Fourier transformation of this time dependent data results in a specific frequency spectra, with spectra width of $v_{\max }$. The data acquisition time sets the minimum resolution, $\Delta v$, of the spectrum. 
FT-rheology experiment can be conducted on a modified and extended commercial controlled strain rheometer. It is challenging to capture the absolute values of the rheometer output signals, as several calibrations are required considering the magnitude, phase and frequency. Normalising the signal to the fundamental frequency, and using relative intensities can help to avoid the previously mentioned calibration requirements and any non-systematic errors. A typical FT-rheology spectrum can be seen in Figure 2.14.

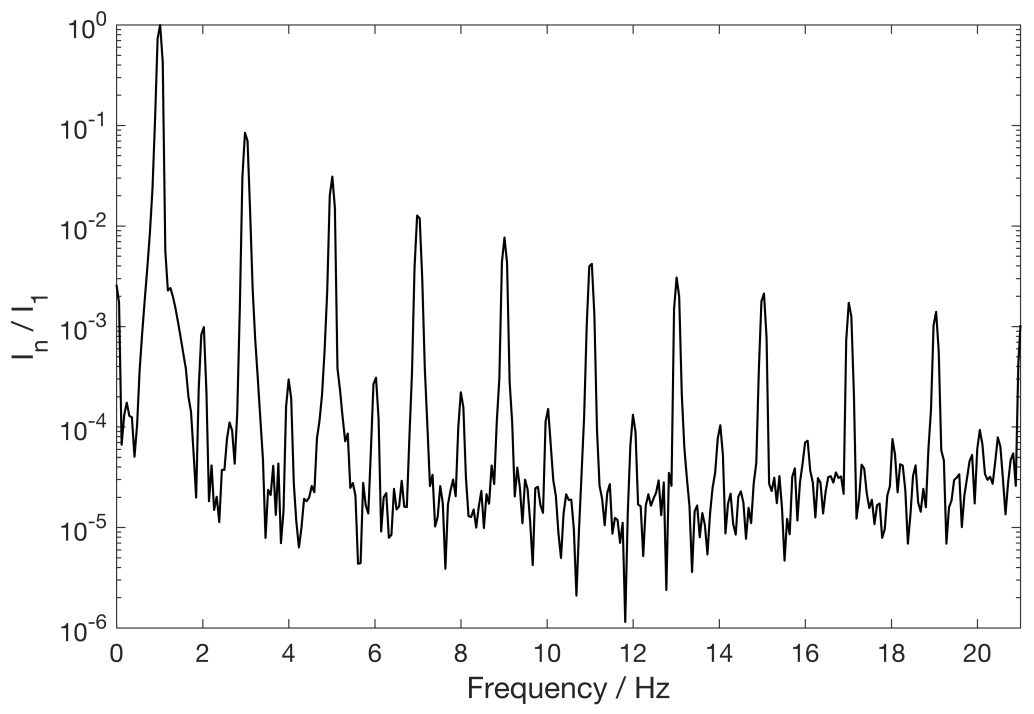

Figure 2.14 Example for FT-spectra of a fibrin clot that is tested within the nonlinear region

The higher harmonic contribution can be represented and distinguished with the FTrheology. The third harmonic contribution is the predominant contribution in the nonlinear waveform. The nonlinear region can be divided into two sub-regions: Medium Amplitude Oscillatory Shear or MAOS, and LAOS (Hyun et al. 2007). MAOS is a transition region between SAOS and LAOS, in which the third harmonic contribution shows a certain scaling. For most polymer melts, the MAOS region is present between $\gamma_{0} \approx 0.1$ and $\gamma_{0} \approx 1$, and the value of the normalised third harmonic, $I_{3 / 1}$, is very low $\left(3 \times 10^{-4}\right.$ to $\left.10^{-2}\right)$ (Hyun et al. 2011). As within the LVR, the resulting stress response is sinusoidal, the value of $I_{3 / 1}$ approaches zero, while outside the linear region, it has a finite value that increases with increasing strain amplitude. 
The normalised third harmonic ratio scales quadratically with the applied strain amplitude in MAOS, hence a new nonlinear coefficient, $Q$, was introduced (Hyun $\&$ Wilhelm 2009), which describes the material's response during the transition from linear to nonlinear regime.

$$
Q=\frac{I_{3 / 1}}{\gamma_{0}^{2}}
$$

The FT-rheology method can also be applied to quantify the degree of nonlinearity. The increasing strain amplitude result in increasing intensity of the higher harmonic contribution in the spectra (Wilhelm 2002). The nonlinear stress response can be represented by as Fourier series (Hyun et al. 2011).

$$
\begin{aligned}
& \sigma\left(t ; \omega, \gamma_{0}\right)=\gamma_{0} \sum_{n o d d}\left\{G_{n}^{\prime}\left(\omega, \gamma_{0}\right) \sin n \omega t+G_{n}^{\prime \prime}\left(\omega, \gamma_{0}\right) \cos n \omega t\right\} \\
& \sigma\left(t ; \omega, \gamma_{0}\right)=\dot{\gamma}_{0} \sum_{n o d d}\left\{\eta_{n}^{\prime \prime}\left(\omega, \gamma_{0}\right) \sin n \omega t+\eta_{n}^{\prime}\left(\omega, \gamma_{0}\right) \cos n \omega t\right\}
\end{aligned}
$$

It is assumed that the nonlinear stress response shows odd symmetry with respect to the direction of applied strain or strain rate, as in steady state, the nonlinear response of the material is the same even if the direction of the applied deformation changes. The presence of even harmonics indicates that the steady state has not been reached, secondary flows, or dynamic wall slip (Ewoldt 2009).

\subsubsection{Lissajous-Bowditch curves}

The nonlinear behaviour of materials can be graphically represented in LissajousBowditch curves (commonly called Lissajous curves). In LAOStrain, there are two types of Lissajous-Bowditch plots that represent the raw data collected during experiments: elastic Lissajous-Bowditch curves, when the measured oscillation stress is plotted as a function of applied oscillation strain, and viscous Lissajous-Bowditch 
plots, in which case the stress is plotted against strain rate. Figure 2.15 shows an example for an elastic Lissajous-Bowditch curve.

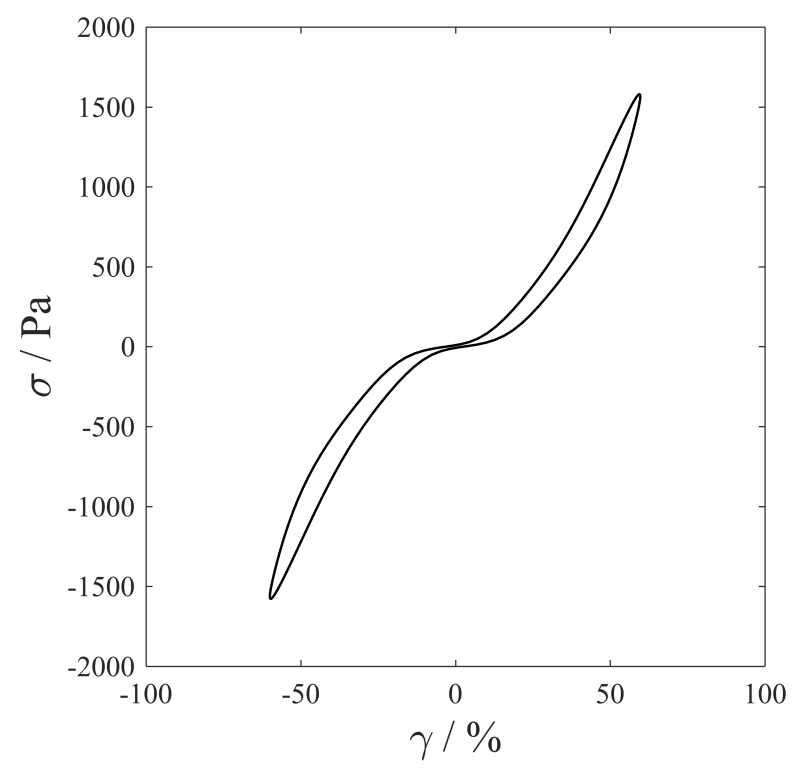

Figure 2.15 Elastic Lissajous-Bowditch plot of a fully formed, mature fibrin clot at $60 \%$ applied strain.

Tee and Dealy first attempted to quantify nonlinear behaviour using LissajousBowditch plots. They have introduced three parameters that quantify the nonelliptical shape of viscous Lissajous-Bowditch curves, and indicate the presence of nonlinearity. However, their method did not provide further physical interpretation (Tee \& Dealy 1975).

\subsubsection{Chebyshev decomposition}

The Chebyshev (stress) decomposition or geometrical interpretation approach was developed by Cho et al. (Cho et al. 2005). The method considers strain and stain rate as independent orthogonal inputs, and decomposes the measured total nonlinear stress output, $\sigma(t)$, into superposition of "elastic", $\sigma^{\prime}(x)$, and "viscous", $\sigma^{\prime \prime}(y)$, stress components using symmetry arguments (Rogers \& Vlassopoulos 2012).

$$
\sigma^{\prime} \equiv \frac{\sigma(\gamma, \dot{\gamma})-\sigma(-\gamma, \dot{\gamma})}{2}
$$




$$
\sigma^{\prime \prime} \equiv \frac{\sigma(\gamma, \dot{\gamma})-\sigma(\gamma,-\dot{\gamma})}{2}
$$

The elastic and viscous stress component can also be expressed using Fourier coefficients, such as

$$
\begin{aligned}
\sigma^{\prime} & =\gamma_{0} \sum_{n o d d} G_{n}^{\prime}\left(\omega, \gamma_{0}\right) \sin n \omega t \\
\sigma^{\prime \prime} & =\gamma_{0} \sum_{n o d d} G_{n}^{\prime \prime}\left(\omega, \gamma_{0}\right) \cos n \omega t
\end{aligned}
$$

The total measured stress is the sum of the elastic and viscous parts.

$$
\sigma(t)=\sigma^{\prime}(t)+\sigma^{\prime \prime}(t)
$$

The work of Ewoldt extended the method so that physical meaningful parameters can be derived (Ewoldt 2009). The nonlinear response of an unknown material can be characterised using Chebyshev polynomials in $x$ and $y$. Being independent and orthogonal variables, the applied strain and strain rate can be expressed as,

$$
\begin{gathered}
\gamma(t)=\gamma_{0} \sin \omega t=\gamma_{0} x(t) \\
\dot{\gamma}(t)=\left(\gamma_{0} \omega\right) \cos \omega t=\dot{\gamma}_{0} y(t)
\end{gathered}
$$

Therefore, the resulting stress can be interpreted using Chebyshev coefficients, as follows:

$$
\begin{gathered}
\sigma^{\prime}(x)=\gamma_{0} \sum_{n: o d d} e_{n}\left(\omega, \gamma_{0}\right) T_{n}(x) \\
\sigma^{\prime \prime}(y)=\dot{\gamma}_{0} \sum_{n: o d d} v_{n}\left(\omega, \gamma_{0}\right) T_{n}(y)
\end{gathered}
$$


where $T_{n}(x)$ is the $n$ th-order Chebyshev polynomial of the first kind, and $x=\gamma / \gamma_{0}$, $y=\dot{\gamma} / \dot{\gamma}_{0}$, while $e_{n}\left(\omega, \gamma_{0}\right)$ is the elastic Chebyshev coefficient and $v_{n}\left(\omega, \gamma_{0}\right)$ is the viscous Chebyshev coefficient. Within the LVR, $e_{3} / e_{1} \ll 1$, and $v_{3} / v_{1} \ll 1$. When the third-order Chebyshev polynomial has a positive contribution, higher elastic stress is measured at the maximum strain, compared to a value of stress suggested by only the first-order contribution. Therefore, $e_{3}>0$ indicates intra-cycle strainstiffening of the elastic stress, while $e_{3}<0$ shows strain-softening behaviour. Likewise, $v_{3}>0$ suggests intra-cycle shear-thickening, and $v_{3}<0$ shear-thinning of the viscous stress (Ewoldt 2009).

The Chebyshev coefficients can be related to the Fourier coefficients, as follows:

$$
\begin{gathered}
e_{n}=G_{n}^{\prime}(-1)^{(n-1) / 2} \\
v_{n}=\frac{G_{n}^{\prime \prime}}{\omega}=\eta_{n}^{\prime}
\end{gathered}
$$

where $n$ represents only odd numbers.

Alternatively, the Chebyshev decomposition can be represented graphically, as the decomposed elastic or viscous stress can be plotted as a function of strain on a Lissajous-Bowditch plot in order to assess the nonlinear elasticity and dissipation of the material.

The benefits of using Chebyshev decomposition include the both orthogonal and normalised characteristics of the Chebyshev polynomials, which also offer near optimal polynomial interpolation. The Chebyshev coefficients, $e_{n}$ and $v_{n}$, have real physical interpretations, that can be associated with classical rheological concepts, such as shear-thinning and shear-stiffening. In addition, it is possible to reconstruct temporal material response using identities for Chebyshev polynomials. 


\subsubsection{Nonlinear parameters}

Ewoldt has developed a complete, low-dimensional LAOStrain framework to quantify nonlinear viscoelasticity, which avoids any ambiguities encountered in the previously discussed methods, such as the lack of clearly defined nonlinear viscoelastic moduli (Ewoldt et al. 2008). In this new LAOStrain framework, physical meaningful nonlinear parameters have been introduced using Lissajous-Bowditch plots; and their values are reported with respect to the applied strain amplitude, $\gamma_{0}$, or strain rate amplitude, $\dot{\gamma}_{0}=\gamma_{0} \omega$, in order to show nonlinearities throughout different steady state cycles (Ewoldt et al. 2008; Ewoldt 2009).

\subsubsection{Nonlinear elastic modulus}

The first harmonic Fourier coefficient, $G_{1}^{\prime}=\omega / \pi \gamma_{0}^{2} \oint \sigma(t) \gamma(t) d t$, cannot be used as an appropriate nonlinear elastic modulus, because it does not account for the energy stored in other harmonic components, and it only describes the average elasticity in the material response that is created by a certain combination of applied large strain amplitude, $\gamma_{0}$, and frequency, $\omega$. Therefore, the first Fourier modulus is not useful to show the local elastic response of the material at small and large instantaneous strain (Ewoldt et al. 2008). Ewoldt et al. introduced a set of elastic moduli which are able to describe this local behaviour, can be geometrically represented on LissajousBowditch plots and can also be related to both Fourier and Chebyshev parameters (Ewoldt et al. 2008; Ewoldt 2009).

The minimum-strain modulus, $G_{M}^{\prime}$, is the tangent modulus at zero instantaneous strain.

$$
G_{M}^{\prime}=\left.\frac{d \sigma}{d \gamma}\right|_{\gamma=0}=\sum_{n=o d d} n G_{n}^{\prime}=e_{1}-3 e_{3}+5 e_{5}+\cdots
$$

This definition is the natural method for measuring an elastic modulus. As a sinusoidal varying strain is applied, $\gamma(t)=\gamma_{0} \sin (\omega t)$, at the point where the strain is zero, the rate of strain is at local maximum, and the viscous contribution to the 
resulting stress is locally constant, as $\frac{d \dot{\gamma}_{0}}{d t}=0$. Therefore, any change in stress is only related to elasticity (Ewoldt et al. 2008).

Large-strain modulus, $G_{L}^{\prime}$, is the secant modulus measured at maximum strain.

$$
G_{L}^{\prime}=\left.\frac{\sigma}{\gamma}\right|_{\gamma= \pm \gamma_{0}}=\sum_{n=o d d} G_{n}^{\prime}(-1)^{(n-1) / 2}=e_{1}+e_{3}+e_{5}+\cdots
$$

The definition of the large-strain modulus is also a natural way for measuring an elastic modulus, because when the strain is equal to the applied strain amplitude, $\gamma=\gamma_{0}$, the rate of strain is zero, $\dot{\gamma}_{0}=0$; hence the instantaneous viscous stress is also zero. Consequently, the total stress measured in the material is caused by the elastic behaviour of the sample (Ewoldt et al. 2008).

Within the linear viscoelastic region, at small strains $\left(e_{3} / e_{1} \ll 1\right)$, both minimum and large strain moduli are equivalent to the linear elastic modulus, $G_{L}^{\prime}=G_{M}^{\prime}=G_{1}^{\prime}=$ $G^{\prime}(\omega)$.

These nonlinear elastic moduli can be graphically represented using elastic Lissajous-Bowditch plots. The minimum-strain modulus is the slope of the tangent at zero strain, while the large-strain modulus is the gradient of the secant at maximum strain. In the linear viscoelastic range, the Lissajous-Bowditch curve is elliptical shaped, hence $G_{L}^{\prime}=G_{M}^{\prime}=G^{\prime}(\omega)$. Note, that the secant at maximum strain differs from the semi major axis of the ellipse (i.e. the complex modulus, $G^{*}$ ), except for a perfectly elastic solid, in which case the Lissajous-Bowditch plot would be a straight line (Ewoldt et al. 2008).

Outside the linear viscoelastic range, the shape of the Lissajous-Bowditch curve is distorted from an ellipse, hence $G_{L}^{\prime} \neq G_{M}^{\prime} \neq G^{\prime}(\omega)$. Figure 2.16 shows, that $G_{L}^{\prime}>$ $G_{M}^{\prime}$, suggesting intracycle strain stiffening within a cycle. The increased strain amplitude results in a decreased minimum-strain modulus, which shows intercycle softening behaviour at low strain. This behaviour could not be described only by the first order Fourier modulus (Ewoldt et al. 2008). 


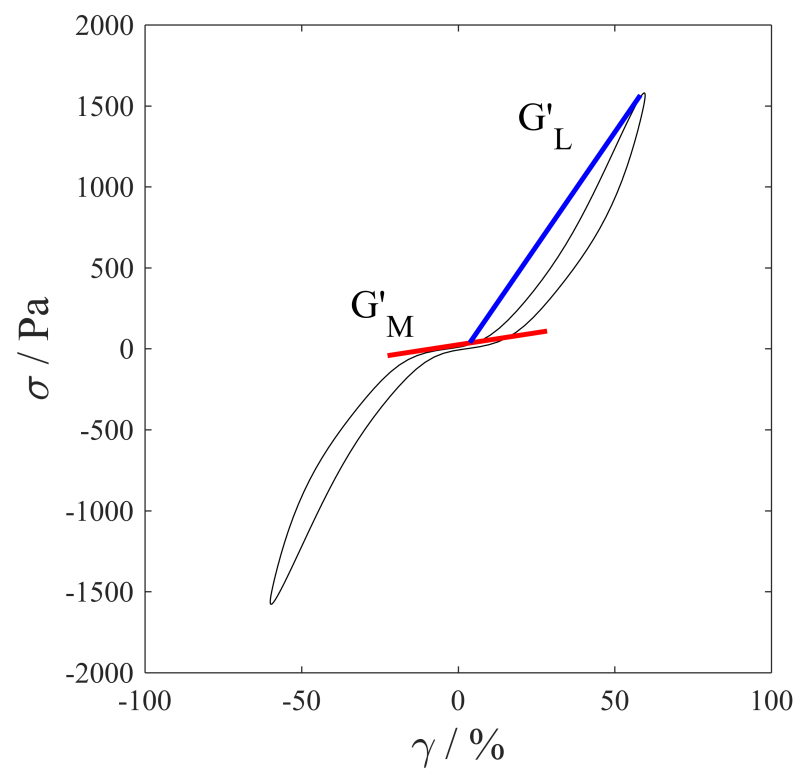

Figure 2.16 Graphical representation of the large-strain modulus and minimum-strain modulus

Another elastic modulus could be defined using the elastic stress contribution, $\sigma^{\prime}$.

$$
\left.G_{K}^{\prime} \equiv \frac{d \sigma^{\prime}}{d \gamma}\right|_{\gamma=\gamma_{0}}
$$

In this case the modulus, $G_{K}^{\prime}$, is equal to the slope of the elastic stress at maximum strain (Ewoldt et al. 2008).

\subsubsection{Nonlinear dynamic viscosity}

In oscillatory shear strain experiments, the total dissipated energy is represented by the first harmonic loss modulus, $G_{1}^{\prime \prime}$ (Giacomin \& Dealy 1993; Ewoldt et al. 2008). The total energy dissipated per cycle per unit volume is expressed as,

$$
E_{d}=\pi \gamma_{0}^{2} G_{1}^{\prime \prime}
$$

The total energy dissipation per cycle, $G_{1}^{\prime \prime}=\eta_{1}^{\prime} \omega$, only represents an average dissipation coefficient over the cycle, and does not show the local changes at the lowest and highest instantaneous shear rates. Therefore, new parameters have been 
defined that can describe the instantaneous viscosity, or coefficient of viscous dissipation, at minimum and maximum shear rates (Ewoldt et al. 2008). The minimum-rate dynamic viscosity, $\eta_{M}^{\prime}$, and large-rate dynamic viscosity, $\eta_{L}^{\prime}$, are defined as,

$$
\begin{gathered}
\eta_{M}^{\prime}=\left.\frac{d \sigma}{d \dot{\gamma}}\right|_{\dot{\gamma}=0}=\frac{1}{\omega} \sum_{n: o d d} n G_{n}^{\prime \prime}(-1)^{(n-1) / 2}=v_{1}-3 v_{3}+5 v_{5}+\cdots \\
\eta_{L}^{\prime}=\left.\frac{\sigma}{\dot{\gamma}}\right|_{\dot{\gamma}= \pm \dot{\gamma}_{0}}=\frac{1}{\omega} \sum_{n: o d d} G_{n}^{\prime \prime}=v_{1}+v_{3}+v_{5}+\cdots
\end{gathered}
$$

These parameters can also be represented graphically on a viscous LissajousBowditch plot of stress and strain rate, and their values within the linear range are equal to the dynamic viscosity, $\eta_{L}^{\prime}=\eta_{M}^{\prime}=\eta_{1}^{\prime}=\eta^{\prime}(\omega)$.

\subsubsection{Dimensionless index of nonlinearity}

Intracycle nonlinearities of the material can be quantified by comparing the values of the nonlinear elastic moduli and nonlinear dynamic viscosities. Strain-stiffening ratio, $S$, is defined as

$$
S=\frac{G_{L}^{\prime}-G_{M}^{\prime}}{G_{L}^{\prime}}=1-\frac{\sum_{n=o d d} n G_{n}^{\prime}}{\sum_{n=o d d} G_{n}^{\prime}(-1)^{\frac{n-1}{2}}}=\frac{4 e_{3} \ldots}{e_{1}-3 e_{3}+5 e_{5} \cdots}
$$

Within the linear viscoelastic range the strain-stiffening ratio is zero. Positive value of $S$ suggests intracycle strain stiffening, while it displays a negative value, intracycle strain softening can be detected (Ewoldt et al. 2008).

Similarly, shear-thickening ratio, $T$, can be derived.

$$
T=\frac{\eta_{L}^{\prime}-\eta_{M}^{\prime}}{\eta_{L}^{\prime}}=\frac{4 v_{3}+\cdots}{v_{1}+v_{3}+\cdots}
$$


The shear-thickening ratio is zero for linear viscoelastic response. $T>0$ shows intracycle shear thickening, and $T<0$ indicates intracycle shear thinning behaviour (Ewoldt et al. 2008).

\subsubsection{Stress-controlled experiments: LAOStress}

In order to characterise the nonlinear behaviour of certain materials, for example yield stress materials, Large Amplitude Oscillatory Shear Stress, LAOStress experiments are typically more effective.

Ewoldt has first attempted to extend the framework of LAOS to controlled stress rheometers (Ewoldt 2009). In the case of stress control, the elastic response of the material can be interpreted in terms of compliance

$$
J=\frac{\gamma}{\sigma} \quad\left[P a^{-1}\right]
$$

while fluidity represents the viscous response

$$
\phi=\frac{\dot{\gamma}}{\sigma} \quad\left[(P a . s)^{-1}\right]
$$

The imposed stress can be represented as

$$
\sigma(t)=\sigma_{0} \cos \omega t
$$

and in the linear viscoelastic regime the resulting strain response is

$$
\begin{gathered}
\gamma(t)=\gamma_{0} \cos (\omega t-\delta) \\
\gamma(t)=\sigma_{0}\left(J^{\prime} \cos \omega t+J^{\prime \prime} \sin \omega t\right)
\end{gathered}
$$

The magnitude of the complex compliance is 


$$
\left|J^{*}\right|=\left(J^{\prime 2}+J^{\prime 2}\right)^{1 / 2}
$$

It is important to note that $J^{\prime} \neq 1 / G^{\prime}$ and $J^{\prime \prime} \neq 1 / G^{\prime \prime}$ in general. The relationship between moduli, compliance and fluidity is the following

$$
\begin{gathered}
\left|G^{*}\right|\left|J^{*}\right|=1 \\
G^{\prime}=\frac{J^{\prime}}{J^{\prime 2}+J^{\prime 2}} \\
G^{\prime \prime}=\frac{J^{\prime \prime}}{J^{\prime 2}+J^{\prime 2}}
\end{gathered}
$$

In the nonlinear range the resulting strain and strain rate might be expressed in terms of Fourier series of higher harmonic compliance coefficients or fluidity coefficients, respectively.

$$
\begin{gathered}
\gamma(t)=\bar{\gamma}\left(\omega, \sigma_{0}\right)+\sigma_{0} \sum_{n o d d}\left\{J_{n}^{\prime}\left(\omega, \sigma_{0}\right) \cos n \omega t+J_{n}^{\prime \prime}\left(\omega, \sigma_{0}\right) \sin n \omega t\right\} \\
\dot{\gamma}\left(t ; \omega, \gamma_{0}\right)=\sigma_{0} \sum_{n \text { odd }}\left\{-\phi_{n}^{\prime \prime}\left(\omega, \gamma_{0}\right) \sin n \omega t+\phi_{n}^{\prime}\left(\omega, \gamma_{0}\right) \cos n \omega t\right\}
\end{gathered}
$$

where $J_{n}^{\prime}, J_{n}^{\prime \prime}$ are compliances and $\phi_{n}^{\prime}, \phi_{n}^{\prime \prime}$ are fluidities, and these coefficients are related by $\phi_{n}^{\prime \prime}=n \omega J_{n}^{\prime}$, and $\phi_{n}^{\prime}=n \omega J_{n}^{\prime \prime}$. The term $\bar{\gamma}\left(\omega, \sigma_{0}\right)$ is the zero harmonic accounting for the possibility of the output strain signal not being centred about zero (Ewoldt \& Bharadwaj 2013). The first harmonic measures represent a cyclic average, while physical interpretation does not currently exist for the third-harmonic measures. Therefore, it might be more useful to investigate the application of Chebyshev coefficients.

Similarly to the stress decomposition in LAOStrain (Cho et al. 2005), strain decomposition can be interpreted in LAOStress, and the measured strain response 
might be decomposed into two components (Ewoldt \& Bharadwaj 2013). First, the harmonic stress input should be made dimensionless as

$$
x(t)=\frac{\sigma(t)}{\sigma_{0}}=\cos \omega t
$$

Assuming that the elastic and viscous strain rate are both instantaneous functions of stress, the strain response can be decomposed into apparent elastic and viscous components

$$
\gamma=\gamma^{e}(\sigma)+\gamma^{v}(\sigma)
$$

The apparent elastic and viscous strain components can be expressed as (Ewoldt \& Bharadwaj 2013):

$$
\begin{aligned}
& \gamma^{e}(t)=\sigma_{0} \sum_{n o d d} J_{n}^{\prime}\left(\omega, \sigma_{0}\right) \cos n \omega t \\
& \gamma^{v}(t)=\sigma_{0} \sum_{n o d d} J_{n}^{\prime \prime}\left(\omega, \sigma_{0}\right) \sin n \omega t
\end{aligned}
$$

and the strain rate can be expressed as

$$
\dot{\gamma}=\dot{\gamma}^{e}(\sigma)+\dot{\gamma}^{v}(\sigma)
$$

The Chebyshev representation of the elastic and viscous strain components is

$$
\begin{gathered}
\gamma^{e}(t)=\sigma_{0} \sum_{n o d d} J_{n}^{\prime}\left(\omega, \sigma_{0}\right) \cos n \omega t=\sigma_{0} \sum_{n: o d d} c_{n} T_{n}(z) \\
\dot{\gamma}^{v}(t)=\sigma_{0} \sum_{n o d d} n \omega J_{n}^{\prime \prime}\left(\omega, \sigma_{0}\right) \cos n \omega t=\sigma_{0} \sum_{n: o d d} f_{n} T_{n}(z)
\end{gathered}
$$


where $c_{n}$ stands for compliances and $f_{n}$ stands for fluidities. There is a direct relationship between the Fourier and Chebyshev coefficients

$$
\begin{gathered}
c_{n}=J_{n}^{\prime}\left(\omega, \sigma_{0}\right) \\
f_{n}=n \omega J_{n}^{\prime \prime}\left(\omega, \sigma_{0}\right)
\end{gathered}
$$

$c_{n}$ are the Chebyshev compliance coefficients. Positive value of $c_{3}$ means stress softening and a negative value of $c_{3}$ means stress stiffening. $f_{n}$ are the Chebyshev fluidity coefficients. Positive value of $f_{3}$ means stress thinning and a negative value of $f_{3}$ means stress thickening (Dimitriou et al. 2013).

Similarly to large and minimum strain moduli, nonlinear compliances can be derived.

$$
\begin{gathered}
J_{1}^{\prime}=\frac{\omega}{\pi \sigma_{0}^{2}} \oint \gamma(t) \sigma(t) d t \\
\left.J_{M}^{\prime} \equiv \frac{d \gamma}{d \sigma}\right|_{\sigma=0}=\sum_{n \text { odd }}(-1)^{(n-1) / 2} n J_{n}^{\prime}=\sum_{n \text { odd }}(-1)^{(n-1) / 2} n c_{n} \\
\left.J_{L}^{\prime} \equiv \frac{\gamma}{\sigma}\right|_{\sigma= \pm \sigma_{0}}=\sum_{n \text { odd }} J_{n}^{\prime}=\sum_{n \text { odd }} c_{n}
\end{gathered}
$$

Where $J_{L}^{\prime}$ is the large stress elastic compliance, $J_{M}^{\prime}$ is the zero stress elastic compliance. $J_{L}^{\prime}$ and $J_{M}^{\prime}$ can also be graphically represented in a Lissajous-Bowditch plot (Figure 2.17). It is important to note that there is no general interrelation between the stress-dependent nonlinear compliances $J_{n}^{\prime}$ and the nonlinear moduli $G_{n}^{\prime}$, and there is no interrelation between the nonlinear parameters such as $J_{M}^{\prime}$ and $G_{M}^{\prime}$. 


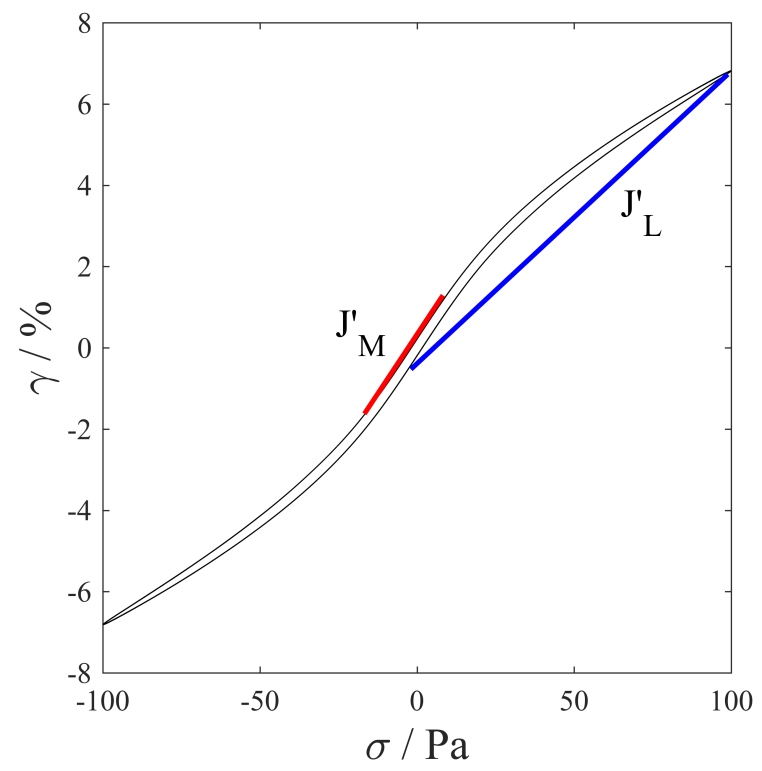

Figure 2.17 Graphical representation of the large stress elastic compliance and zero stress elastic compliance

It is also possible to define a third nonlinear parameter (Dimitriou et al. 2013):

$$
\left.J_{K}^{\prime} \equiv \frac{d \gamma^{\prime}}{d \sigma}\right|_{\sigma=\sigma_{0}}
$$

Similarly to stiffening ratio, $S$, in the case of LAOStrain, a ratio describing the change in compliance, $R$, can be defined in LAOStress (Dimitriou et al. 2013):

$$
R \equiv \frac{J_{L}^{\prime}-J_{M}^{\prime}}{J_{L}^{\prime}}=\frac{4 c_{3}-4 c_{5}+8 c_{7}-8 c_{9}+\cdots}{c_{1}+c_{2}+c_{3}+\cdots}
$$

While the nonlinear fluidities can be defined as

$$
\begin{gathered}
\phi_{1}^{\prime}=J_{1}^{\prime \prime} \omega=\frac{\omega}{\pi \sigma_{0}^{2}} \oint \sigma(t) \dot{\gamma}(t) d t \\
\left.\phi_{M}^{\prime} \equiv \frac{d \dot{\gamma}}{d \sigma}\right|_{\sigma=0}=\sum_{n \text { odd }}(-1)^{\frac{n-1}{2}} n^{2} \omega J_{n}^{\prime \prime}=\sum_{n \text { odd }}(-1)^{(n-1) / 2} n f_{n}
\end{gathered}
$$




$$
\left.\phi_{L}^{\prime} \equiv \frac{\dot{\gamma}}{\sigma}\right|_{\sigma= \pm \sigma_{0}}=\sum_{n o d d} n \omega J_{n}^{\prime \prime}=\sum_{n o d d} f_{n}
$$

where $\phi_{L}^{\prime}$ is the Large stress fluidity, and $\phi_{M}^{\prime}$ is the Zero stress fluidity.

The LAOStress framework is very useful for studying materials, which fundamentally respond to the stress input, and it would also allow studying the material's response to nonlinear stresses using a stress control rheometer.

The presence of inertia forces have to be carefully considered in the case of large amplitude oscillations, since it is the sample stress, which must be a single-harmonic sinusoidal input, rather than the instrument torque. The instrument torque may be sinusoidal, but inertia artefacts combined with a nonlinear strain response will cause a non-sinusoidal sample stress. This can be addressed by avoiding inertia artefacts, i.e. using sufficiently low frequencies, or using very precise feedback control, which accounts for this phenomena to provide a sinusoidal sample stress (Ewoldt 2009).

In the linear viscoelastic range, the material properties measured by strain-controlled and stress-controlled tests are interchangeable, as interrelations exist between compliances and moduli. However, for a nonlinear viscoelastic response it is unknown if a direct relation exists between nonlinear compliances and nonlinear moduli. Hence, nonlinear data obtained from controlled stress and controlled strain rheometers may not be interchangeable, and may therefore provide distinct information. 


\section{Methodology - Experimental apparatus}

\subsection{Rheometry}

\subsubsection{ARES-G2 controlled strain rheometer}

The ARES-G2 (TA Instruments, UK) is a controlled strain rheometer (or mechanical spectrometer), which is capable of applying the commanded dynamic (sinusoidal) strain deformations, steady shear strain, or a combination of both dynamic and steady shear to the sample, while the resulting torque that is generated by the sample is measured. The lower plate of the ARES-G2 rheometer is moving as the motor generates the commanded shear strain; and the resulting torque is measured independently by the transducer, on the stationery, upper part of the geometry. Controlled strain rheometers are often referred to as Separate Motor and Transducer (SMT) rheometers.

The ARES-G2 rheometer is controlled through a computer that is installed with TA Instruments Trios software, which allows the rheometer operator to perform any calibrations, set up experimental procedures, and enter the environmental conditions, such as operating temperature or gap size. It runs the experiments and stores the measured data. The Trios LAOS software package also allows the user to analyse the nonlinear viscoelastic data that is collected using the ARES-G2.

The ARES-G2 rheometer consists of several components, such as motor, stage, transducer, keypad, touch screen display, environmental control unit, and measuring geometries (Figure 3.1). 


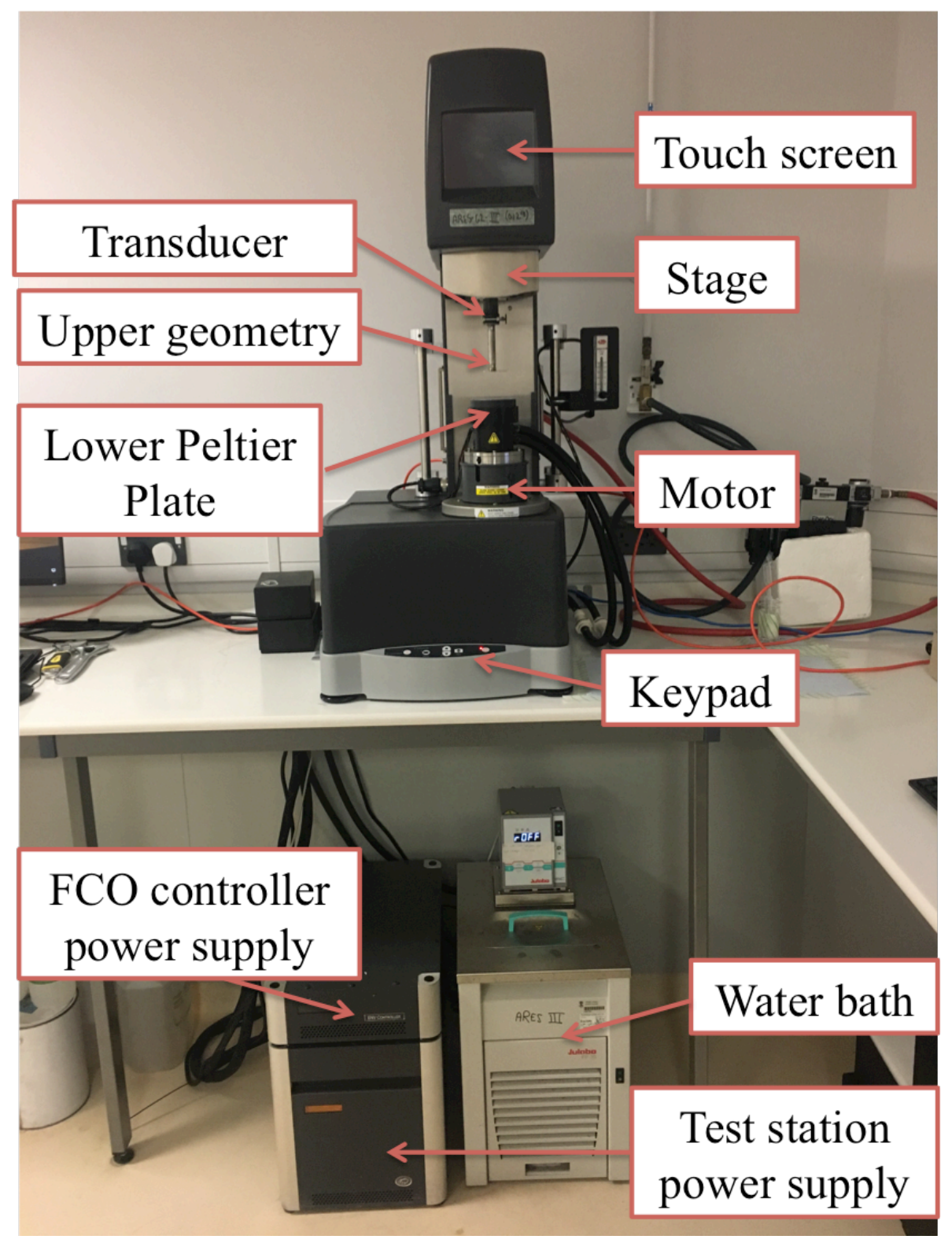

Figure 3.1 Picture of the ARES-G2 rheometer. Arrows indicate the various components.

The motorised stage supports the transducer. It can be commanded to move up or down with a certain rate, for loading or removing the sample, by pressing the keypad on the front of the rheometer test station, using the touch screen above the stage, or through the Trios software.

As an alternative to using the Trios software, the rheometer can be controlled using the keypad and touch screen of the instrument. The keypad allows the user to turn on, turn off, or check the status of the environmental control unit, transducer and motor. While with the use of touch screen zero gapping, sample loading or 
temperature setting can be performed. It also shows the current torque, normal force, gap, temperature, and status of the instrument.

The ARES-G2 is equipped with a Force Rebalance Transducer (FRT) for detecting torque and normal force generated by the sample over a wide dynamic range. The transducer shaft is kept at a fixed position by the servo control systems, in both angular and axial directions, and the torque or normal force that is required to maintain this null position of the transducer are measured as signals. The transducer shaft is held stationery, which means that instrument inertia contributions to the measured force and torque are negligible.

The input strain is generated by the air bearing motor, or actuator. The modes of operation include dynamic (sinusoidal) mode, steady (constant rotational rate) mode, or step strain and step stress mode. High resolution optical encoder monitors the position of the motor and the velocity feedback. The lowest limits of the recordable angular displacements are $0.5 \mu \mathrm{rad}$ in dynamic motion and 30 to $40 \mathrm{nrad}$ in transient motion.

The lower plate is fitted with a Peltier unit that controls the plate temperature and is capable of operating in a working range of $-40{ }^{\circ} \mathrm{C}$ to $180{ }^{\circ} \mathrm{C}$ with a resolution of 0.05 ${ }^{\circ} \mathrm{C}$. The desired level of heating or cooling is attainable using a solid-state thermoelectric pump that contains P-type and N-type semiconductor devices arranged in series, and by controlling the direction of electric currents. A water bath is connected to the Peltier plate in order to remove the excess heat generated by the elements of the unit (TA Instruments 2014).

\subsubsection{AR-G2 controlled stress rheometer}

The AR-G2 (TA Instruments, UK) is a controlled stress rheometer, which is capable of applying the commanded stress, and the resulting deformation or strain of the sample is measured. The lower plate of the AR-G2 rheometer is fixed; and the top part of the measuring system is allowed to rotate to create the required stress input by applying a certain level of torque generated by the induction motor. Controlled stress 
rheometers are often referred to as Combined Motor and Transducer (CMT) rheometers.

Similarly to the ARES-G2 rheometer, the AR-G2 controlled stress rheometer is also controlled through a computer that is installed with TA Instruments Trios software. Instrument and geometry calibrations, rotational mapping, setting up experimental procedures, and environmental conditions can be implemented in Trios. However, the Trios LAOS software package is not yet available for analysing the nonlinear viscoelastic data that is collected using the AR-G2.

The temperature is controlled via the fixed, lower Peltier plate unit, which operates between the range of $-40{ }^{\circ} \mathrm{C}$ to $200{ }^{\circ} \mathrm{C}$ with the accuracy of $0.1{ }^{\circ} \mathrm{C}$. The upper part of the geometry can rotate, and it is attached to a thin spindle, which is located in the centre of the shaft.

The AR-G2 rheometer utilises a magnetic bearing that supports the rotating shaft, which is attached to the upper geometry. The rheometer shaft is equipped with an iron thrust plate, which is held in position by the upper and lower electromagnetic actuators. The transducer is located above the upper actuator; it detects the horizontal position of the rheometer shaft. In order to maintain the position of the shaft, the actuators are supplied with the required current.

Earlier controlled stress rheometer models used an air bearing. In contrast to the air bearing system, the magnetic bearing creates less friction, since the gaps above and below the thrust plate are much wider. This improvement allows more accurate rotational mapping and measurements in lower torque ranges (as low as $0.01 \mu \mathrm{Nm}$ ), which are suitable for lightly structured materials (Costello n.d.). 


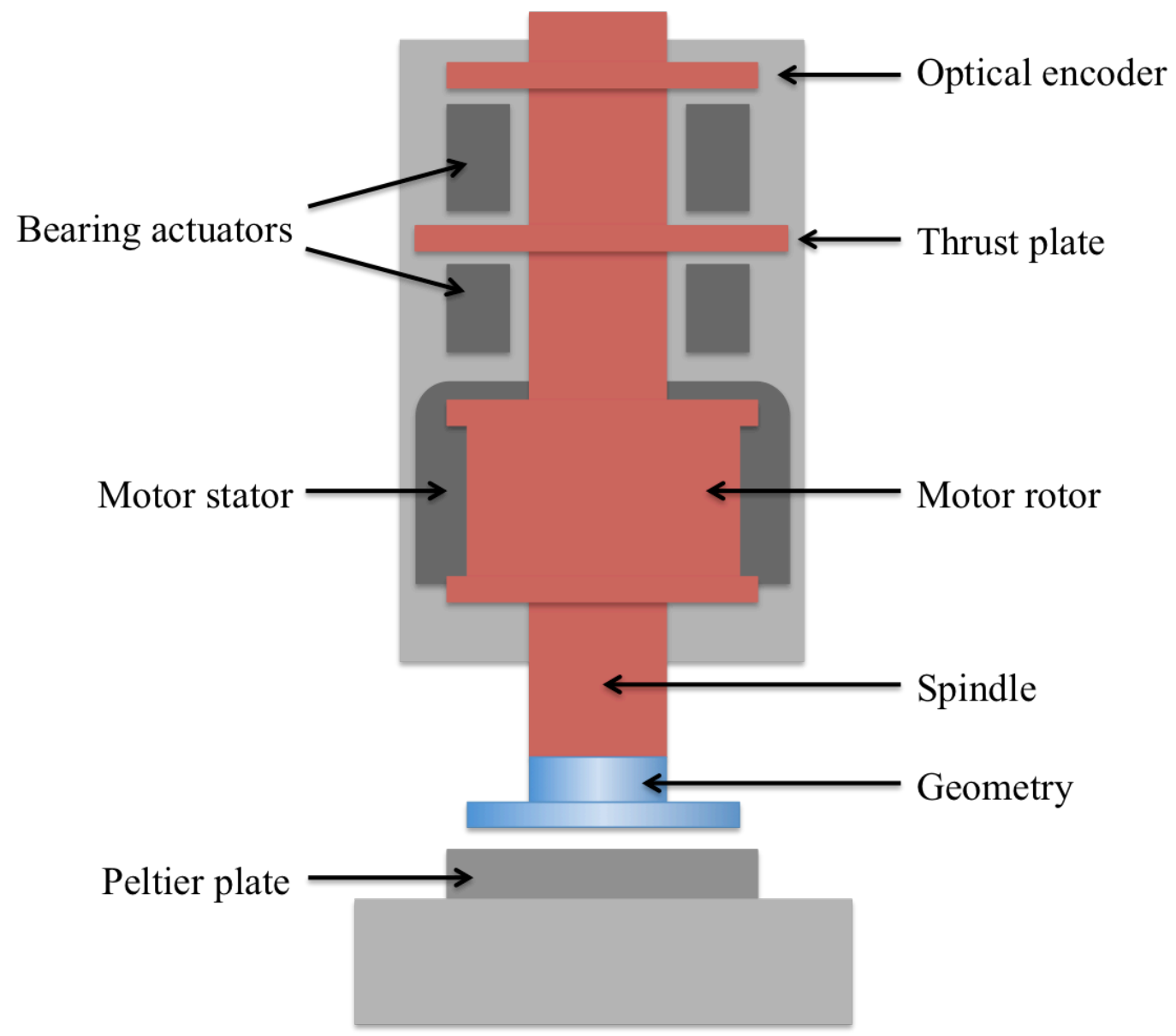

Figure 3.2 Schematic representation of the cross section of the AR-G2 rheometer. Grey colours indicate the stationary elements of the rheometer.

The optical encoder records the angular displacement caused by the response of the sample, which is then converted into strain by the rheometer software. The optical encoder is located in the upper part of the rheometer head, and it operates with a photocell that traces any change in light pattern cause by the position of two discs between a light source and the photocell. This allows measuring angular displacements as low as $25 \mathrm{nrad}$.

\subsubsection{Rheometer geometries}

Stainless steel, aluminium and acrylic are the most commonly used as geometry materials. The geometry should be as light as possible, in order to minimise the inherent inertia effects. However, instrument inertia effects are negligible in the case of a controlled strain rheometer, since the motor generates the applied strain, and the resulting stress is measured independently by the transducer, which is held 
stationery. The geometry should be chemically resistant to the test material so that corrosion problems can be avoided. The advantages and limitations of the most frequently used materials are the followings:

- Stainless steel: Compared to the other geometry materials, it is relatively heavy, but it is suitable for the majority of the test samples, it has a low thermal expansion coefficient and robust enough to withstand heavy use.

- Aluminium: The inertial effects are small, since it is relatively light, but it has higher thermal expansion coefficient and lower chemical resistance.

- Plastic: Plastic geometries are usually constructed from acrylic, polycarbonate and rigid PVC. These geometries are transparent, which allows the visual observation of the test sample. In addition, plastic geometries are lighter than metallic geometries; therefore in the case of controlled stress rheometers, contributions to instrument inertia are minimised. However, they have limited chemical compatibility and cannot be used at high temperatures.

\subsubsection{Parallel plate}

The parallel plate geometry consists of two flat plates in parallel to each other. Parallel plates are available with different diameters. This configuration is suitable for measuring samples containing particles or aggregates. The advantage of this geometry is that the gap can be set for any distance, which eliminates the problems related to particle size (i.e. in the case of cone and plate geometry, the particles could lodge under the truncation of the cone leading to an artificially elevated stress) and allows testing the wall slip effects. However, the greatest disadvantage of the parallel plate system is that the stress varies across the diameter. The material at the edge experiences the greatest strain and it has the greatest influence on the test. Therefore significant errors can occur if the test material is evaporating (as evaporation occurs at the edge of the plate) (Mezger 2015).

The shear stress and shear rate factors with respect to the rim:

$$
\dot{\gamma}=F_{\dot{\gamma}} \omega=\frac{R}{D} \omega
$$




$$
\sigma=F_{\sigma} M=\frac{2}{\pi R^{3}} M
$$

Figure 3.3 shows the schematic of a parallel plate system.
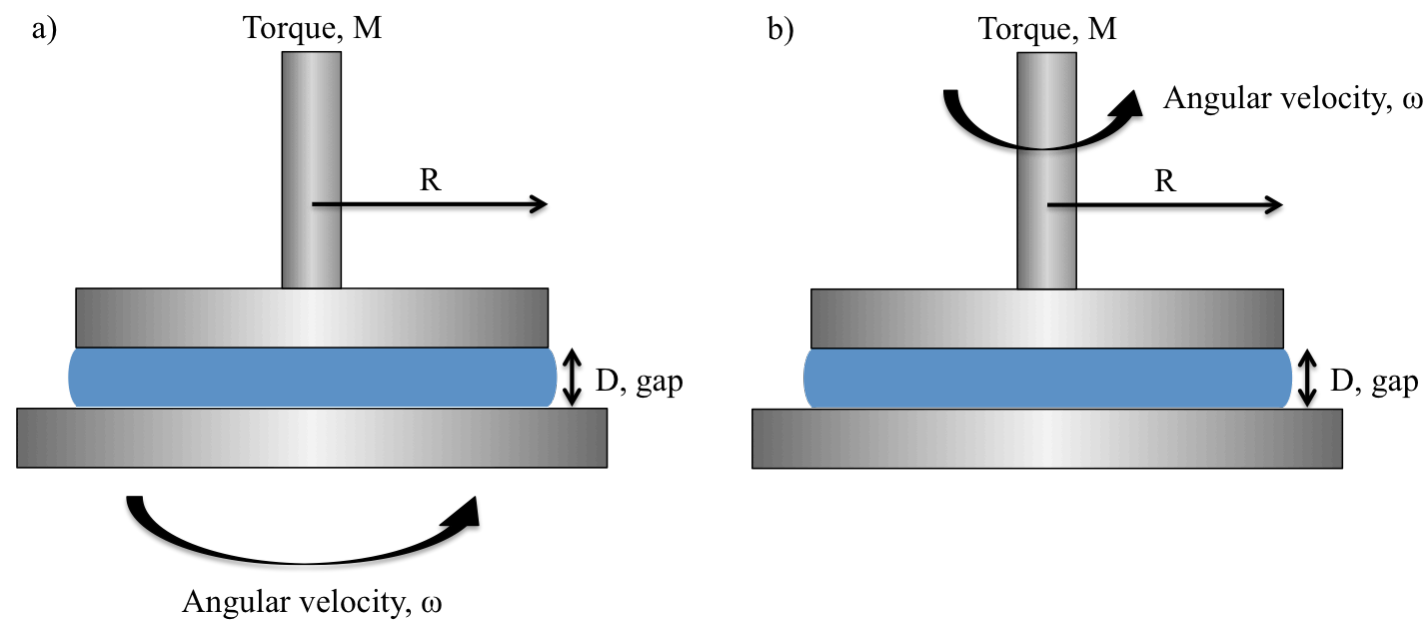

Figure 3.3 The parallel plate measuring system for (a) controlled strain rheometer and (b) controlled stress rheometer

\subsubsection{Cone and plate}

The cone and plate geometry consists of an upper cone that is truncated at the centre and a lower plate. There is no contact between the plates. However, the gap size is very small and therefore usually single-phase homogeneous samples or samples with submicron particles are measured with cone and plate geometries. In general, test materials, which contain particles, are not suitable for cone and plate geometries, because the particles can migrate to the apex of the cone and can get stuck in the truncation area. This will lead to artefacts or "bad" data.

Cone and plate geometries produce homogeneous shear rate as a function of radial position. This simplifies the equations used to determine stress and strain. Therefore, it is preferable to use cone and plate geometry when measurements are conducted in the nonlinear viscoelastic region or in case of time dependent materials. However, due to the very small size of the gap the effect of thermal expansion, if operating over a range of temperatures, can cause significant errors in the rheological measurements (Mezger 2015). 
Figure 3.4 illustrates the schematic of a cone and plate system.
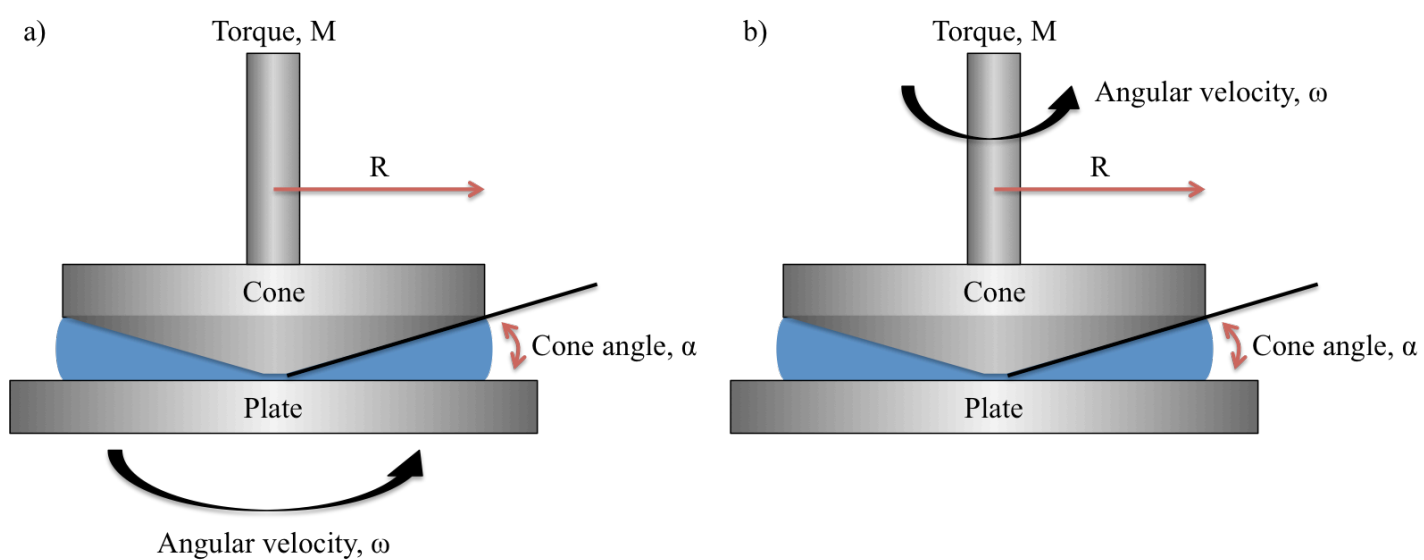

Figure 3.4 The cone and plate measuring system for (a) strain control rheometer and (b) stress control rheometer

Shear rate:

$$
\dot{\gamma}=F_{\dot{\gamma}} \omega=\frac{1}{\tan \alpha} \omega \stackrel{\text { at small angles }}{\longrightarrow} \dot{\gamma} \approx \frac{1}{\alpha} \omega
$$

Shear stress:

$$
\sigma=F_{\sigma} M=\frac{3}{2 \pi R^{3}} M
$$

\subsubsection{Narrow gap double wall concentric cylinder}

Narrow gap double wall concentric cylinder geometries are useful for studying very low viscosity samples that cannot be tested using cone and plate or parallel plate geometries. This type of geometry consists of two parts, the lower part is the cup, where the sample can be loaded, and then the upper geometry, the bob, is lowered into the sample (Figure 3.5). During rheological measurements, the sample is sheared between the wall of the cup and the upper geometry. One of the greatest advantages of the double wall concentric cylinder geometry is that in comparison to parallel plates or cone and plate geometries, it has a much larger surface area. For this reason, this geometry is often used for weakly structured or strain sensitive materials, such as gelling systems, thus maximising the torque generated (Mezger 2015). 


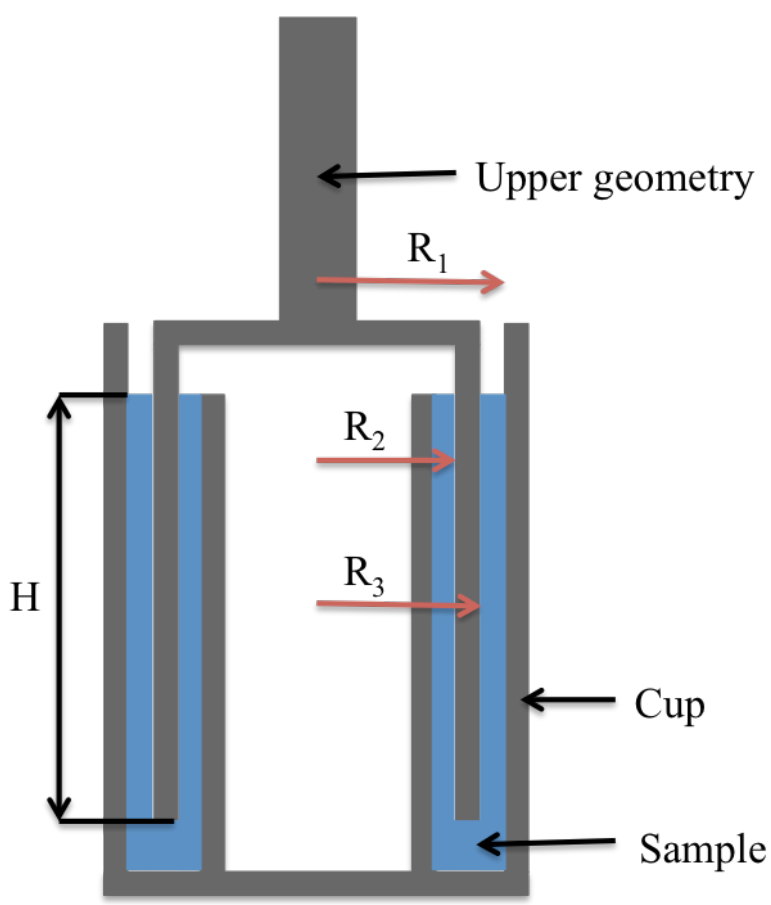

Figure 3.5 Schematic representation of the cross-section of a narrow gap double wall concentric cylinder geometry

$$
\begin{gathered}
F_{\sigma}=\frac{R_{1}^{2}-R_{2}^{2}}{4 \pi H R_{2}^{2}\left(R_{1}^{2}+R_{2}^{2}\right)} \\
F_{\dot{\gamma}}=\frac{R_{2}^{2}+R_{1}^{2}}{R_{2}^{2}-R_{1}^{2}}
\end{gathered}
$$

\subsubsection{Sample loading}

The appropriate sample loading procedure is crucial in a rheological experiment, since it affects the results. Under filling will lead to a smaller measured torque, while over filling the sample will cause additional measured torque due to the excess material dragged along the edge of the geometry. To precisely fill the gap, it is often necessary to dispense a known amount of sample using a pipette or syringe. The shearing process associated with transport of material first from the container in to the syringe and subsequently in to the rheometer geometry might disrupt (breakdown) the structure of the sample. 
Several types of mistakes can appear due to the incorrect sample loading, such as over filling or under filling (Figure 3.6) (Mezger 2015). The magnitude of the error primarily depends on the investigated sample, however usually overloading of the sample causes smaller error than under filling.
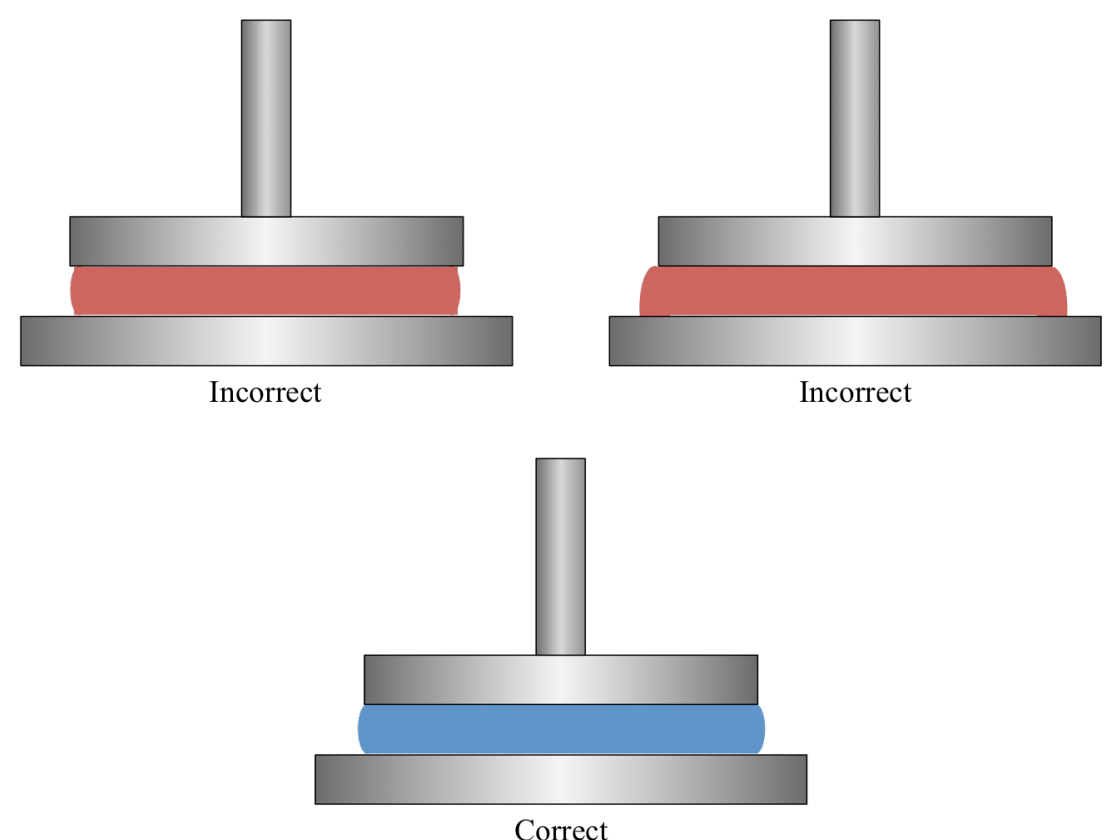

Figure 3.6 Examples for incorrect and correct sample loading

When the gap is overloaded, some of the excess material might interact with the outer edge of the geometry. This phenomenon is unlikely in the case of low viscosity materials; therefore the measurement error caused by overfilling is reduced.

The diameter of sample is assumed to be equal to that of the geometry, which is used to calculate the rheological properties. When the gap is under-filled, the diameter of the sample is not equal to that of the geometry therefore large errors can occur as the sample size is overestimated by the software. 


\subsubsection{Experimental considerations and limitations in rheometry}

\subsubsection{Linear viscoelastic range}

Small Amplitude Oscillatory Shear experiments are, by definition, conducted within the linear viscoelastic range of the material of interest. These experiments allow the study of the material's evolving rheological properties without disrupting its structure. Higher harmonic contributions in the measured stress or strain waveform indicate nonlinearities. Therefore, by monitoring the third harmonic contribution, it can be verified that the measurement is conducted within the LVR (Hawkins et al. 2010).

\subsubsection{Sample mutation}

During gelation, the rheological properties of a material are constantly changing i.e. the sample is transient. The characteristic time for change in the material is called mutation time, $\lambda_{m u}$ (Winter 2002).

$$
\lambda_{m u}=\left(\frac{1}{G^{\prime}}\left|\frac{\partial G^{\prime}}{\partial t}\right|\right)^{-1}
$$

The mutation time is the time required for the moduli to increase by factor $e$ or decrease by factor $1 / e$ (Winter \& Mours 1997). In this case the formula contains the storage modulus, since the change in $G^{\prime}$ is greater than the change in $G^{\prime \prime}$ during gelation (Winter 2002). Apart from the rate of gelling, the mutation time is also dependent on the measuring frequency (Scanlan \& Winter 1991).

$$
\lambda_{m u} \sim \omega^{\kappa}
$$

The value of $\kappa$ is typically 0.2 for most gelling systems. The ratio of experimental time, which is the time taken during measurement for which $G^{\prime}$ is calculated, to mutation time defines the mutation number, $N_{m u}$. 


$$
N_{m u}=\frac{\Delta t}{\lambda_{m u}}
$$

If the value of the mutation number is below $10 \%$, the collected data points are acceptable (Winter 2002).

Another criteria for considering sample mutation during gelation was proposed by Hawkins et al. (Hawkins et al. 2010). The gelation can be monitored in a Gel Point (GP) measurement by the application of Small Amplitude Oscillatory Shear, and the GP can be captured as the sample is subjected to a sinusoidal stress waveform at different frequencies. A certain length of time, $t_{\exp }$ (experiment time) is required to apply one frequency sweep:

$$
t_{\text {exp }}=\sum_{i=1}^{i}\left(t_{c}+\frac{1}{f_{i}}\right)
$$

Where $t_{c}$ is the conditioning time and $f_{i}$ is the $i$ th value of frequency of oscillation of the measurement in $\mathrm{Hz}$.

If the ratio of $t_{\text {exp }}$ to the gel time, $t_{G P}$, is below 0.1 , the value of stress relaxation exponent, $\alpha$, measured at the GP is constant. As $t_{\text {exp }} / t_{G P}$ increases above 0.1 , the value of $\alpha$ deviates from its expected and widely reported values. This is due to the increased experiment time that causes errors during the process of interpolating between discrete frequency data points. Therefore, $t_{G P}$ must be at least 10 times larger than $t_{\text {exp }}$ in order to capture an accurate GP (Hawkins et al. 2010).

\subsubsection{Inertia considerations}

In rheological experiments, both instrument and fluid inertia should be carefully considered. When the load is measured at a moving boundary with unsteady motions, such as during acceleration, instrument inertia can cause experimental errors, as the acceleration of the instrument might also contribute to the load. Instrument inertia should be considered when measuring very soft materials, such as biomaterials, in 
oscillatory shear tests conducted at high frequency, or in short time creep data in which case the coupling of inertial and elastic forces can lead to ringing effects. In order to eliminate the effect of instrument inertia, the component torque from the instrument has to be smaller than the component material torque (Ewoldt et al. 2015). I.e.:

$$
T_{\text {material }}>T_{\text {inertia }}
$$

Therefore,

$$
\begin{gathered}
\frac{G \gamma_{0}}{F_{\tau}}>I \theta_{0} \omega^{2} \\
G>\frac{I F_{\tau}}{F_{\gamma}} \omega^{2}
\end{gathered}
$$

Where $G$ represents either the storage, $G^{\prime}$, or loss, $G^{\prime \prime}$, moduli. A raw phase difference between stress and strain waveforms exceeding $90^{\circ}$ indicates that the waveforms are polluted with inertia. The effects of instrument inertia can be corrected by calibrating the rotational inertia of the rheometer, and subtracting the "instrument inertia torque" from the total torque signal.

Apart from instrument inertia, sample inertia can affect a rheological experiment, which is caused by momentum diffusion, viscoelastic waves, and secondary flows. This is commonly seen in measurement of weakly structured or low viscosity materials. The presence of sample inertia violates the homogeneous simple shear strain assumption. Homogeneous shear strain can be sustained if the wavelength, $l$, of the propagating wave is much larger than the geometry gap, $D$ (Ewoldt et al. 2015).

$$
l \gg D
$$

The expression for the wavelength, $l$, shows that the wavelength decreases with increasing frequency, as follows, 


$$
l=\frac{1}{\cos (\delta / 2)}\left(\frac{\left|G^{*}\right|}{\rho}\right)^{1 / 2} \frac{2 \pi}{\omega}
$$

Therefore, wave propagation is likely to cause sample inertia errors at high frequencies and short time scales, especially in the case of soft gels with small modulus value.

Another type of sample inertia arises from the presence of secondary flows that are superimposed on the primary simple shear flow at high frequencies. This type of sample inertia could occur in concentric cylinder geometries with a moving inner cylinder, as well as in cone and plate or parallel plate geometries. The presence of secondary flows is perceived by the rheometer as additional torque. Selecting a smaller gap size might help avoiding this artefact. However, in the case of low viscosity fluids, secondary flows set the experimental limit in the region of high shear rates (Ewoldt et al. 2015).

\subsubsection{Temperature control}

During flow process, all materials have flow resistance as a result of the internal friction caused by the molecules and other elements of the material. Due to the internal friction during the flow process, in particular at high shear rates, some of the work done can dissipate as heat which leads to increase in the temperature a phenomenon known as viscous-shear heating (Mezger 2015). Viscous-shear heating makes maintaining the measurement temperature at a constant value challenging. The rheological properties of a material are very sensitive to the changes in temperature; therefore viscous heating can cause significant errors. Measurements at very high shear rates should be as short as possible, with fewer measurement points. In addition, appropriate temperature control is crucial in rheological experiments which can be achieved by a Peltier system. 


\subsubsection{Wall slip and edge effects}

In the rheological measurements a 'no slip' condition is assumed. This means that the sample closest to the surface of the geometry moves with the velocity of that surface. However, velocity difference can occur, which leads to inaccurate experimental results. In the case of multiphase systems, apparent wall slip or lubrication might occur as the dispersed particles move away from the wall, due to steric, hydrodynamic, viscoelastic or chemical forces. This phenomenon is dependent on the particle size, or floc size in case of flocculated suspensions. The floc size is shear rate dependent, larger flocs form at lower shear rates; therefore wall slip occurs most often at low shear rates. Slip can be prevented by roughening or profiling the surface of the geometries. If measurements are conducted with different gap sizes but at the same frequency and strain, then the consistence in the produced dynamic moduli values yields the absence of slip. Alternatively, vane geometry can be used, which is beneficial especially for testing fibrous samples, as any random orientation of the fibres near the wall can be avoided (Barnes 2000).

Edge effects can occur for several reasons depending on the type of geometry used. In the case of cone and plate or parallel plate geometries, the conditions at the edge of the geometry are crucial, because the stress close to the edge contribute significantly to the measured torque (Barnes 2000). Edge effects can be exaggerated when the outer surface of the sample is in contact with the surrounding atmosphere. This can result in evaporation of the material or a 'skin' layer at the edge, which is different in composition to the rest of the sample material. There are different ways to prevent evaporation, such as using a vapour hood or in the case of water-based materials the test sample can be surrounded with low viscosity silicon oil. Edge effect also can occur due to surface tension, but these effects are considered negligible when a cone with an angle less than 4 degrees is used.

\subsection{Scanning Electron Microscope (SEM)}

A scanning electron microscope (SEM) is type of electron microscope that provides valuable information on the surface topography and composition of the examined sample, with resolutions lower than $1 \mathrm{~nm}$ (Yoshida et al. 2016; Henning \& Adhikari 
2017). Several previous studies have used SEM images of fibrin gels and whole blood clots in order to investigate the structure of the formed network (Veklich et al. 1998; Zhao et al. 2008; Brown et al. 2009; Janmey et al. 2009; Weisel \& Litvinov 2013b; Lawrence et al. 2014; Badiei et al. 2015; Litvinov \& Weisel 2017). In this study SEM is used to image fibrin clots that had been previously subjected to different shear stresses between the rheometer plates.

The basic operation principle of the SEM is that an electron beam is focused on to, and scans the surface of the test specimen (Yoshida et al. 2016; Henning \& Adhikari 2017). The electrons interact with the atoms in the specimen, and as a result of this interaction a variety of signals are created, such as secondary electrons, backscattered electrons, transmitted electrons, specimen current (i.e. absorbed current), and characteristic X-rays and light. The X-rays that are created during the interaction do not destroy the sample; therefore it is a non-destructive measurement, allowing repeated imaging of the same sample. The most commonly used signals for imaging are the secondary electrons, which are useful for obtaining morphological and topological characteristics of the sample, and back-scattered electrons, which show contrasts in composition in multiphase samples. The samples can be imaged in different experimental conditions, such as in high vacuum in convectional SEM, in low vacuum, in wet conditions, or in variable pressure, and in a wide range of temperatures, from cryogenic to elevated temperatures.

Herein, the purpose of using SEM is to provide highly complementary imaging studies of clots formed with different mechanical and microstructural properties and to explore the mechanisms at a macroscopic level that may lead to different nonlinear viscoelastic behaviour. 


\section{LAOStrain characterisation of fibrin gels}

\subsection{Aims and objectives}

The purpose of this chapter is to investigate the role of structure in the eventual nonlinear viscoelastic properties of mature (i.e. fully formed) fibrin clots by performing Large Amplitude Oscillatory Shear Strain (LAOStrain) experiments. Furthermore, it is aimed to investigate the propensity of clots to embolise by measuring the nonlinear rheological response to increasing levels of shear stresses, including those commensurate with the levels of shear stresses encountered in blood vessels in vivo, up to the point of fracture. To this end, a study of the effect of loading history on the mechanical and structural properties of mature fibrin clots was explored using a rheological protocol that involved the application of LAOStrain followed by linear viscoelastic measurements of the clots.

\subsection{Materials}

Fibrin gels with a final fibrinogen concentration of $4 \mathrm{mg} / \mathrm{ml}$ were used in the measurements as a model system for blood clotting (Table 4.1). Purified, plasminogen depleted human fibrinogen at least $95 \%$ clottable, and human thrombin were obtained from Enzyme Research Laboratories, UK. The fibrinogen was made up to stock solution of $44.54 \mathrm{mg} / \mathrm{ml}$ by the addition of Tris-buffered saline, TBS (20 mM Tris, pH 7.4 and $0.9 \% \mathrm{NaCl}$, Sigma Aldrich, UK), and left to fully dissolve in a water bath at $37^{\circ} \mathrm{C}$. The thrombin was dissolved in distilled water and made up to a stock solution of $500 \mathrm{NIH}$ Units $/ \mathrm{ml}$. This stock solution was decanted into small eppendorf tubes and frozen at $-80{ }^{\circ} \mathrm{C}$ until required. Tween 20 (J. T. Baker, USA) was added to TBS to make up a $1 \mathrm{wt} \%$ stock solution of TBS-Tween 20, which was stored at $4{ }^{\circ} \mathrm{C} .50 \mathrm{mM}$ Factor XIII inhibitor (Zedira GmbH, Germany) stock solution was also prepared by dissolving the powder in Phosphate Buffered Saline, PBS (pH 7.4, Gibco by Life Technologies, UK). The FXIII inhibitor stock solution was allocated into small vials and stored at $-20^{\circ} \mathrm{C}$. 
The appropriate volume of TBS-Tween 20, normal TBS, fibrinogen and $\mathrm{CaCl}_{2}$ were mixed together to make a final concentration of $4 \mathrm{mg} / \mathrm{ml}$ Fibrinogen, 0.1 wt \% Tween 20 and $0.005 \mathrm{M} \mathrm{CaCl}_{2}$. To study the effect of FXIII mediated crosslinking FXIII inhibitor was added to the mixture at a $1 \mathrm{mM}$ final concentration prior to the addition of $\mathrm{CaCl}_{2}$. Clotting was initiated by the addition of the required thrombin volume to produce a final thrombin concentration in the range of 0.02 to $0.1 \mathrm{NIH}$ Unit/ml. After appropriate mixing using a pipette, the sample was transferred immediately to the rheometer plates.

Table 4.1 Components of the fibrin gel

\begin{tabular}{|c|c|c|}
\hline Component & Bulk concentration & Final concentration \\
\hline Tween20 & $1 \mathrm{wt} \%$ & $0.1 \mathrm{wt} \%$ \\
\hline Normal TBS & & $4 \mathrm{mg} / \mathrm{ml}$ \\
\hline Fibrinogen & $44.54 \mathrm{mg} / \mathrm{ml}$ & 0 or $1 \mathrm{mM}$ \\
\hline Factor XIII inhibitor & $50 \mathrm{mM}$ & $0.005 \mathrm{M}$ \\
\hline CaCl2 & $1 \mathrm{M}$ & $0.02-0.1 \mathrm{NIH} / \mathrm{ml}$ \\
\hline Thrombin & $500 \mathrm{NIH} / \mathrm{ml}$ & \\
\hline
\end{tabular}

\subsection{Methods}

\subsubsection{LAOStrain measurements of fibrin gels}

Rheological measurements were conducted using ARES-G2 controlled strain rheometer (TA Instruments, UK) fitted with a cone and plate (50 $\mathrm{mm}$ cone, 0.04 radian) measuring system. The required temperature was maintained at $24{ }^{\circ} \mathrm{C}$ using a Peltier temperature control system in order to control the rate of clotting. Before the start of each test, the samples were surrounded with small amount of low viscosity silicon oil (Fluid 10, $9.7 \mathrm{cP}$, Viscosity Standard, Brookfield, U.S.A.) in order to prevent sample evaporation. The fibrinogen concentration was within the physiologically relevant range $(1.5$ to $4 \mathrm{mg} / \mathrm{ml})$, while the thrombin concentration and temperature was within a sub-physiological range to reduce the rate of gelation in order to adhere to rheological constrains such as mutational effects. The LAOStrain properties of fully formed fibrin clots were characterised as a model 
system for blood. Fibrin clots are considered fully formed, i.e. "mature" clots, once their viscoelastic properties suggest no further microstructural changes (i.e. measured phase angle is constant or storage modulus, $G^{\prime}$, reaches a plateau), which occurs after a period of 40 times the gel time, $t_{G P}$ (Curtis et al. 2013). Clots with different values of $d_{f}$ were manipulated by adding a range of thrombin concentrations $(0.02-0.1$ $\mathrm{NIH} / \mathrm{ml})$ to a fixed amount of fibrinogen $(4 \mathrm{mg} / \mathrm{ml})$. Uncrosslinked clots were formed by adding Factor XIII (FXIII) inhibitor to the model system. The evaluation of viscoelasticity, in terms of storage $\left(G^{\prime}\right)$ and loss $\left(G^{\prime \prime}\right)$ moduli, during the clot formation was monitored in a "time sweep" measurement by the application of strain waveform with a strain amplitude value (1\%) which was within the LVR as confirmed by the absence of a third harmonic response. During the process of clot formation, $G^{\prime}$ gradually increases until it reaches a plateau that indicates that the clot is fully formed. Subsequently, the fracture properties of mature fibrin clots were measured in a LAOStrain test, by applying continuous sinusoidal strain to the mature clot in a logarithmically increasing stepwise mode $(0.1-300 \%)$ up to the point of fracture, and the resulting stress is measured. Linear strain sweep was applied between 100 and $300 \%$ in order to obtain more data points in the regions of interest. In each measurement, the sample was allowed to reach a steady state as described in Section 4.4.1.

\subsubsection{Effect of strain history on the fracture properties of fibrin gels}

Experiments were conducted in order to investigate the ability of the fibrin network to adapt (by irreversible rearrangements of structure) to the imposed strain (Figure 4.1). In SAOS measurement, $G^{\prime}$ and $G^{\prime \prime}$ were monitored during the process of clot formation by the application of strain waveform with a strain amplitude (1\%) within the LVR for 40 times the gel time, until the clot reaches its 'mature' form (Stage 1). Following that, a sinusoidal strain waveform with constant strain amplitude (in a range of 1 to $120 \%$ ) was applied to the mature clot until the linear or nonlinear rheological response reached a steady state (Stage 2). Subsequently LAOStrain tests were conducted on the same clot to measure the fracture properties, by applying consecutive, increasing sinusoidal strain waveforms to the mature clot in a stepwise mode $(0.1-300 \%)$ up to the point of fracture (Stage 3$)$. 


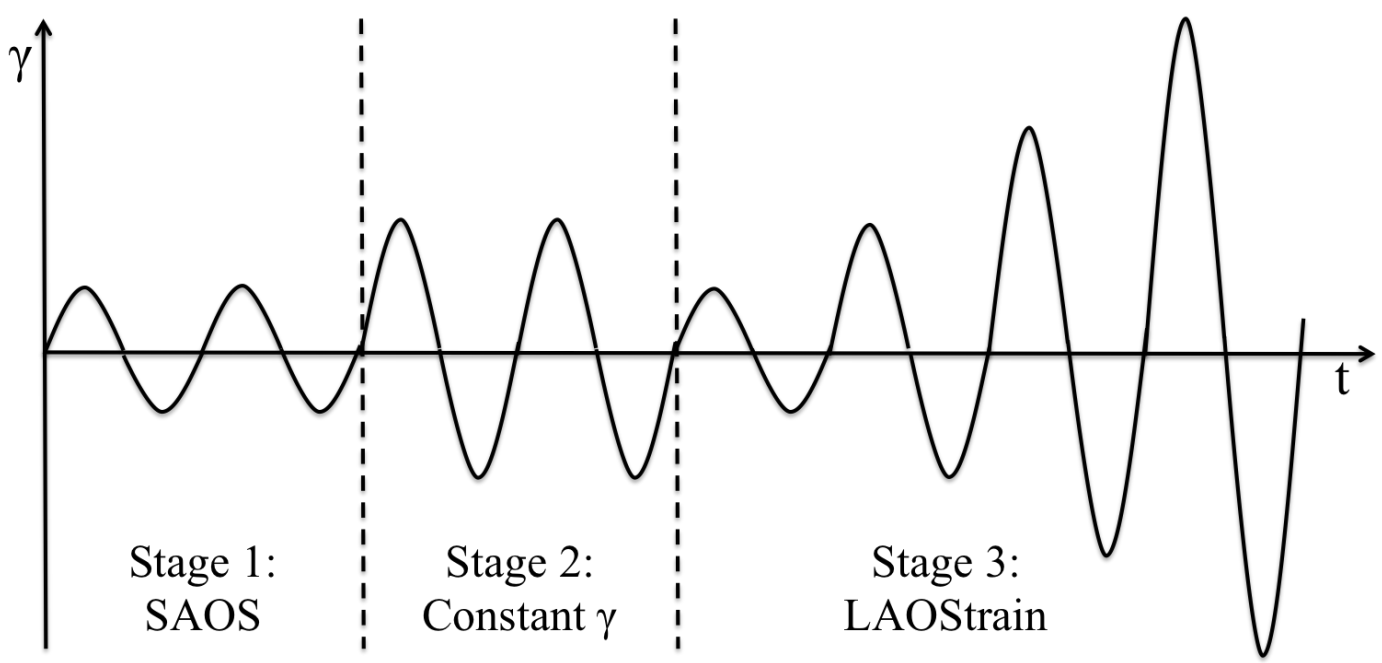

Figure 4.1 Schematic representation of the procedure for measuring the effect of strain history on the fracture properties of fibrin gels. Stage 1: Application of SAOS for monitoring the microstructural evolution of the clot. Stage 2: A sinusoidal waveform with constant strain amplitude in the range of $1 \%$ to $120 \%$ is applied to the fully formed clot. Stage 3 : Strain sweep between $0.1 \%$ to $300 \%$.

\subsubsection{Structural changes during LAOStrain}

This experimentation section was designed in order to obtain information of the ability of fibrin clots to adapt to the imposed deformation, by applying one experimental procedure, which summarises all the individual tests performed in the previous chapter. LAOStrain tests were conducted on the 'mature' fibrin clot, and 1 $\%$ (linear) strain was applied between each strain amplitudes of the LAOStrain measurement. The effect of different thrombin concentrations $(0.02,0.06$ and 0.1 $\mathrm{NIH} / \mathrm{ml}$ ) was studied.

\subsection{Results}

\subsubsection{Steady state criteria in LAOS}

For the meaningful evaluation of the nonlinear data, it is assumed that the input strain waveform is perfectly sinusoidal and any nonlinearity is due to the nonsinusoidal stress response waveform, and the resulting stress is independent of the shearing direction (Hyun et al. 2011). 


$$
\sigma[-\gamma(t),-\dot{\gamma}(t)]=-\sigma[\gamma(t), \dot{\gamma}(t)]
$$

In order to satisfy this criterion large strain oscillations should be applied for several cycles until a steady state is reached before the nonlinear data collection (Giacomin \& Dealy 1993). Currently there is no standardised method which defines, when this steady state is reached, and thus a standardised criteria is required that can be applied for all materials and for all nonlinear tests. In the following, an approach utilising a method to quantify the point symmetry of the waveforms is proposed.

Figure 4.2a shows the resulting stress in two cycles of oscillation. In steady state, the nonlinear stress waveform should be exactly the same in one shearing direction (i.e. $C_{1}$ or $C_{3}$ ) as it is in the counter shearing direction (i.e. $C_{2}$ or $C_{4}$ ). By studying the absolute value of the stress waveform in Figure $4.2 \mathrm{~b}$, it can be seen that the point symmetry criteria is only fulfilled if $C_{1}=C_{2}$ and $C_{3}=C_{4}$; therefore, the standard deviation of $C_{1}$ to $C_{2}$ and $C_{3}$ to $C_{4}$ has to be zero.
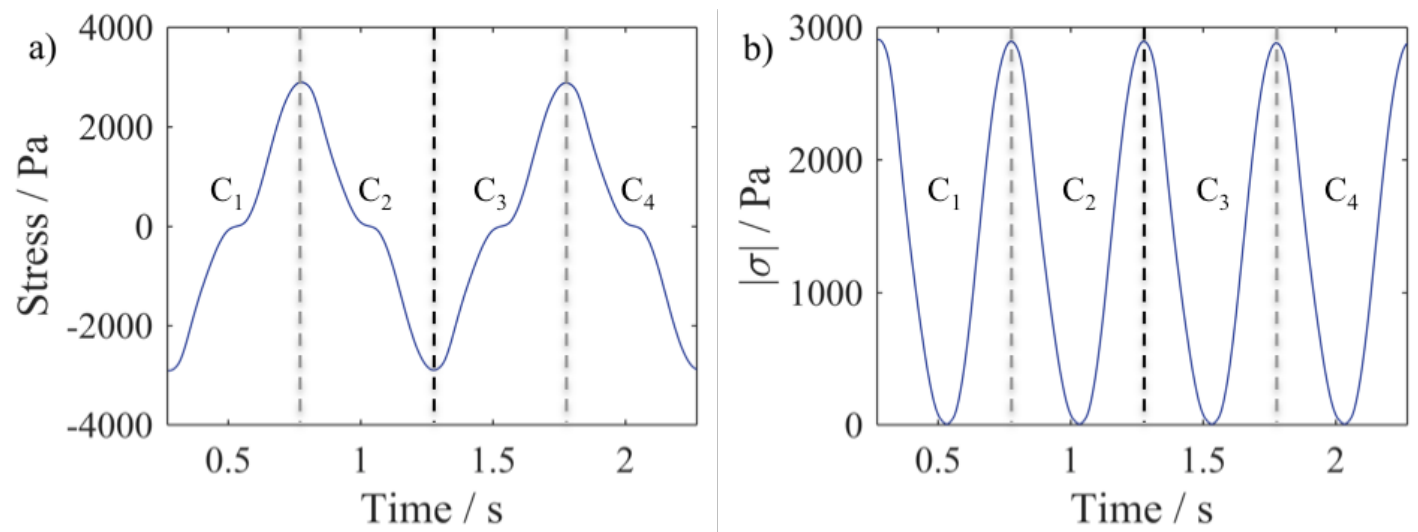

Figure 4.2 (a) Measured stress waveform as a function of time, and (b) absolute value of the stress waveform.

Each data point in $C_{1}$ was paired to its corresponding data point in $C_{2}$ (Figure 4.2b), and the standard deviation of these two points was calculated. Following that the standard deviations calculated for each data pair were averaged for each oscillation cycle. Results in Figure 4.3 show that the standard deviation of each cycle in case of an LVR data (1\% strain) is around zero. Increasing levels of strains resulted in increasing standard deviations, and more cycles of oscillation required for reaching steady state. The standard deviation stayed below $1 \mathrm{~Pa}$ in the case of $40 \%$ strain, whist a few cycles of oscillations were needed for $60 \%$ applied strain for its standard 
deviation values to level off. In the case of an even higher applied strain $(100 \%)$ the calculated standard deviations levelled off after approximately 380 cycles of oscillations.

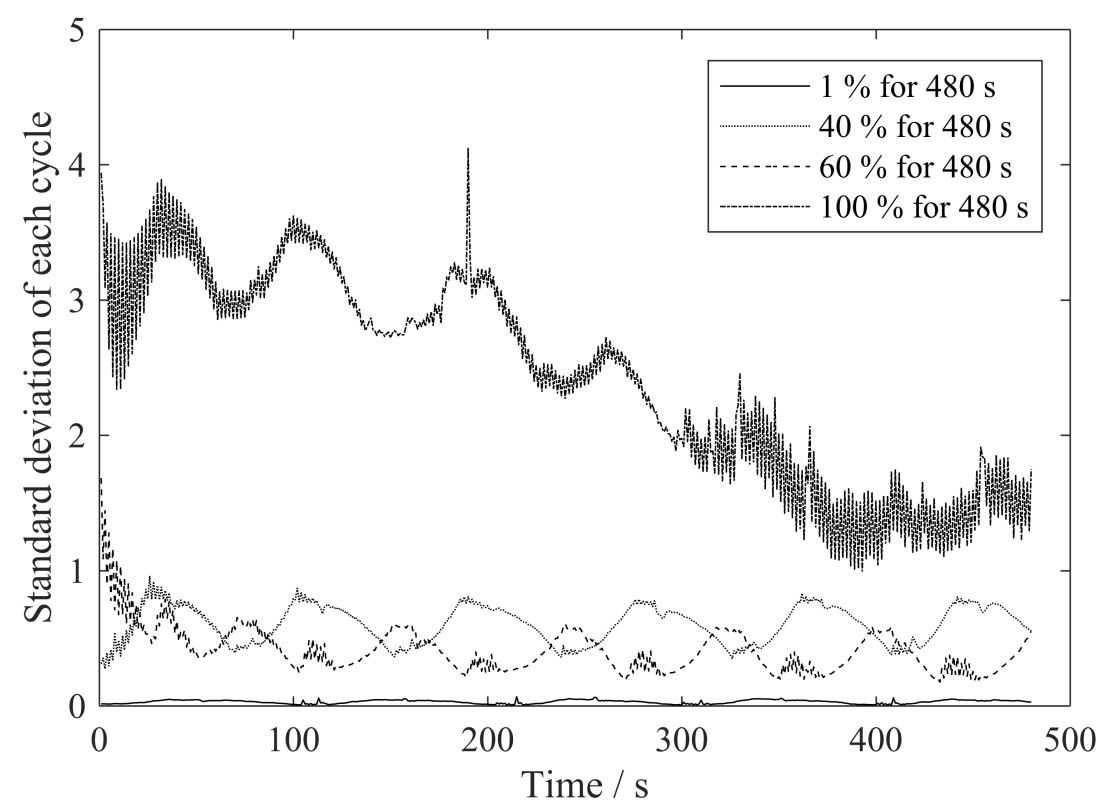

Figure 4.3 Standard deviations calculated for oscillatory cycles as a function of time.

It is important to note, that due to the strain stiffening characteristic of the fibrin gel, as the applied strain amplitude is increasing, the resulting stress amplitude is also increasing (Figure 4.4). This should be considered when assessing the calculated standard deviation values. 


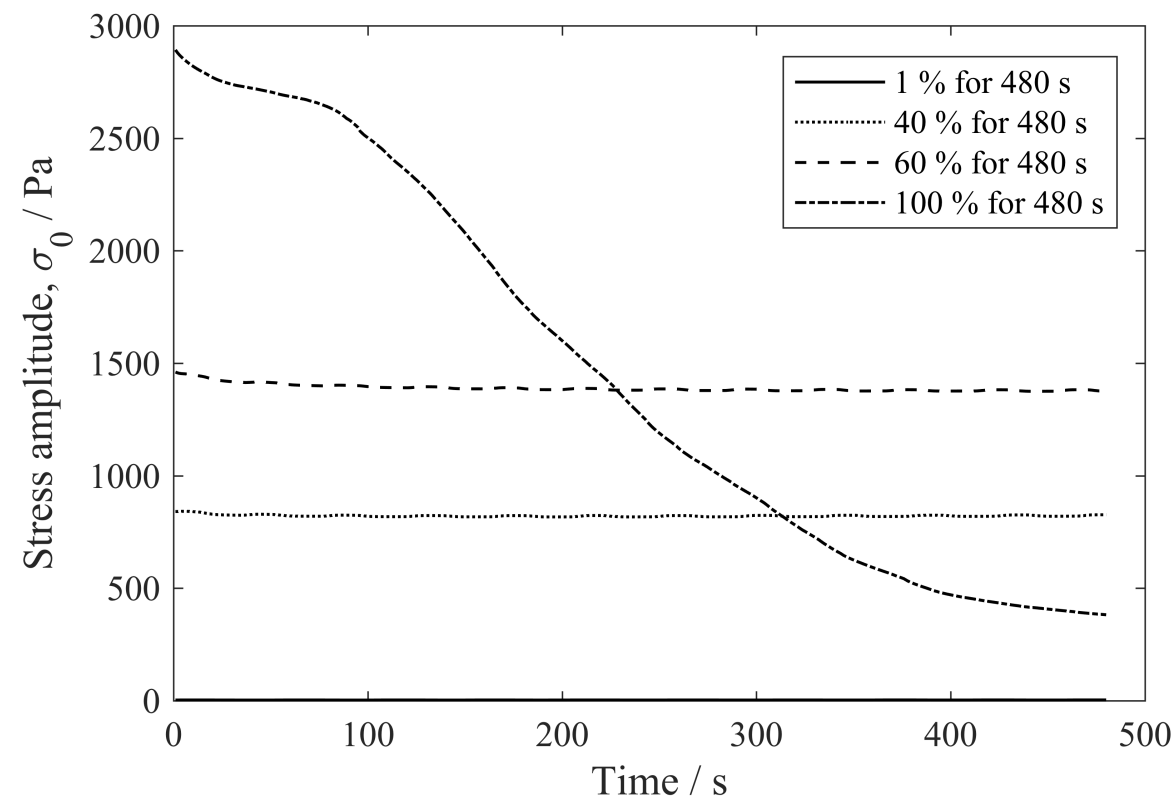

Figure 4.4 The measured stress amplitudes as a function of time in the case of various applied strain levels from $1 \%$ to $100 \%$.

In order to account for the effect of the increasing stress amplitudes with increasing applied strain, the absolute stress differences between consecutive stress amplitudes were calculated (Figure 4.5), as well as the changes were calculated in terms of percentages, which represents the difference relative to the resulting stress levels (Figure 4.6).

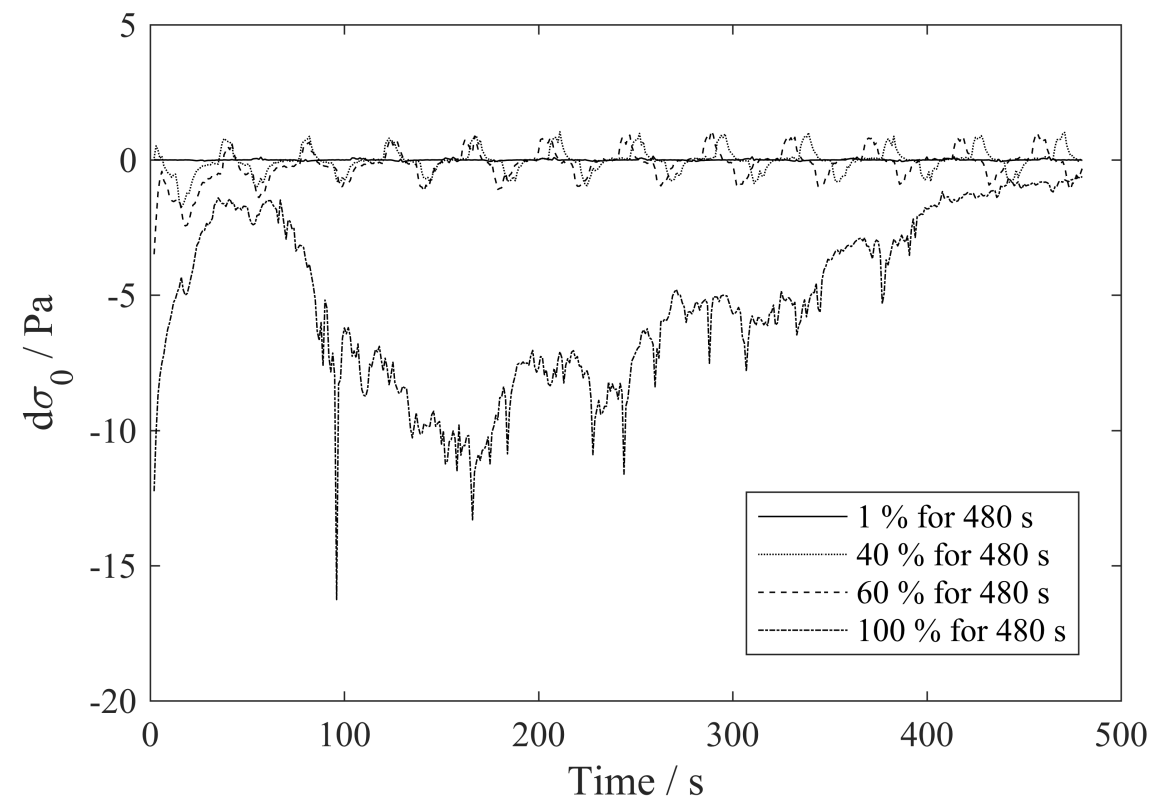

Figure 4.5 Stress difference between consecutive stress amplitudes as a function of time. 


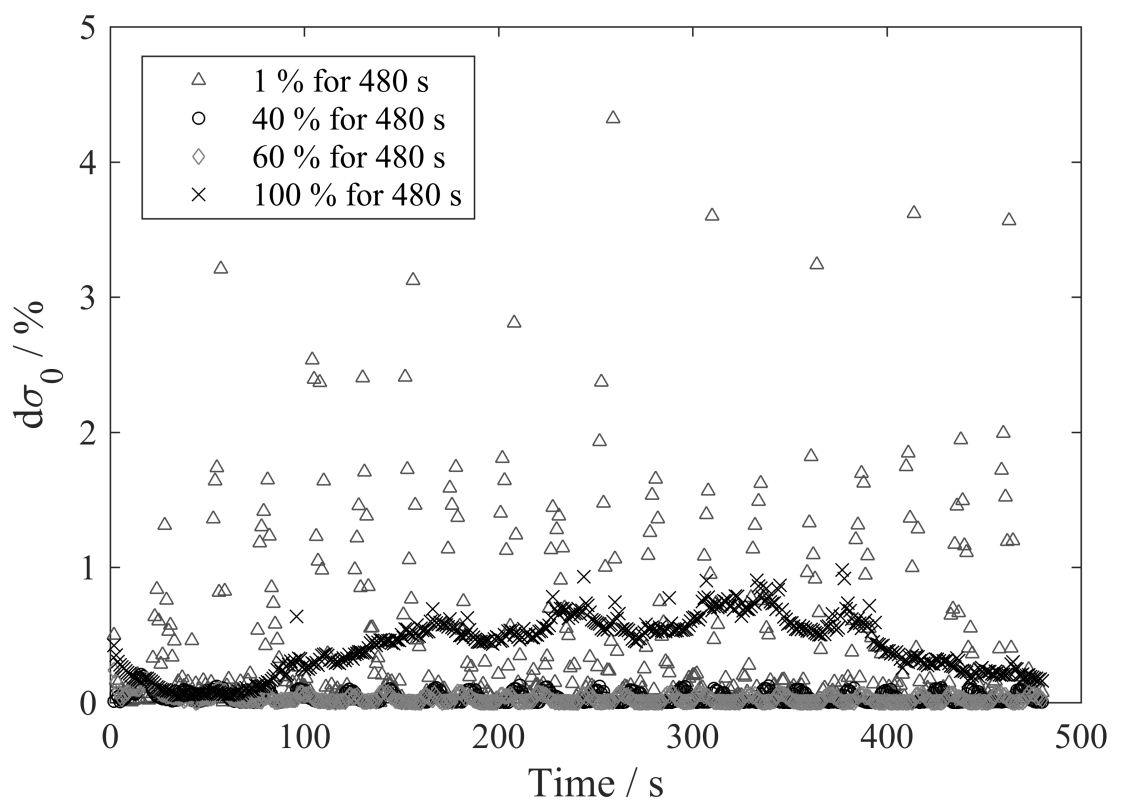

Figure 4.6 Stress difference between consecutive stress amplitudes in terms of percentages.

Based on the calculated standard deviations of each oscillation cycle (Figure 4.3), and the percentage difference in measured stress amplitudes (Figure 4.6), the following criteria was set for defining steady state in LAOS. The materials response to the applied large strain or large stress is considered to reach a steady state once the difference in stress or strain response between consecutive waveforms is less that 0.2 $\%$ or less than $2 \%$ in the case of SAOS. This criterion ensures that point symmetry is present in the Lissajous-Bowditch curves obtained from the LAOS tests and capturing the irreversible damage following relaxation of the samples probed by SAOS. 


\subsubsection{LAOStrain measurement of fibrin gels}

The nonlinear behaviour, in terms of fracture stress, nonlinear moduli and stiffening ratio, of mature fibrin clots formed from $4 \mathrm{mg} / \mathrm{ml}$ fibrinogen and various thrombin concentrations $(0.02,0.06$ and $0.1 \mathrm{NIH} / \mathrm{ml})$ were measured. The fracture stress was identified using elastic Lissajous-Bowditch plots, which graphically represent the total stress as a function of applied strain. The fracture stress is the maximum measured stress during the LAOStrain experiment. As the strain amplitude is increasing, the enclosed area within the stress versus strain "loop" represented in the Lissajous-Bowditch plots increased. This loop or enclosed area represents the viscous energy dissipation within a cycle of oscillation (Ewoldt et al. 2010; Sun et al. 2015). Therefore, as the applied strain is increasing the fibrin clot is becoming more and more viscous. The maximum stress values within the Lissajous-Bowditch plots increase up to the point of fracture (Figure 4.7d), and then decrease following increases in applied strain (Figure 4.7e). The shape of the Lissajous-Bowditch plots deviates from an ellipse, indicating nonlinear viscoelastic behaviour, and also show the remarkable strain stiffening behaviour of the fibrin clots, as the slope of the curves increased with increased strain within a given steady state cycle (Figure 4.7). 


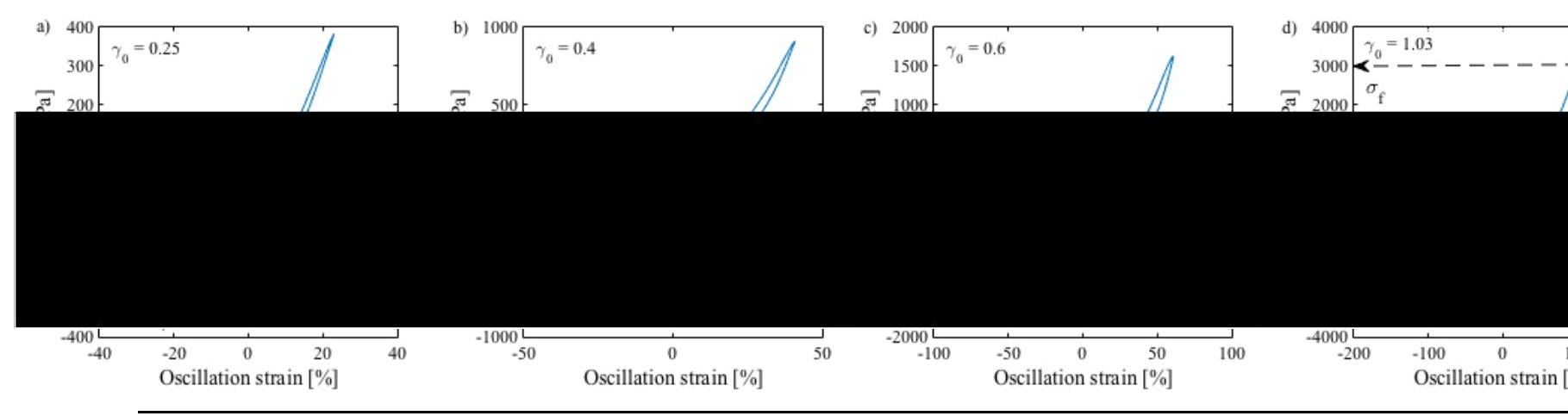

Increasing strain amplitude

Figure 4.7 Lissajous-Bowditch plots (the total stress and the corresponding elastic stress contribution as a function of strain at different strain fibrinogen and $0.1 \mathrm{NIH} / \mathrm{ml}$ thrombin. As the strain amplitude is increasing (from (a) to (e)), the enclosed area within the loop (represents the vis al. 2010; Sun et al. 2015)) is increasing, and the clot is becoming more viscous. The maximum stress values increases until the point of fracture stress and strain values at the point of fracture, fracture stress $\left(\sigma_{\mathrm{f}}\right)$ and fracture strain $\left(\gamma_{\mathrm{f}}\right)$ respectively. 
It was expected that clots formed with different levels of thrombin concentrations would withstand different levels of maximum stresses, as the varying thrombin levels result in different $d_{f}$ values, hence different structures (Curtis et al. 2013). However, in the case of both crosslinked (i.e. presence of FXIII) and uncrosslinked (i.e. absence of FXIII) fibrin clots the fracture stress was not affected by the level of thrombin present in the clot (Figure 4.8). A lower level of fracture stress was measured for the clots formed without FXIII (i.e. uncrosslinked clots) compared to the clots formed with FXIII (i.e. crosslinked clots), which was expected because FXIII initiates the formation of covalent bonds (crosslinks) between the fibrin fibres, which stabilise the structure of the fibrin clot (Chernysh et al. 2012).

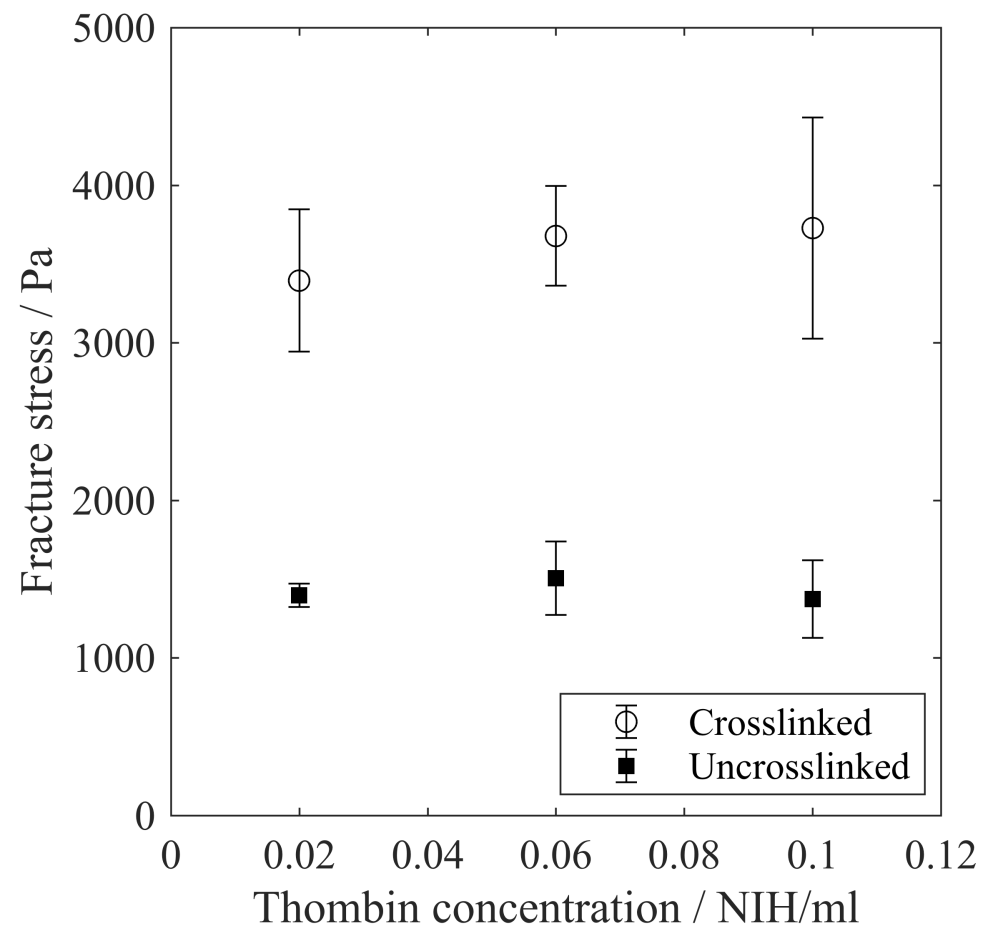

Figure 4.8 Fracture stress as a function of thrombin concentration in case of crosslinked and uncrosslinked fibrin clots

Clot formation, in terms of the evolution of linear viscoelastic properties, was monitored in a SAOS step. In order to account for both elastic and viscous behaviour, the strength of the fully formed clot is described by the measured maximum complex modulus, $G_{\max }^{*}$, value. In order to investigate the effect of the deviation of the $G_{\text {max }}^{*}$ of different samples, the fracture stress of each sample is plotted as a function of $G_{\max }^{*}$ in Figure 4.9. In the case of the crosslinked fibrin clots 
(when FXIII inhibitor was not added), and a maximum complex modulus less than $500 \mathrm{~Pa}$, the fracture stress is only slightly affected by the maximum clot strength. Above $G_{\max }^{*}=500 \mathrm{~Pa}$, the fracture stress is decreasing as $G_{\max }^{*}$ is increasing. In experiments whereby FXIII inhibitor was added in order to form uncrosslinked fibrin clots, the measured $G_{\max }^{*}$ values are similar to those measured for the crosslinked fibrin clots. However the fracture stresses are lower, and level of $G_{\max }^{*}$ does not affect the fracture stress.

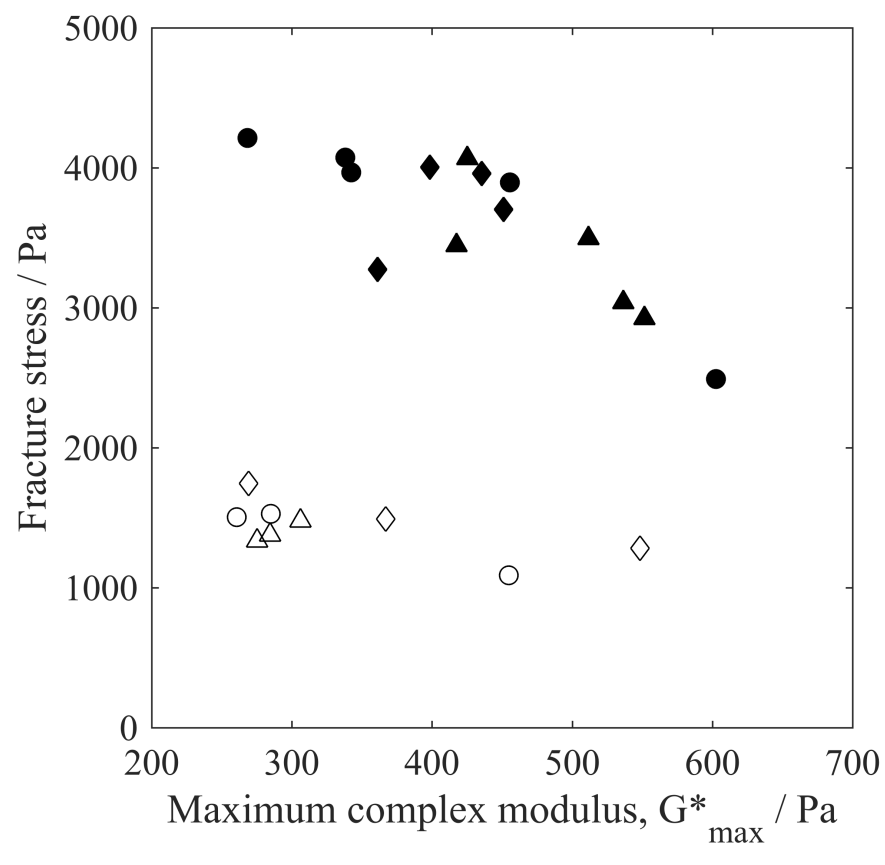

\begin{tabular}{|cl|}
\hline$\bullet$ & $0.1 \mathrm{NIH} / \mathrm{ml}$ Thrombin \\
$\bullet$ & $0.06 \mathrm{NIH} / \mathrm{ml}$ Thrombin \\
$\triangleright$ & $0.02 \mathrm{NIH} / \mathrm{ml}$ Thrombin \\
$\diamond$ & $0.1 \mathrm{NIH} / \mathrm{ml}$ Thrombin + FXIII inhibitor \\
$\triangle$ & $0.06 \mathrm{NIH} / \mathrm{ml}$ Thrombin + FXIII inhibitor \\
$\triangle$ & $0.02 \mathrm{NIH} / \mathrm{ml}$ Thrombin + FXIII inhibitor \\
\hline
\end{tabular}

Figure 4.9 Effect of the maximum complex modulus measured in the SAOS step, on the fracture stress of the fully formed fibrin clots

Frequency sweeps (between 0.1 to $10 \mathrm{~Hz}$ ) were performed by applying oscillatory strain waveforms, at a strain of $1 \%$, within the LVR of the material, in order to study the structure of the fully formed and broken fibrin clots. The results show that the phase angle measured for the fully formed clots before they were fractured is very low, suggesting highly elastic behaviour (Figure 4.10). As expected, the phase angle is lowest for the highest thrombin concentration, as the high thrombin concentration 
creates a more dense, compact structure. Increased values of phase angle, i.e. increased viscous contributions were measured for the clots that had been previously fractured in a LAOS experiment.

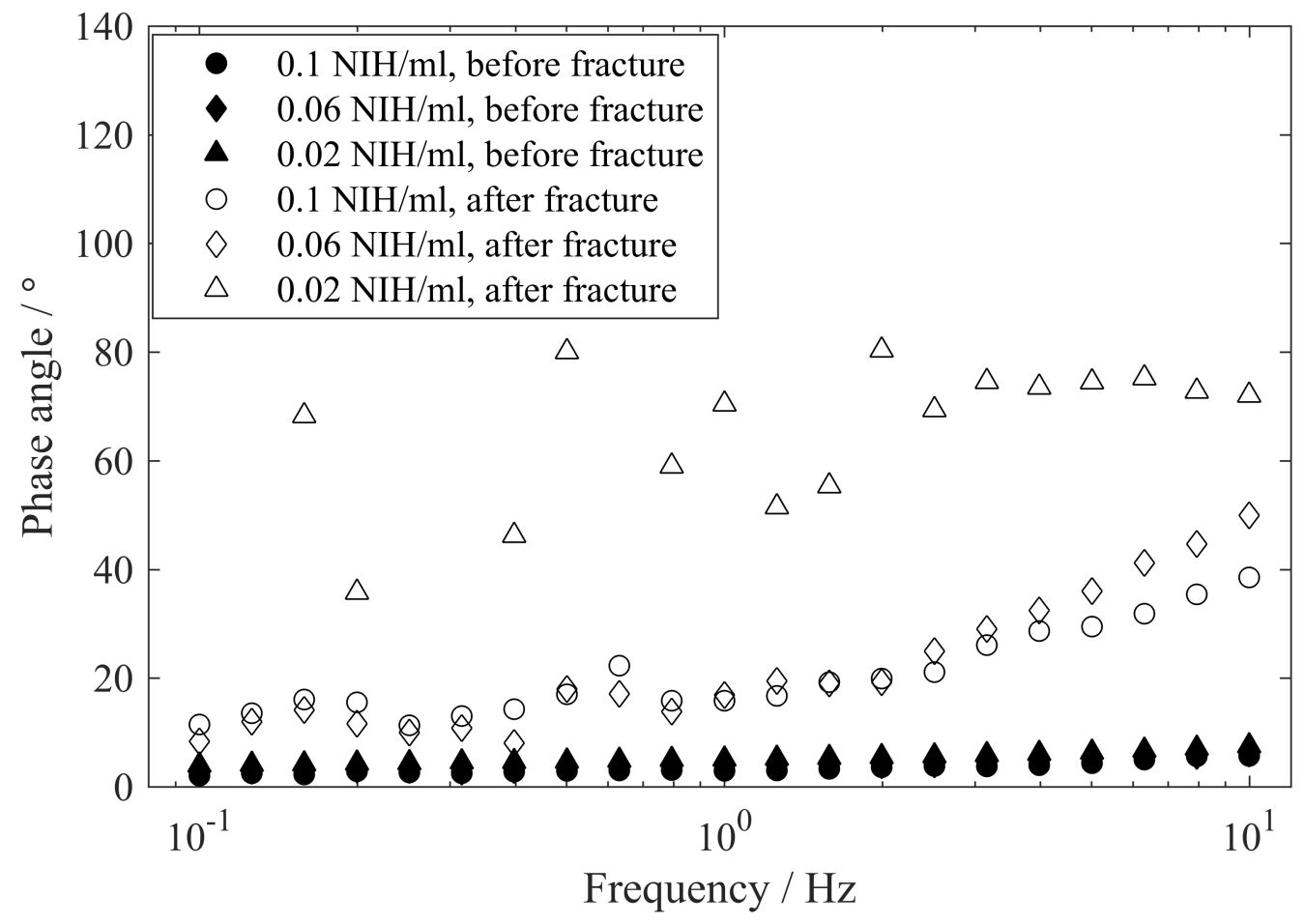

Figure 4.10 Frequency sweeps of the fully formed clot before the LAOS step, and after the fracture of the clot by LAOS

\subsubsection{Effect of strain history on the fracture properties of fibrin gels}

The linear and nonlinear region was identified in a strain sweep experiment (Figure 4.11a). The storage modulus was independent of the applied strain up to approximately $1.6 \%$, which corresponds to the critical strain. In Figure $4.11 \mathrm{~b}$ the results showed that the fracture stress (measured in Stage 3) is independent of the applied constant strain (applied in Stage 2) below $50 \%$ strain, above that the fracture stress is decreasing as the applied strain is increasing. 

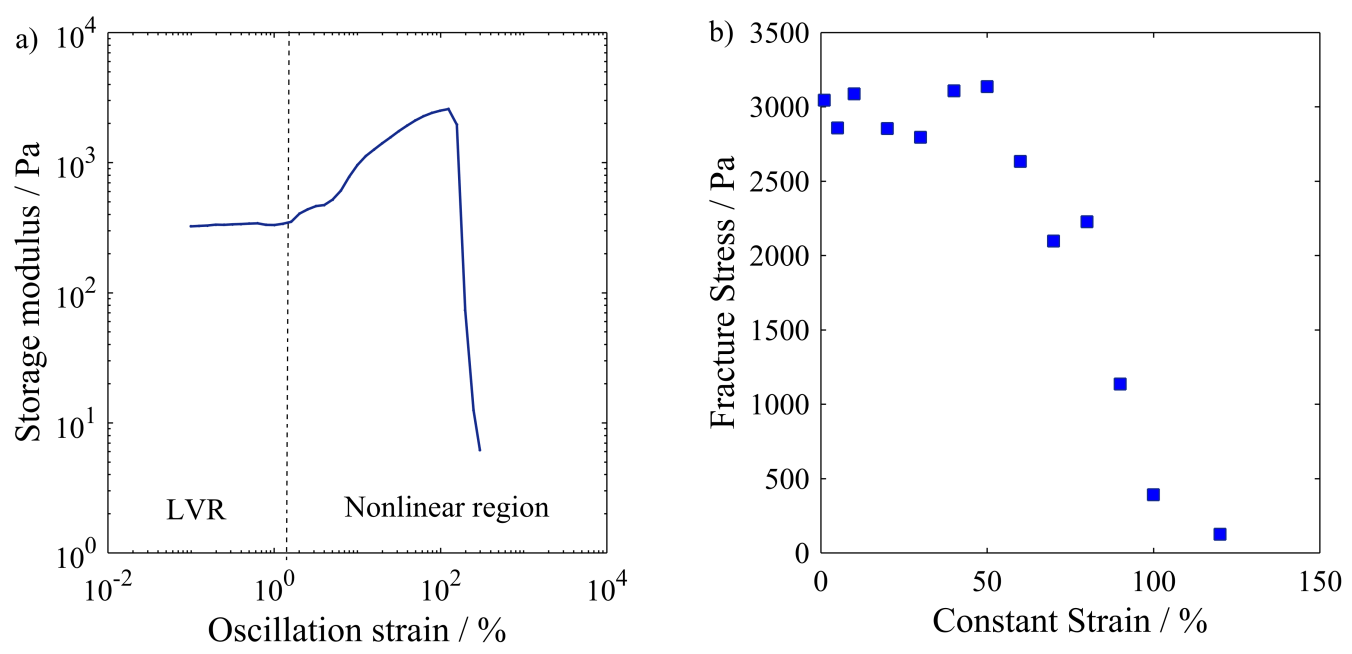

Figure 4.11 In a 'Strain Sweep' experiment the LVR (below $1.6 \%$ strain) and nonlinear region were identified (a). The fracture stress, which was measured in Stage 3 is plotted as a function of the applied constant strain amplitude in Stage 2 (b).

Figure 4.12 represents the stress response at different levels of applied strain over a period of time (i.e. time sweep) up until the material reached a steady state. In Figure 4.12a the applied strain of $1 \%$ is within the linear viscoelastic range of the material and the amplitude of the resulting stress is not changing. At a strain of $40 \%$ a nonlinear response is measured, as the value of critical strain that corresponds to the end of the linear viscoelastic region was $1.6 \%$, but the stress amplitude does not change over the time period studied (Figure 4.12b), similarly to the LVR response. At an applied strain of $60 \%$, the output stress amplitudes decreased during the first few cycles of oscillation, and then levels off at a stress of approximately $1400 \mathrm{~Pa}$ (Figure $4.12 \mathrm{c}$ ). As larger levels of strain are applied ( $80 \%$ or $100 \%$ applied strain), the initial measured stress levels are higher, and then slowly decrease over the time scale of the experiment (Figure 4.12d-e), up until the point of fracture, whereby a significant decrease in stress response is observed (Figure 4.12f). 

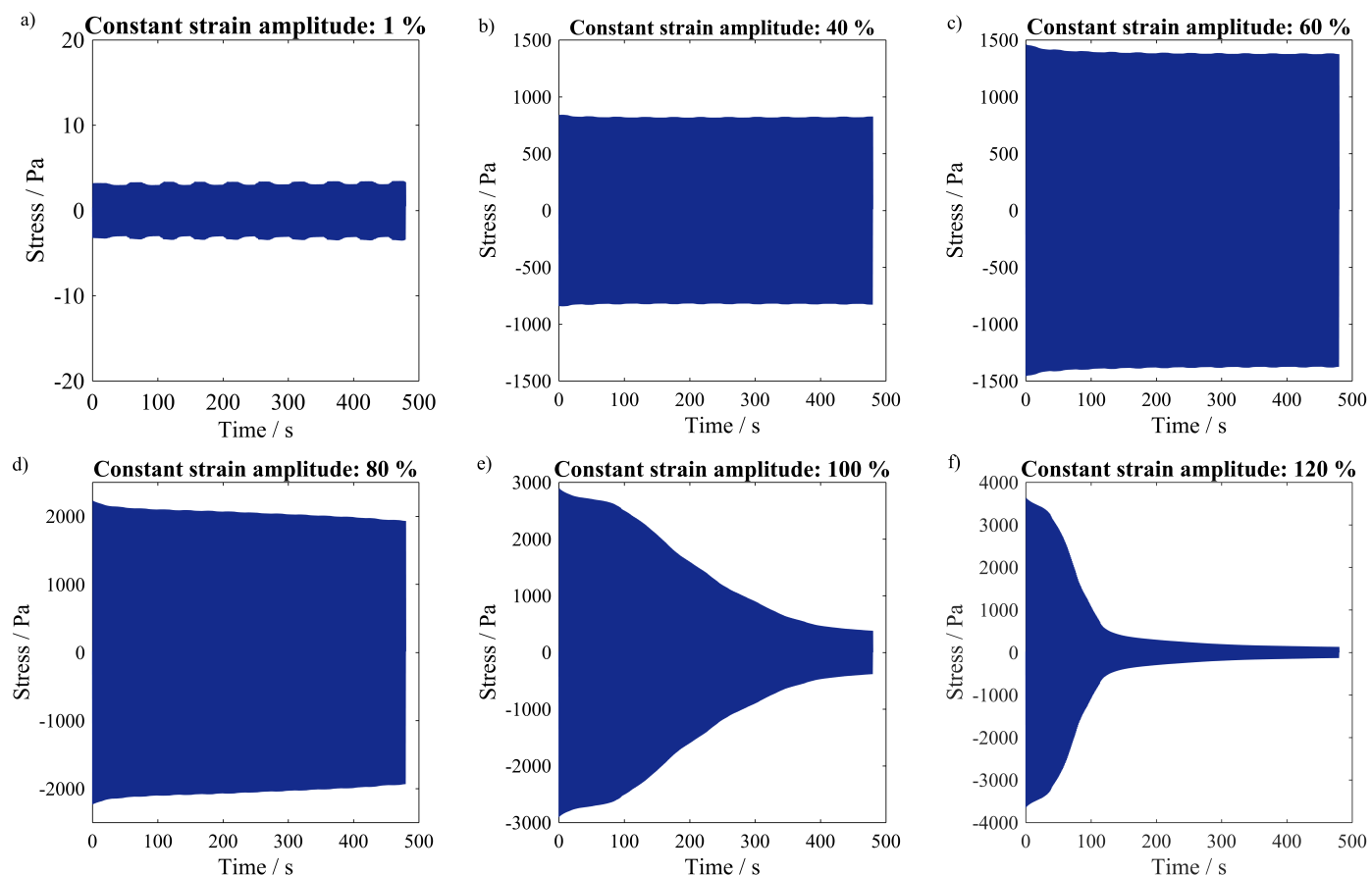

Figure 4.12 The measured stress responses for the applied constant strain amplitudes in Stage 2 as a function of time.

\subsubsection{Structural changes during LAOS}

Mature fibrin clots formed by the addition of various thrombin concentrations were subjected to increasing levels of strain amplitudes and allowed to reach a steady state. Following each incremental increase in applied strain, the linear viscoelastic properties of the clots were measured by SAOS at a strain level of $1 \%$. By the combination of alternating LAOStrain and SAOS measurements, reversible and irreversible changes in the mechanical properties of the material could be studied in response to the increasing levels of strain. Figure 4.13a illustrates the applied levels of strains, while Figure 4.13b-d represents the response of the materials in terms of oscillation stress, nonlinear moduli, $G_{L}^{\prime}$ and $G_{M}^{\prime}$, and storage modulus, $G^{\prime}$, respectively. Examination of nonlinear and linear moduli revealed different regions of behaviour within the nonlinear regime. In Region 1, the response of the material is nonlinear, as $G_{L}^{\prime}$ and $G_{M}^{\prime}$ are not equal, but following subsequent application of a strain level within the LVR the storage modulus returns to it's original value. In Region 2, the measured storage modulus, following the application of the LAOStrain at higher values of strain decreased slightly compared to the initial storage modulus. 
Whilst in Region 3, a decrease in the nonlinear moduli and oscillation stress in the LAOS measurement is seen and a dramatic decrease in storage modulus is found in the subsequent SAOS measurement.

a)

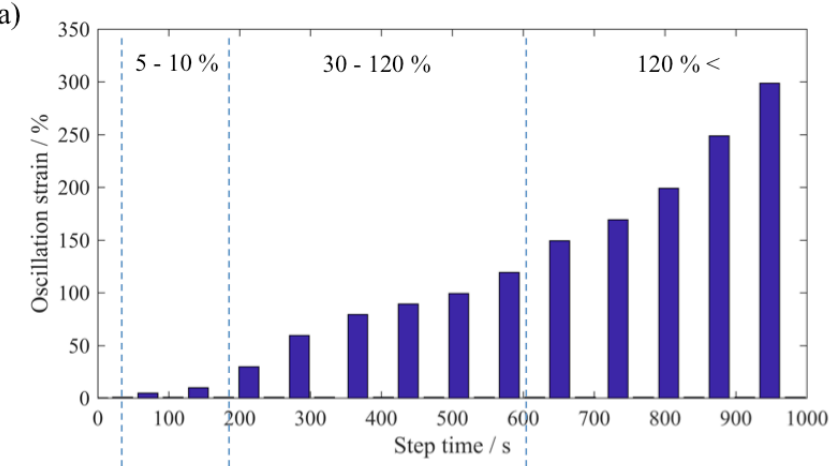

b)

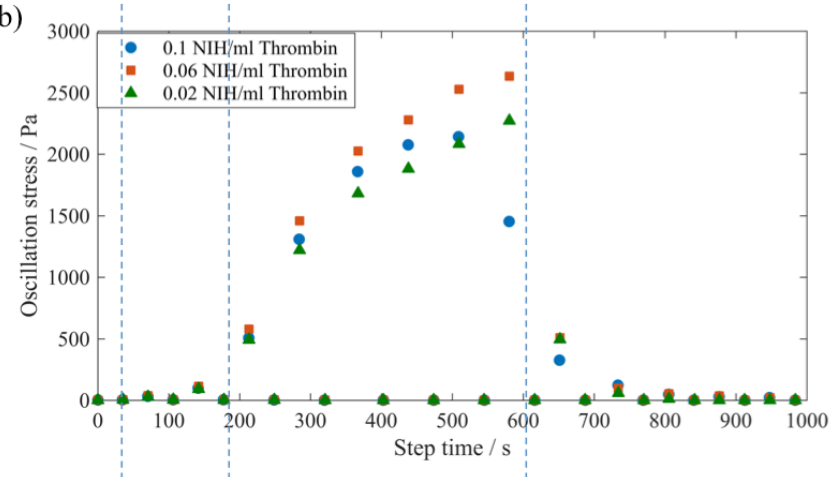

c)

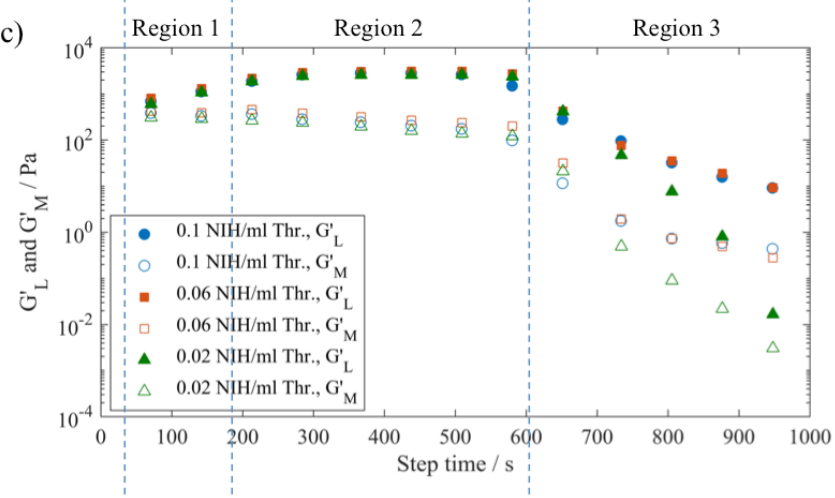

d)

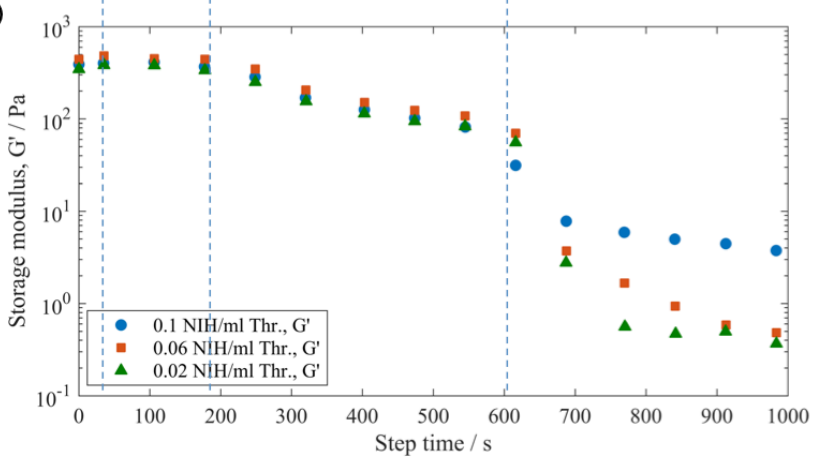

Figure 4.13 The (a) applied strain amplitudes and the response of the mature fibrin clot formed by the addition of different thrombin concentrations in terms of (b) stress, (c) $G_{L}^{\prime}$ and $G_{M}^{\prime}$ and (d) $G^{\prime}$ as a function of time. 


\subsection{Discussion}

Mature (i.e. fully formed) fibrin clots were characterised using LAOStrain. The fibrin network provides the underlying microstructural component of a blood clot and represents a model system of blood clotting (Shah \& Janmey 1997; Gersh et al. 2009; Undas \& Ariens 2011; Weisel \& Litvinov 2013b). The fibrinogen concentration used was fixed at $4 \mathrm{mg} / \mathrm{ml}$, which is the upper limit of the normal physiological range (Ogston \& Ogston 1966). Previous research by Curtis et al. showed that the thrombin concentration affects both the incipient clot microstructure (i.e. fractal dimension, $d_{f}$ ) and the structure of the fully formed clot; higher amounts of thrombin results in clots with a relatively higher fractal dimension and lower amounts of thrombin leads to clots formed with a relatively low fractal dimension (Curtis et al. 2013). The original hypothesis of this work stated that underlying microstructure of the clot influences propensity of the clot to fracture. Despite what was hypothesised, these results utilising a LAOStrain approach showed that the thrombin concentration did not affect the fracture properties, i.e. the fracture stress was independent of the thrombin concentration. We also explored whether fracture was more likely to occur at an early stage of clot formation. For this reason FXIII inhibitor was added to the fibrin gel to create a model system for an "immature" or "infant" clot, which does not have any crosslinks. The results suggest that these infant clots fractured at a lower stress value compared to the stress value measured for the mature clots and therefore the presence of crosslinks may resist the likelihood of embolism. However, similar to the results obtained on mature clots, the fracture stress of the infant clots did not change with changing thrombin concentration. In addition, the fracture stress did not show notable dependence on the maximum measured complex modulus, $G_{\text {max }}^{*}$, value in the SAOS step. Similarly, Munster et al. also found that there is a lack of correlation between the stiffness of the formed fibrin clot and the nonlinear properties, including rupture stress (Munster et al. 2013). Consequently, $G_{\max }^{*}$ is not a good indicator of fracture stress, therefore it cannot be considered as biomarker for embolism.

Frequency sweep measurements revealed vast differences between the rheology of unperturbed and fractured fully formed clots. This suggests that the proposed definition for fracture stress - maximum measured stress during the LAOStrain 
experiment - is an appropriate definition, and it is evident that the clot is indeed fractured at the fracture stress. The fracture stress measured in strain sweep experiments was found to be independent of the structure of the clot. The application of strain sweep measurements captured the eventual fracture of the fibrin clots, but it did not give a complete insight in to the process of fracture, as it did not reveal any information regarding the possible structural rearrangements of the fibrin clot, and how this might be affected by the structure. Therefore, the effect of strain loading history on the viscoelastic properties of mature fibrin clots was studied, and different regions were identified based on the stress response and eventual fracture of the sample.

Individual strain loading history measurements were conducted, in which the fully formed clot was subjected to a fixed level of strain up until the material reached a steady state (Stage 2), following which the fracture stress of the clot was measured in a strain sweep experiment (Stage 3). The fracture stress, which was measured in Stage 3, did not change up until the strain applied in Stage 2 exceeded $50 \%$. Above $50 \%$ strain (Stage 2), the strain history affected the fracture stress as determined in Stage 3. Increasing levels of strain imposed on the clot in Stage 2 resulted in lower fracture stress levels in Stage 3. At strain levels between $1.6 \%$ and $50 \%$ strain, the stress response was nonlinear, however the applied strain did not affect the fracture stress, which suggests that the nonlinearities might be attributed to the stretching of the fibrin fibres, and the deformation is reversible. While, above constant strain of 50 $\%$ in Stage 2, irreversible deformation occurs, this might be due to some localised fracture in the sample, which does not result in immediate fracture of the clot, but affects its structural stability, as the eventual fracture of these samples is affected in Stage 3. Examination of the stress response in Stage 2, reveals that the increasing levels of strain leads to an increase in stress response, and also increases the time period required to reach a steady state.

A protocol to investigate reversible and irreversible structural damage was used by applying alternating LAOStrain and SAOS experiments. These experiments captured the strain stiffening behaviour of the fibrin clot, as $G_{L}^{\prime}$ dominates over $G_{M}^{\prime}$ throughout the applied large strain levels. Previous studies that applied large strains to the fibrin clots also found evidence for the remarkable strain stiffening behaviour of the fibrin 
network and individual fibres (Liu et al. 2006; Kang et al. 2009; Munster et al. 2013; Münster et al. 2013; van Kempen et al. 2015; Keshavarz et al. 2017). Results found by combining and alternating LAOStrain and SAOS steps are in good agreement with the results found in the "individual" strain history measurements (See Chapter 4.4.3). Four distinct regions were identified, including the linear viscoelastic region (LVR) and three different regions within the nonlinear range. Nonlinear viscoelastic Region 1 was outside the LVR, however, the clots subjected to a level of strain within this region fractured at a similar fracture stress to those clots exposed to a strain within the LVR. Furthermore, the clots returned to their original linear viscoelastic behaviour following a step decrease in applied strain. This might be due to fully recoverable structural rearrangements, as fibrin fibres can be stretched threefold before breaking (Brown et al. 2009). In Region 2, the response is nonlinear and the material did not return to it's original linear viscoelastic behaviour following a step decrease in applied strain, possibly due to non-recoverable structural rearrangements (adaptation to stress) or localised fracture. Complete fracture of the clots occurred at the start of Region 3. Evidence for the presence of different characteristic regimes of fibrin were also reported by Munster et al. by investigating changes in the differential modulus, $K^{\prime}$, and by Keshavarz et al. by studying the stress response of the material (Munster et al. 2013; Keshavarz et al. 2017). Those studies applied strain ramps with a fixed shear rate for measuring the nonlinear behaviour and fracture of the fibrin clots. In contrast, the approach herein studied the effect of increasing levels of strains, by the application of alternating LAOStrain and SAOS, and the fibrin clot was allowed to reach a steady state in each step, and therefore the method herein is more physiologically relevant. Moreover, the rheological response measured in the SAOS steps provides information about any reversible or irreversible deformations of the fibrin network caused by the application of large strains in the LAOStrain steps.

These aforementioned regions are important because during thrombus growth the wall shear stress will increase and then decrease prior to the full vessel occlusion (Colace et al. 2012), and a knowledge of the regions could predict whether a clot will embolise or not. Depending on the level of stress imposed on the clot over time, the clot might adapt under shear stresses (as seen in Region 2), or fully fracture like the behaviour seen in Region 3. 


\subsection{Conclusion}

The effect of different thrombin concentrations was investigated, as it was hypothesised that different thrombin concentrations would create different mature clot microstructures, which would affect the levels of strain that bound each region. However, the onset of the different regions occurred at the same strain levels for all thrombin concentrations studied. This suggests that either the nonlinear properties of fibrin clots are independent of the applied thrombin concentration, or that the straincontrolled method of LAOS is not the most appropriate method for characterising fibrin clots formed with various thrombin levels. For this reason, the following chapter (Chapter 5) will investigate and demonstrate the LAOStress properties of fully formed fibrin clots. In addition, studying the behaviour of fibrin clots subjected to large stresses might be more physiologically relevant, compared to the behaviour of fibrin clots under large strains (Kroll et al. 1996; Colace et al. 2012). 


\section{LAOStress characterisation of fibrin gels}

The previous chapter entailed an investigation into the nonlinear viscoelastic behaviour of mature fibrin clots under imposed Large Amplitude Oscillatory Shear Strains. However, these controlled strain measurements failed to reveal differences in fibrin clots formed by the addition of various thrombin levels, and it is arguable that an investigation into the behaviour of fibrin clots under imposed stresses might be more physiologically relevant. Clots formed in vivo experience a shear stress due to haemodynamic forces or the flow of blood in vessels such as veins and arteries. The wall shear stress in veins can span several magnitudes $(0.1 \mathrm{~Pa}$ to $38 \mathrm{~Pa})$ depending on the size, branching and number of vessels and can be elevated in disease states (Zarins et al. 1983; Kroll et al. 1996). A study using microfluidic channels found that during the growth of a thrombus, the shear stress increases as the flow squeezes past the growing thrombus and then rapidly decreases (in a "pressure relief" mode, where blood is allowed to flow through unobstructed channels in parallel) at $80 \%$ of full occlusion (Colace et al. 2012). Therefore, it is important to consider the effect of both the vast range and progressive increase in stresses experienced by fully formed clots. Therefore, the work presented in this chapter explores the use of the LAOStress to characterise the nonlinear rheological properties of fully formed fibrin clots, including those formed with different incipient microstructures.

\subsection{Aims and objectives}

The purpose of this chapter was to develop the LAOStress technique to study the nonlinear viscoelastic properties of fully formed fibrin clots. This permitted an investigation into the effect of different incipient clot microstructures on the nonlinear properties, as measured by LAOStress. Fibrin clot structures were modified by the addition of different thrombin levels and by forming clots under a unidirectional shear stress using controlled stress parallel superposition (CSPS). 


\subsection{Materials}

Fibrin gels with final fibrinogen concentration of $4 \mathrm{mg} / \mathrm{ml}$ were used in the measurements as a model system for blood. Purified, plasminogen depleted human fibrinogen at least $95 \%$ clottable, and human thrombin were obtained from Enzyme Research Laboratories, UK. The fibrinogen was made up to stock solution of 44.54 $\mathrm{mg} / \mathrm{ml}$ by the addition of Tris-buffered saline, TBS (20 mM Tris, $\mathrm{pH} 7.4$ and $0.9 \%$ $\mathrm{NaCl}$, Sigma Aldrich, UK), and left to fully dissolve in a water bath at $37^{\circ} \mathrm{C}$. The thrombin was dissolved in distilled water and made up to a stock solution of 500 $\mathrm{NIH}$ Unit/ml. These stock solutions were decanted into small Eppendorf tubes and frozen at $-80{ }^{\circ} \mathrm{C}$ until required. Tween 20 (J. T. Baker, USA) was added to TBS to make up a 1 weight $\%$ stock solution of TBS-Tween 20 , which was stored at $4{ }^{\circ} \mathrm{C} .1$ M stock solution of $\mathrm{CaCl}_{2}$ (Sigma Aldrich, UK) was also stored at $4{ }^{\circ} \mathrm{C}$.

The appropriate volume of TBS-Tween 20, normal TBS, fibrinogen and $\mathrm{CaCl}_{2}$ were mixed together to make a final concentration of $4 \mathrm{mg} / \mathrm{ml}$ Fibrinogen, 0.1 weight $\%$ Tween 20 and $0.005 \mathrm{M} \mathrm{CaCl}_{2}$. The clotting was initiated by adding the required thrombin volume to produce a final thrombin concentration of $0.1 \mathrm{NIH} / \mathrm{ml}$. After appropriate mixing, the sample was transferred immediately onto the rheometer's lower plate.

\subsection{Methods}

\subsubsection{Structural changes during LAOStress}

Rheological measurements were conducted using an AR-G2 controlled stress rheometer (TA Instruments, UK) fitted with a cone and plate (40 $\mathrm{mm}$ cone, 0.0351 radian) measuring system. The lower geometry plate was maintained at a temperature of $24{ }^{\circ} \mathrm{C}$ for all the experiments conducted. In order to reduce effects of evaporation, the sample was sealed with a thin layer of low viscosity $(10 \mathrm{mPa} \mathrm{s})$ silicon oil. 
SAOS was applied to the sample for 40 times the gel time, allowing the clot to fully form, whilst the evolution of viscoelastic modulus was monitored during the clotting process (Curtis et al. 2013). Following the attainment of the fully formed clot, LAOStress measurements were performed on the same sample by applying continuous stress waveforms at a stress amplitude in the nonlinear viscoelastic range of the sample and a frequency of $1 \mathrm{~Hz}$ until the sample reached a steady state. Following the acquisition of LAOStress data, the sample was probed by small amplitude oscillatory stress measurements at a stress level of $5 \mathrm{~Pa}$ and a frequency of $1 \mathrm{~Hz}$ until it reached a steady state in order to provide a value of linear elastic compliance, $J^{\prime}$. Adherence of the SAOS measurements to the linear viscoelastic range of the sample was confirmed by the absence of a third harmonic response. The process of alternating LAOStress measurements at incrementally increasing stress amplitudes and SAOS measurements was performed until the sample macroscopically fractured leading the upper rheometer plate to over speed. A criterion was imposed throughout all experiments that steady state was reached once the difference in the strain response between consecutive waveforms was less that 2 $\%$ (for SAOS) or $0.2 \%$ (for LAOStress). This criterion ensured that point symmetry was observed in the Lissajous-Bowditch curves obtained from the LAOStress tests and the irreversible damage following relaxation of the sample was probed by SAOS (as shown in Chapter 4.4.1). The sample stress and strain response waveform datasets were obtained by the rheometer software (Trios) in a transient data collection mode at the software's default sampling rate $978 \mathrm{~Hz}$. Different clot microstructures as formed from different thrombin concentrations, ranging between 0.02 and $0.2 \mathrm{NIH} / \mathrm{ml}$, were also studied in a series of LAOStress experiments. Experiments were repeated three times at each thrombin concentration.

\subsubsection{Fibrin clots formed under Controlled Stress Parallel Superposition}

LAOStress properties of mature fibrin clots, formed under Controlled Stress Parallel Superposition (CSPS) in which the oscillatory and unidirectional components are parallel to each other (Badiei et al. 2015) were also tested. CSPS is a useful tool for measuring the rheological properties under an imposed shear flow, and can be considered as a simplification of the complex flow regime of blood within the blood 
vessels that also experiences the pulsatile flow dynamics of the circulatory system. Therefore clots formed in rheometer plates under conditions of both steady and pulsatile flow experience conditions that are more representative to those formed within the human body. Badiei et al. showed that the microstructure of the blood clot, described by fractal dimension, $d_{f}$, is sensitive to the unidirectional flow shear stresses. Compared to the $d_{f}$ value measured in SAOS (i.e. $d_{f}=1.75$ at zero unidirectional stress component, $\left.\sigma_{s}=0\right), d_{f}$ gradually increases with increasing unidirectional stress levels up until $d_{f}=2.20$ at $\sigma_{s}=0.141 \mathrm{~Pa}$ (Badiei et al. 2015). Therefore, CSPS enables formation of clots with different clot microstructures for the same level of thrombin.

Whilst there is no accumulative strain in SAOS, the accumulated unidirectional strain in CSPS gradually increases throughout the experiment. In the CSPS work herein the unidirectional shear rate varies in time under constant stress, as the rheological properties of the material change during gelation. Therefore, the resulting flow is described by its oscillatory $\left(\sigma_{0}\right)$ and unidirectional flow $\left(\sigma_{s}\right)$ components.

The shear stress in CSPS can be expressed as,

$$
\sigma(t)=\sigma_{s}+\left|G_{\|}^{*}\right| \gamma_{0} \sin \left(\omega t+\delta_{\|}\right)
$$

where $\sigma_{s}$ is the unidirectional flow component of stress, $\left|G_{\|}^{*}\right|$ is the parallel complex modulus, $\gamma_{0}$ is the oscillatory shear strain amplitude, $\omega$ is the angular frequency and $\delta_{\|}$is the parallel superposition phase angle. The parallel storage $\left(G_{\|}^{\prime}\right)$ and loss $\left(G_{\|}^{\prime \prime}\right)$ moduli can be calculated as,

$$
\begin{aligned}
& G_{\|}^{\prime}=G_{\|}^{*} \cos \left(\delta_{\|}\right) \\
& G_{\|}^{\prime \prime}=G_{\|}^{*} \sin \left(\delta_{\|}\right)
\end{aligned}
$$




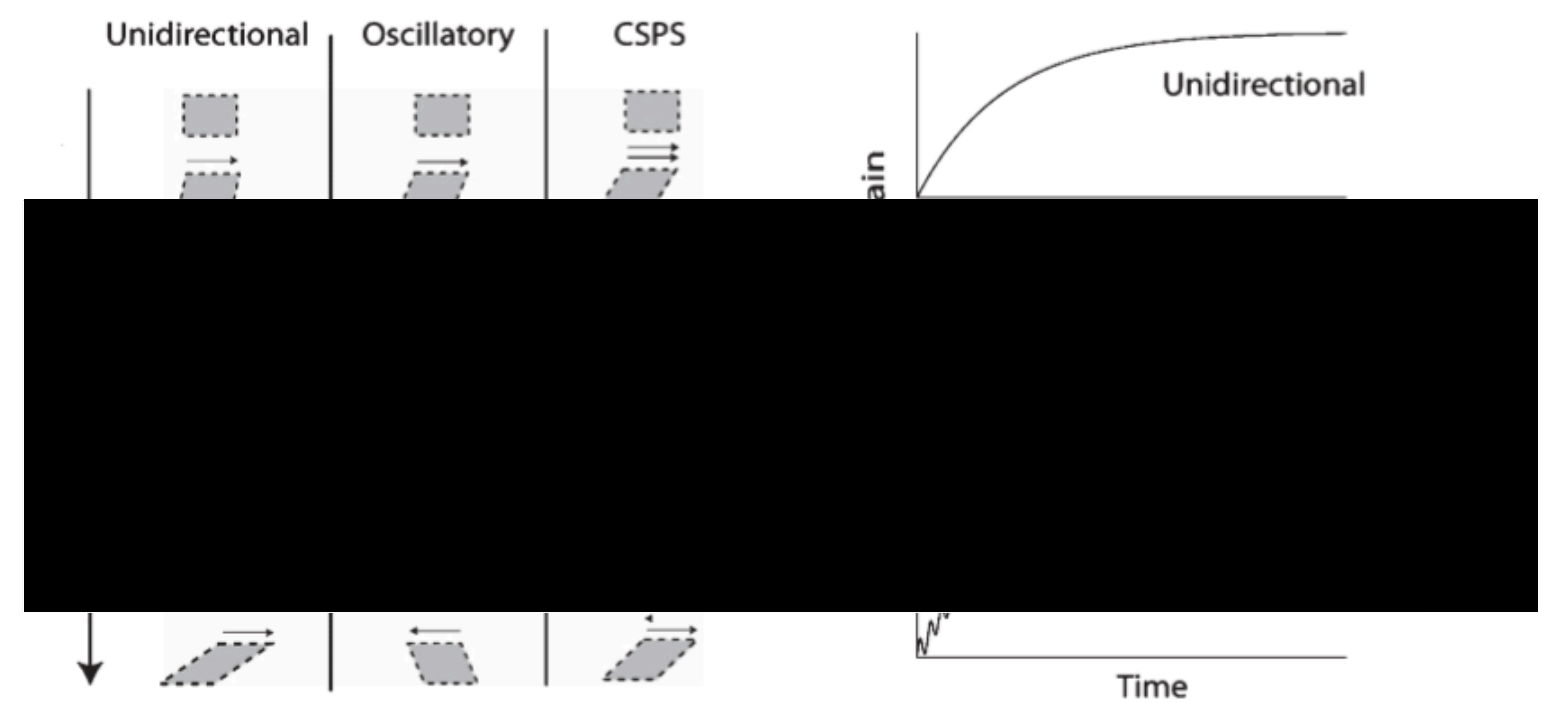

Figure 5.1 Graphical representation of CSPS (Badiei et al. 2015). The method combines unidirectional and oscillatory shear stresses, in which the directions of the resulting shear motions are parallel to each other. In oscillatory measurements the accumulated strain is zero, whilst in CSPS it is gradually increasing.

In the CSPS experiments, $\sigma_{s}=0.005 \mathrm{~Pa}$ or $\sigma_{s}=0.01 \mathrm{~Pa}$ was applied to the fibrin sample, and subjected to four different frequencies between 0.2 and $2 \mathrm{~Hz}$ in order to detect the Gel Point. Once the material underwent a transition through its Gel Point, the unidirectional flow shear rate, $\dot{\gamma}_{s}$, becomes negligible, which suggests that the value of $G_{\|}^{\prime}, G_{\|}^{\prime \prime}$ and $\delta_{\|}$are approximately the equal to their corresponding measures in SAOS (Badiei et al. 2015). The values of $d_{f}$ of the incipient clots formed under CSPS were calculated using the frequency independent value of $\delta_{\|}$. Experiments were repeated three times at each unidirectional stress level

\subsubsection{SEM imaging of fibrin clots}

SEM images of fully formed fibrin clots were taken using scanning electron microscope (Hitachi 4800 S, Hitachi High-Technologies Pte Ltd., Singapore). Fibrin clots were formed under SAOS between the rheometer plates, following that the clots were subjected to different levels of stresses according to the nonlinear regions observed in the structural changes study (Chapter 5.4.2). The sheared clots were carefully removed from the rheometer plates, and transferred to a Petri dish and washed three times with $50 \mathrm{mM}$ Sodium Cacodylate-HCl Buffer solution (pH 7.2 7.4, SPI Supplies) in every 10 minutes in order to remove excess salt. The samples were fixed overnight in $2 \%$ Glutaraldehyde (Sigma Aldrich, UK), following that the 
gels were dehydrated by adding a series of graded concentrations (30\% to $100 \%)$ of ethanol. The dehydrated samples were rinsed with $50 \%$ Hexamethyldisilazane solution (HMDS) (Sigma Aldrich, UK) in $100 \%$ ethanol for 10 minutes in a fume hood, and then rinsed three times with $100 \%$ HMDS and left to dry overnight. All samples were mounted, sputter-coated with a thin layer of gold-palladium (approximately $15 \mathrm{~nm}$ ) using an SEM coating unit E5100 (Polaron Instruments Inc., West Sussex, England) at $2.2 \mathrm{kV}$ and $20 \mathrm{~mA}$ for $1.5 \mathrm{~min}$, and examined in a Hitachi 4800 S scanning electron microscope (Hitachi 4800 S, Hitachi High-Technologies Pte Ltd., Singapore). Duplicates of each clot were made, and several fields on each clot were examined before choosing fields that were characteristics of the entire clot.

\subsection{Results}

\subsubsection{LAOStress raw data}

Figure 5.2 shows an example of sample stress waveforms (upper) and strain response waveforms (lower) at an applied stress level of (a) $100 \mathrm{~Pa}$ (slightly into the nonlinear viscoelastic range of the sample) and two higher levels of applied stress (b) $500 \mathrm{~Pa}$ and (c) $1500 \mathrm{~Pa}$. 

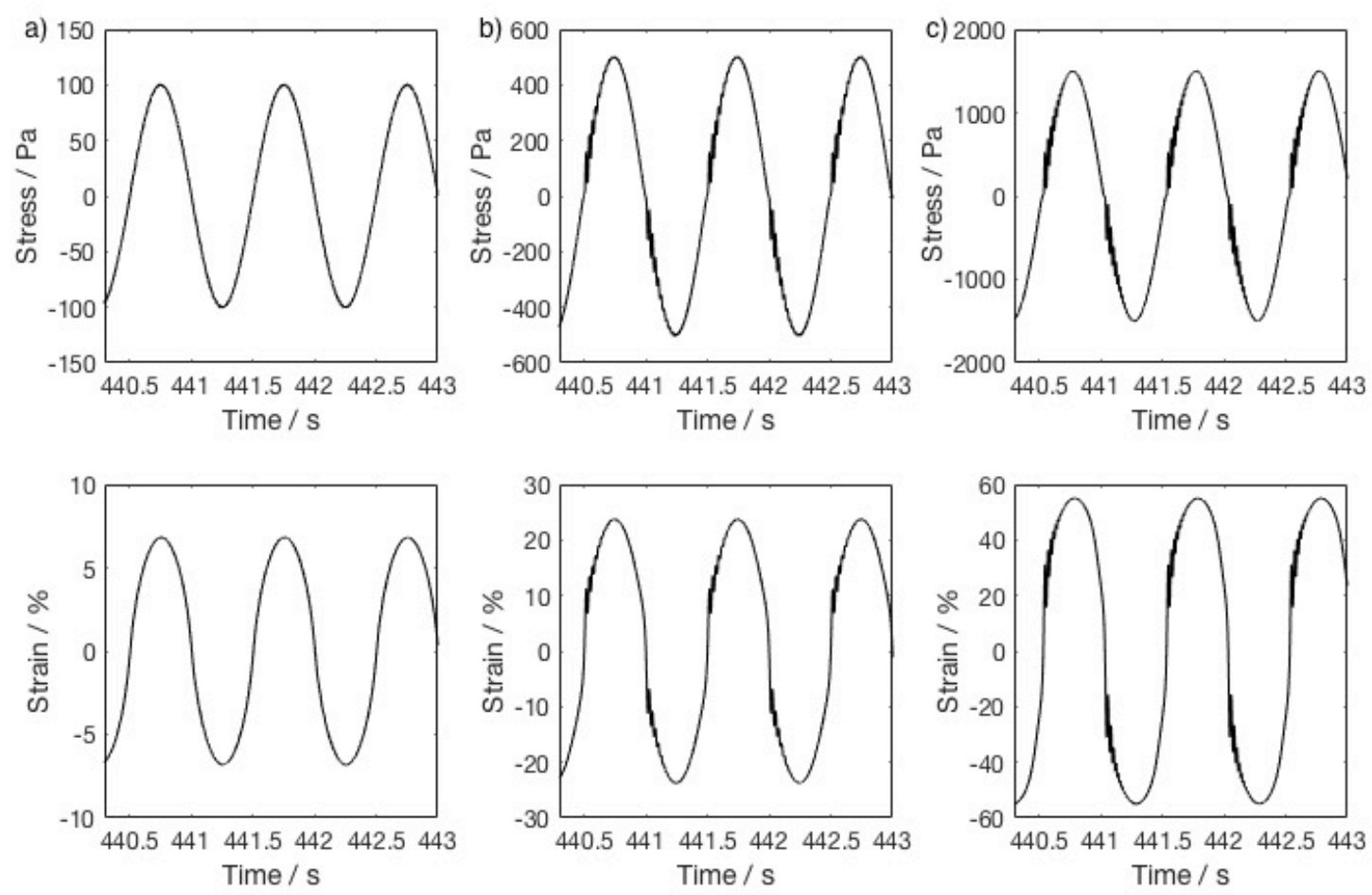

Figure 5.2 Sample stress (upper) and strain (lower) response waveforms obtained from the rheometer software (Trios) at input stress levels of (a) $100 \mathrm{~Pa}$, (b) $500 \mathrm{~Pa}$, and (c) $1500 \mathrm{~Pa}$. These waveforms consist of discrete data points at a sampling rate of $978 \mathrm{~Hz}$.

The dimensionless inertia number, In, proposed by Dimitriou et al. was calculated for all LAOStress measurements and $I n \ll 0.05$ throughout, indicating that instrument inertia alone did not significantly affect the total torque signal. However, at increasing levels of applied stress, the sample stress and strain response waveforms contained artifacts due to a source of ringing, and the waveforms were more affected by the ringing as the applied stress increases. This ringing is possibly attributable to a resonance caused by a coupling of inertial and elastic forces at relatively large levels of applied stress (Baravian et al. 2007).

Ringing was observed in both sample stress and strain waveforms in the LAOStress measurements. Previous studies have reported inertio-elastic ringing in creep experiments but this is the first evidence of ringing encountered in an oscillatory experiment (Yao et al. 2008; Ewoldt et al. 2011; Ewoldt \& McKinley 2007). Therefore, the purpose of the following set of experiments was to investigate the source of ringing by comparing the ringing behaviour in both creep and LAOStress experiments of the same material. 


\subsubsection{Inertio elastic ringing}

The controlled stress rheometer's instrument inertia creates experimental artefacts under transient conditions when the torque is measured at a moving boundary with unsteady motion. Acceleration is an unsteady instrument motion as it requires a certain amount of torque, therefore the measured torque is influenced by both the material deformation and instrument acceleration. Therefore, these artefacts can be present in oscillatory tests performed at higher frequencies, or in the short time data of step tests (Ewoldt et al. 2015).

This problem might be corrected if the instrument inertia is known, and in some cases the inertia correction could be negligible. However, in the case of creep experiments, the instrument inertia can also cause free oscillations and significantly alter the displacement response. This includes the time required to accelerate (which is why it affects the short time data), and free oscillations as the sample elasticity couples with the finite instrument inertia to "ring" at a resonant frequency, similarly to a mass at the end of a spring, and this phenomenon is called inertio-elastic ringing. Careful analysis of the inertio-elastic oscillations can be used to characterise the materials linear and nonlinear viscoelastic properties from the values of measured frequency (elasticity) and periodic decay (dissipation, viscous properties) of the free oscillations (Ewoldt et al. 2015).

In order to avoid invalid data in oscillatory shear due to instrument inertia, the material torque has to be larger than the instrument-inertia torque (Ewoldt et al. 2015).

$$
\begin{gathered}
T_{\text {material }}>T_{\text {inertia }} \\
\frac{G \gamma_{0}}{F_{\tau}}>I \theta_{0} \omega^{2} \\
G>\frac{I F_{\tau}}{F_{\gamma}} \omega^{2}
\end{gathered}
$$


In the case of a cone and plate geometry

$$
G>\frac{I 2 \pi R^{3}}{3 \beta} \omega^{2}
$$

Where the variable $G$ represents either $G^{\prime}$ or $G^{\prime \prime}$ in oscillation, $I$ is the instrument inertia created by any moving part of the instrument, $R$ is the cone radius, $\omega$ is the frequency, and $\beta$ is the cone angle. As long as this criterion holds, the measured data is acceptable, beyond this point (i.e. high frequency data) inertia corrections are required and the instrument inertia has to be subtracted from the sample inertia. This criterion was met in all LAOStress experiments conducted.

Inertio-elastic ringing behaviour was observed in the LAOStress experiments; therefore this behaviour was further examined. In order to compare inertio-elastic ringing in creep to the ringing observed during large stress oscillations; LAOStress experiments were performed on the mature fibrin clot by the application of a sinusoidal stress waveform with constant large stress amplitude (from $500 \mathrm{~Pa}$ to 1000 $\mathrm{Pa}$ ), until steady state was reached. Following that, a creep experiment was conducted on the same clot with different stress levels (from $500 \mathrm{~Pa}$ to $1000 \mathrm{~Pa}$ ), equivalent to the applied large stress in the LAOStress step.

\subsubsection{Comparison of inertio-elastic ringing in creep and in LAOStress}

Figure 5.3 represents the observed inertio-elastic ringing behaviour detected in both creep (Figure 5.3a) and in LAOStress (Figure 5.3b) measurements as expressed in the values of compliance. These values were then used to analyse the inertio-elastic ringing. 

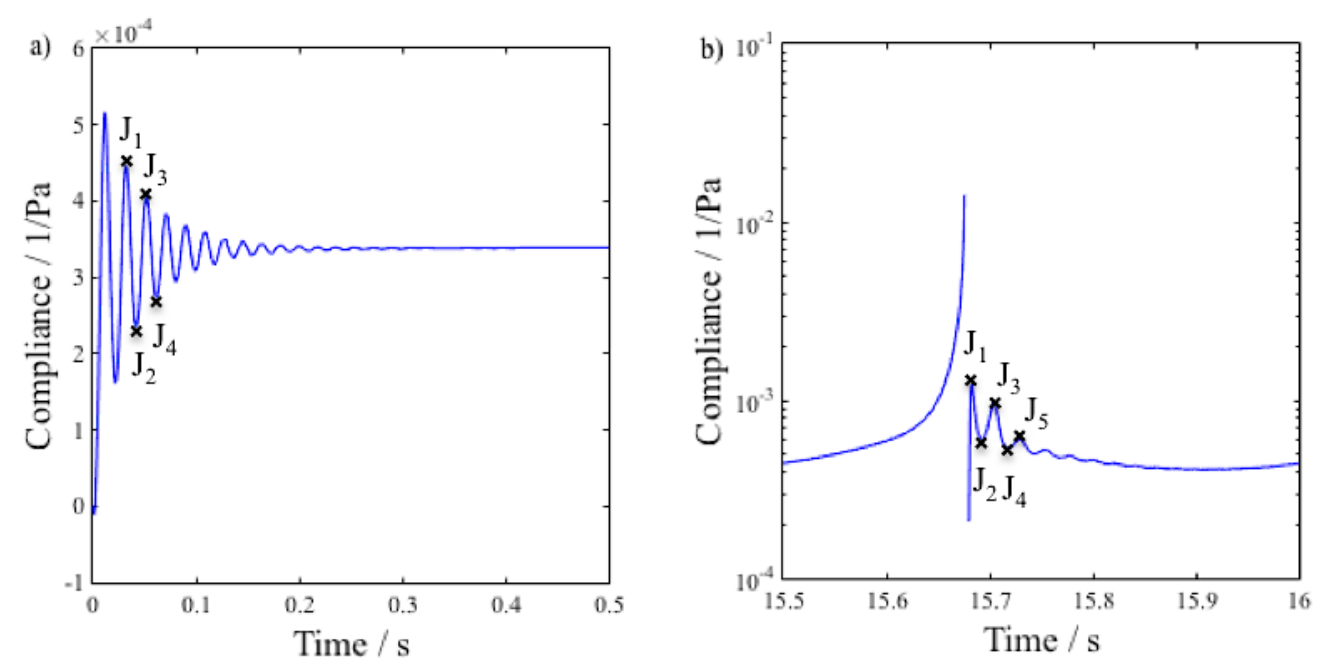

Figure 5.3 Inertio-elastic ringing observed in a mature fibrin clot (a) in creep and (b) in LAOStress.

By analysing the ringing waveform in creep, inertio-elastic ringing parameters, such as inertio-elastic ringing frequency and logarithmic decrement can be calculated:

$$
\begin{gathered}
\omega_{*}=\frac{2 \pi}{t_{4}-t_{1}} 1.5 \\
\Delta=2 \ln \left(\frac{J_{1}-2 J_{2}+J_{3}}{-J_{2}+2 J_{3}-J_{4}}\right)
\end{gathered}
$$

This approach was also applied to LAOStress data. In the case of creep experiments, the exponential decay envelope (i.e. two theoretical curves, one connecting the peaks and the other connecting the local minimums in the ringing) is symmetrical; however in oscillations the applied stress increases at the point at which the ringing is observed, and causing the exponential decay envelope to be non-symmetrical. In order to account for this behaviour an averaged value was used for calculating the logarithmic decrement in LAOStress, such as,

$$
\begin{aligned}
& \Delta_{1}=2 \ln \left(\frac{J_{1}-2 J_{2}+J_{3}}{-J_{2}+2 J_{3}-J_{4}}\right) \\
& \Delta_{2}=2 \ln \left(\frac{J_{2}-2 J_{3}+J_{4}}{-J_{3}+2 J_{4}-J_{5}}\right)
\end{aligned}
$$




$$
\Delta_{\text {LAOStress }}=\frac{\Delta_{1}+\Delta_{2}}{2}
$$

The calculated inertio-elastic ringing frequency and logarithmic decrement values were similar in both creep and LAOStress measurements (Figure 5.4). The inertioelastic ringing frequency is increasing with increasing applied stress levels in the case of both creep and LAOStress. The logarithmic decrement is also dependent on the applied stress, as higher level of applied stress leads to increased logarithmic decrement.
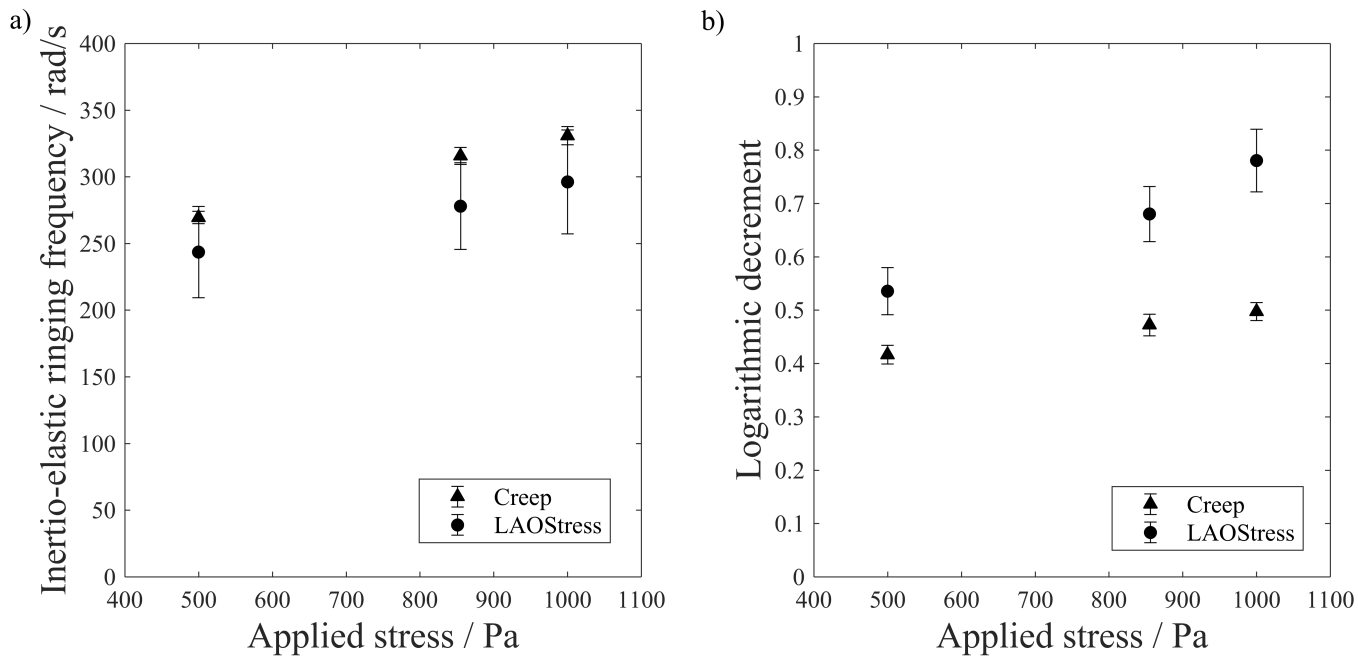

Figure 5.4 Results of the inertio-elastic ringing analysis: (a) inertio-elastic ringing frequency, and (b) the logarithmic decrement are plotted as a function of applied stress in creep and in LAOStress.

Differential (tangent) moduli were calculated using the ringing parameters:

$$
\begin{gathered}
K^{\prime} \approx \frac{I \omega_{*}^{2}}{c}\left(1+\left(\frac{\Delta}{2 \pi}\right)^{2}\right) \\
K^{\prime \prime} \approx \frac{I \omega_{*}^{2}}{c}\left(\frac{\Delta}{\pi}\right)
\end{gathered}
$$

Where $c$ is the geometry factor, calculated as $c=F_{\gamma} / F_{\sigma}$.

The differential moduli, $K^{\prime}$ and $K^{\prime \prime}$, increase with increasing levels of applied constant stress, which validates the strain stiffening behaviour of the fibrin gels in the 
nonlinear region (Figure 5.5). The inertio-elastic ringing analysis of both creep and LAOStress data resulted in commensurate $K^{\prime}$ and $K^{\prime \prime}$ values suggesting that the ringing in the waveforms is due to a material property. The similarity of the values of differential moduli also indicates that the same artefact is present in the creep and LAOStress measurement and this artefact being a consequence of inertio-elastic coupling.

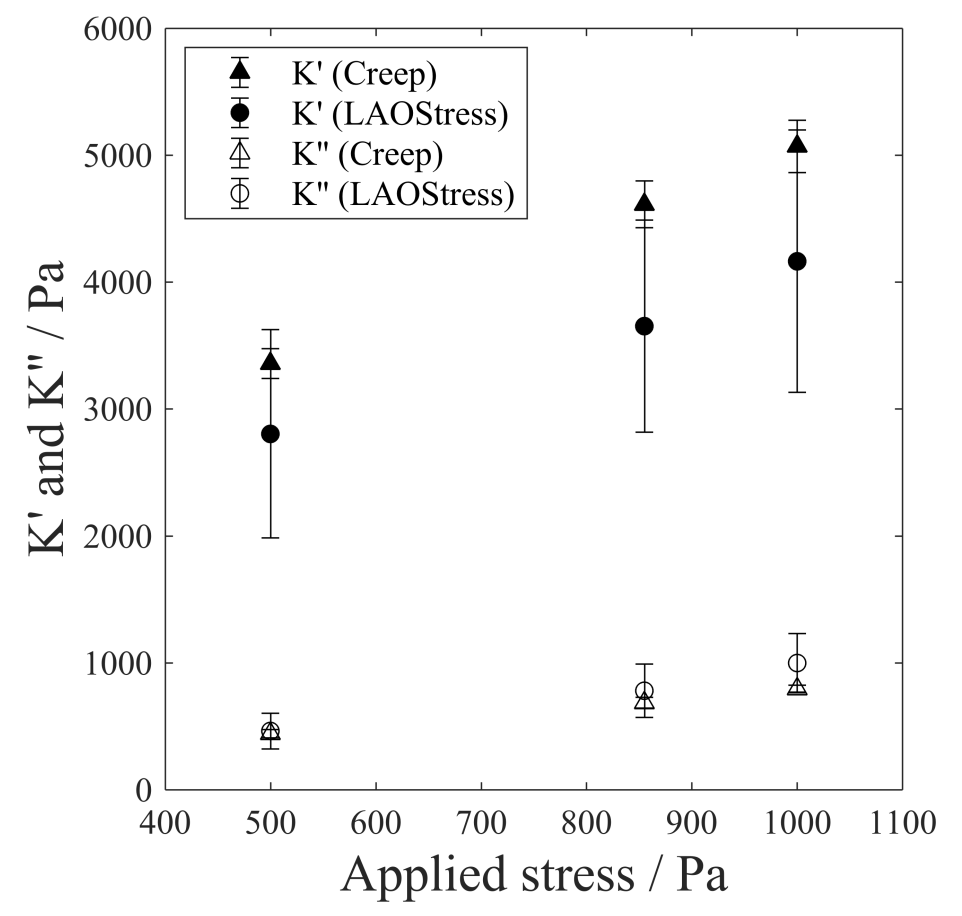

Figure 5.5 $\boldsymbol{K}^{\prime}$ and $\boldsymbol{K}^{\prime \prime}$ calculated using the inertio-elastic ringing analysis in creep and in LAOStress as a function of applied stress levels.

\subsubsection{LAOStress data processing}

In Lissajous-Bowditch plots of the raw stress and raw strain datasets, it is evident ringing leads to substantial artefacts including the presence of secondary loops (Figure 5.6). Observation of the Lissajous-Bowditch plot indicated that the ringing particularly affects the value of zero stress elastic compliance, $J_{M}^{\prime}$, calculated from the tangent of the curve at zero stress, which is likely to be largely elevated as a consequence. Therefore, these Lissajous plots constructed from raw data cannot be used for calculating meaningful and accurate values of nonlinear compliances. Therefore the ringing must be eliminated from both stress and strain waveforms 
before analysis of the Lissajous-Bowditch plots.

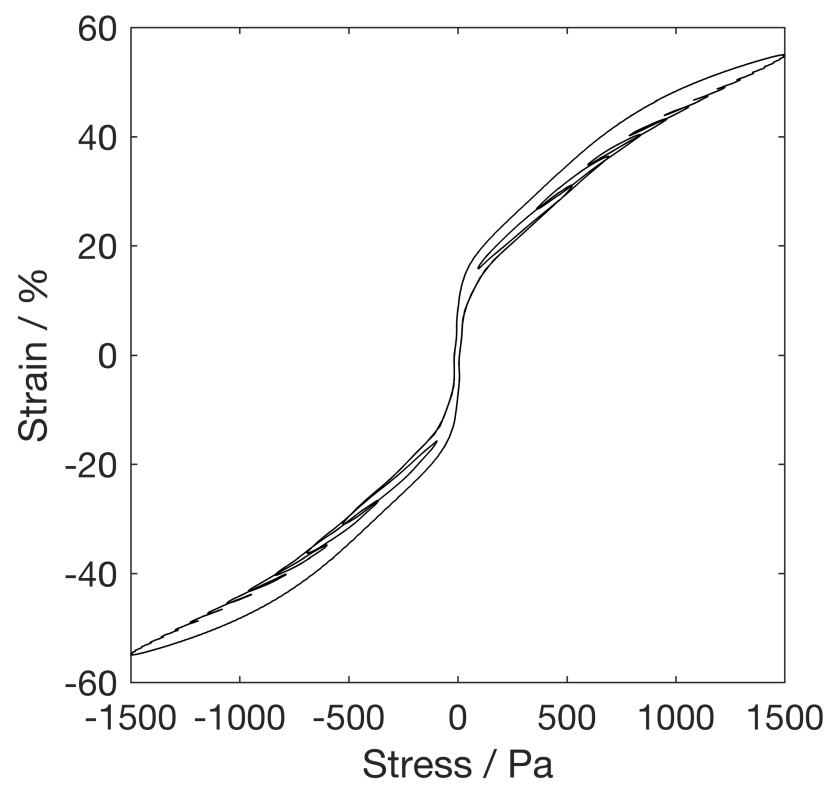

Figure 5.6 Example of a Lissajous-Bowditch plot constructed using the raw stress and strain waveforms for input stress level of $1500 \mathrm{~Pa}$.

In an attempt to eliminate the effects of ringing, the sample stress and strain response waveform datasets (obtained from Trios) were smoothed to a cubic spline function (specifically a piecewise cubic polynomial computed from a smoothing parameter $p$ $=0.99999252$ ) using the Curve Fitting Toolbox in MATLAB. The datasets were then reconstructed from the smoothing function by sampling at a rate identical to the original dataset $(978 \mathrm{~Hz})$. Figure 5.7 displays an example of the resulting smoothed waveforms. 

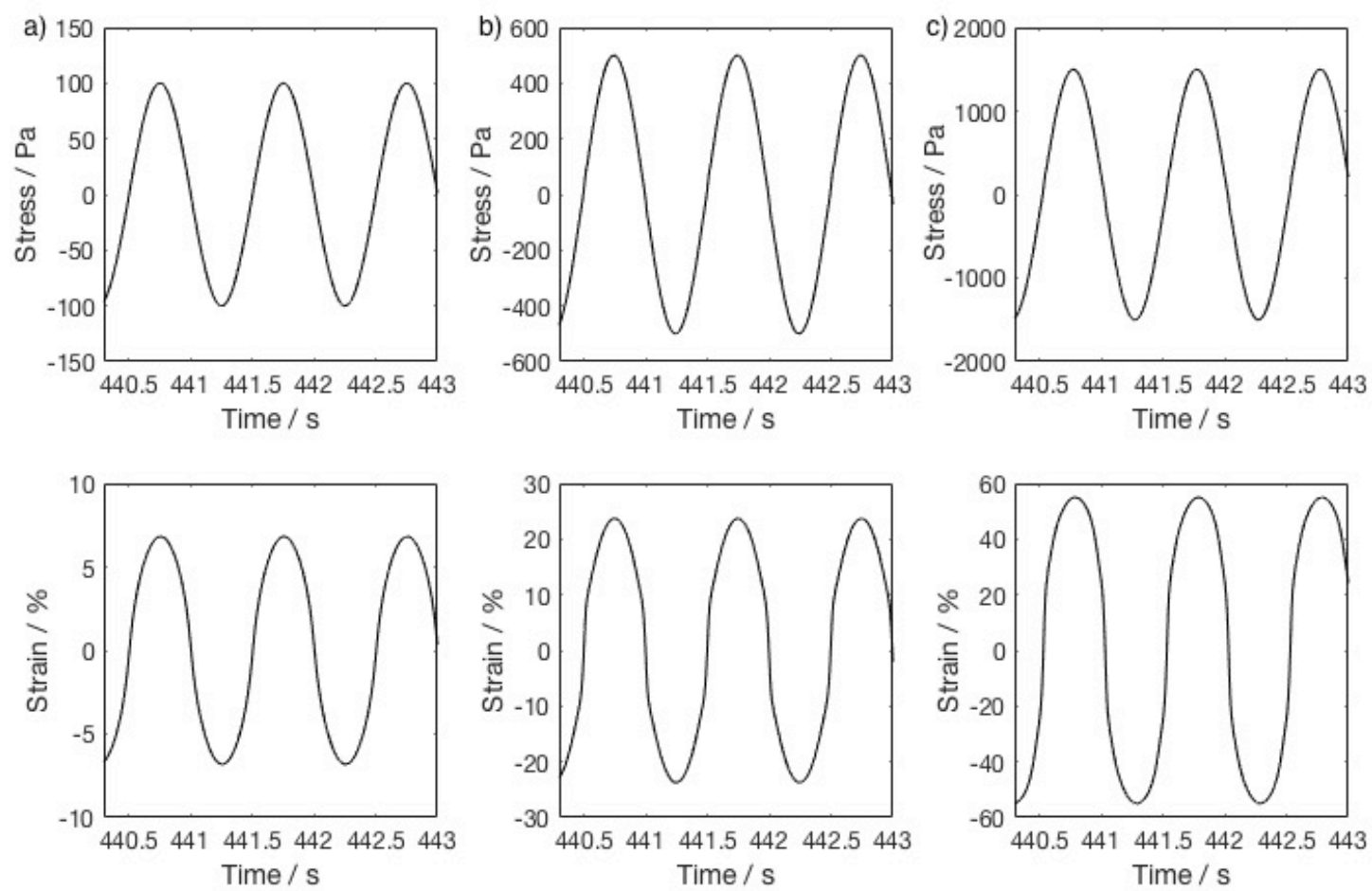

Figure 5.7 Smoothed sample stress (upper) and strain (lower) response waveforms obtained from the rheometer software (Trios) at input stress levels of (a) $100 \mathrm{~Pa}$, (b) $500 \mathrm{~Pa}$, and (c) $1500 \mathrm{~Pa}$. These waveforms consist of discrete data points at a sampling rate of $978 \mathrm{~Hz}$.

\subsubsection{Fourier transform analysis}

The process of smoothing eliminates any noticeable effects of ringing. However, in order to ensure that nonlinearities due to the sample response (which manifest as odd harmonics) are not affected by the process of smoothing, the datasets were subjected to Fourier analysis and the resulting frequency spectra were compared. To this end, the strain and stress datasets were trimmed to contain 15,000 data points which contain more than 15 complete cycles (Figure 5.8). 


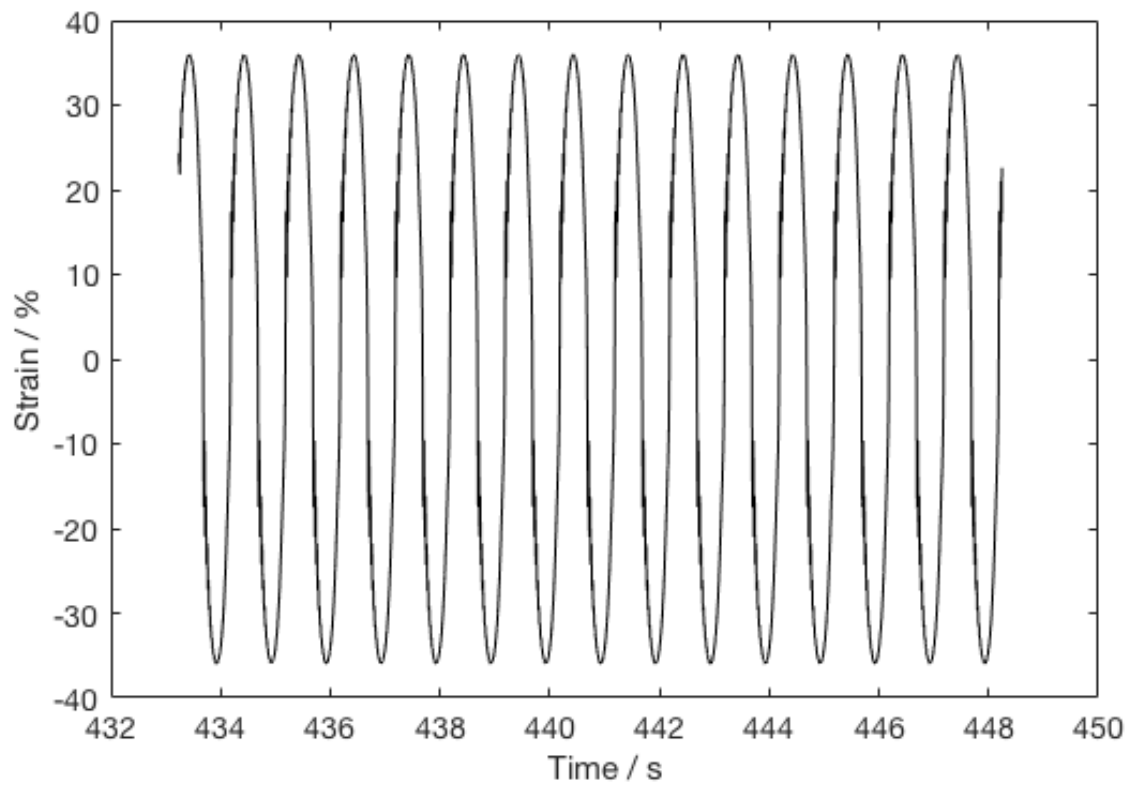

Figure 5.8 An example of the trimmed strain waveform that contains 15,000 data points, which is equivalent to 15 complete cycles.

The datasets were windowed using a Hanning window function (Figure 5.9). This is a useful tool for analyzing large data sets and signals that do not start and end in full cycles.

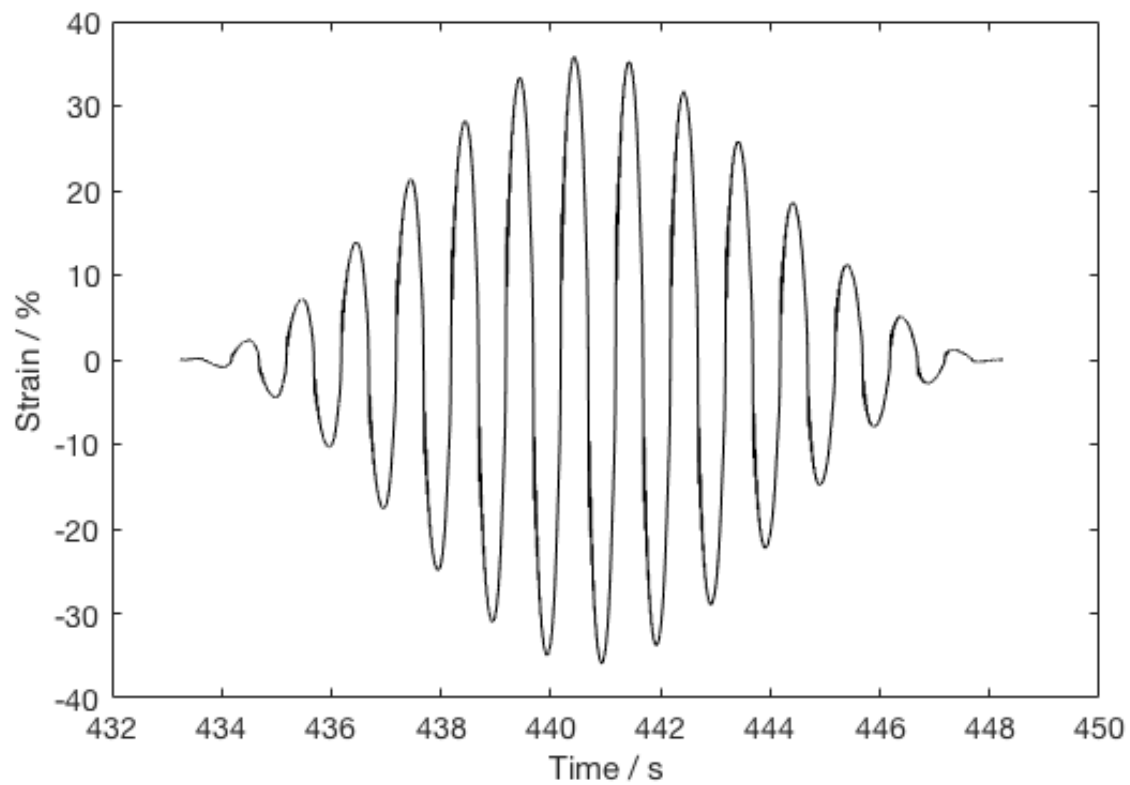

Figure 5.9 Example of a strain waveform that is windowed using Hanning window function. 
The datasets were also zero padded with 5,000 data points in order to adjust the resolution of the frequency spectrum to $0.05 \mathrm{~Hz}$ (Figure 5.10), which is the ratio of the sampling frequency, $F_{s}$, and the number of data points in the Fourier spectra.

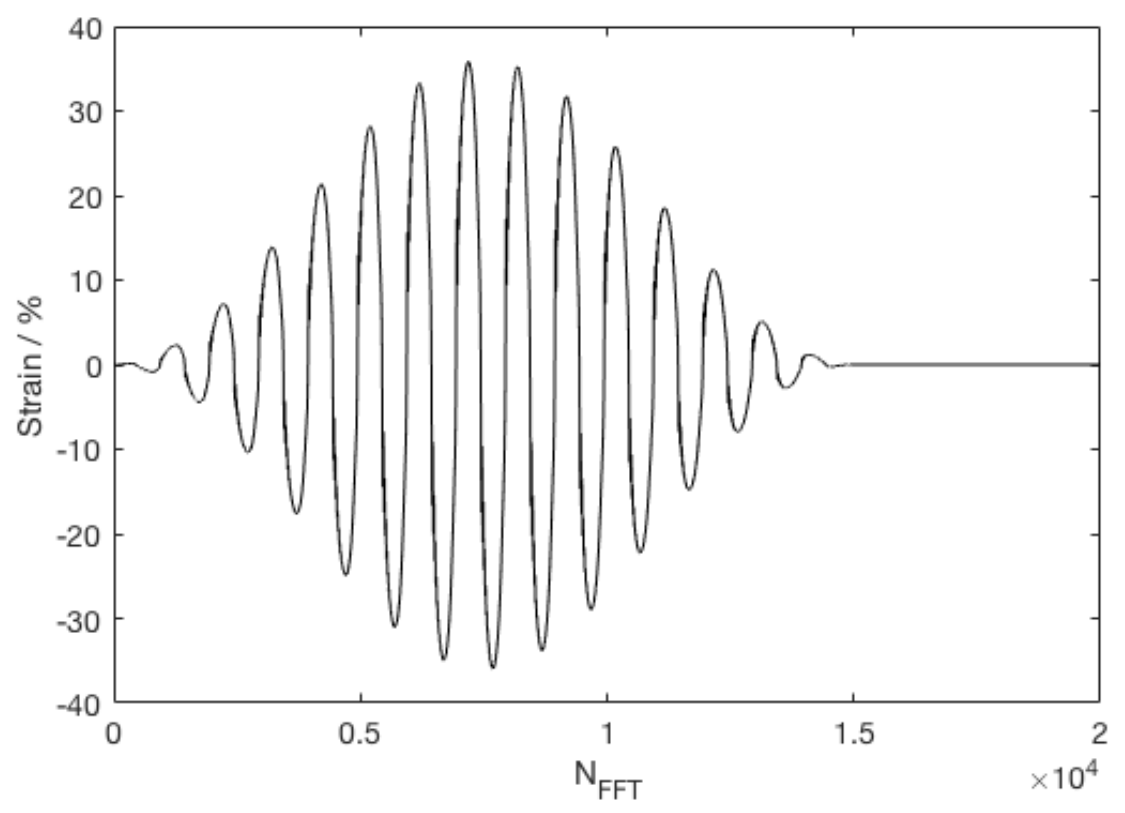

Figure 5.10 Example of a strain waveform that is windowed using Hanning window function and zero padded with 5000 data points.

The windowed and zero padded datasets were analysed in MATLAB using a discrete Fourier transform. The number of Fourier spectra points $\left(N_{F F T}\right)$ was converted into frequencies in $\mathrm{Hz}$ unit, and the magnitudes of the spectra were normalised to the first harmonic, hence the Fourier spectra shows peaks at odd integer numbers.

Figure 5.11 shows the frequency spectra of the sample stress and strain response waveforms for the original (i.e., unprocessed) data and smoothed data at three different levels of applied stress (a) $100 \mathrm{~Pa}$, (b) $500 \mathrm{~Pa}$, and (c) $1500 \mathrm{~Pa}$. 

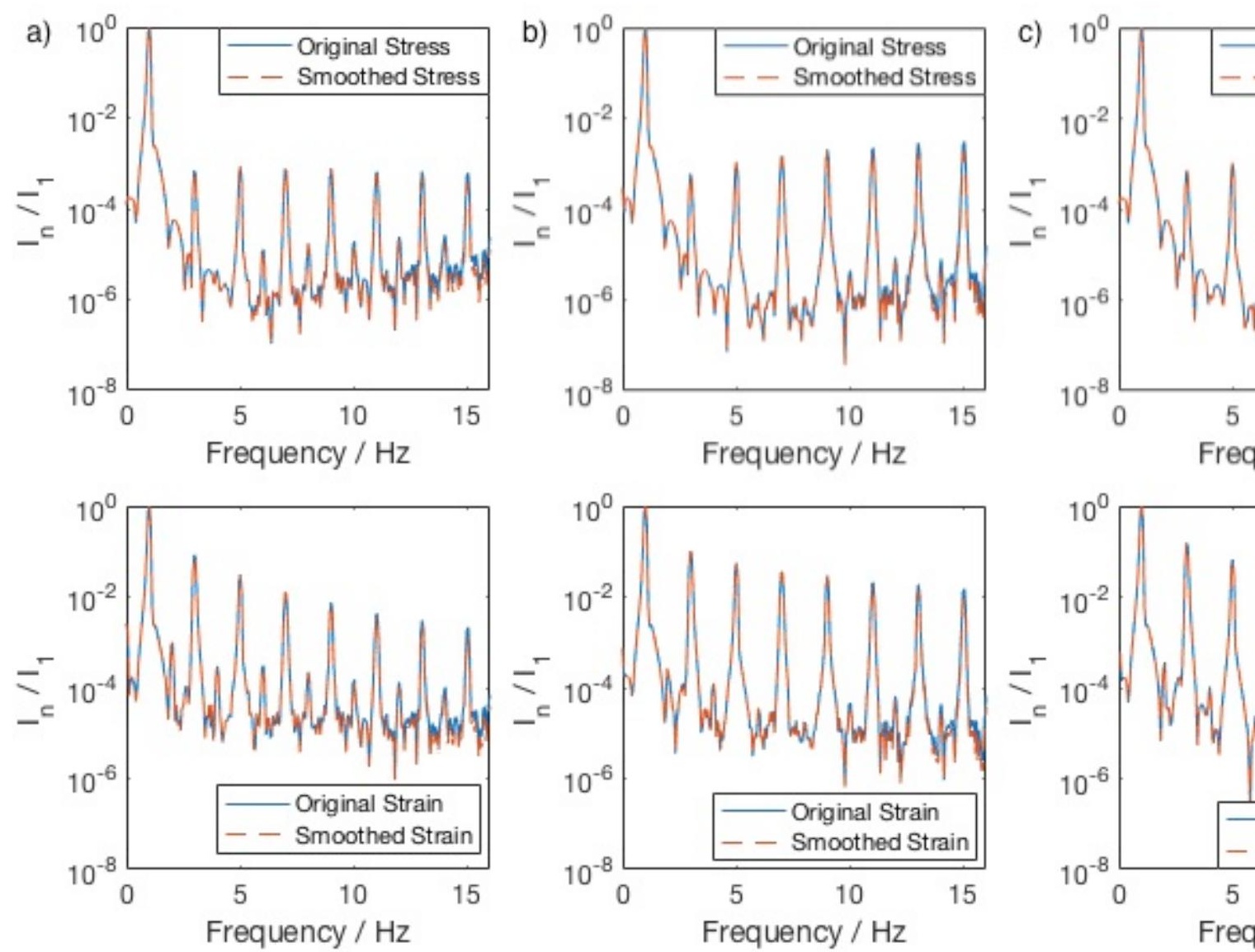

Figure 5.11 Comparison of frequency spectra obtained from discrete Fourier transforms of the original (blue solid line) and smoothed (red dottec (upper) and strain response (lower) waveforms at input stress levels of (a) $100 \mathrm{~Pa}$, (b) $500 \mathrm{~Pa}$, and (c) $1500 \mathrm{~Pa}$. The magnitudes of the spectra ( $\mathrm{I}_{\mathrm{n}}$ ) fundamental harmonic response $\left(\mathrm{I}_{1}\right)$. The fundamental frequency is $1 \mathrm{~Hz}$. 
The frequency spectra of the strain response are dominated by the fundamental (imposed) frequency and contain odd harmonics, confirming that the measurement is within the nonlinear viscoelastic regime (Giacomin \& Dealy 1993). The magnitude of the even harmonics was negligible confirming that the sample was non-transient and had reached a steady state (Atalik \& Keunings 2004). It is noticeable that some harmonic distortion in the sample stress waveform is apparent, including at the lowest stress shown where there was no ringing encountered. These nonlinearities will be transmitted to, and replicated in, the corresponding strain response. However, the magnitude of the odd harmonics in the strain response is much greater than the sample stress indicating that the rich nonlinearity of the sample is being captured. In order to estimate the degree of system (i.e. the sample's) nonlinearity, total harmonic distortion (THD) was calculated for the original and smoothed signals. THD can be used for setting a criterion for linearity, and it is expressed as the ratio of the higher harmonic amplitudes, $a_{k}$, to the amplitude of the fundamental frequency, $a_{1}$, (Miller \& Liggieri 2009; Loglio et al. 2004).

$$
T H D=\frac{\sqrt{\sum_{k=2}^{n} a_{k}^{2}}}{a_{1}}
$$

THD values were calculated for original and smoothed stress and strain waveforms measured at different applied stress levels (Table 5.1). THD calculated for input stress signals is below $1 \%$, and considerably smaller compared to the THD of the resulting strain signals. THD of the strain signals are between $9.1403 \%$ and 17.6911 $\%$, and THD is increasing with increasing levels of applied stresses as the material is being probed more into the nonlinear regime. The difference between the THD of original and smoothed signals are negligible $(<0.3 \%$ in all cases) suggesting that the rich nonlinear characteristics of the samples have not been lost due to the applied smoothing spline fit. 
Table 5.1 Total harmonic distortion calculated for stress and strain signals.

\begin{tabular}{|c|c|c|c|}
\hline \multicolumn{4}{|c|}{ Total Harmonic Distortion (THD) / \% } \\
\hline Applied stress & $\mathbf{1 0 0}$ Pa & $\mathbf{5 0 0}$ Pa & $\mathbf{1 5 0 0}$ Pa \\
\hline THD of original stress & 0.1896 & 0.5542 & 0.5077 \\
\hline THD of smoothed stress & 0.1710 & 0.4372 & 0.3949 \\
\hline THD difference in stress & 0.0186 & 0.1169 & 0.1127 \\
\hline THD of original strain & 9.1403 & 12.9656 & 17.6911 \\
\hline THD of smoothed strain & 9.1098 & 12.7027 & 17.4499 \\
\hline THD difference in strain & 0.0305 & 0.2629 & 0.2412 \\
\hline \hline
\end{tabular}

THD analysis of the data sets further validates that the frequency spectra of the smoothed waveforms are identical to their original waveforms providing evidence that the process of smoothing does not eliminate the essential information originating from the nonlinear response of the sample. Henceforth, smoothed stress and strain waveforms were used as the basis of the Lissajous-Bowditch curves and calculation of nonlinear compliances.

Figure 5.12 shows a comparison of Lissajous-Bowditch curves constructed from original and smoothed datasets. 

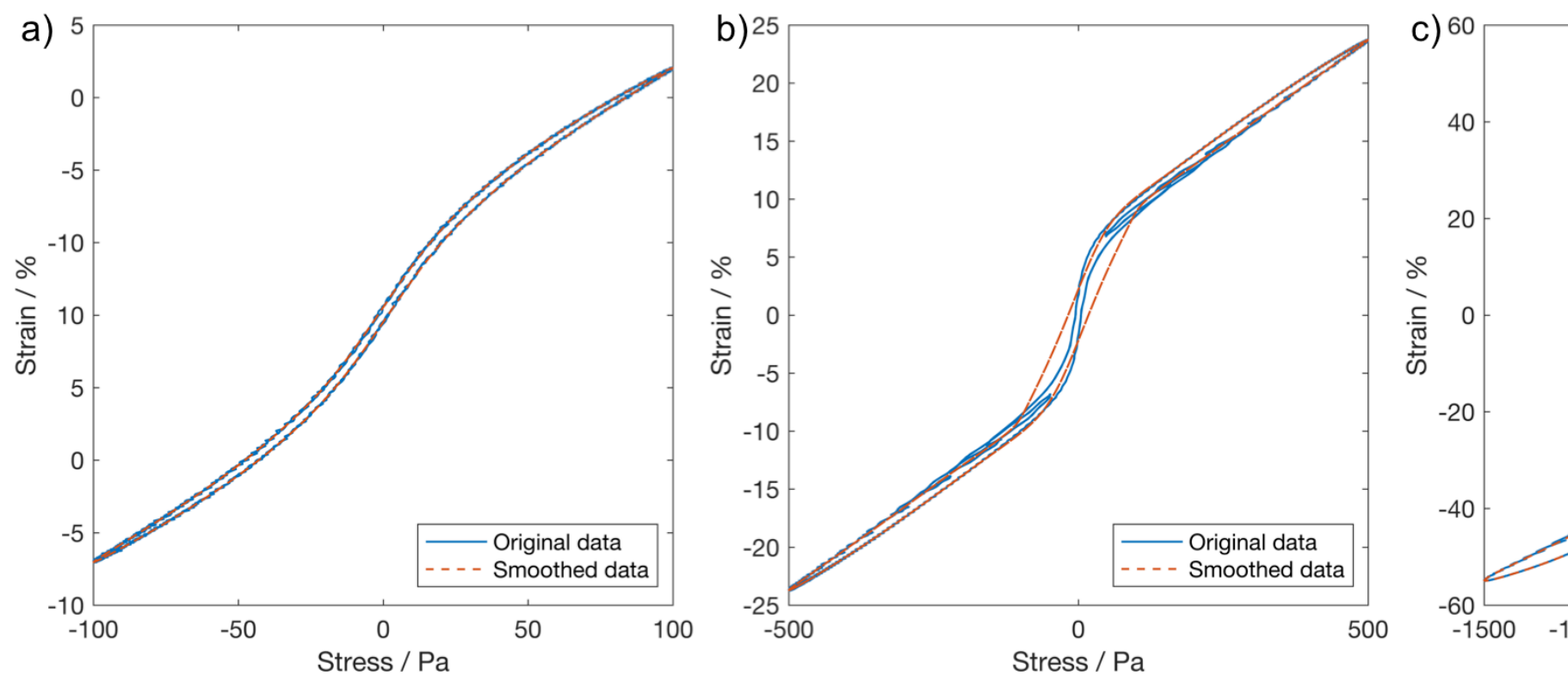

Figure 5.12 Comparison of Lissajous-Bowditch curves using original (blue solid line) and smoothed (red dotted line) datasets for input stress lev 
At relatively low levels of stress (Figure 5.12a), the Lissajous-Bowditch curves appear identical. Figures $5.12 \mathrm{~b}$ and $5.12 \mathrm{c}$ show that the ringing encountered in the original waveforms introduces secondary loops in the corresponding LissajousBowditch curves. These secondary loops are eliminated by the process of smoothing. Furthermore, at these relatively large stress levels, inspection of the original and smoothed curves shows profound differences around the region pertaining to the minimum stress. Thus, any calculated values of $J_{M}^{\prime}$ would be highly sensitive to ringing artefacts.

\subsubsection{Structural changes in LAOStress}

Figure 5.13 shows the results of the alternating LAOStress and SAOS measurements. The applied stress levels (Figure 5.13a), and the resulting strain (Figure 5.13b) is displayed as a function of time. The time period for which each stress is applied depends on the duration of the measurement to reach a steady state (as shown in Chapter 4.4.1). Figure 5.13c and Figure 5.13d display the calculated values of nonlinear and linear compliances, respectively. The nonlinear compliances, $J_{M}^{\prime}$ and $J_{L}^{\prime}$ gradually decrease with increasing levels of stress and strain, and $J_{M}^{\prime}$ dominates over $J_{L}^{\prime}$ over the entire range of stress levels studied. 


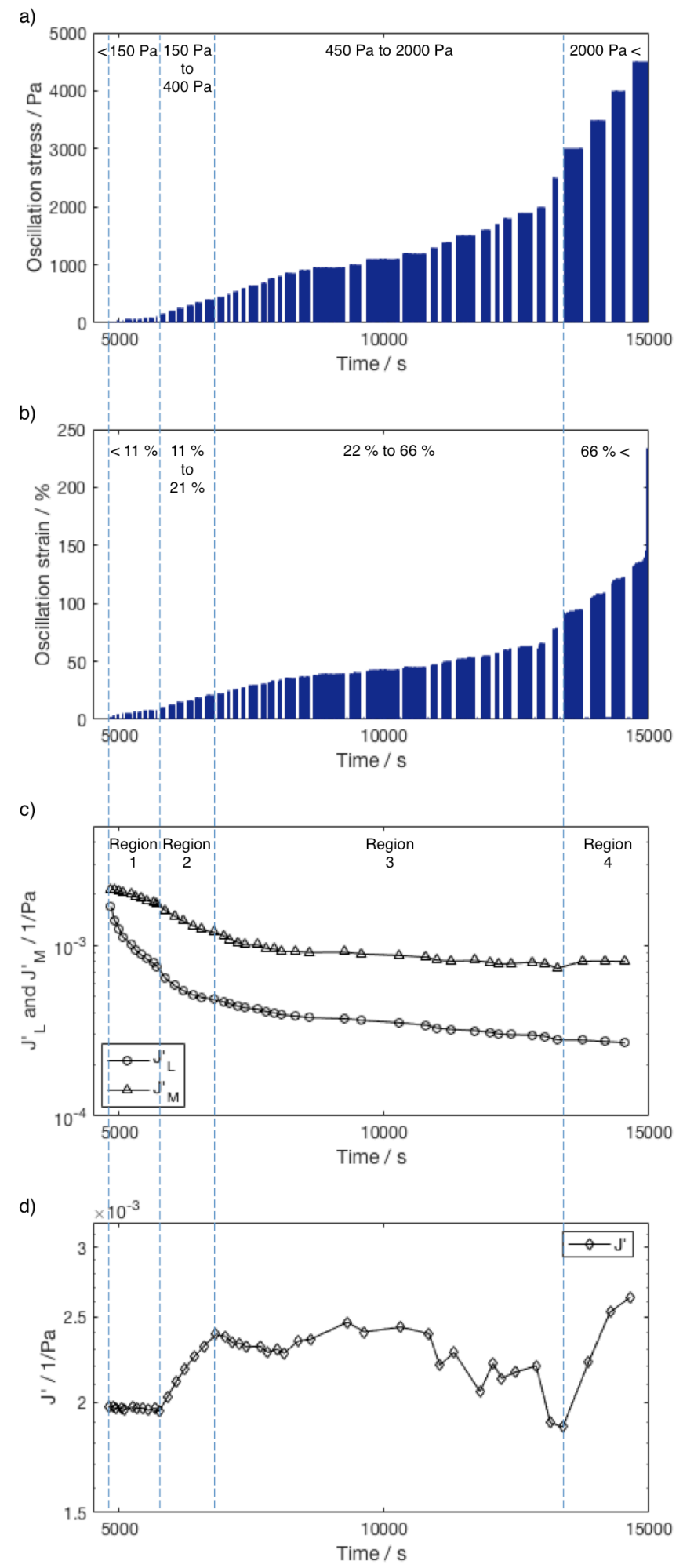

Figure 5.13 Result of alternating SAOS and LAOStress measurements on a clot formed by the addition of 4 $\mathrm{mg} / \mathrm{ml}$ fibrinogen and $0.1 \mathrm{NIH} / \mathrm{ml}$ thrombin; (a) the applied stress versus time profile, (b) the resulting strain response versus time profile, (c) large-stress and minimum-stress elastic compliances (as measured by LAOStress), and (d) linear elastic compliance (as measured by SAOS). The results reveal several nonlinear viscoelastic regions (numbered 1-4, as illustrated by the dashed lines). 
Table 5.2 shows the characteristics of the identified nonlinear regions in terms of changes in the linear and nonlinear compliances. In Region 1 , both $J_{M}^{\prime}$ and $J_{L}^{\prime}$ decreased with increasing levels of stress, whilst the linear compliance is constant. In Region 2 the linear elastic compliance gradually increased following the application of increasing levels of LAOStress, whilst $J_{M}^{\prime}$ and $J_{L}^{\prime}$ continued to decrease. The behaviour encountered in Region 3 is similar to Region 1 insofar that the linear elastic compliance is independent of the stress applied in the LAOStress measurement, and $J_{M}^{\prime}$ and $J_{L}^{\prime}$ are decreasing. In Region 4, further increases in the applied levels of stress lead to fracture of the sample network causing increase in both linear and nonlinear compliances, and the rheometer upper plate to overspeed.

Table 5.2 Summary of the different characteristics of each nonlinear region in terms of changes in linear and nonlinear compliances. $\uparrow$ represents increase, $\downarrow$ represents decrease, whilst $=$ represents unchanging values.

\begin{tabular}{|c|c|c|c|c|}
\hline & Region 1 & Region 2 & Region 3 & Region 4 \\
\hline$J^{\prime}$ & $=$ & $\uparrow$ & $=$ & $\uparrow$ \\
\hline$J_{M}^{\prime}$ & $\downarrow$ & $\downarrow$ & $\downarrow$ & $\uparrow$ \\
\hline$J_{L}^{\prime}$ & $\downarrow$ & $\downarrow$ & $\downarrow$ & $\uparrow$ \\
\hline
\end{tabular}

\subsubsection{SEM imaging of fibrin clots probed in LAOStress measurements}

The microstructure of the fibrin clots, that had been subjected to several different stress levels in LAOStress, were studied using a scanning electron microscope in order to investigate the formed structure in the previously established nonlinear viscoelastic regions. Figure 5.14 shows SEM images of fully formed clots that had been previously subjected to stress levels of $5 \mathrm{~Pa}$ (linear viscoelastic region, LVR), $100 \mathrm{~Pa}$ (Region 1), $300 \mathrm{~Pa}$ (Region 2), 600Pa (Region 3), and $4000 \mathrm{~Pa}$ (Region 4). The structure of the fibrin clot in Region 1 was very similar to the fibrin clot tested in LVR (control sample). In Region 2 the fibres were stretched and aligned to the shearing direction, creating a more densely packed network. Region 3 showed similar characteristics to Region 2, moreover some localised fracture of the fibres can also be observed, as the network rearranged. SEM images of Region 4 showed damaged and broken individual fibres, with clumping of the network, eventually leading to complete fracture of the sample. 

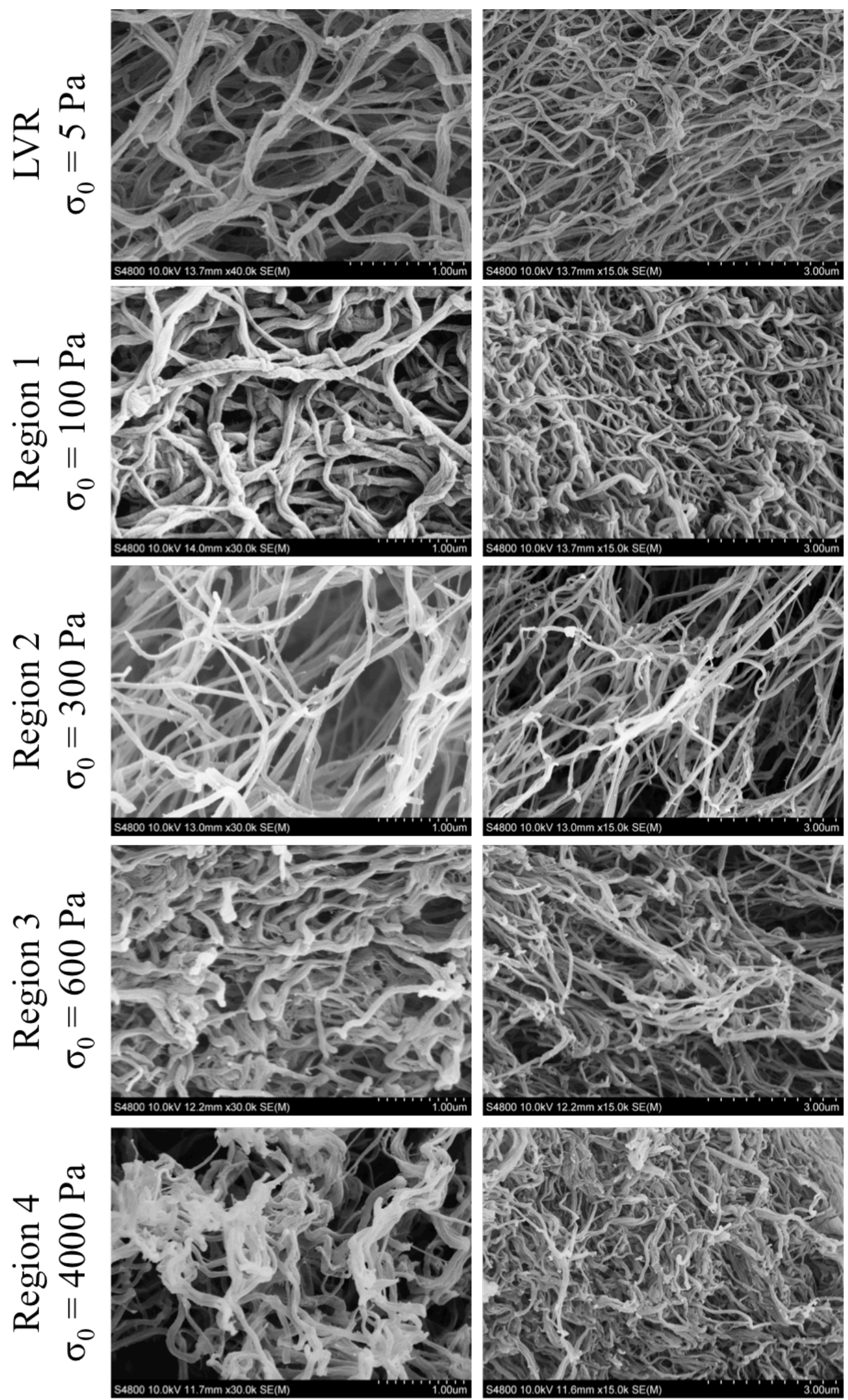

Figure 5.14 SEM images of fibrin clot structures in the established viscoelastic regions. 


\subsubsection{Structural changes during LAOStress in clots formed with different thrombin concentrations}

All four nonlinear regions as observed previously were also present for clots formed with different thrombin levels (Figure 5.15 and Figure 5.16). By investigating the clots formed with different thrombin concentrations, it was found that both the linear and nonlinear elastic compliances display different characteristics in terms of their variation in response to increasing levels of stress. Figure 5.15 and Figure 5.16 shows the result of alternating SAOS and LAOStress measurements conducted on clots formed with relatively low levels of thrombin, $0.06 \mathrm{NIH} / \mathrm{ml}$ and $0.02 \mathrm{NIH} / \mathrm{ml}$, respectively; compared to the result shown in the previous section (see Figure 5.13 in Chapter 5.4.4). In contrast to the structural changes of the clot formed with higher thrombin concentration $(0.1 \mathrm{NIH} / \mathrm{ml})$, the fibrin clot formed with lower thrombin level $(0.02 \mathrm{NIH} / \mathrm{ml})$ showed a different pattern in terms of changes in linear elastic compliances as the stress increases. Before Region 4, there is a decrease in linear elastic compliance for the higher thrombin concentration, while it gradually increases in the case of the lower thrombin content. 
a)
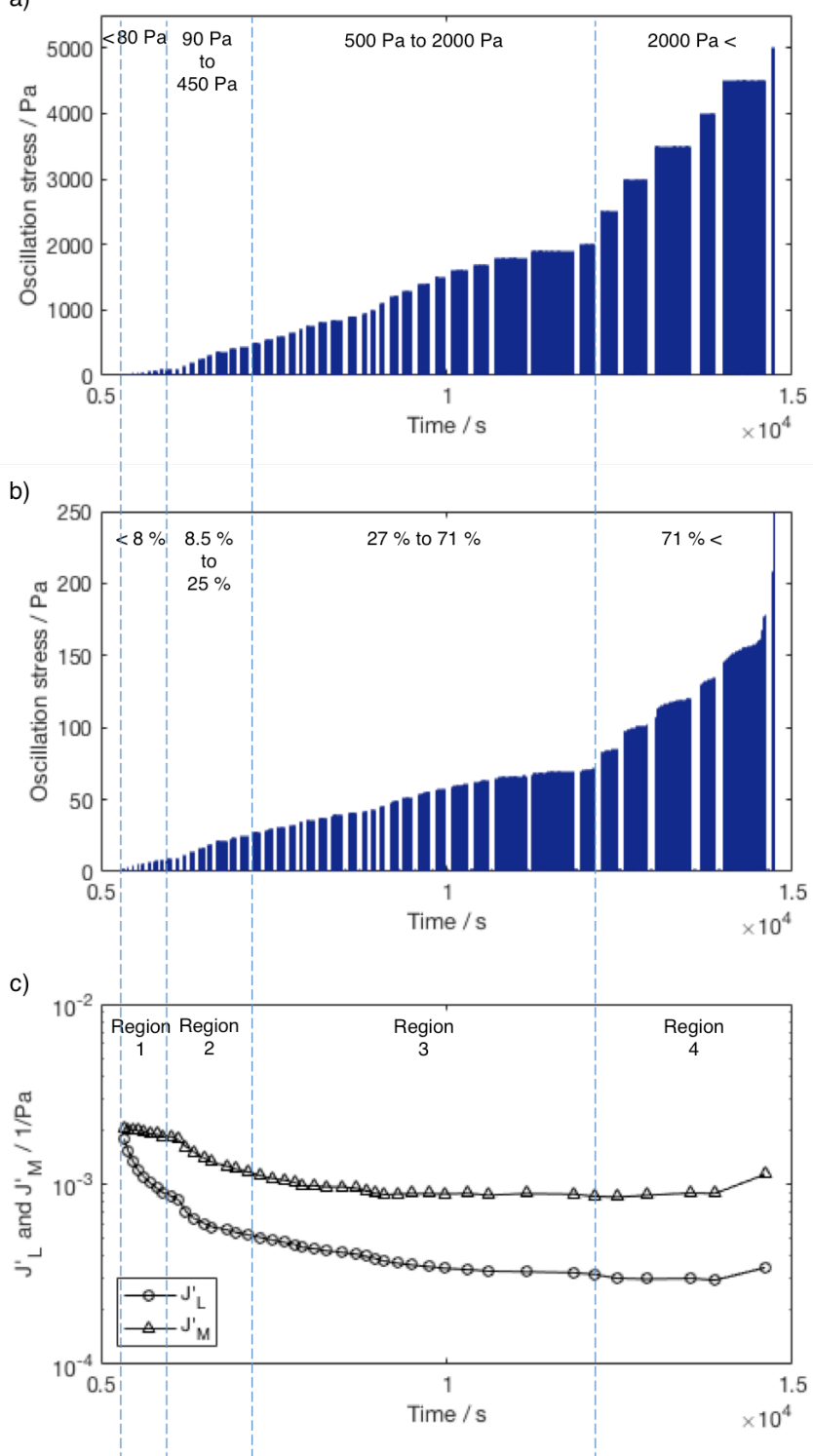

d)

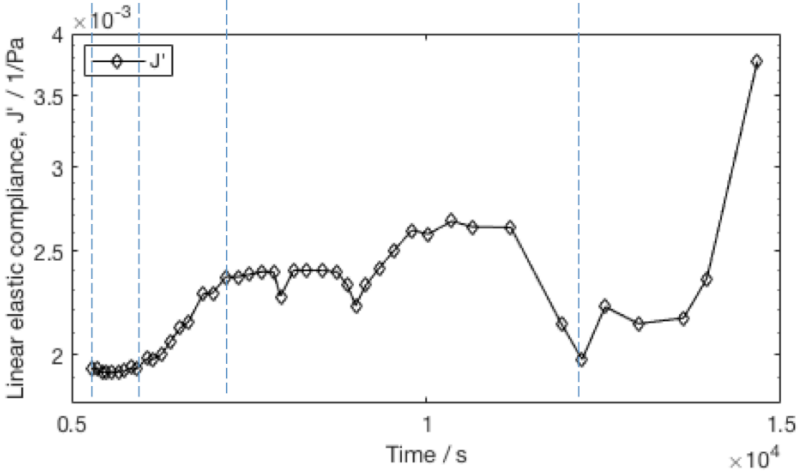

Figure 5.15 Result of alternating SAOS and LAOStress measurements on a clot formed by the addition of 4 $\mathrm{mg} / \mathrm{ml}$ fibrinogen and $0.06 \mathrm{NIH} / \mathrm{ml}$ thrombin; (a) the applied stress versus time profile, (b) the resulting strain response versus time profile, (c) large-stress and minimum-stress elastic compliances (as measured by LAOStress), and (d) linear elastic compliance (as measured by SAOS). The results reveal several nonlinear viscoelastic regions (numbered 1-4, as illustrated by the dashed lines). 

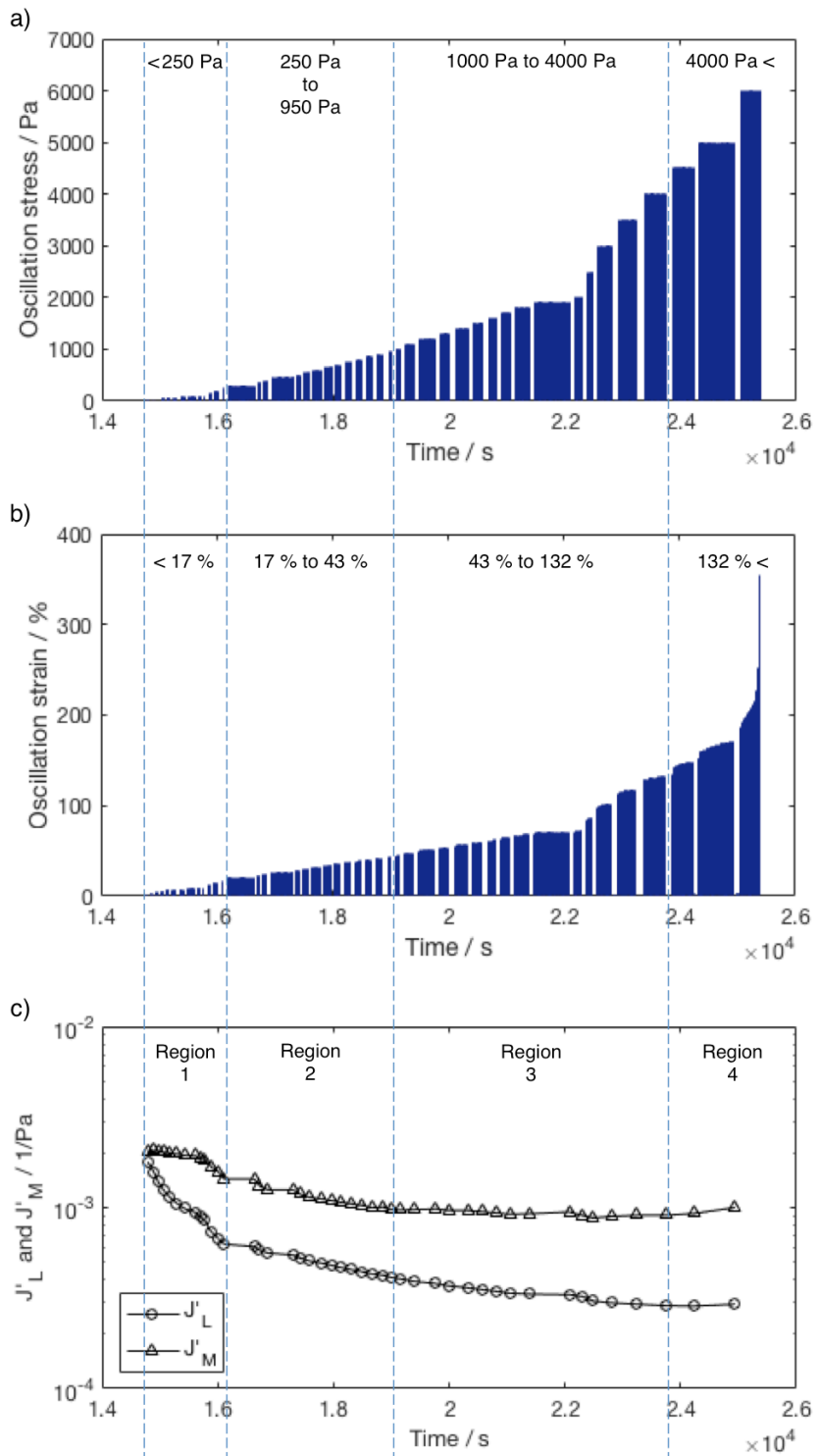

d)

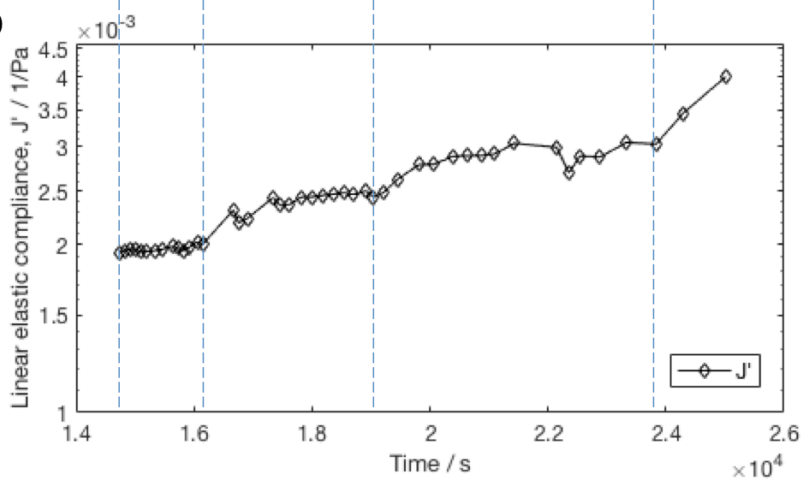

Figure 5.16 Result of alternating SAOS and LAOStress measurements on a clot formed by the addition of 4 $\mathrm{mg} / \mathrm{ml}$ fibrinogen and $0.02 \mathrm{NIH} / \mathrm{ml}$ thrombin; (a) the applied stress versus time profile, (b) the resulting strain response versus time profile, (c) large-stress and minimum-stress elastic compliances (as measured by LAOStress), and (d) linear elastic compliance (as measured by SAOS). The results reveal several nonlinear viscoelastic regions (numbered 1-4, as illustrated by the dashed lines). 
Figure 5.17 and Figure 5.18 summarise the detected viscoelastic regions in term of stress and strain ranges for all investigated thrombin concentrations. Experiments were repeated three times at each thrombin concentration, and the displayed error bars represent standard deviations. It can be seen that the regions have shifted as different thrombin concentrations were used.

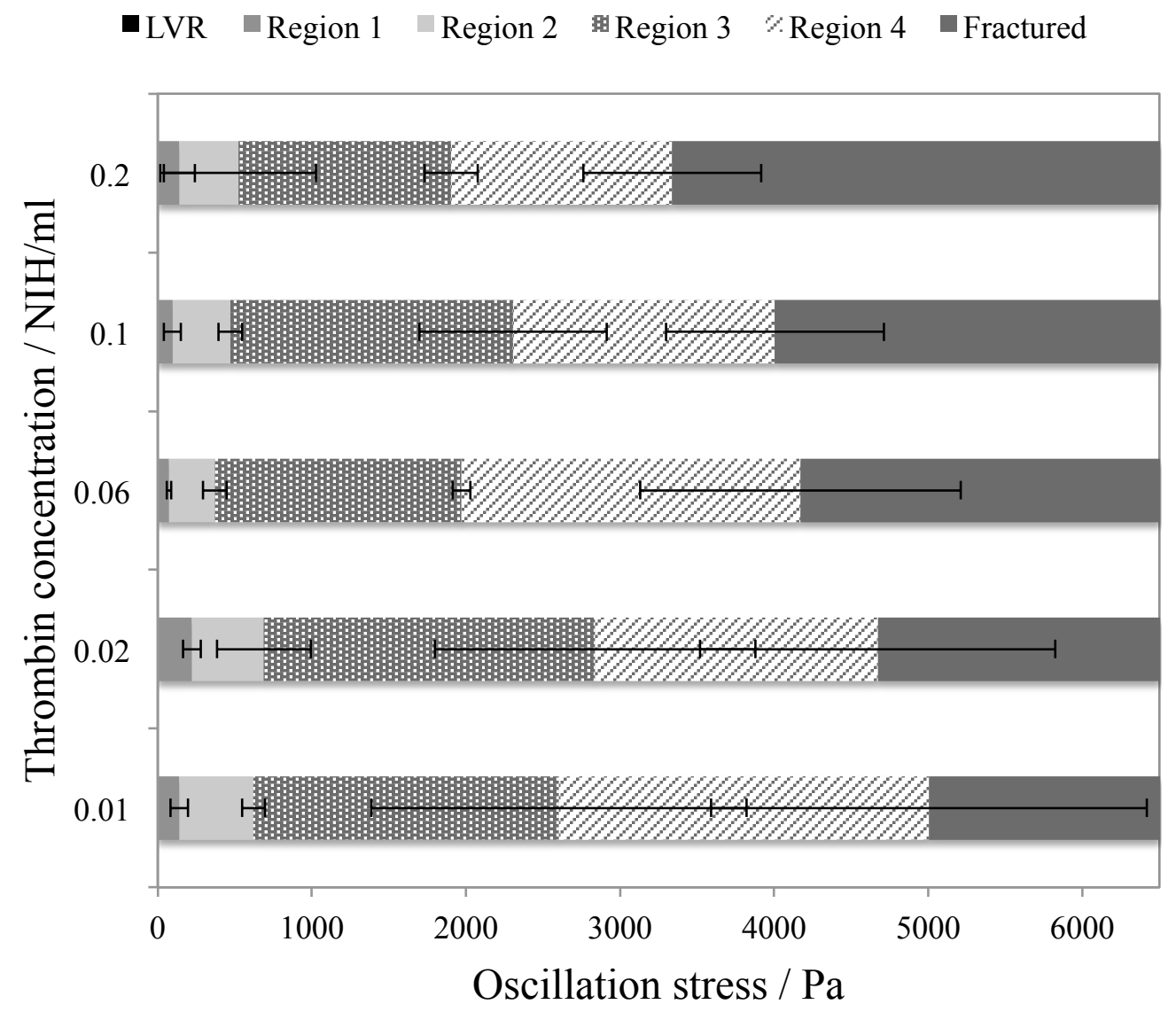

Figure 5.17 Nonlinear regions observed for fibrin clots formed with different thrombin concentrations in terms of applied oscillatory stress levels. 


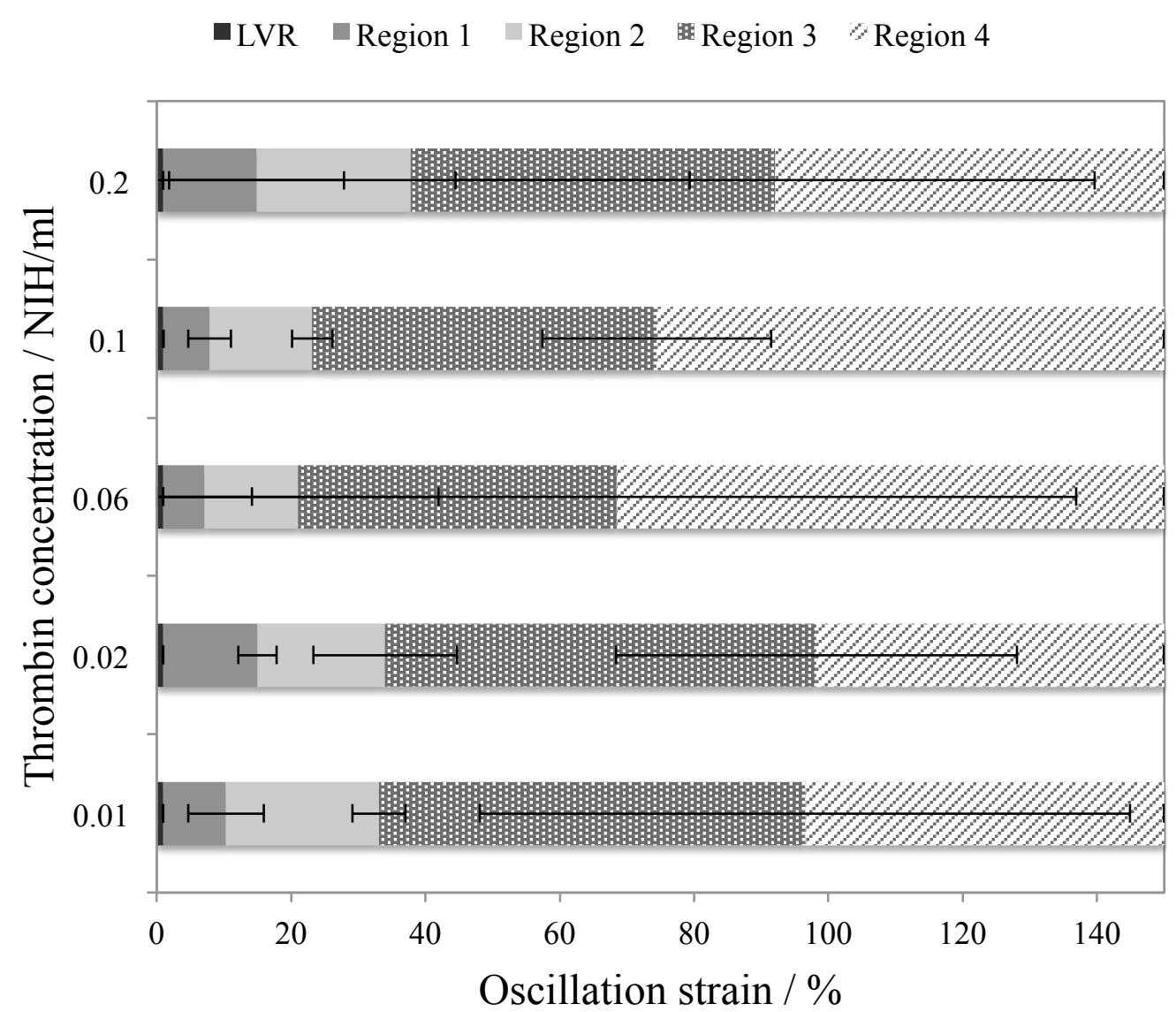

Figure 5.18 Nonlinear regions observed for fibrin clots with different thrombin concentrations in terms of resultant oscillatory strain levels.

\subsubsection{Effect of thrombin concentration of the fracture stress of fully formed fibrin clots}

Figure 5.19 shows the effect of different thrombin levels on the eventual fracture of the fully formed fibrin clots. Experiments were repeated three times at each thrombin concentration, and the displayed error bars represent standard deviations. The addition of increasing levels of thrombin produced clots that displayed decreasing values of fracture stress. 


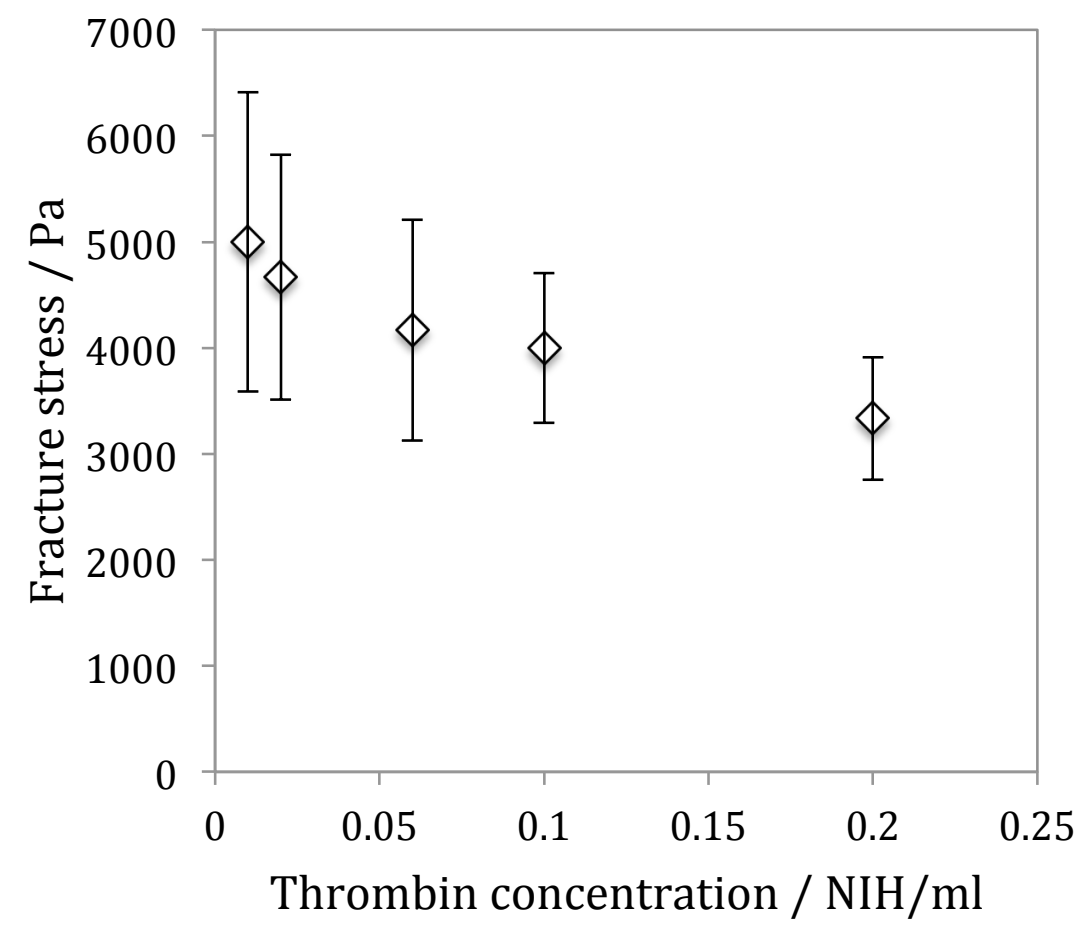

Figure 5.19 The measured fracture stress as a function of thrombin concentration.

\subsubsection{LAOStress properties of fibrin clots formed under CSPS}

Fibrin clots were formed under CSPS, in order to study the effect of unidirectional stress on the nonlinear viscoelastic properties. Previously, effect of unidirectional stress values $(0.047 \mathrm{~Pa}$ to $0.4 \mathrm{~Pa})$ on whole blood clots and fibrin clots with 10 $\mathrm{mg} / \mathrm{ml}$ fibrinogen concentration was studied by Badiei et al. (Badiei et al. 2015). Herein, the effect of lower unidirectional stresses $(0.005 \mathrm{~Pa}$ and $0.01 \mathrm{~Pa})$ on fibrin clots formed with a physiologically relevant fibrinogen concentration $(4 \mathrm{mg} / \mathrm{ml})$ was studied. Due to the lower level of fibrinogen concentration $(4 \mathrm{mg} / \mathrm{ml})$ in the model system, it was not possible to study effects of higher unidirectional stresses, as those resulted in complete disruption of the structure during clot formation as evidenced by the inability of the rheometer to monitor gradual increase in complex modulus during gelation.

Figure 5.20 and 5.21 display the results of the alternating LAOStress and SAOS measurements for clots formed under CSPS with unidirectional stress of $0.005 \mathrm{~Pa}$ and $0.01 \mathrm{~Pa}$, respectively. The nonlinear behaviour of these clots is similar to the clot 
with same thrombin concentration, formed under SAOS insofar that the different nonlinear regions share the same characteristics (Figure 5.13). Figure 5.13c, Figure $5.20 \mathrm{c}$ and $5.21 \mathrm{c}$ show that nonlinear compliances, $J_{M}^{\prime}$ and $J_{L}^{\prime}$ gradually decrease with increasing levels of stress and strain, and $J_{M}^{\prime}$ dominates over $J_{L}^{\prime}$ over the entire range of stress levels studied. Whilst, as shown in Figure 5.13d, Figure 5.20d and 5.21d, there is a decrease in $J^{\prime}$ in the end of Region 3. 

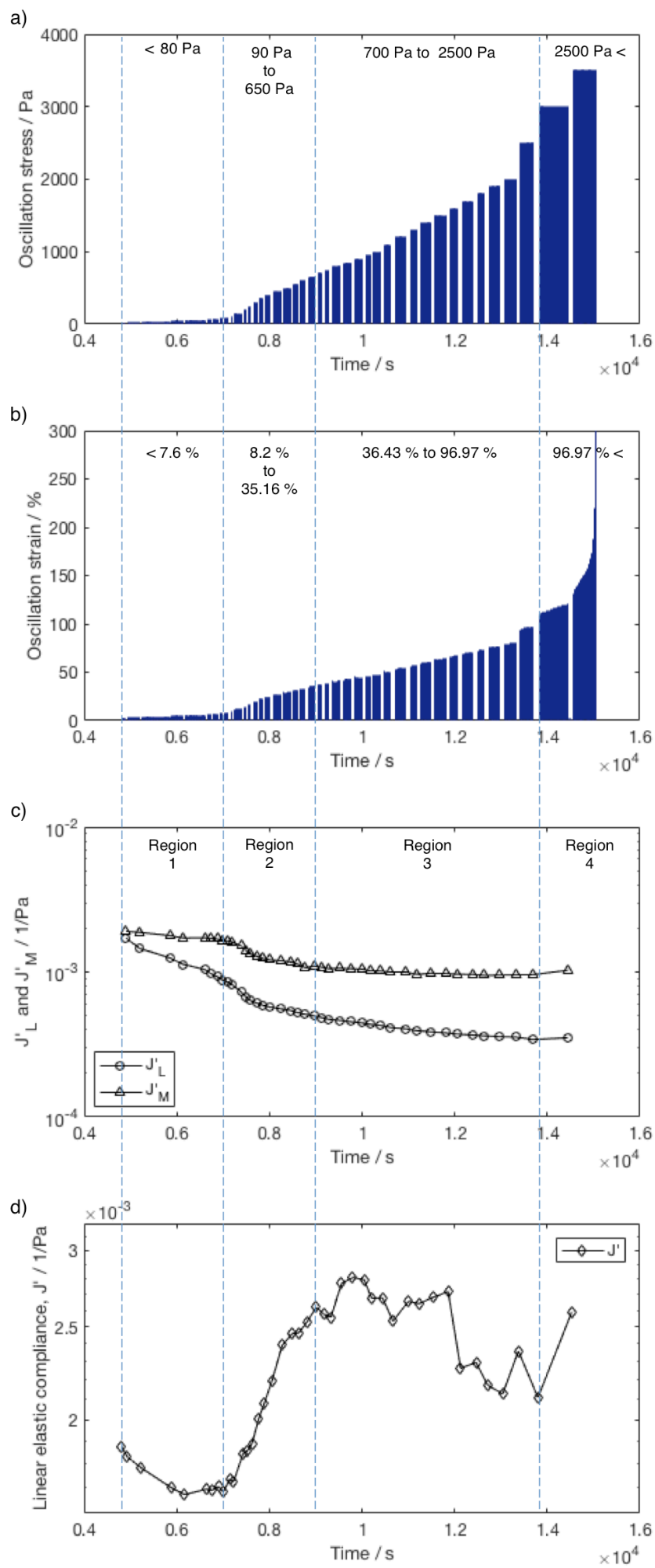

Figure 5.20 Result of alternating SAOS and LAOStress measurements on a clot formed under CSPS $\left(\boldsymbol{\sigma}_{\boldsymbol{s}}=0.005\right.$ $\mathrm{Pa}$ ) by the addition of $4 \mathrm{mg} / \mathrm{ml}$ fibrinogen and $0.1 \mathrm{NIH} / \mathrm{ml}$ thrombin; (a) the applied stress versus time profile, (b) the resulting strain response versus time profile, (c) large-stress and minimum-stress elastic compliances (as measured by LAOStress), and (d) linear elastic compliance (as measured by SAOS). The results reveal several nonlinear viscoelastic regions (numbered 1-4, as illustrated by the dashed lines). 


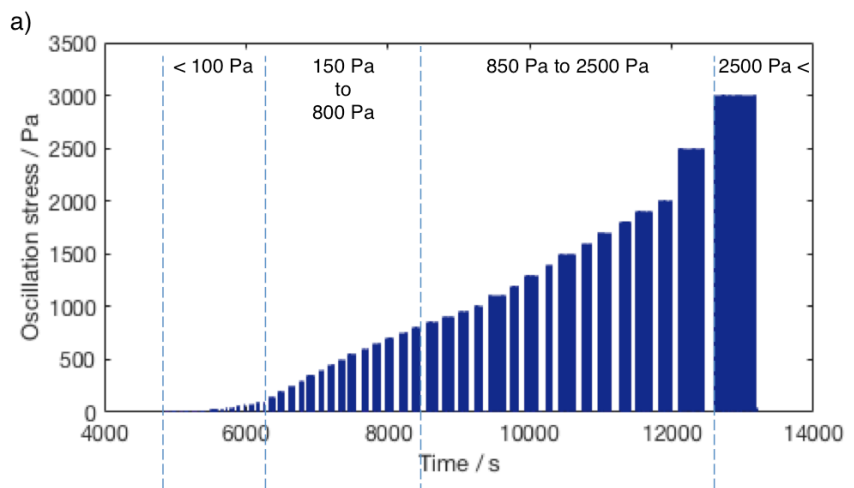

b)
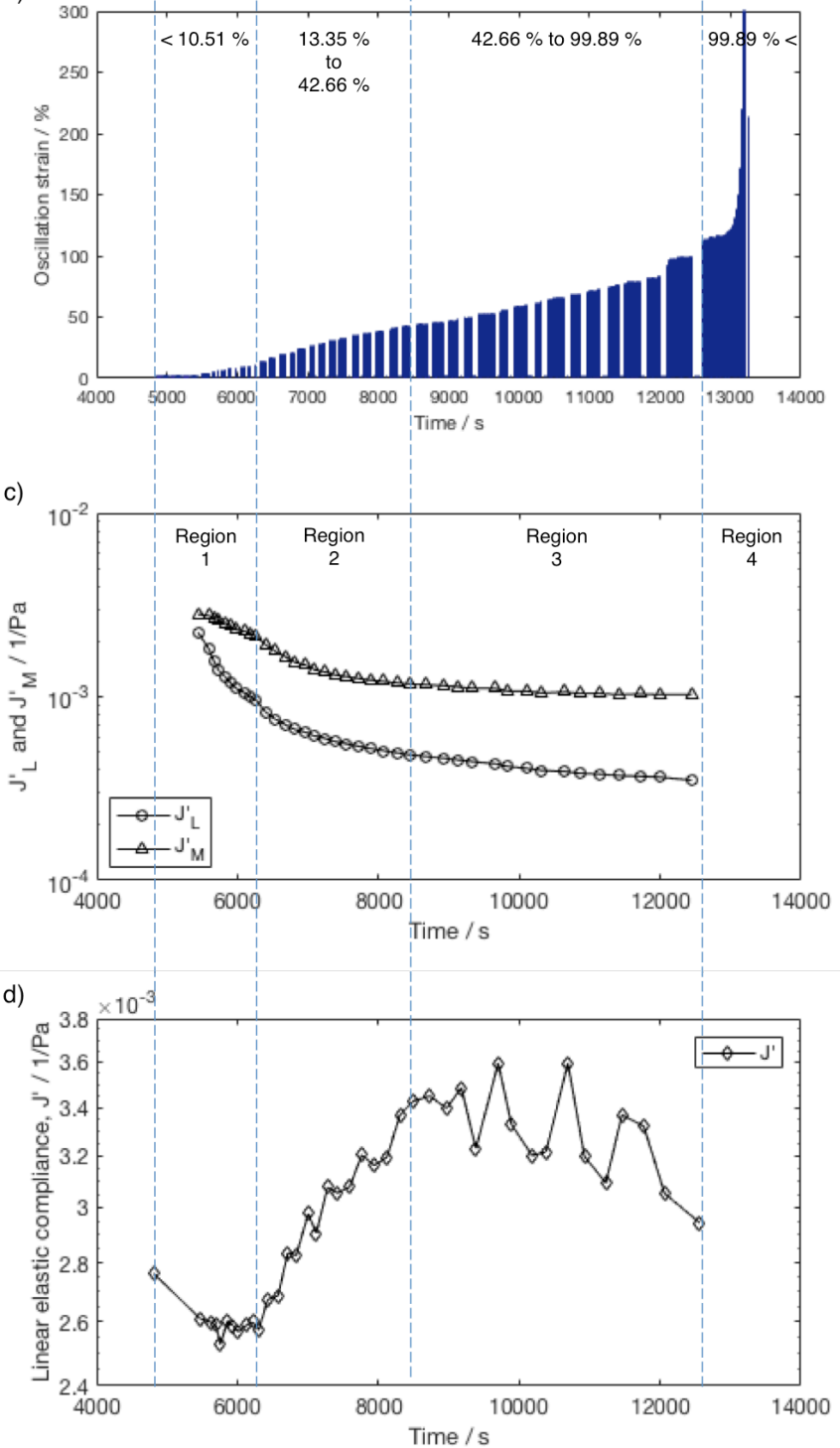

Figure 5.21 Result of alternating SAOS and LAOStress measurements on a clot formed under CSPS $\left(\boldsymbol{\sigma}_{\boldsymbol{s}}=0.01\right.$ $\mathrm{Pa}$ ) by the addition of $4 \mathrm{mg} / \mathrm{ml}$ fibrinogen and $0.1 \mathrm{NIH} / \mathrm{ml}$ thrombin; (a) the applied stress versus time profile, (b) the resulting strain response versus time profile, (c) large-stress and minimum-stress elastic compliances (as measured by LAOStress), and (d) linear elastic compliance (as measured by SAOS). The results reveal several nonlinear viscoelastic regions (numbered 1-4, as illustrated by the dashed lines). 
All four aforementioned regions (see Chapter 5.4.3) were detected in the case of the clots formed under CSPS. Experiments were repeated three times at each unidirectional stress level, and the displayed error bars represent standard deviations. Forming clots under CSPS only slightly affected the detected nonlinear regions (Figure 5.22 and 5.23). The application of increasing unidirectional stress levels led to narrower stress ranges of Region 4, as the end of Region 4 shifted the lower stress levels. Consequently, the eventual fracture stresses shifted to slightly lower levels of stresses with increasing unidirectional stress in the CSPS step.

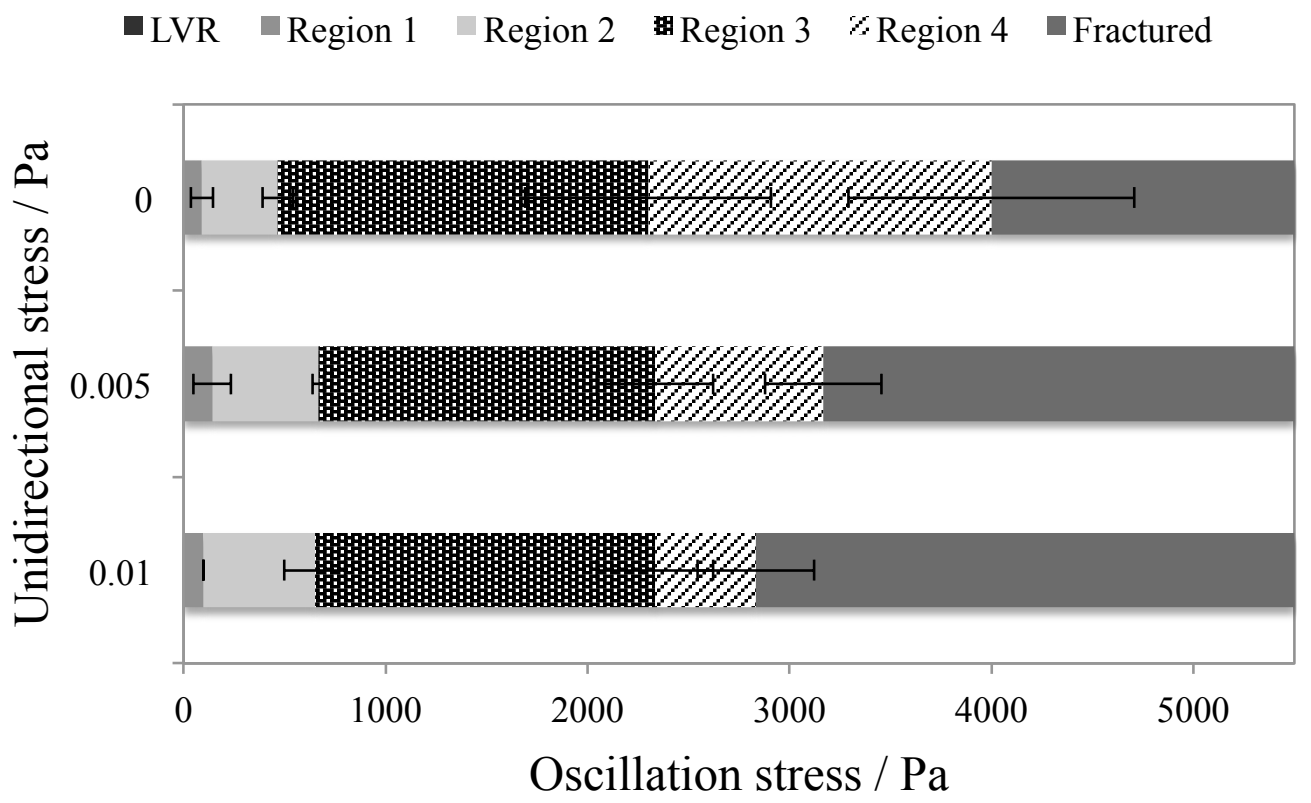

Figure 5.22 Nonlinear regions - in terms of oscillatory stress ranges - observed for fibrin clots subjected to different levels of unidirectional stress. 


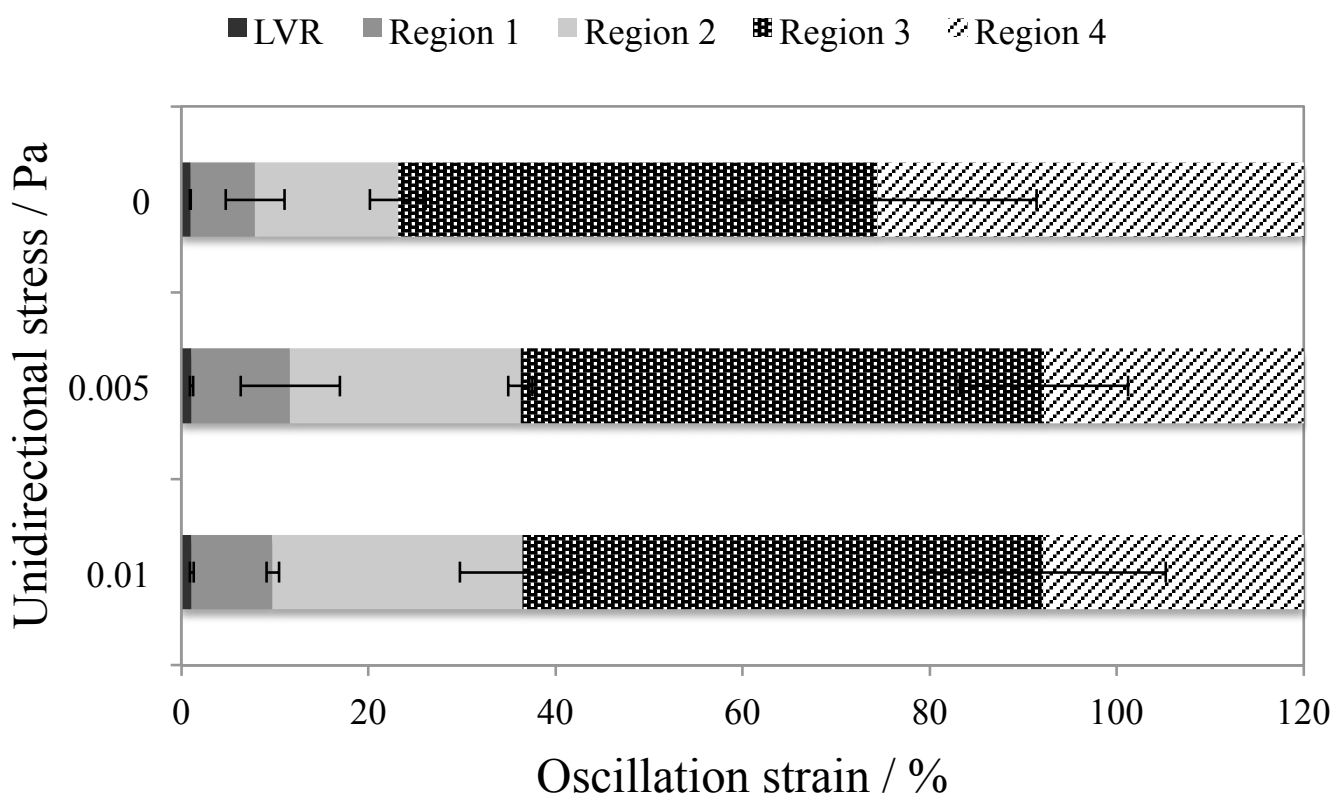

Figure 5.23 Nonlinear regions - in terms of oscillatory stress ranges - observed for fibrin clots subjected to different levels of unidirectional stress.

\subsection{Discussion}

The LAOStress measurements of fibrin clots at relatively large applied stresses were polluted by a source of ringing which manifests in both the applied stress and resulting strain. The ringing was eliminated by fitting a smoothing spline to both the stress and strain data sets. Fourier transformation analysis of the original and smoothed waveforms showed that this is an appropriate approach to eliminate the ringing, as the essential harmonic information is retained. The degree of system nonlinearity, as calculated in terms of THD, was similar in raw and smoothed waveforms therefore providing further evidence that meaningful nonlinear information was not lost during smoothing of the waveforms. Therefore, the smoothed waveforms were used to calculate the values of nonlinear compliances from the LAOStress measurements.

The ringing in the LAOStress waveforms was analysed and compared to a similar analysis of inertio-elastic ringing using data obtained from a creep measurement on the same sample. The analysis showed that the differential moduli obtained from analysis of the ringing in the creep and LAOStress were of similar magnitude 
suggesting that this ringing provides additional information on the rheological properties of the material. This also provides evidence that the source of ringing seen in the LAOStress measurement is the same as that seen in the creep measurement and is likely to be a coupling of inertial and elastic forces. The differential moduli further quantifies the nonlinear behaviour as shown by an increase in the differential moduli with increasing stress levels, confirming a stress stiffening response.

Structural changes following the subjection to elevated stresses were studied by the application of alternating SAOS and LAOStress steps to the mature fibrin clot. The gradual decrease in the nonlinear compliances, $J_{M}^{\prime}$ and $J_{L}^{\prime}$, with increasing levels of stress and strain indicated stress stiffening of the fibrin clot formed with $0.1 \mathrm{NIH} / \mathrm{ml}$ thrombin. Intracycle stress stiffening was also captured in individual LAOStress measurements at discrete levels of stress by a dominance of $J_{M}^{\prime}$ over $J_{L}^{\prime}$ over the entire range of stress levels studied. This behaviour is expected given that individual fibrin fibres' strain stiffens and is highly extensible (Hudson et al. 2010; Liu et al. 2010; Piechocka et al. 2010). The results are similar to the LAOS controlled strain studies of van Kempen et al. that reported intra-cycle stiffening and softening during continuous application of large strain deformations to fibrin clots (van Kempen et al. 2015). However, an advantage of the LAOStress measurements employed herein is that the fibrin clot is allowed to reach the steady state under a fixed level of oscillatory stress. This is more physiologically relevant insofar that the stress is imposed on the clot (Colace et al. 2012; Kroll et al. 1996).

Observation of the nonlinear and linear viscoelastic behaviour of the sample (in Figure 5.13c and 5.13d, respectively, in Chapter 5.4.3) reveals four distinct regions within the nonlinear range of the material. Therefore LAOStress revealed more detailed structural information than LAOStrain (see Chapter 4) where only three different regions were identified. The stress and strain ranges measured for the different regions in LAOStress and LAOStrain differ, which might be expected as results in LAOStress and LAOStrain are not interchangeable, and may therefore provide distinct information (Ewoldt 2009). In Region $1\left(\sigma_{0}<150 \mathrm{~Pa}\right)$, the sample shows increasing tendency to stress stiffen as evidenced by an increase in the difference between $J_{M}^{\prime}$ and $J_{L}^{\prime}$ with increasing levels of stress. The sample returns to 
its original linear viscoelastic behaviour following a decrease in applied stress indicating full recovery of the structure. This behaviour could be expected as it has been shown before that individual fibrin fibres can be stretched threefold before breaking (Brown et al. 2009). In Region $2\left(150<\sigma_{0}<400 \mathrm{~Pa}\right)$, the linear elastic compliance gradually increased following the application of increasing levels of LAOStress, indicating irreversible structural rearrangements. This stress-softening, which is also evident by close inspection of the curvature of the oscillatory strain response in the LAOStress experiments, is likely to be due to yielding of branching points leading to a slackening of the network (Liu et al. 2006). The behaviour encountered in Region $3\left(450<\sigma_{0}<2000 \mathrm{~Pa}\right)$ is similar to Region 1 insofar that the sample returns to a level of linear elastic compliance that is independent of the stress applied in the LAOStress measurement. This finding is similar to the studies of Piechocka et al. and Munster et al., which showed that the differential modulus is independent of values of pre-stress within a specific stress regime (Piechocka et al. 2010; Munster et al. 2013). In Region 3, the structure of the sample has been permanently re-arranged by the stresses encountered in Region 2, resulting in a sample that has the ability to resist structural changes when exposed to further increases in stress. Furthermore, in the latter stages of Region 3 (i.e., at long times), there is generally a decrease in linear elastic compliance following the application of increasing levels of LAOStress, indicating that effects due to ageing of the sample (possibly due to Factor XIII mediated cross-linking) are greater than any stress induced structural damage. In Region $4\left(\sigma_{0}>2000 \mathrm{~Pa}\right)$, the ability of the sample to resist structural changes at increasing levels of stress diminishes as evidenced by an increase in linear elastic compliance. Further increases in the applied levels of stress lead to fracture of the sample network causing the rheometer upper plate to overspeed.

The SEM images of the clots that had been subjected to LAOS were highly complementary to the rheometry results and explained some of the mechanisms of structural changes. The images revealed differences in structure in each of the nonlinear regions. The structure in Region 1 was very similar to the control sample (fibrin clot tested by the application of SAOS). In Region 2 and Region 3 the fibres were aligned to the shearing direction and the network appeared to be more densely 
packed, with some localised fracture of the fibres, as the network rearranged. SEM images of Region 4 showed damaged and broken individual fibres, with clumping of the network, eventually leading to complete fracture of the sample.

Previous studies have shown that by forming fibrin clots with different thrombin concentrations results in different incipient and mature clot microstructures, with relatively low level of thrombin producing clots with a more open structure with thick fibres, and relatively high level of thrombin producing clots with a more dense structure consisting of thin fibres (Weisel 2007; Wolberg 2007; Hawkins et al. 2010; Curtis et al. 2013; Weisel \& Litvinov 2013b). LAOStress properties of fibrin clots with different thrombin concentrations, and henceforth different microstructures were studied, and a shift in some of the nonlinear regions to different magnitudes of applied stress was observed. In the case of the fibrin clots formed with lower thrombin concentrations, the end of Region 3 and Region 4 shifted to higher stress levels. Consequently, larger eventual fracture stress values were measured for the lower thrombin levels, suggesting that clots formed with lower thrombin concentrations have an ability to adapt and build up more resistance to the applied stress.

In the samples formed with higher thrombin concentrations a decrease in linear elastic compliance can be observed in Region 3 - possibly due to bundling of the fibres or aging - at longer times, prior to Region 4. This decrease in linear elastic compliance cannot be seen in the case of clots formed with relatively low $(0.02$ $\mathrm{NIH} / \mathrm{ml}$ ) thrombin concentration (Figure 5.16 in Chapter 5.4.5), suggesting an interplay between the effect of bundling or aging and the rate of stress induced damage which is dependent on the clot microstructure. Fibrin clots formed with high thrombin concentration consist of thinner fibres and more branch points, hence form a more dense network. Whilst, fibrin clots formed by the addition of low thrombin concentration consists of thicker fibres with fewer branch points, and has a more open clot structure. As the dense clots (i.e. high thrombin concentration) experience levels of stresses in Region 3, their fibres are bundling, which might lead to a structure similar to what can be seen in the initially more open clots (i.e. low thrombin concentration), hence causing the observed decrease in the linear elastic compliance (i.e. stress stiffening behaviour) in Region 3. However, since the fibres 
are already stretched, irreversible deformation (i.e. localised fracture) takes place in Region 4, which results in the complete fracture of the clot. In contrast, in the case of clots formed with low thrombin, the fibrin fibres are thicker, which is why the fibres might be stretched more, causing the observed regions to shift to higher levels of stresses. However, in this case the bundling of the fibres in Region 3 might not result in a more beneficial structure. Assuming that the diameter of the stretched fibres is similar for both thicker fibres and thinner fibres would suggest that fewer fibre bundles form in the case of low thrombin concentration, as those clots initially consist of thicker but fewer fibres in comparison to the more dense clots formed with high thrombin concentration. This network structure in Region 3, consisting of fewer fibre bundles could not withstand further increase in stress. Therefore, the network weakens (i.e. stress softening behaviour) in the end of Region 3, leading to a gradual increase in linear elastic compliance in Region 4.

Mature fibrin clots formed with $0.1 \mathrm{NIH} / \mathrm{ml}$ thrombin concentrations, and under CSPS, were tested in LAOStress experiments. The results, in terms of changes in linear elastic compliance and nonlinear compliances and the identified regions, were found to be very similar to fibrin clots with identical composition, and under SAOS. Increasing levels of applied unidirectional stress led to a narrower stress range in Region 4 which subsequently led to a decrease in eventual fracture stress of the clots. This could be because the level of unidirectional stress affects the formed clot's microstructure (i.e. higher level of unidirectional stress leads to higher $d_{f}$ and a more dense network) (Badiei et al. 2015). These results are important for understanding clots formed in vivo, which experience a unidirectional shear stress during their formation due to the flow of blood, and how these haemodynamic forces might influence the tendency of the clot to resist structural damage. The clots formed under conditions of higher shear stresses (such as those formed in patients with diseases such as atherosclerosis, stenosis and high blood pressure) have a lower fracture stress and might be more prone to embolism.

\subsection{Conclusion}

This chapter explored the LAOStress properties of mature fibrin clots. The effect of 
different clot microstructures - created by the addition of different thrombin concentrations or different levels of unidirectional stress in CSPS measurements was investigated. It was found that the nonlinear regions, and fracture stress were affected by the clot's microstructure. Fibrin clots are a useful model system for studying the nonlinear properties of blood clots, because the fibrin network provides the microstructure and mechanical properties for the clot to perform its haemostatic function. It also allows researchers to develop appropriate testing methods prior to studying human blood samples. Despite the benefits of this model system, it also has some insufficiencies, such as its inability to account for any cellular elements, which are abundant in a blood clot, and its lack of coagulation factors and enzymes that control the biochemistry of clot formation and the eventual clot lysis. Using the knowledge found here pertaining to the development of the LAOStress technique and the nonlinear properties of fibrin clots, the next chapter focuses on the LAOS characterisation of whole blood clots. 


\section{LAOS characterisation of whole blood}

\subsection{Aims and objectives}

This chapter explores the nonlinear viscoelastic properties of whole blood clots using both LAOStrain and LAOStress. Irreversible and reversible structural changes in whole blood clots were characterised using the LAOStress method developed and applied to fibrin clots in the previous chapter (Chapter 5.3.1). In order to explore the role of clot microstructure in the nonlinear viscoelastic properties of blood clots, clots with different values of $d_{f}$ were created by adding heparin (an anticoagulant) or thrombin (a procoagulant) to the sample before clotting was initiated. Whole blood clots were also created under controlled stress parallel superposition (CSPS), in order to mimic the conditions encountered due to haemodynamic forces in vivo. The relationship between the $d_{f}$ of the incipient blood clots and the nonlinear behaviour (in both LAOStrain and LAOStress) was investigated, with specific interest in the fracture stress of the mature clots. This serves to explore whether characterisation of the structure formed in coagulating whole blood, as measured by the rheometrically derived fractal dimension, provides a potential biomarker for the embolism (fracture) of the eventual mature clot.

\subsection{Materials}

\subsubsection{Whole blood clots}

Blood samples were collected from healthy volunteers following informed consent and under full ethical approval (REC Ref No. 13/WA/0190). Blood was withdrawn from an antecubital vein by venipuncture using a 21-gauge butterfly needle (Greiner Bio-One $\mathrm{GmbH}$, Austria), and the blood was collected into 9NC Vacuette ${ }^{\circledR}$ tubes (9 $\mathrm{ml}$, Greiner Bio-One GmbH, Austria), which contains anticoagulant, 3.8 \% sodium citrate to form a mixture of 9:1 V/V ratio of blood to citrate. Therefore, using citrated blood would allow performing more experiments form a single venepuncture. The first tube $(3 \mathrm{ml})$ was discarded, and the remaining tubes were used for the 
experiments. This ensures that a minimum amount of tissue factor was present in the blood used. Whole blood collected into the following tubes was used in the citrated blood experiments. Sodium citrate binds to the calcium ions in the blood, thus inhibiting several reactions within the coagulation cascade and preventing it from clotting. Prior to each experiment the clotting was initiated by adding Calcium Chloride, $\mathrm{CaCl}_{2}$ (Sigma-Aldrich, UK) to the blood to a final concentration of 0.005 M. The tubes containing citrated blood were used within 4 hours of collection, which is the recommended maximum storage period during which testing should be performed (Tripathi et al. 2017).

In experiments where the samples of blood were used immediately, Sodium Citrate was not required. Whole blood was collected into Vacuette ${ }^{\circledR}$ tubes (Greiner Bio-One $\mathrm{GmbH}$, Austria), which did not contain anticoagulant. The whole blood was then loaded on to the rheometer plate, immediately (within one minute) following blood collection. Clot structures were manipulated by adding a procoagulant or an anticoagulant, thrombin or heparin, to the blood sample, respectively. In order to reduce the $d_{f}$, heparin (Sigma-Aldrich, UK) was added to create a final concentration of $0.2 \mathrm{U} / \mathrm{ml}$ heparin in the sample. In order to increase the $d_{f}$, thrombin (Enzyme Research Laboratories, UK) was added to a final concentration of $0.025 \mathrm{NIH} / \mathrm{ml}$. These concentrations were selected as they had been previously shown to sufficiently change the value of $d_{f}$ (Evans et al. 2010).

\subsection{Methods}

\subsubsection{Gel Point measurements}

Gel Point measurements were conducted using an AR-G2 controlled stress rheometer. (TA Instruments, UK) fitted with parallel plate geometry $(60 \mathrm{~mm}$ diameter plates, and $400 \mu \mathrm{m}$ gap). All experiments were conducted at a constant temperature of $37^{\circ} \mathrm{C}$. The sample was subjected to SAOS stress waveforms at four different frequencies, ranging between 0.2 and $2 \mathrm{~Hz}$, and the resulting strain response was measured. Analysis of the waveforms provide viscoelastic parameters such as linear viscoelastic moduli, $G^{\prime}$ and $G^{\prime \prime}$. At the Gel Point $G^{\prime}$ and $G^{\prime \prime}$ show a power law 
dependence, $\alpha$, on the frequency of oscillation, such as $G^{\prime}(\omega) \sim G^{\prime \prime}(\omega) \sim \omega^{\alpha}$, whilst the phase angle, $\delta$, which is the lag between stress and strain becomes independent of frequency.

In the case of the LAOStrain experiments, some of the blood sample was transferred to the AR-G2 rheometer for Gel Point detection, whilst the remainder of the sample was transferred to the ARES-G2 rheometer for LAOStrain testing, and both experiments were started at the same time. The ARES-G2 rheometer was not used for Gel Point detection, because it lacks sensitivity at the low torque levels generated at the Gel Point, which is why the AR-G2 rheometer was used for the Gel Point detection. In the LAOStress experiments, the Gel Point of the sample was measured within the SAOS step of the procedure, in which the formation of the clot was also monitored.

\subsubsection{LAOStrain of whole blood clots}

LAOStrain measurements were conducted using an Ares-G2 controlled strain rheometer (TA Instruments, UK) fitted with a narrow gap double wall concentric cylinder (rotor outer diameter $43.92 \mathrm{~mm}$, inner diameter $40.76 \mathrm{~mm}$; stator inner diameter $40.00 \mathrm{~mm}$; cylinder immersed height $59.50 \mathrm{~mm}$ ) measuring system. The temperature was maintained at $37^{\circ} \mathrm{C}$ using a Peltier temperature controlling system. Prior to all experiments, samples were surrounded with a thin layer of low viscosity silicon oil in order to prevent evaporation. The clot formation was monitored in a "time sweep" measurement by applying SAOS strain waveform to the sample, $1 \%$ strain at $1 \mathrm{~Hz}$ frequency. The linear viscoelastic moduli were followed during clot formation, and the clot was considered fully formed once the storage modulus, $G^{\prime}$, reached a plateau value, this step typically took 10-15 times the gel time. Following that LAOStrain experiments were conducted on the mature blood clot, by applying a "strain sweep", in a stepwise mode with increasing levels of applied sinusoidal strain amplitudes to the clot, from 0.1 to $400 \%$. The data acquisition was set in transient mode in the Trios software. 5 seconds of conditioning time was applied prior to taking the data, in order to collect steady state data sets. 


\subsubsection{LAOStress of whole blood}

LAOStress measurements were conducted using an AR-G2 controlled stress rheometer (TA Instruments, UK) fitted with parallel plate geometry (60 mm diameter plates, and $400 \mu \mathrm{m}$ gap). The temperature was maintained at $37^{\circ} \mathrm{C}$. Blood clot formation was monitored in a SAOS experiment, in which $0.094 \mathrm{~Pa}$ stress was applied to the material, until it reached its maximum $G^{\prime}$ value. Following that, LAOStress tests were conducted on the fully formed blood clot, and the linear viscoelastic properties were probed by the application of SAOS (at a stress of $5 \mathrm{~Pa}$ ) between each of the incremental changes in stress amplitudes of the LAOStress test. Each stress amplitude was applied until the linear or nonlinear rheological response reached a steady state (the magnitude of the strain response did not change by more than $2 \%$ in SAOS and $0.2 \%$ in LAOStress between consecutive waveforms). The effects of different clot structures were studied by measuring whole blood, or heparinised whole blood $(0.2 \mathrm{U} / \mathrm{ml}$ heparin), or whole blood with thrombin $(0.025$ $\mathrm{NIH} / \mathrm{ml}$ thrombin). Experiments were repeated five times for each scenario (i.e. whole blood, whole blood with heparin, or whole blood with thrombin).

\subsubsection{SEM imaging of whole blood clots}

SEM images of fully formed whole blood clots were taken using scanning electron microscope (Hitachi 4800 S, Hitachi High-Technologies Pte Ltd., Singapore). The structure of some of the SEM imaged blood clots were modified by mixing 0.025 $\mathrm{NIH} / \mathrm{ml}$ thrombin or $0.2 \mathrm{U} / \mathrm{ml}$ heparin into the sample. All samples were transferred to a Petri dish and washed three times with $50 \mathrm{mM}$ Sodium Cacodylate-HCl Buffer solution ( $\mathrm{pH} 7.2-7.4$, SPI Supplies) in every 10 minutes in order to remove excess salt. The samples were fixed overnight in $2 \%$ Glutaraldehyde (Sigma Aldrich, UK), following that the gels were dehydrated by adding a series of graded concentrations (30\% to $100 \%$ ) of ethanol. The dehydrated samples were rinsed with $50 \%$ Hexamethyldisilazane solution (HMDS) (Sigma Aldrich, UK) in $100 \%$ ethanol for 10 minutes under a fume hood, and then rinsed three times with $100 \%$ HMDS and left to dry overnight. All samples were mounted, sputter-coated with a thin layer of gold-palladium (approximately $15 \mathrm{~nm}$ ) using an SEM coating unit E5100 (Polaron Instruments Inc., West Sussex, England) at $2.2 \mathrm{kV}$ and $20 \mathrm{~mA}$ for $1.5 \mathrm{~min}$, and 
examined in a Hitachi $4800 \mathrm{~S}$ scanning electron microscope (Hitachi $4800 \mathrm{~S}$, Hitachi High-Technologies Pte Ltd., Singapore). Duplicates of each clot were made, and several fields on each clot were examined before choosing fields that were characteristics of the entire clot.

\subsubsection{LAOSress of whole blood clots formed under CSPS}

The effects of an imposed unidirectional shear field during clot formation on the LAOS properties of whole blood clots were studied. CSPS utilising a unidirectional stress, $\sigma_{s}=0.047 \mathrm{~Pa}$, was applied and the sample was subjected to four different frequencies between 0.2 and $2 \mathrm{~Hz}$ in order to detect the Gel Point. Once the material has obtained a Gel Point, the unidirectional flow shear rate, $\dot{\gamma}_{s}$, becomes negligible, which suggests that the value of $G_{\|}^{\prime}, G_{\|}^{\prime \prime}$ and $\delta_{\|}$are approximately equal to their corresponding measures in SAOS (Badiei 2015). The values of $d_{f}$ of the incipient clots formed under CSPS were calculated using the frequency independent value of $\delta_{\|}$. LAOStress studies of the mature clots formed under CSPS were then studied using the procedure outlined in Section 6.3.3.

\subsection{Results}

\subsubsection{LAOStrain of whole blood clots}

Citrated blood samples were recalcified and tested in Gel Point and in LAOStrain experiments. Although all samples were tested within the recommended 4 hour period, both the values of fracture stress and fracture strain were affected by the length of the time between blood collection and rheological testing. The results in Table 6.1 show that delayed LAOStrain testing of the samples resulted in decreased values of fracture stress and fracture strain. In order to avoid this problem, noncitrated whole blood samples were collected and tested on the rheometer immediately after taking the blood. 
Table 6.1 Values of fracture stress and fracture strain measured for citrated blood samples tested immediately after blood collection and one hour after blood collection.

\begin{tabular}{||c|c|c|c|c||}
\hline \multirow{3}{*}{} & \multicolumn{2}{|c|}{$\begin{array}{r}\text { Testing immediately after } \\
\text { blood collection }\end{array}$} & \multicolumn{2}{c|}{$\begin{array}{r}\text { Testing 1 hour after blood } \\
\text { collection }\end{array}$} \\
\cline { 2 - 5 } & $\begin{array}{c}\text { Fracture } \\
\text { stress }\end{array}$ & $\begin{array}{c}\text { Fracture } \\
\text { strain }\end{array}$ & $\begin{array}{c}\text { Fracture } \\
\text { stress }\end{array}$ & $\begin{array}{c}\text { Fracture } \\
\text { strain }\end{array}$ \\
\cline { 2 - 5 } & Pa & \% & Pa & $\%$ \\
\hline Sample 1 & - & - & 94.82 & 39.42 \\
\hline Sample 2 & 155.79 & 98.94 & 74.36 & 39.30 \\
\hline Sample 3 & 160.44 & 98.93 & 83.50 & 38.75 \\
\hline
\end{tabular}

Fracture stress values for whole blood clots were measured in "strain sweep" LAOStrain measurements. Different clot structures were created by the addition of heparin to achieve a relatively lower $d_{f}$ or by the addition of thrombin to achieve a relatively higher $d_{f}$. Results show that the fracture stress of the fully formed clots decreased with increasing values of $d_{f}$ (Figure 6.1). The lowest value of fracture stress of $40 \mathrm{~Pa}$ was measured in a clot with $d_{f}$ of 1.80 , whilst the highest value of fracture stress $(206 \mathrm{~Pa})$ was measured in a clot formed with $d_{f}$ of 1.54 . By fitting a linear trend line on the dataset, extrapolation of the fitting predicts $d_{f}$ value of 1.90 for a fracture stress approaching the zero limit.

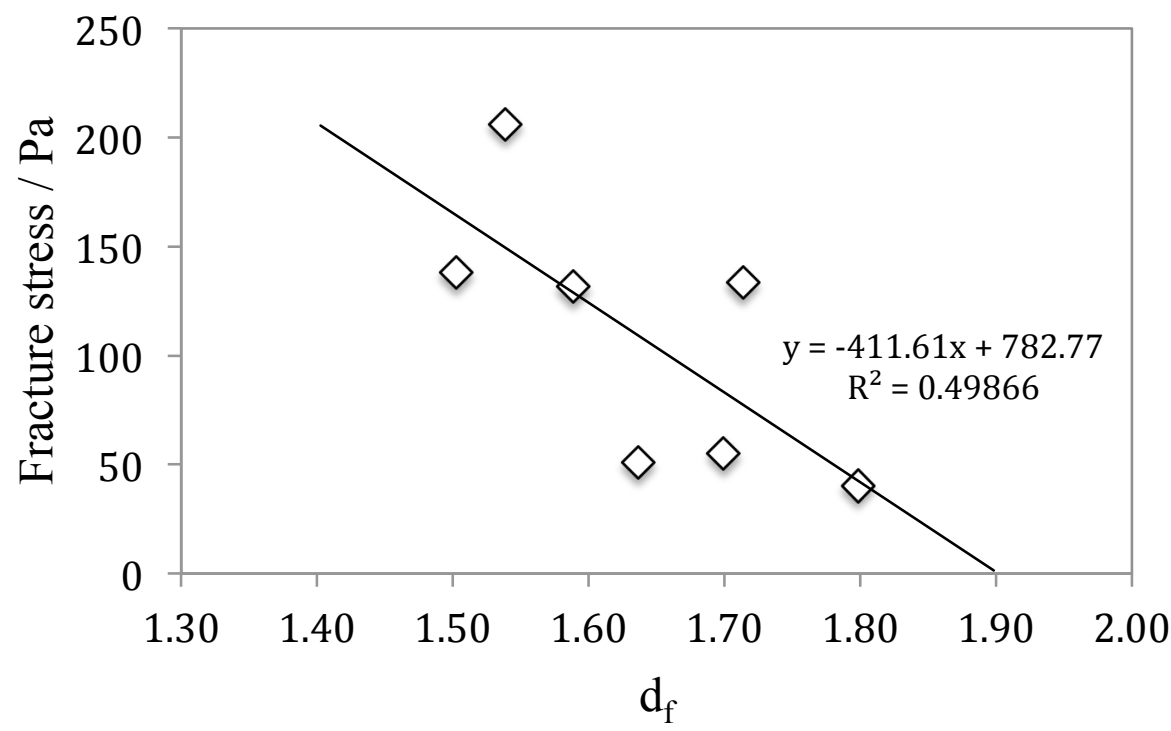

Figure 6.1 Fracture stress of whole blood clots measured as a function of fractal dimension in LAOStrain measurements. 


\subsubsection{LAOStress of whole blood clots: Lissajous-Bowditch curves}

Figure 6.2 displays Lissajous-Bowditch curves of whole blood clots constructed from the original and smoothed stress and strain datasets. Ringing in the stress and strain waveforms was observed at higher applied stress levels similar to that seen in fibrin clots. In order to extract reliable nonlinear compliance values from the data, the stress and strain waveforms were smoothed by applying the same method described in Chapter 5.4.1.1.

At relatively lower levels of stresses (Figure 6.2a and 6.2b), the original and smoothed Lissajous-Bowditch curves appear identical. Ringing appears in the original waveforms at higher levels of stresses, and resulting in secondary loops in the Lissajous-Bowditch curve, as shown in Figure 6.2c. Secondary loops were eliminated by applying a smoothing spline curve fitting which permitted the calculation of $J_{M}^{\prime}$.
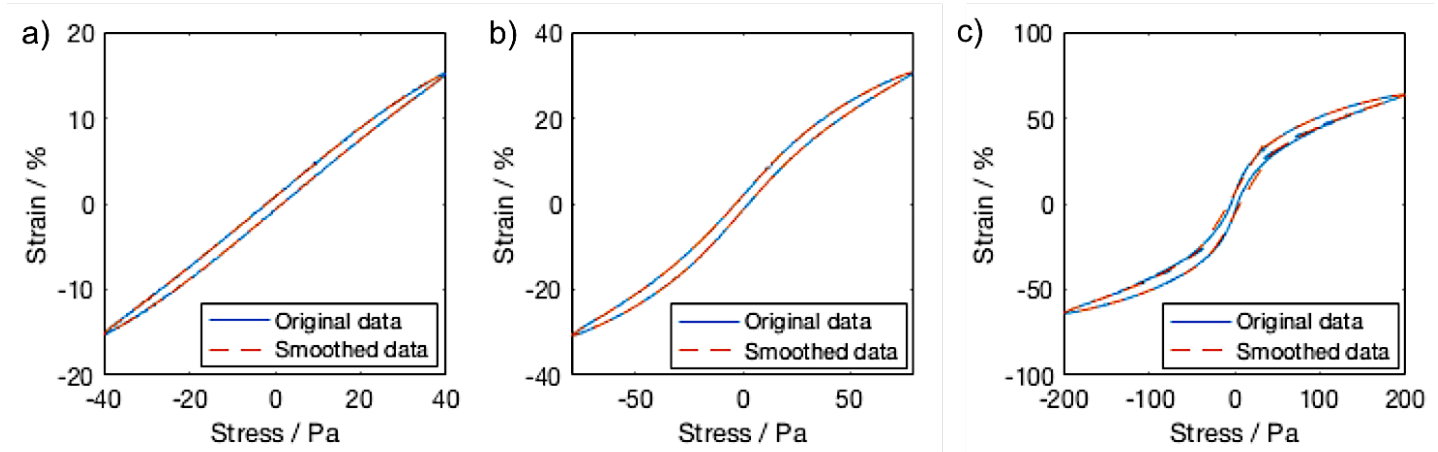

Figure 6.2 Comparison of Lissajous-Bowditch curves of whole blood clots using original (blue solid line) and smoothed (red dashed line) datasets for input stress levels of (a) $40 \mathrm{~Pa}$, (b) $80 \mathrm{~Pa}$, and (c) $200 \mathrm{~Pa}$.

\subsubsection{LAOStress of whole blood clots: probing structural changes}

Different regions of nonlinear viscoelastic behaviour were seen in the experiments involving alternating application of SAOS and LAOStress, similarly to the nonlinear regions observed for mature fibrin clots in the previous chapter (Figure 6.3). However, in the case of whole blood clots the regions appear at considerably smaller stress values, and the characteristics of each region in term of the changes in linear and nonlinear compliances differ to what was observed in fibrin clots. Both linear 
and nonlinear compliances increased with increasing levels of applied stress, suggesting strain stiffening behaviour of the blood clots. In the linear viscoelastic region (LVR) $\left(\sigma_{0}<40 \mathrm{~Pa}\right)$, the values of nonlinear compliances, $J_{M}^{\prime}$ and $J_{L}^{\prime}$, are equal, and the value of linear compliance following the applied, increasing level of stress remains unchanged. In nonlinear Region 1 , the values of $J_{M}^{\prime}$ and $J_{L}^{\prime}$ are not equal (50 $\mathrm{Pa}<\sigma_{0}<100 \mathrm{~Pa}$ ), indicating that the material's response is nonlinear. $J_{M}^{\prime}$ increases whilst $J_{L}^{\prime}$ is constant, and the linear compliance slightly increases. In Region 2 , a steeper increase in both $J_{M}^{\prime}$ and linear compliance, and slight decrease in $J_{L}^{\prime}$ was observed $\left(150 \mathrm{~Pa}<\sigma_{0}<300 \mathrm{~Pa}\right)$. In the last region the complete fracture of the clot occurs as indicated by the rapid increase in the strain leading the rheometer plate to overspeed $\left(300 \mathrm{~Pa}<\sigma_{0}\right)$.

In the case of the heparinised whole blood clots (discussed later in Chapter 6.4.4), four different nonlinear regions were identified (Table 6.2). Characteristics of Region 3 cannot be observed, hence Region 3 is absent in the case of the clot shown in Figure 6.3. For the purpose of consistency, Region 4 is used to describe the final region that leads to the complete fracture of the clot.

Table 6.2 Summary of the different characteristics of each nonlinear region in terms of linear and nonlinear compliances. $\uparrow$ represents increase, $\downarrow$ represents decrease, whilst $=$ represents unchanging values.

\begin{tabular}{|c|c|c|c|c|}
\hline & Region 1 & Region 2 & Region 3 & Region 4 \\
\hline$J^{\prime}$ & $\uparrow$ & $\uparrow$ & $\uparrow$ & $\uparrow$ \\
\hline$J_{M}^{\prime}$ & $\uparrow$ & $\uparrow$ & $=$ & $\uparrow$ \\
\hline$J_{L}^{\prime}$ & $\uparrow$ or $=$ & $\downarrow$ & $\downarrow$ & $\uparrow$ \\
\hline
\end{tabular}



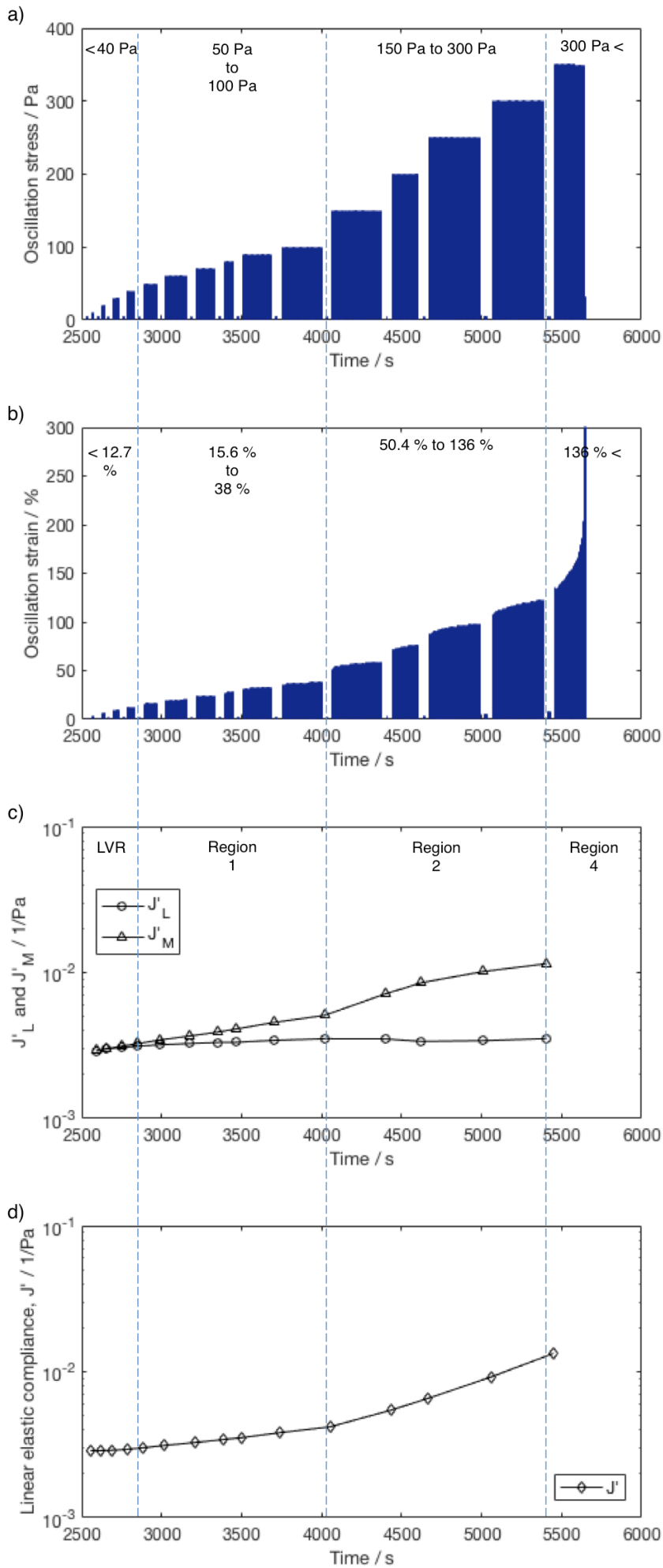

Figure 6.3 Result of alternating SAOS and LAOStress measurements on a whole blood clot formed with fractal dimension of 1.61; (a) the applied stress versus time profile, (b) the resulting strain response versus time profile, (c) large-stress and minimum-stress elastic compliances (as measured by LAOStress), and (d) linear elastic compliance (as measured by SAOS). The results reveal several nonlinear viscoelastic regions (numbered 1-4, as illustrated by the dashed lines). 


\subsubsection{LAOStress of whole blood clots: Effects of clot microstructure}

The microstructure of whole blood clots was modified by the addition of 0.025 $\mathrm{NIH} / \mathrm{ml}$ thrombin or $0.2 \mathrm{U} / \mathrm{ml}$ heparin, and the nonlinear viscoelastic behaviour of these clots was investigated using LAOStress. Figure 6.4 shows that in the case of whole blood mixed with thrombin $\left(d_{f}=1.72\right)$, only two regions can be distinguished prior to the final region, whereby the clot fully fractures $\left(150 \mathrm{~Pa}<\sigma_{0}\right)$. The limiting stress value of the LVR in the presence of thrombin is lower $\left(\sigma_{0}<20 \mathrm{~Pa}\right)$ compared to what was measured for the whole blood clot $\left(\sigma_{0}<40 \mathrm{~Pa}\right)$. In Region 1 , the linear elastic compliance and $J_{M}^{\prime}$ gradually increase, and $J_{L}^{\prime}$ remains level with increasing levels of stress $\left(30 \mathrm{~Pa}<\sigma_{0}<100 \mathrm{~Pa}\right)$.

Figure 6.5 shows the LAOStress behaviour of a whole blood clot that has been modified by the addition of heparin $\left(d_{f}=1.45\right)$. The results are different from what can be seen in whole blood or whole blood and thrombin. In the nonlinear viscoelastic range, four different regions can be identified based on the observed changes in measured linear and nonlinear compliances. The limiting stress value of the LVR is similar to that of the whole blood clots $\left(\sigma_{0}<40 \mathrm{~Pa}\right)$. In Region 1 (50 Pa $<$ $\left.\sigma_{0}<100 \mathrm{~Pa}\right), J_{L}^{\prime}$ slightly increases, while both $J_{M}^{\prime}$ and linear elastic compliance gradually increase with increasing levels of stress. Region $2\left(150 \mathrm{~Pa}<\sigma_{0}<250 \mathrm{~Pa}\right)$ is similar to that of the whole blood sample (Figure 6.3), insofar that there is an increase in both $J_{M}^{\prime}$ and linear elastic compliance, and a decrease in $J_{L}^{\prime}$. An additional region not seen in whole blood clots with or without thrombin, Region 3 (300 $\mathrm{Pa}<$ $\sigma_{0}<550 \mathrm{~Pa}$ ), can be observed, in which $J_{M}^{\prime}$ levels off, whilst further increases in linear elastic compliance and decrease in $J_{L}^{\prime}$ in response to increases in stress can be seen. Complete clot fracture occurs in Region $4\left(600 \mathrm{~Pa}<\sigma_{0}\right)$, as indicated by the significant increase in oscillation strain. 

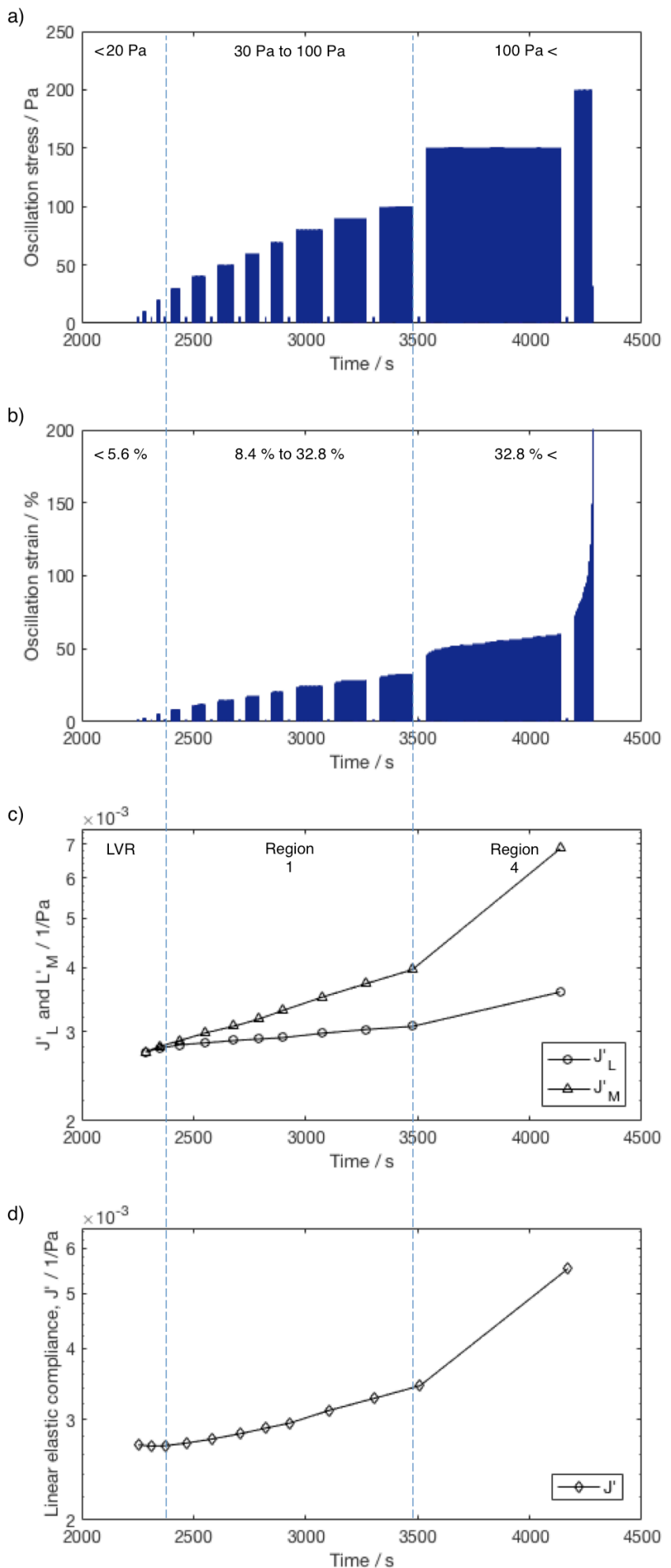

Figure 6.4 Result of alternating SAOS and LAOStress measurements on a whole blood clot formed with the addition of $0.025 \mathrm{NIH} / \mathrm{ml}$ thrombin $\left(d_{f}=1.72\right)$; (a) the applied stress versus time profile, (b) the resulting strain response versus time profile, (c) large-stress and minimum-stress elastic compliances (as measured by LAOStress), and (d) linear elastic compliance (as measured by SAOS). The results reveal several nonlinear viscoelastic regions (numbered 1-4, as illustrated by the dashed lines). 


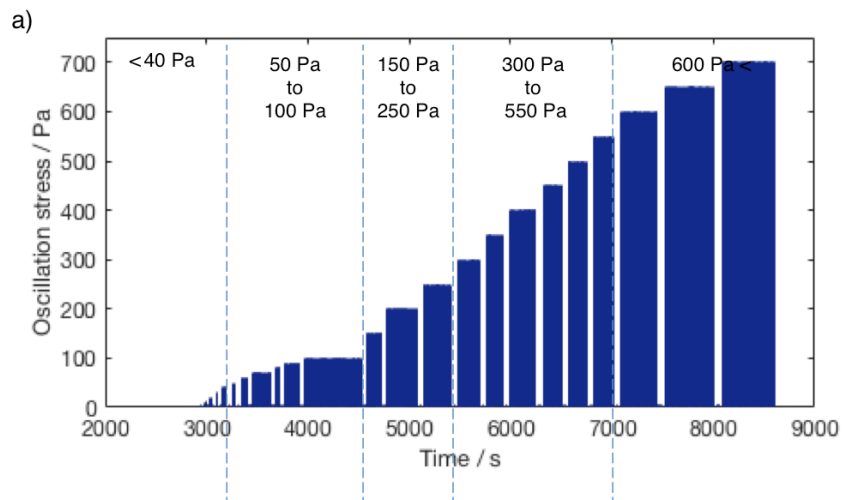

b)

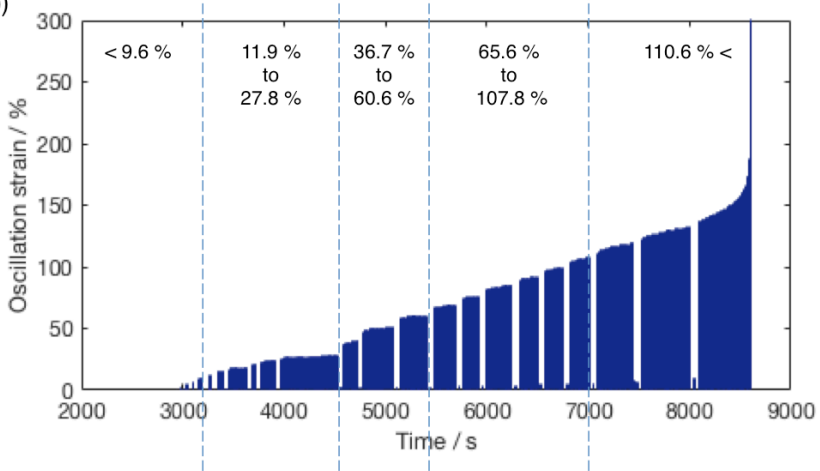

c)
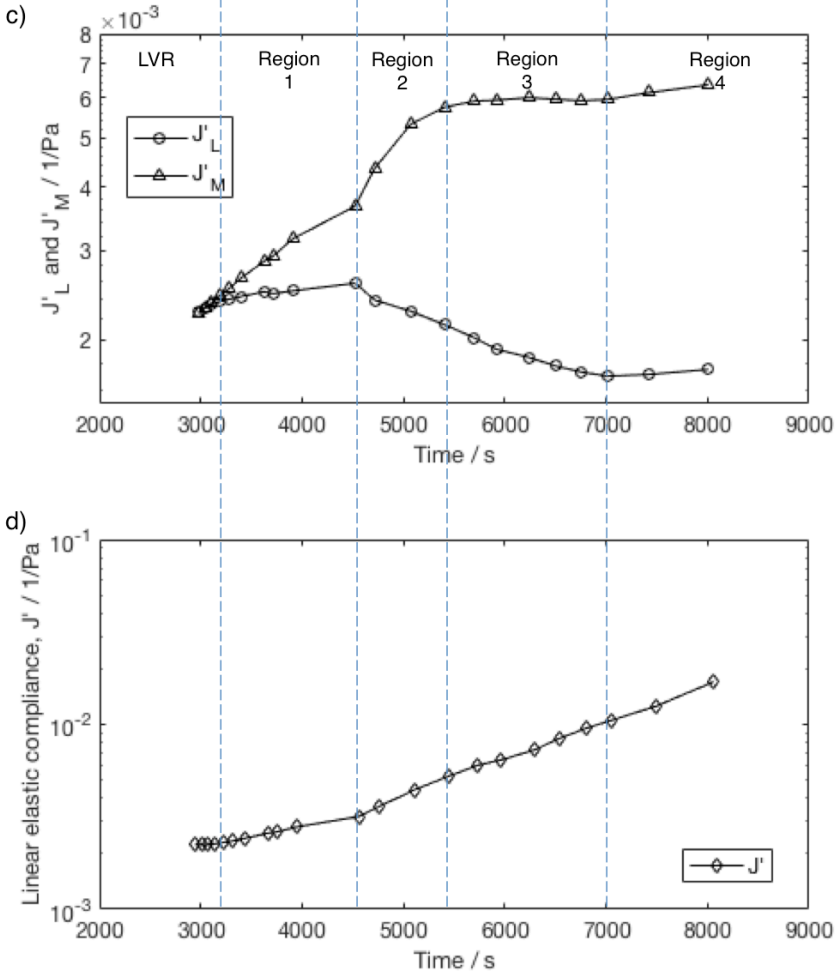

Figure 6.5 Result of alternating SAOS and LAOStress measurements on a whole blood clot formed with the addition of $0.2 \mathrm{U} / \mathrm{ml}$ heparin $\left(d_{f}=1.45\right)$; (a) the applied stress versus time profile, (b) the resulting strain response versus time profile, (c) large-stress and minimum-stress elastic compliances (as measured by LAOStress), and (d) linear elastic compliance (as measured by SAOS). The results reveal several nonlinear viscoelastic regions (numbered 1-4, as illustrated by the dashed lines). 
Figure 6.6 shows the observed linear and nonlinear viscoelastic regions in terms of the applied oscillatory stress ranges. Experiments were repeated five times for each system, and the displayed error bars represent standard deviations. It can be seen that the regions were influenced by the clot's microstructure (i.e. $d_{f}$ values of the incipient clot), which was modified by the addition of thrombin (i.e. more dense network, relatively high $d_{f}$ ) or heparin (i.e. more open network, relatively low $d_{f}$ ). Three different nonlinear regions were observed for whole blood clots $\left(d_{f}=1.66 \pm 0.037\right)$. In the case of the more open clot structures (i.e. formed with the addition of heparin leading to a relatively lower $d_{f}=1.48 \pm 0.024$ ), four different nonlinear regions were identified, and these regions shifted to higher levels of stress. Whilst, in the case of the more dense clots (i.e. formed with the addition of thrombin leading to a relatively high $\left.d_{f}=1.76 \pm 0.032\right)$ the regions shifted to lower levels of stress, and even led to the disappearance of some of the regions (i.e. Region 2 and Region 3 ).

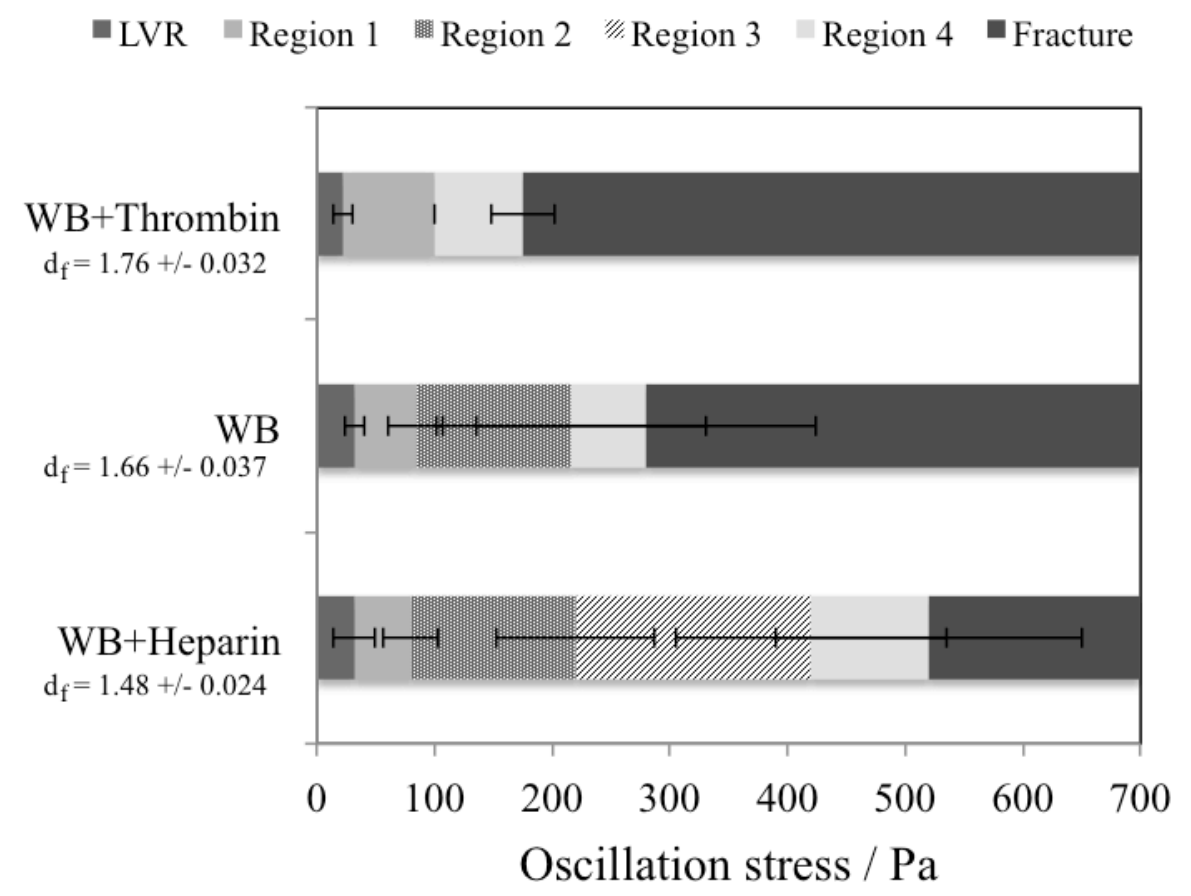

Figure 6.6 The effect of fractal dimension on the nonlinear viscoelastic regions measured for whole blood clots in terms on oscillatory stress

Figure 6.7 represents the observed linear and nonlinear viscoelastic regions in terms of oscillatory strain ranges. Similar to the oscillatory stress ranges, the strain ranges have shifted as a result of different clot structures (i.e. different $d_{f}$ values). Three 
different nonlinear regions were observed for whole blood clots $\left(d_{f}=1.66 \pm 0.037\right)$. In the case of the addition of heparin $\left(d_{f}=1.48 \pm 0.024\right)$ the regions shifted to higher levels of strain, and an additional region can be identified. Whilst, in the case of the addition of thrombin $\left(d_{f}=1.76 \pm 0.032\right)$ the regions shifted to lower levels of strain, which lead to the disappearance of Region 2 and Region 3.

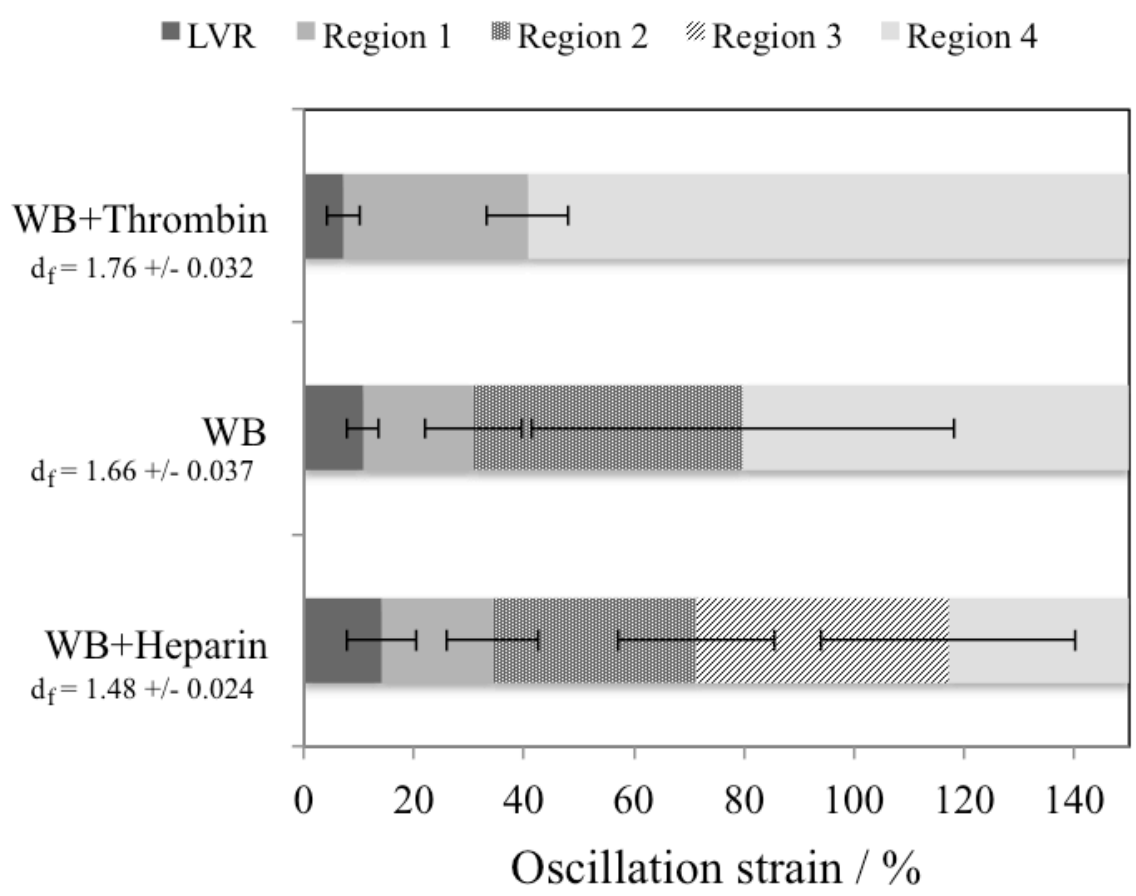

Figure 6.7 The effect of fractal dimension on the nonlinear viscoelastic regions measured for whole blood clots in terms of oscillatory strain values

\subsubsection{SEM imaging of whole blood clots}

The structure of the unadulterated blood clots and clots formed with the addition of heparin or thrombin was visualised using scanning electron microscopy. Figure 6.8 shows the SEM images of the different blood clots. Compared to the unadulterated blood clots, those formed with the addition of thrombin show a more dense fibrin network, whilst conversely, clots formed with the addition of heparin display a more open structure. 

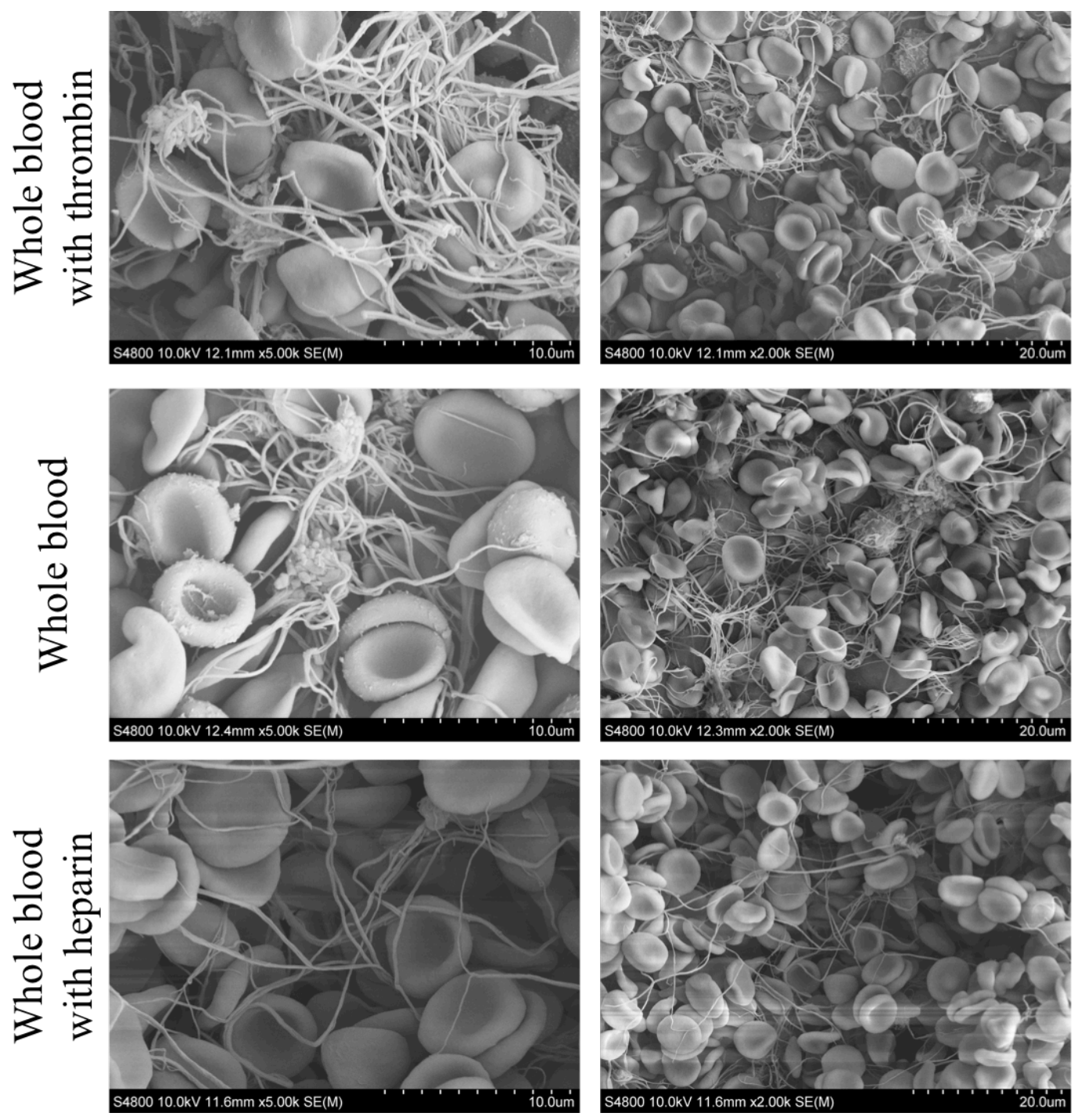

Figure 6.8 SEM images of whole blood clots, and whole blood clots in the presence of $0.025 \mathrm{NIH} / \mathrm{ml}$ thrombin or $0.2 \mathrm{U} / \mathrm{ml}$ heparin

\subsubsection{Whole blood clots formed under CSPS}

LAOStress results of whole blood clots formed under CSPS showed similar behaviour to blood clots formed in the presence of thrombin. Figure 6.9 shows that three different regions can be distinguished at a high level of $d_{f}$ value $\left(d_{f}=2.05\right)$. The LVR, similar to the SAOS whole blood samples, occurs below $20 \mathrm{~Pa}$, whereby $J_{L}^{\prime}$ and $J_{M}^{\prime}$ are equal. In Region $1\left(30 \mathrm{~Pa}<\sigma_{0}<40 \mathrm{~Pa}\right) J_{L}^{\prime}$ is constant, whilst $J_{M}^{\prime}$ and the linear elastic compliance increase following increases in applied stress. While, the gradual increase in strain at stress levels above $50 \mathrm{~Pa}$ leads to full clot fracture. 


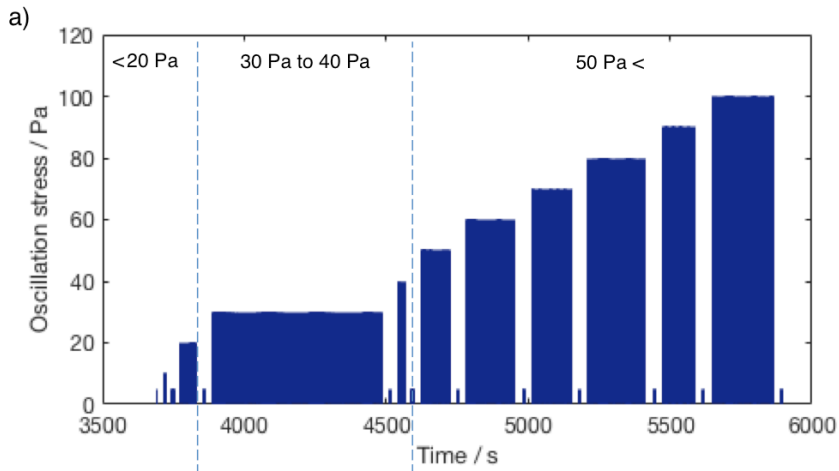

b)

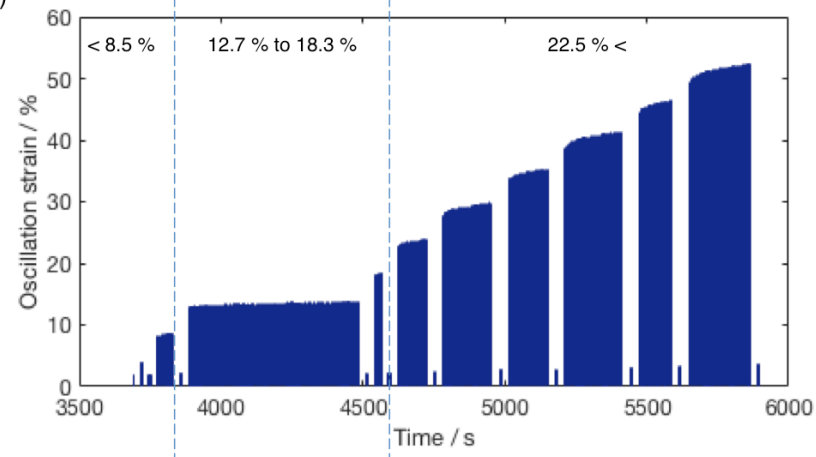

c)

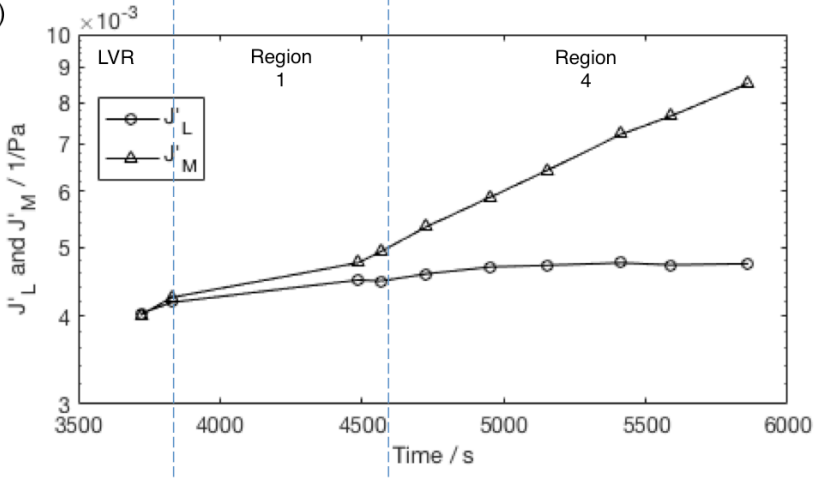

d)

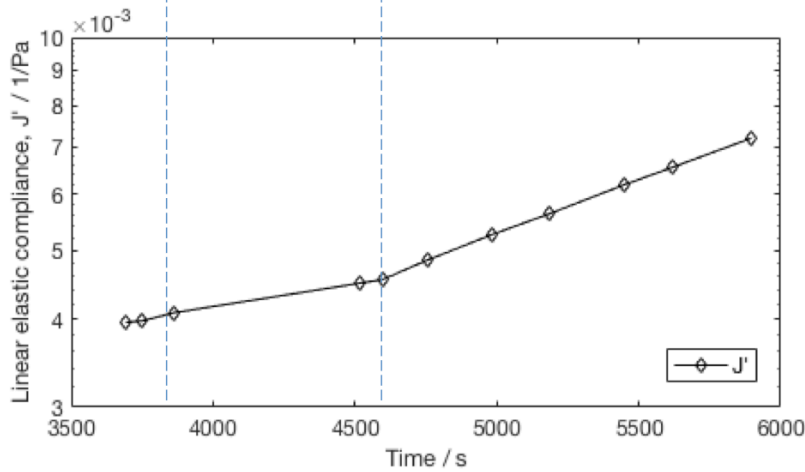

Figure 6.9 Result of alternating SAOS and LAOStress measurements on a whole blood clot formed under the application of CSPS with a higher fractal dimension value $\left(d_{f}=2.05\right)$; (a) the applied stress versus time profile, (b) the resulting strain response versus time profile, (c) large-stress and minimum-stress elastic compliances (as measured by LAOStress), and (d) linear elastic compliance (as measured by SAOS). The results reveal several nonlinear viscoelastic regions (numbered 1-4, as illustrated by the dashed lines). 
Similar to blood clots formed under SAOS, in the case of a blood clot formed under unidirectional stress with a lower $d_{f}$ value $\left(d_{f}=1.85\right)$, four different nonlinear regions can be distinguished by investigating the changes in linear and nonlinear compliances (Figure 6.10). The characteristics of each regions found in CSPS clots are similar to those found in SAOS whole blood clots. In the LVR $\left(\sigma_{0}<50 \mathrm{~Pa}\right)$, the linear elastic compliance is constant, and $J_{L}^{\prime}$ and $J_{M}^{\prime}$ are equal. This region is followed by the first nonlinear region, Region $1\left(60 \mathrm{~Pa}<\sigma_{0}<100 \mathrm{~Pa}\right)$, in which values of the nonlinear compliances change with increasing stress, $J_{L}^{\prime}$ slightly decreases, while both $J_{M}^{\prime}$ and linear elastic compliance gradually increase. In Region $2\left(150 \mathrm{~Pa}<\sigma_{0}<\right.$ $350 \mathrm{~Pa})$ both $J_{M}^{\prime}$ and linear elastic compliance further increase, whilst there is a decrease in $J_{L}^{\prime}$ following increases in applied stress. Region $3\left(400 \mathrm{~Pa}<\sigma_{0}<600 \mathrm{~Pa}\right)$ can be identified, as $J_{M}^{\prime}$ and $J_{L}^{\prime}$ are both constant, whilst a further increase in linear elastic compliance can be seen. Complete clot fracture occurs in Region 4 (600 $\mathrm{Pa}<$ $\sigma_{0}$ ), as indicated by the significant increase in oscillation strain. 

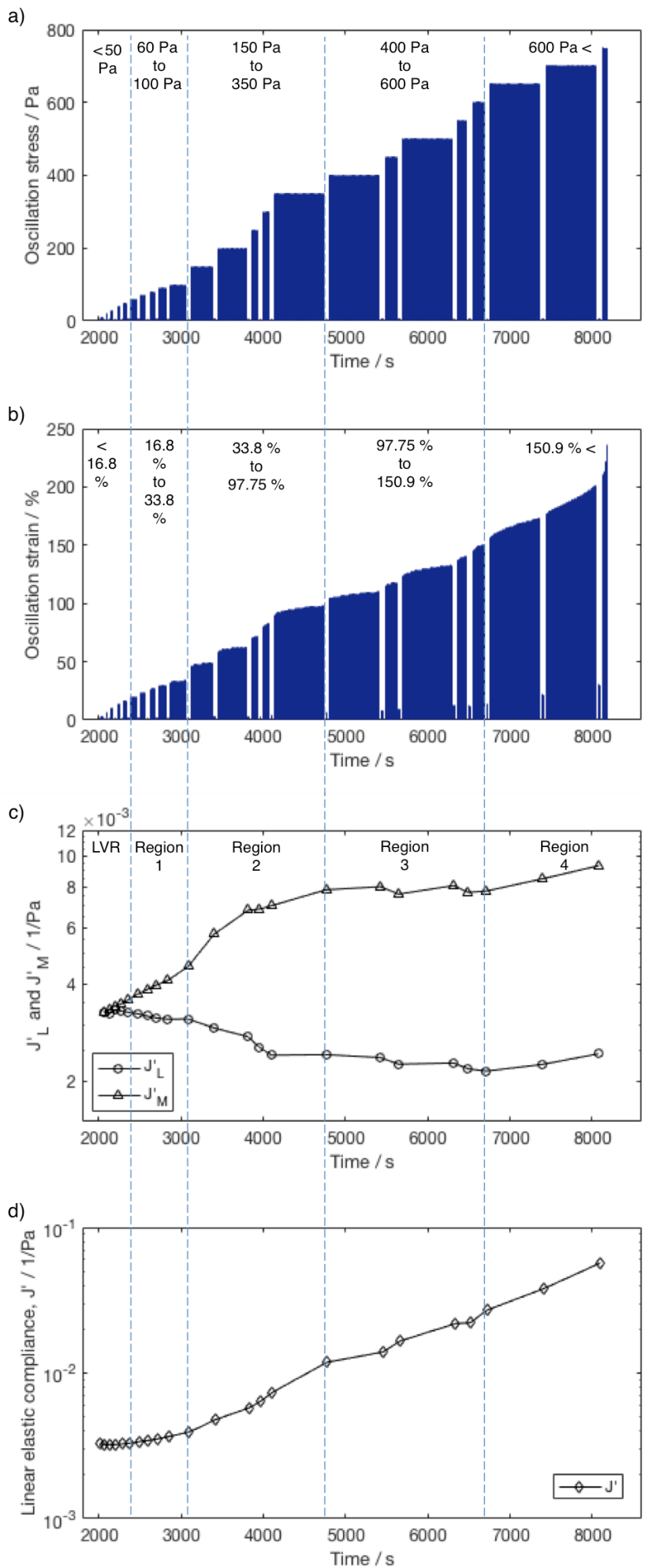

Figure 6.10 Result of alternating SAOS and LAOStress measurements on a whole blood clot formed under the application of CSPS with a lower fractal dimension value $\left(d_{f}=1.85\right)$; (a) the applied stress versus time profile, (b) the resulting strain response versus time profile, (c) large-stress and minimum-stress elastic compliances (as measured by LAOStress), and (d) linear elastic compliance (as measured by SAOS). The results reveal several nonlinear viscoelastic regions (numbered 1-4, as illustrated by the dashed lines). 
Different nonlinear regions were identified for blood clots formed under CSPS, and plotted as a function of measured incipient clot's $d_{f}$ in Figure 6.11. Similarly to the clots formed under SAOS, four different nonlinear regions are observed at lower values of $d_{f}$, and above a certain $d_{f}$ value (at 2.05), only two nonlinear regions are evident. The increase in the value of $d_{f}$ also caused a shift in the regions to lower oscillatory stress levels.

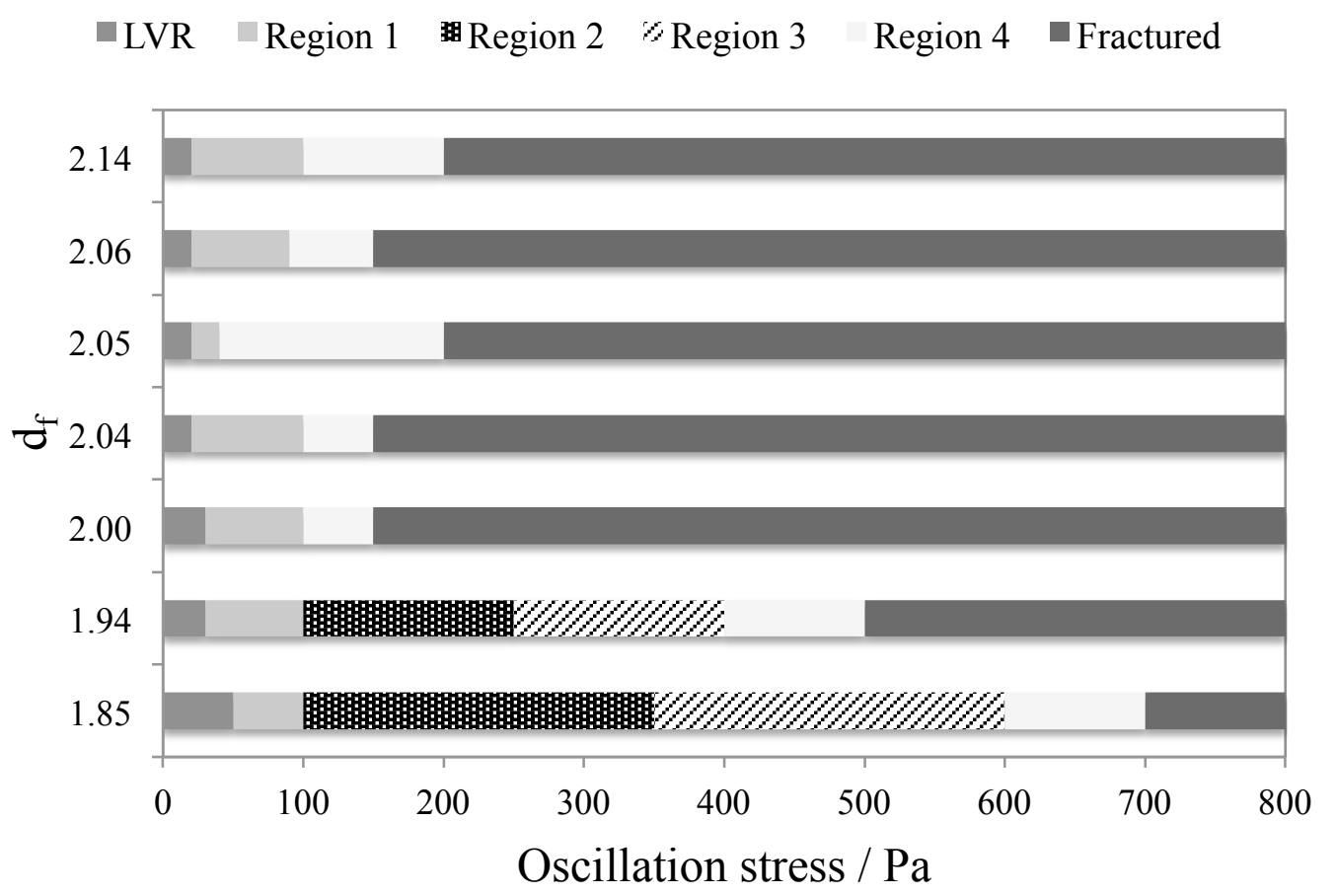

Figure 6.11 Nonlinear viscoelastic ranges in oscillatory stress levels as a function of discrete values of fractal dimension

Upon investigating the nonlinear regions in terms of oscillatory strain ranges, the results show similarities to those when displayed in terms of oscillatory stress ranges (Figure 6.12). As the value of $d_{f}$ is increasing, the strain ranges shift to lower levels of strains; and Region 2 and 3 cannot be observed above $d_{f}$ of 2.05 . 


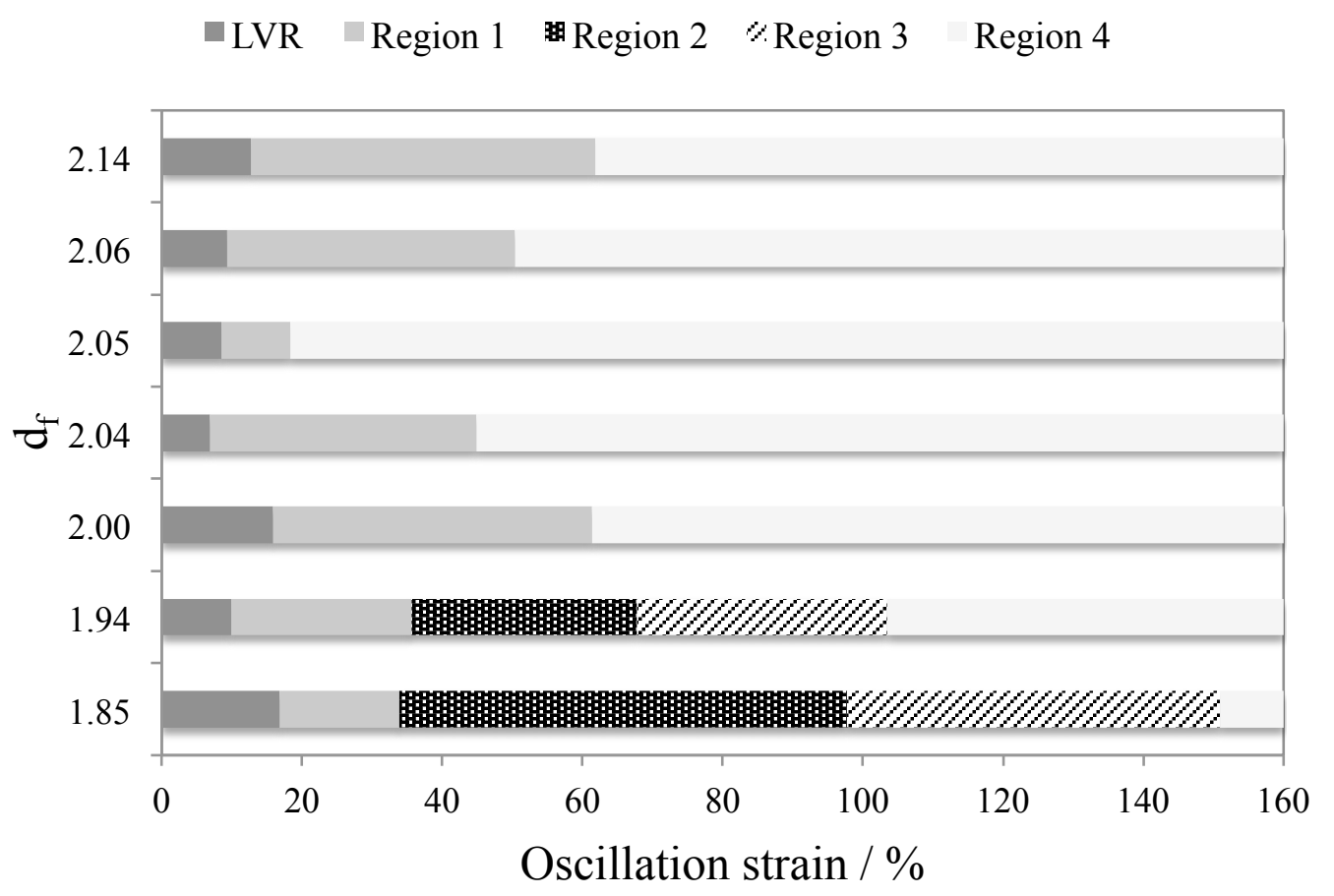

Figure 6.12 Nonlinear viscoelastic ranges in oscillatory strain levels as a function of discrete values of fractal dimension

\subsubsection{Relationship between fractal dimension and the fracture stress}

The results in Figure 6.13 show that fracture stress of the fully formed clot is influenced by the $d_{f}$ of the incipient clot. The microstructure of the whole blood clots were altered by adding thrombin or heparin in to the sample, or by forming clots under CSPS. As the value of $d_{f}$ increases, the fracture stress decreases. Whole blood clots formed under CSPS resulted in an increase in values of $d_{f}$, as demonstrated by a shift in the linear fit, and relationship between the fracture stress and $d_{f}$ was similar to clots formed under SAOS insofar that the fracture stress decreases with increasing values of $d_{f}$. 


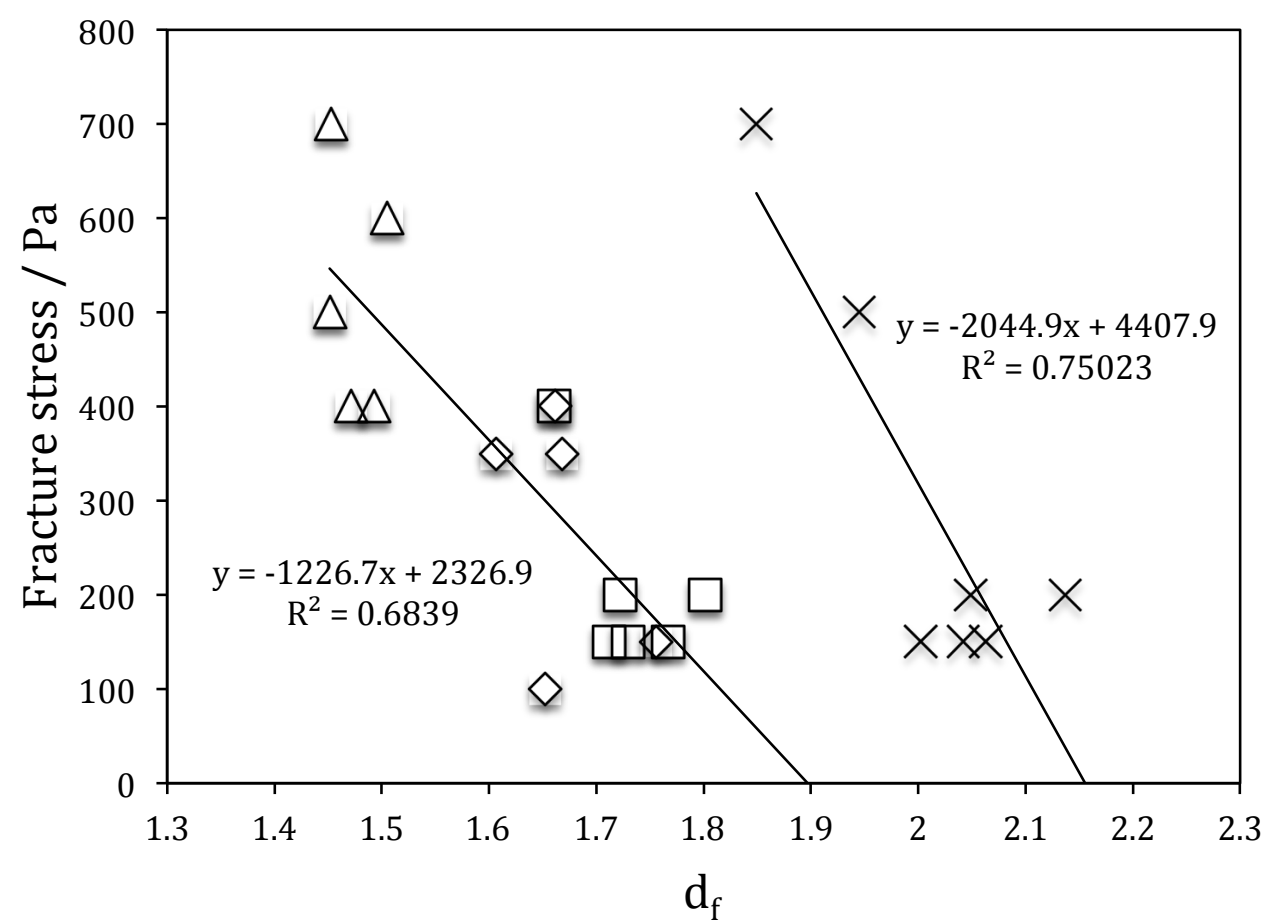

$\diamond$ Whole blood $\quad \square \mathrm{WB}+$ Thrombin $\quad \triangle \mathrm{WB}+$ Heparin $\quad \times \mathrm{WB}+\mathrm{CSPS}$

Figure 6.13 Measured fracture stress values of whole blood clots as a function of fractal dimension

By investigating the correlation between $d_{f}$ and fracture stress in the case of clots formed under SAOS, it can be seen in Figure 6.13 that extrapolation of the data predicts a $d_{f}$ of 1.9 for the zero limit fracture stress. Forming blood clots under CSPS shifted the $d_{f}$ values into a higher region, from 1.85 to 2.14 . Hence, extrapolation of the data suggests $d_{f}$ of 2.15 for the zero limit fracture stress for clots formed under CSPS.

\subsection{Discussion}

Preliminary LAOStrain study characterised the fracture properties of citrated blood, which was recalcified following different time periods prior to rheological testing, to initiate clotting. This study revealed that the length of time between blood collection and testing affects the nonlinear rheological response of the blood clots. Hence, whole blood was collected from healthy volunteers, immediately transferred to the rheometer plates, and allowed to coagulate whilst being probed by SAOS. Following attainment of the fully formed clot, as indicated by a plateau in $G^{\prime}$, the sample was 
subjected to increasing levels of strains in "strain sweep" experiments. The results showed that the value of $d_{f}$ of incipient clots correlates with the eventual fracture stress of the mature clot, with increasing values of $d_{f}$ corresponding to a reduced fracture stress. This finding is in agreement with the results in LAOStress characterisation of fibrin clots formed with different thrombin concentrations (see Chapter 5.4.5). The $d_{f}$ of incipient clots determines the microstructure of the fully formed clot, because the incipient clot microstructure is the template for ensuing clot growth (Curtis et al. 2013). Consequently, clots formed with a lower $d_{f}$ will form a more open, porous fibrin fibre network, whilst clots formed with a higher $d_{f}$ will result in a more dense and less porous network. Therefore, in the case of a lower $d_{f}$ value there is more available space for structural rearrangements as a consequence of deformation, which is a potential reason for the observed increase in fracture stress at lower $d_{f}$. In addition, it was found that, from extrapolation of the data, a $d_{f}$ of 1.9 corresponds to zero fracture stress. This suggests that a $d_{f}$ of 1.9 is an upper, limiting value for $d_{f}$, since a clot cannot exist with zero fracture stress and would not be physically meaningful.

Alternating LAOStress and Small Amplitude Oscillatory Shear was applied to fully formed whole blood clots to provide detailed information of reversible and irreversible structural changes, and to further investigate the likelihood of structural rearrangements of the fibrin network in the blood clots. Whole blood clots behaved similar to the fibrin clots (see Chapter 5.4.2), insofar that several distinct regions of nonlinear viscoelastic behaviour were seen up to the point of fracture. The relationship between the underlying microstructure of the fibrin network within the whole blood clots formed at the Gel Point, as characterised by $d_{f}$, with the nonlinear viscoelastic properties of the eventual (i.e. fully formed) network was investigated.

Previous work involving rheometrical detection of incipient clots formed in the presence of unfractionated heparin, a known anticoagulant that inhibits thrombin production, produce clots with a lower value of $d_{f}$ (Evans et al. 2010). Therefore, in the present study, the microstructure of blood clots was manipulated by the addition of heparin, in order to reduce the $d_{f}$, or thrombin, in an attempt to create more dense network structure, with higher $d_{f}$. In these experiments, a range of $d_{f}$ of the incipient clots were measured $\left(1.45<d_{f}<1.80\right)$ which resulted in different nonlinear 
viscoelastic behaviour of the mature clots. This might be expected since the incipient clot structure is known to be a template for ensuing clot growth (Curtis et al. 2013). Different nonlinear regions were observed for blood clots. However, the characteristics of these regions found in blood clots are markedly different to those found in fibrin clots, which might be expected, due to the presence of the cellular elements trapped within the fibrin network of the blood clot. The presence of cellular elements during fibrin polymerisation will affect the forming fibrin network, thus affecting the nonlinear properties of the clot (Weisel \& Litvinov 2013b). In particular, platelets will bind to the fibrin network and produce an increasing contractile stress as the network develops. Furthermore, as the fibrin network is deformed, the entrapped cells are likely to contribute to the bulk viscoelastic response by undergoing structural deformations themselves, moving around the pores within the network or even adhering to the fibrin network. The nonlinear regions observed in the LAOStress study of blood clots occur at lower levels of stresses compared to fibrin clots. This might be due to cellular elements: platelets, leucocytes, and mainly red blood cells are present in the system apart from the fibrin fibre network, that provides strength to the clot (Weisel \& Litvinov 2013b). The red blood cells give $45 \%$ of the volume of the blood, during clot formation. These are trapped within the fibrin fibres (Gersh et al. 2009), whilst the platelets are pulling or contracting on the fibrin fibres (Jen \& McIntire 1982; Lam et al. 2011). The presence of large volume of red blood cells restricts the normal fibrin network formation and platelet binding, and therefore, reduces the level of strain stiffening in LAOS (Shah \& Janmey 1997; Gersh et al. 2009; Jansen et al. 2013).

For all blood clot samples, intracycle stress stiffening of the material was observed, as $J_{M}^{\prime}$ dominated over $J_{L}^{\prime}$ in individual LAOStress experiments throughout the range of stress levels applied. The aforementioned regions shifted to different levels of stresses and strains for blood clots formed with different values of $d_{f}$. In clots formed with relatively low $d_{f}$ values $\left(d_{f}=1.48 \pm 0.024\right)$, produced by the addition of heparin, four different regions can be seen. In Region 1, the linear elastic compliance gradually increases with increasing stress, suggesting irreversible structural damage of the sample, which might be due to the stretching of the fibrin fibres being restricted by the cells captured within the fibrin network. $J_{M}^{\prime}$ also increases in this region, while $J_{L}^{\prime}$ is at a constant value or only very slightly decreasing. Linear elastic 
compliance, $J^{\prime}$, and nonlinear compliance, $J_{M}^{\prime}$, further increase in Region 2, whilst $J_{L}^{\prime}$ decreases with increasing stress. In Region 3, the blood clot displays stress stiffening behaviour, as $J_{M}^{\prime}$ remains constant and $J_{L}^{\prime}$ slightly decreases or remains constant with increasing stress. However, the consecutive SAOS measurements within this region show that the structure of the clot is becoming more damaged, as the linear elastic compliance continues to increase. Despite the damage to the clot, the network maintains such remarkable stress stiffening properties, which might help to resist further damage by increasing levels of stress. In the final, fourth region complete fracture of the clot occurs, which is indicated by a dramatic increase in the oscillation strain.

Higher values of $d_{f}\left(d_{f}=1.66 \pm 0.037\right)$ were measured for whole blood clots compared to heparinised blood clots, which coincided in shifting of the regions to lower levels of stresses and strains ranges, and to the disappearance of Region 3. Whilst, in the case of relatively high $d_{f}$ values $\left(d_{f}=1.76 \pm 0.032\right)$, as created by the addition of thrombin, only two regions can be identified. The behaviour seen in Region 2 and Region 3 in heparinised clots cannot be seen in the more dense clots formed with thrombin, and complete fracture of the clot takes place following Region 1.

The values of $d_{f}$ correlated with the eventual fracture stress of the blood clots. Increases in the $d_{f}$ resulted in decrease in the stress level at which point full clot fracture occurred. This overall trend in fracture stress versus structure (i.e. $\left.d_{f}\right)$ is similar to what was seen for fibrin clots (See Chapter 5.4.6), which might be expected as the fibrin network dominates the mechanical response to imposed stress. The highest value of fracture stress $(700 \mathrm{~Pa})$ was measured for clots formed with the lowest recorded $d_{f}\left(d_{f}=1.45\right)$. These clots might be described as "stable" insofar that they do not fracture easily, and would stay in the blood vessel, such as those seen in patients with DVT only. The lowest value of fracture stress (200 Pa) was measured for clots formed with the highest $d_{f}\left(d_{f}=1.80\right)$. These clots might be described as "unstable" because they are more prone to fracture, and could lead to an embolic event. Interpolation of the relationship between $d_{f}$ and fracture stress gave a prediction of zero fracture for $d_{f}$ of 1.9 , which is in good correlation with the LAOStrain findings (See Chapter 6.4.1). This is an interesting finding because the 
highest value of $d_{f}$ found in clinical studies of diseased blood is 1.88 (seen in stroke patients) (Stanford et al. 2015). Hence, a $d_{f}$ of 1.9 might be a limiting value for clots formed under SAOS.

Incipient clots with increasing values of $d_{f}$ led to mature clots with lower fracture stress values and a shift in the nonlinear regions to lower stresses and strains, which eventually lead to the disappearance of some of the regions at higher values of $d_{f}$. This observation could be important in understanding the etiology of embolism, as the clots with fewer nonlinear regions, and with regions at lower stress levels, could be more likely to break due to the forces within the blood vessels. Only two different nonlinear regions were detected for blood clots formed with $d_{f}=1.76 \pm 0.032$, and such clots are potentially more likely to embolise. A higher value of $d_{f}$ leads to a more dense structure containing thinner fibrin fibres, with more branch points; as a result, these clots are less permeable. These clots are also more resistant to the body's natural ability to break them down by fibrinolysis (Li et al. 2016), and therefore may exist in blood vessels for relatively long period of times. A combination of the decreased fracture stress and resistance to fibrinolysis makes these clots potentially more dangerous if formed within the blood vessels.

SEM images of clots with heparin and thrombin corroborated the results obtained from the rheological measurements of the Gel Point insofar that the underlying microstructures are distinct. The presence of thrombin resulted in a more dense fibrin network with more branch points, similar to clots encountered in thromboembolic disease (Ariens 2009); while clots formed in the presence of heparin display more open, more permeable structure, with thick fibres. This suggests that the observed shifting of the regions could be a consequence of the available free space within the clot. In the case of clots formed with a high $d_{f}$, or a more dense network, there is less free volume present. Therefore, as the clot is probed within the nonlinear region, the onset of stress stiffening appears earlier on, possibly due to that the movement of the fibres being restricted by the entrapped cells or even adjacent fibres. Those fibres that come in contact will stick together possibly due to the lateral aggregation of protofibrils (Weisel \& Litvinov 2013b; van Rooy et al. 2015). In contrast, in the case of lower $d_{f}$, and more open clot structure, due to the increased volume in pore space, there might be more opportunity for moving or pushing around the cells, as the fibrin 
fibre is being stretched or trying to rearrange in order to adapt to the applied large stresses. Therefore, these clots with lower $d_{f}$ values might show more nonlinear regions, in comparison to blood clots formed with a higher $d_{f}$ value.

In the final stage of the study, LAOStress properties of whole blood clots formed under CSPS were investigated. The relevance of this study is that the blood clot formation is known to be affected by the flow of blood within the blood vessels, and influences the forming blood clot structure. As a blood clot is forming within the vessel, the fibres align in the direction of the flow; hence the blood flow affects the structure of the clot, which determines its mechanical and chemical properties (Weisel \& Litvinov 2013b; Gersh et al. 2010; Campbell et al. 2010; Whittaker \& Przyklenk 2009; Varju et al. 2011). CSPS allows the study of blood clots formed under unidirectional shear flow at physiologically and pathologically relevant levels of shear stress (Badiei et al. 2015; Curtis et al. 2015). The $d_{f}$ value of the blood clots formed under unidirectional flow shifts to higher values (with highest recorded $d_{f}$ of 2.1369), compared to the clots formed under SAOS. These results are in good agreement with previous studies that performed Gel Point measurements on fibrin gels formed under CSPS (Badiei et al. 2015; Curtis et al. 2015), insofar that increases in the unidirectional shear flow component leads to an increase in $d_{f}$. The effect of CSPS on the process of clot formation, such as increased $d_{f}$ of incipient clot structures, can be explained by an increase in the monomer activation induced by the mixing in shear flow (Curtis et al. 2011; Curtis et al. 2015). Considering that the fractal microstructure of the incipient clot is the template for the structure of the fully formed, mature clot, and that even small increase in $d_{f}$ results in large amount of additional mass to the clot, the clots formed under CSPS are more dense and less permeable than clot formed under SAOS (Curtis et al. 2013).

The previously identified and described nonlinear regions in blood clots formed under SAOS can also be observed in clots formed under CSPS. In addition, a similar pattern of shifting in the regions, region disappearance, and change in fracture stress with different values of $d_{f}$ observed for blood clots formed under unidirectional flow. The aforementioned four different nonlinear regions can be detected for CSPS clots formed at lower $d_{f}$ values $\left(d_{f} \leq 1.95\right)$, whilst only two different regions (Region 1 and 4) were found for CSPS clots with higher $d_{f}$ values $\left(d_{f} \geq 2.00\right)$. The oscillatory stress 
and strain ranges of the different regions have shifted to lower values as increasing $d_{f}$ values were measured. Consequently, increasing values of $d_{f}$ resulted in decreasing fracture stress levels. By fitting a linear trendline to the fracture stress against $d_{f}$ dataset, $d_{f}$ of 2.15 was identified for zero fracture stress, which suggests a limiting value for clot formation under unidirectional flow, and could be representative of diseased clots formed in vivo.

\subsection{Conclusion}

The main conclusion of the work described in the present chapter pertaining to LAOS studies of whole blood clots is that clot microstructure, as measured by the rheologically derived biomarker $d_{f}$, predicts the eventual fracture stress of the clot. Therefore, the measurement of $d_{f}$ might serve as a much needed biomarker for predicting embolic events in patients whom experience DVT or other clotting events. 


\section{Conclusions and recommendations for further work}

This thesis presents an investigation into the nonlinear viscoelastic properties of fibrin and blood clots, in an attempt to explore mechanisms of embolism, i.e. fracture as a result of haemodynamic forces present within the blood vessels, and the role of clot microstructure. Advanced rheometrical techniques involving Large Amplitude Oscillatory Shear (LAOS) were applied in both strain-controlled (LAOStrain) and stress-controlled (LAOStress) modes for the nonlinear viscoelastic characterisation of fibrin and blood clots.

Fully formed, i.e. "mature", fibrin clots, that provide the underlying 3-dimensional microstructural component of blood clots, were characterised using LAOStrain as a model system for clotting. Fibrin clots were formed from $4 \mathrm{mg} / \mathrm{ml}$ fibrinogen, which is the upper limit of the normal physiological range of fibrinogen, and with different incipient clot microstructures as manipulated by changing concentration of thrombin. Relatively high thrombin concentrations create a more dense network consisting of thinner fibres and more branch points, whilst a relatively low thrombin concentration results in a more open or porous network consisting of thicker fibres with fewer branch points (Weisel \& Litvinov 2013b). In initial strain sweep experiments, the thrombin concentration did not show an effect on the eventual fracture of the fibrin network. The fracture stress was markedly decreased in the presence of FXIII inhibitor that prevents fibrin crosslinking. However, similar to the crosslinked fibrin clots, the fracture stress was unaffected by the thrombin concentration. This suggests that clots at an early stage of their formation (i.e. immediately following incipiency), whereby only a small amount of crosslinking is present, might be more prone to fracture.

A procedure was developed for testing the ability of fibrin network to adapt to the imposed deformation. This procedure used alternating LAOStrain and SAOS measurements, with increasing strain magnitudes applied in the LAOStrain tests. The experiment captured the remarkable strain stiffening properties of fibrin clots as seen in other studies (Janmey et al. 1983; Bale \& Ferry 1988; Shah \& Janmey 1997; Liu et al. 2006; Liu et al. 2010; Jansen et al. 2013; Münster et al. 2013; van Kempen et 
al. 2015). Moreover, it revealed several different regions within the nonlinear viscoelastic region of the material, which showed reversible and irreversible behaviour. This is possibly due to fibrin fibre stretching and bundling, yielding of branch points, structural rearrangements or localised fracture of the fibres and identification of these regions are important for understanding the etiology of embolism.

During LAOStress experiments, ringing was observed in both stress and strain waveforms. This is the first observation of ringing behaviour in a LAOStress experiment. The ringing was eliminated by a smoothing spline fit, in order to calculate nonlinear compliances. A modified ringing analysis was performed for evaluating the viscoelastic properties using the ringing behaviour within the LAOStress waveform. The results were in good agreement with those found using a ringing analysis of creep measurements of the same sample suggesting that the ringing was linked to a material property and is likely to be due to a coupling of inertia and elastic forces in the measurement i.e. inertio-elastic ringing. Nonlinear properties of fibrin clots were studied with the application of alternating SAOS and increasing levels of LAOStress steps. The stress stiffening behaviour of fibrin clots was captured in the measurements. The results showed different nonlinear viscoelastic regions, similar to those observed in LAOStrain experiments. Structural arrangements of the fibrin clot network that had reached a steady state within a region was imaged using SEM. The images provided confirmation of different degrees of fibre bundling, stretching, structural rearrangements and localised fracture, in the different regions.

The underlying microstructure of the fibrin clots was altered by the addition of various thrombin concentrations, which resulted in a shifting of the established regions to different levels of stress and a different value of fracture stress. Clots formed with higher thrombin concentration, hence with more dense network were found to be less resistant to the repeated increasing levels of stressing. Forming clots under CSPS also affected the nonlinear properties, in such way that increasing levels of unidirectional stress resulted in a decrease in fracture stress and a concomitant shift in the regions. 
The role of whole blood clot microstructure and its nonlinear rheological properties was investigated. The structure of the blood clots was quantified in a Gel Point measurement in terms of fractal dimension, whilst the nonlinear viscoelastic properties were characterised in LAOStrain and LAOStress measurements. The rheologically derived fractal dimension provided a means of quantifying incipient clot microstructure (Evans et al. 2010). This acts as a template for the ensuing clot development and predicts the structure of the mature clot (Curtis et al. 2013). The results showed a strong correlation between fractal dimension and fracture stress, with clots formed with higher values of fractal dimension eventually fracturing at a lower level of stress. Both LAOStrain and LAOStress experiments have predicted a fractal dimension of 1.9 for zero fracture stress, providing a limiting value for blood clot formed under SAOS. This value is commensurate with those seen using blood from prothrombotic patients including Stroke, Cancer, and some (warfarinised) patients with DVT. This suggests that some patients are still at risk of an embolic event (a PE) despite being fully anticoagulated according to their value of International Normalised Ratio (INR), a common laboratory assay based on the prothrombin time.

In conclusion, the results presented suggest that the measurement of fractal dimension of whole blood clots is a biomarker for predicting embolic events in patients with abnormal coagulopathies. Patients whom experience clots formed with high values of fractal dimension are also more likely to experience an embolic event. Patients whom experience clots formed with a low value of fractal dimension are more likely to have a stable clot that resides in the vessels and does not embolise e.g. DVT only. Thus, the measurement of a fractal dimension from blood obtained from such patients may inform clinical management and therapeutic intervention.

A limitation of this work is that it is a relatively small scale laboratory study. A large scale clinical study should be conducted in order to explore the nonlinear viscoelastic properties of clots formed from blood taken from people suffering from VTE using the method proposed herein. The alternating LAOStress and SAOS technique alongside with Gel Point detection can allow researchers or clinicians to test VTE patient's blood. Results should be gathered and monitored, i.e. cases of DVT only or DVT and PE with the corresponding fractal dimension, regions and fracture stress 
data over time to build up a database, which may help identifying a range of fractal dimensions in which individuals have a tendency for embolism. Furthermore, a prospective observational study in patients with DVT only will provide data in order to evaluate the ability of fractal dimension to predict those whom eventually go on to experience an embolism.

Rheometry could also be used in anticoagulant and thrombolytic drug development. The techniques presented herein involving alternating LAOStress and SAOS technique could be used in assessing the efficacy of novel anticoagulant or thrombolytic drugs, or for studying the effect of pre-existing anticoagulant medications admitted to the patients. Whilst it is important to breakdown clots using thrombolytics, overuse of such drugs has harmful side effects like embolism and bleeding events. Measurement of the efficacy of these therapies to a patient's sample of blood opens up the avenue for personalised medicine. Novel anticoagulant medication could be developed that modifies clots so that they are more resistant to fracture and more susceptible to fibrinolysis whilst maintaining normal haemostasis. These vital factors that underpin coagulopathies can be measured using the techniques described and developed herein which provide a detailed analysis of mechanical (nonlinear viscoelastic) and structural properties of the blood clot. 


\section{Appendices}

\subsection{Published work}

PHYSICS OF FLUIDS 29, 121606 (2017)

\section{The application of large amplitude oscillatory stress in a study of fully formed fibrin clots}

T. F. Lamer, ${ }^{1}$ B. R. Thomas, ${ }^{1}$ D. J. Curtis ${ }^{2}$ N. Badiei, ${ }^{2}$ P. R. Williams,${ }^{2}$ and K. Hawkins ${ }^{1, a}$

${ }^{1}$ Swansea University Medical School, Swansea University, Swansea SA2 8PP, United Kingdom

${ }^{2}$ Complex Fluids Research Group, College of Engineering, Swansea University, Swansea SA1 8EN,

United Kingdom

(Received 12 August 2017; accepted 13 September 2017; published online 27 October 2017)

The suitability of controlled stress large amplitude oscillatory shear (LAOStress) for the characterisation of the nonlinear viscoelastic properties of fully formed fibrin clots is investigated. Capturing the rich nonlinear viscoelastic behaviour of the fibrin network is important for understanding the structural behaviour of clots formed in blood vessels which are exposed to a wide range of shear stresses. We report, for the first time, that artefacts due to ringing exist in both the sample stress and strain waveforms of a LAOStress measurement which will lead to errors in the calculation of nonlinear viscoelastic properties. The process of smoothing the waveforms eliminates these artefacts whilst retaining essential rheological information. Furthermore, we demonstrate the potential of LAOStress for characterising the nonlinear viscoelastic properties of fibrin clots in response to incremental increases of applied stress up to the point of fracture. Alternating LAOStress and small amplitude oscillatory shear measurements provide detailed information of reversible and irreversible structural changes of the fibrin clot as a consequence of elevated levels of stress. We relate these findings to previous studies involving large scale deformations of fibrin clots. The LAOStress technique may provide useful information to help understand why some blood clots formed in vessels are stable (such as in deep vein thrombosis) and others break off (leading to a life threatening pulmonary embolism). (c) 2017 Author(s). All article content, except where otherwise noted, is licensed under a Creative Commons Attribution (CC BY) license (http://creativecommons.org/licenses/by/4.0/). https://doi.org/10.1063/1.4999991

\section{INTRODUCTION}

Characterisation of the nonlinear rheological properties of biopolymer gels has important implications for understanding their structure-function relationships under large stress and strain regimes. A salient example is a fibrin gel that provides the structural backbone of a blood clot and serves to perform a haemostatic function by preventing the loss of blood at a site of injury to a vessel. ${ }^{1}$ A fibrin gel is formed following the conversion of fibrinogen to fibrin by the action of the catalytic enzyme thrombin and subsequent polymerisation of fibrin to produce a three-dimensional gel network of branching fibres. ${ }^{1}$ The fibres are formed from protofibrils that may aggregate both laterally and longitudinally. Conditions that favour lateral aggregation produce thicker fibres with relatively low degree of branching, while conditions that inhibit lateral aggregation yield thin fibres with more branching. ${ }^{1}$ The evolving fibrin network entraps cells such as red blood cells, white blood cells, and platelets and is reinforced by Factor XIII mediated covalent cross-linking to produce a more mechanically and chemically stable fully formed fibrin clot. ${ }^{2-4}$ In disease, the undesirable formation of fibrin clots can block blood vessels, thus preventing blood supply to tissues. Blockages in the veins are termed a Venous Thromboembolism (VTE) and are responsible for a significant global disease burden. ${ }^{5}$

a) Author to whom correspondence should be addressed: k.m.hawkins@ swansea.ac.uk

$1070-6631 / 2017 / 29(12) / 121606 / 6$
Clots formed in the deep veins (i.e., a deep vein thrombosis) can embolise (i.e., break off), travel in the bloodstream, and lodge in the pulmonary arteries leading to a lack of blood supply to the lungs (i.e., a pulmonary embolism). ${ }^{6,7}$

In vivo, shear forces due to the flow of blood may induce fracture or breakage of the blood clot and is a potential mechanism for embolism. ${ }^{89}$ The wall shear stress in veins can span several magnitudes depending on the size, branching, and number of vessels and can be elevated in disease states. ${ }^{10}$ Microfluidic channel approaches studying clot growth have shown that, in a "pressure-relief mode" whereby the flow of blood is allowed to divert to other channels, the shear stress increases as the flow squeezes past the growing thrombus and then rapidly decreases at approximately $80 \%$ of full occlusion. ${ }^{9}$ Thus, it is important to consider the effects of both the vast range and progressive increase in stress experienced by fully formed clots. Marked changes in the structure and rheological properties of the clot can arise as a consequence of shear stress through several different mechanisms including strain-stiffening of the fibrin network, ${ }^{11}$ strain-stiffening of individual fibrin fibres, ${ }^{12,13}$ protein unfolding of stretched fibres ${ }^{14}$ unfolding of fibrin monomers ${ }^{15}$ yielding of branching points, ${ }^{16}$ slippage of protofibrils within fibres,${ }^{17}$ and breakage of individual fibrin fibres. ${ }^{18,19}$ The propensity of the formed blood clot to macroscopically fracture may be influenced by all of the aforementioned mechanisms.

There have been several methods used to characterise the rheological properties of fibrin clots under relatively large 
stresses and strains. These include conventional rheological approaches involving oscillatory techniques under conditions that provoke strain-stiffening behaviour of the network and the subsequent reporting of storage moduli. ${ }^{11,20}$ However, it is important to recognise that, under such conditions, the resulting waveform is non-sinusoidal and thus does not pertain to the theory of linear viscoelasticity. ${ }^{21}$ Other workers have exploited inertio-elastic oscillations in controlled stress rheometry to provide a measurement of tangent moduli. ${ }^{22}$ Several studies have reported the application of a pre-stress followed by the superposition of small amplitude oscillatory shear stress to probe the rheological properties in terms of differential moduli. ${ }^{15,23,24}$ Using this approach, Piechocka et al..$^{15}$ identified distinct regimes of elastic response. Large Amplitude Oscillatory Shear (LAOS) has been used in a controlled strain manner to perform repeated large strain loadings on fibrin clots and the data were presented using Lissajous-Bowditch curves. ${ }^{17,25}$ Using repeated cyclic strain loadings, Münster et $a{ }^{17}{ }^{17}$ showed the ability of un-crosslinked fibrin clots to adapt to stress through the slippage of protofibrils. Moreover, the LAOS studies of van Kempen et al. ${ }^{25}$ revealed several nonlinear features of fibrin clots, including time-dependent softening, intra-cycle strain stiffening, and increased viscous dissipation with increased strain, illustrating the usefulness of LAOS in capturing rich nonlinear behaviour.

Controlled stress LAOS (LAOStress) is arguably a more suitable technique than controlled strain LAOS for studying the nonlinear properties of fully formed fibrin clots. LAOStress is physiologically relevant insofar that it affords progressive increases in stress to be imposed on the sample which can be related to the hemodynamic forces experienced by clots in vivo. LAOStress has been previously used to characterise the nonlinear rheology of polymer solutions and gels. ${ }^{26,27}$ In the present study, we investigate the suitability of LAOStress for studying the nonlinear behaviour of fibrin clots up to the point of macroscopic fracture. Furthermore, we combine alternating LAOStress and linear viscoelastic measurements in order to study reversible and irreversible changes in fibrin clot microstructure as a consequence of different levels of stress.

\section{EXPERIMENTAL}

\section{A. Materials}

Purified, plasminogen depleted human fibrinogen at least 95\% clottable and human thrombin were obtained from Enzyme Research Laboratories, UK. The fibrinogen was made up to a stock solution of $44.54 \mathrm{mg} / \mathrm{ml}$ by the addition of Trisbuffer saline, TBS (20 mM Tris, $\mathrm{pH} 7.4 \%$ and $0.9 \% \mathrm{NaCl}$, Sigma Aldrich, UK) and left to fully dissolve in a water bath at $37^{\circ} \mathrm{C}$. Thrombin was dissolved in distilled water and made up to a stock solution of $500 \mathrm{NIH}$ unit $/ \mathrm{ml}$. These stock solutions were aliquoted and frozen at $-80{ }^{\circ} \mathrm{C}$ until required. A $1 \mathrm{M}$ stock solution of $\mathrm{CaCl}_{2}$ (Sigma Aldrich, UK) was also stored at $4{ }^{\circ} \mathrm{C}$. The appropriate volumes of TBS, fibrinogen, and $\mathrm{CaCl}_{2}$ were mixed together to make a (final) concentration of $4 \mathrm{mg} / \mathrm{ml}$ fibrinogen and $0.005 \mathrm{M} \mathrm{CaCl}_{2}$. Clotting was initiated by adding the required volume of thrombin to produce a (final) concentration of $0.1 \mathrm{NIH} / \mathrm{ml}$. After appropriate mixing, the sample was transferred onto the lower plate of the rheometer.

\section{B. Rheometry}

Rheological measurements were conducted using an AR G2 controlled stress rheometer (TA Instruments, UK) fitted with a cone and plate (40 $\mathrm{mm}$ cone, $0.0351 \mathrm{rad}$ ) measuring system. The lower plate was maintained at a temperature of $24{ }^{\circ} \mathrm{C}$ for all measurements. In order to reduce effects of evaporation, the sample was sealed with a thin layer of low viscosity (10 $\mathrm{mPa} \mathrm{s})$ silicone oil. The sample was allowed to clot between the rheometer plates for a period equivalent to 40 gel times, after which the network is fully formed. ${ }^{28,29}$ LAOStress measurements were performed on the fully forme clot by applying continuous stress waveforms at a stress amplitude in the nonlinear viscoelastic range of the sample and a frequency of $1 \mathrm{~Hz}$ until the sample reached a steady state. Following the acquisition of LAOStress data, the sample was probed by small amplitude oscillatory stress measurements at a stress level of $5 \mathrm{~Pa}$ and a frequency of $1 \mathrm{~Hz}$ until it reached steady state in order to provide a value of linear elastic compliance $J^{\prime}$. Adherence of the Small Amplitude Oscillatory Shear (SAOS) measurements to the linear viscoelastic range of the sample was confirmed by the absence of a third harmonic response. The process of alternating LAOStress measurement at incrementally increasing stress amplitudes and SAOS measurements was performed until the sample macroscopically fractured leading the upper rheometer plate to overspeed. A criterion was imposed throughout all experiments that steady state was reached once the difference in the strain response between consecutive waveforms was less than $2 \%$. This criterion ensured that point symmetry was observed in the Lissajous-Bowditch curves obtained from the LAOStress test and that irreversible damage following relaxation of the sample was probed by SAOS. The sample stress and strain response waveform datasets were obtained by the rheometer software (TRIOS) in a transient data collection mode at the software's default sampling rate of $978 \mathrm{~Hz}$

\section{Analysis of LAOStress waveforms}

A quantitative framework for the analysis of LAOStress data in terms of deducing the nonlinear compliances has been described by Dimitriou et al. ${ }^{26}$ and Läuger and Stettin. ${ }^{27}$ This includes the minimum-stress elastic compliance $J_{M}^{\prime}$ and the large-stress elastic compliance $J_{L}^{\prime}$ (see Fig. 1) which can be calculated from Lissajous-Bowditch curves of strain versus stress. ${ }^{26}$

For an imposed sinusoidal stress, $\sigma(t)=\sigma_{0} \cos \omega t$, the minimum-stress elastic compliance, $J_{M}^{\prime}$, can be defined by

$$
\begin{aligned}
J_{M}^{\prime} & \left.\equiv \frac{d \gamma}{d \sigma}\right|_{\sigma=0}=\sum_{n \text { odd }}(-1)^{(n-1) / 2} n J_{n}^{\prime} \\
& =\sum_{n \text { odd }}(-1)^{(n-1) / 2} n c_{n}
\end{aligned}
$$

and the large-stress elastic compliance, $J_{L}^{\prime}$, can be defined by

$$
\left.J_{L}^{\prime} \equiv \frac{\gamma}{\sigma}\right|_{\sigma=\sigma_{0}}=\sum_{n o d d} J_{n}^{\prime}=\sum_{n o d d} c_{n},
$$

where $\sigma_{0}$ is the applied stress amplitude, $\gamma$ is the resulting strain, $c_{n}$ is the $n$th order Chebyshev coefficient, in which the 


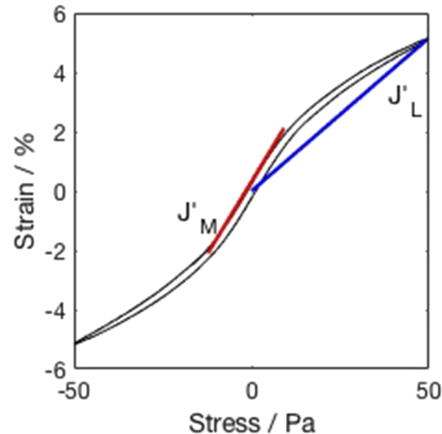

FIG. 1. A Lissajous-Bowditch curve obtained from a LAOStress experiment and an illustration of the calculation of nonlinear compliances, the minimumstress elastic compliance $J_{M}^{\prime}\left(\right.$ red) and the large-stress elastic compliance $J_{L}^{\prime}$ (blue).

letter "c" represents the compliance nonlinearities, and $J_{n}^{\prime}$ is the $n$th compliance. ${ }^{26}$

Figure 1 shows an illustration of a Lissajous-Bowditch curve of stress versus strain data obtained from a LAOStress measurement. $J_{M}^{\prime}$ is calculated from the gradient of the tangent at zero stress. $J_{L}^{\prime}$ is calculated from the gradient of a straight line connecting the origin to the point of maximum stress. The calculations were performed using MATLAB (Version 9.2, MathWorks, Inc.) after importing data from TRIOS.

\section{RESULTS AND DISCUSSION}

\section{A. LAOStress data processing}

Figure 2 displays an example of sample stress waveforms (upper) and strain response waveforms (lower) at an applied stress level of (a) $100 \mathrm{~Pa}$ (slightly into the nonlinear viscoelastic range of the sample) and two higher levels of applied stress (b) $500 \mathrm{~Pa}$ and (c) $1500 \mathrm{~Pa}$. The dimensionless inertia number, In, proposed by Dimitriou et al. ${ }^{26}$ was calculated for all LAOStress measurements and $I n \ll 0.05$ throughout, indicating that instrument inertia alone did not significantly affect the total torque signal. However, at increasing levels of applied stress, the sample stress and strain response waveforms contain artefacts due to a source of ringing. To our knowledge, this is the first observation of ringing phenomena in a LAOS measurement. This ringing is possibly attributable to a resonance caused by a coupling of inertial and elastic forces at relatively large levels of applied stress. ${ }^{30}$

In an attempt to eliminate the effects of ringing, the sample stress and strain response waveform datasets (obtained from TRIOS) were smoothed to a cubic spline function (specifically a piecewise cubic polynomial computed from a smoothing parameter $\mathrm{p}=0.99999252$ ) using the Curve Fitting Toolbox in MATLAB. The datasets were then reconstructed from the smoothing function by sampling at a rate identical to the original dataset $(978 \mathrm{~Hz})$. Figure 3 displays an example of the resulting smoothed waveforms. The process of smoothing eliminates any noticeable effects of ringing. However, in order to ensure that nonlinearities due to the sample response (which manifest as odd harmonics) are not affected by the process of smoothing, the datasets were subjected to Fourier analysis and the resulting frequency spectra were compared. To this end, the strain and stress datasets were trimmed to contain 15000 data points which contain more than 15 complete cycles. The datasets were windowed using a Hanning window function and zero padded with 5000 data points in order to adjust the resolution of the frequency spectrum to $0.05 \mathrm{~Hz}$. The datasets were analysed in MATLAB using a discrete Fourier transform.

Figure 4 shows the frequency spectra of the sample stres and strain response waveforms for the original (i.e., unprocessed) data and smoothed data at three different levels of applied stress (a) $100 \mathrm{~Pa}$, (b) $500 \mathrm{~Pa}$, and (c) $1500 \mathrm{~Pa}$. The frequency spectra of the strain response are dominated by the fundamental (imposed) frequency and contain odd harmonics, confirming that the measurement is within the nonlinea viscoelastic regime. ${ }^{21}$ The magnitude of the even harmonics
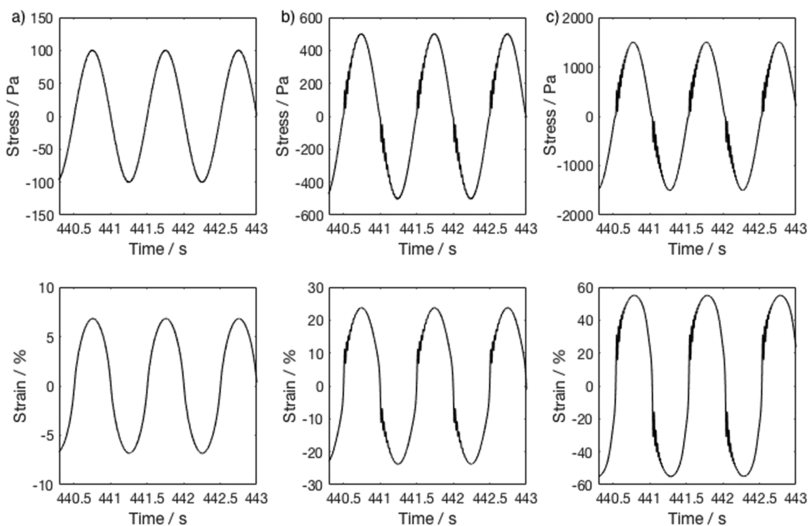

FIG. 2. Sample stress (upper) and strain response (lower) waveforms obtained from the rheometer (TRIOS) software at input stress levels of a) $100 \mathrm{~Pa}$, (b) $500 \mathrm{~Pa}$, and (c) $1500 \mathrm{~Pa}$ These waveforms consist of discre 

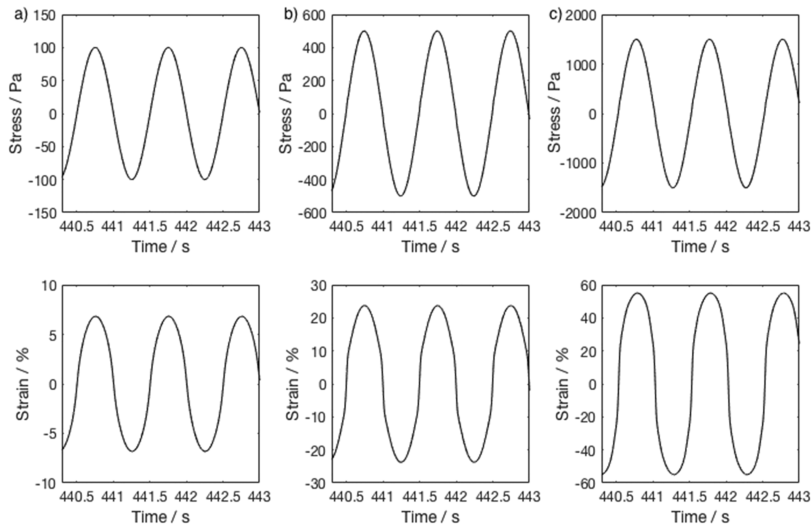

FIG. 3. Smoothed sample stres (upper) and strain response (lower) waveforms (corresponding to the waveforms shown in Fig. 2) at input stres levels of (a) $100 \mathrm{~Pa}$, (b) $500 \mathrm{~Pa}$, an (c) $1500 \mathrm{~Pa}$. These waveforms consis of discrete data points at a sampling rate of $978 \mathrm{~Hz}$

was negligible confirming that the sample was non-transient and had reached a steady state. ${ }^{31}$ It is noticeable that some harmonic distortion in the sample stress waveform is apparent, including at the lowest stress shown where there was no ringing encountered. These nonlinearities will be transmitted to, and replicated in the corresponding strain response. However, the magnitude of the odd harmonics in the strain response is much greater than the sample stress indicating that the rich nonlinearity of the sample is being captured. Importantly, the frequency spectra of the smoothed waveforms are identical to their original waveforms providing evidence that the process of smoothing does not eliminate the essential information originating from the nonlinear response of the sample. Henceforth, we use the smoothed stress and strain waveforms as the basis of the Lissajous-Bowditch curves and calculation of nonlinear compliances.

Figure 5 shows a comparison of Lissajous-Bowditch curves constructed from original and smoothed datasets.
At relatively low levels of stress [Fig. 5(a)], the LissajousBowditch curves appear identical. Figures 5(b) and 5(c) show that the ringing encountered in the original waveforms introduces secondary loops in the corresponding LissajousBowditch curves. These secondary loops are eliminated by the process of smoothing. Furthermore, at these relatively large stress levels, inspection of the original and smoothed curves shows profound differences around the region pertaining to the minimum stress. Thus, any calculated values of $J_{M}^{\prime}$ are highly sensitive to ringing artefacts.

\section{B. Nonlinear and linear viscoelastic properties}

Figure 6 shows the results of the alternating LAOStress and SAOS measurements. Figure 6(b) displays the magnitude of the strain response to the applied stress versus time profile shown in Fig. 6(a). The time period for which each stress is applied depends on the duration of the measurement to reach a steady state. Figure 6(c) shows that values of the nonlinear
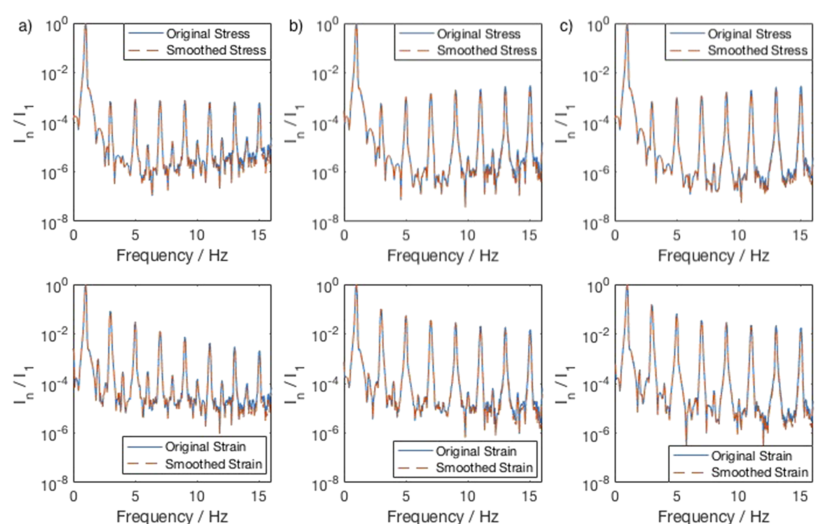

FIG. 4. Comparisons of frequency spectra obtained from discrete Fourie transforms of the original (blue) and smoothed (red) datasets for the sample stress waveform (upper) and strain response wavefors (lower) at inpu tress le 1500 Pa. of the spectra (II) a The nom of the specta $\left(I_{n}\right)$ are nomalised to he ro mental (imposed) frequency is 1 Hz throughout. 

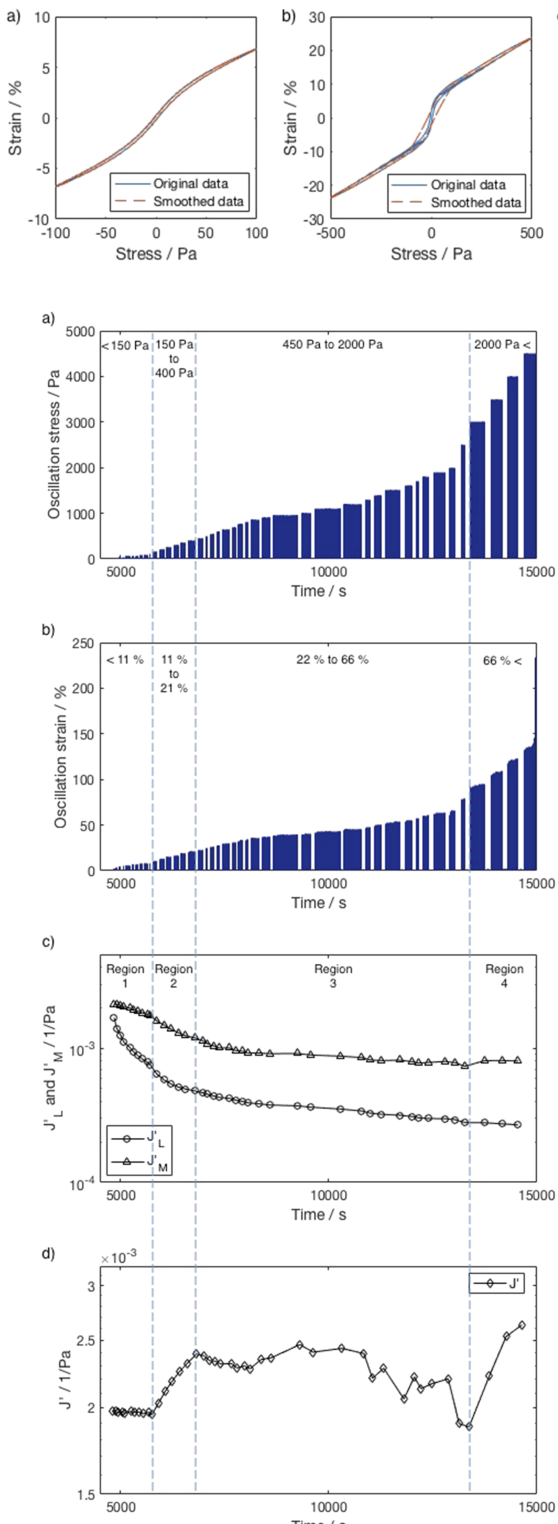

FIG. 6. Results of alternating SAOS and LAOStress measurements; (a) the applied stress versus time profile, (b) the resulting strain response versus time profile, (c) large-stress and minimum-stress elastic compliances (as measured by LAOStress), and (d) linear elastic compliance (as measured by SAOS). The results reveal several nonlinear viscoelastic regions (numbered $1-4$, as illustrated by the dashed lines).

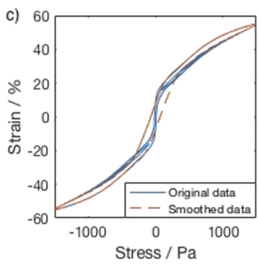

FIG. 5. Comparisons of LissajousBowditch curves using original (blue) and smoothed (red) datasets for input $100 \mathrm{~Pa}$ (b) $500 \mathrm{~Pa}$ and (c) $1500 \mathrm{~Pa}$.

compliances $J_{L}^{\prime}$ and $J_{M}^{\prime}$ gradually decrease with increasing levels of stress and strain indicating stress stiffening of the sample. This stress stiffening is also captured in individual LAOStress measurements at discrete levels of stress by a dominance of $J_{M}^{\prime}$ over $J_{L}^{\prime}$ over the entire range of stress levels studied. Thi behaviour is expected given that individual fibrin fibres' strain stiffens and is highly extensible ${ }^{12,13,15}$ Our findings are similar to the LAOS controlled strain studies of van Kempen et al. ${ }^{25}$ that reported intra-cycle stiffening and softening during continuous application of large strain deformations to fibrin clots. However, an advantage of LAOStress measurements is that the fibrin clot is allowed to reach the steady state under a fixed level of oscillatory stress. This is more physiologically relevant insofar that the stress is imposed on the clot. ${ }^{9,10}$

Analysis of the nonlinear and linear viscoelastic behaviour of the sample [in Figs. 6(c) and 6(d), respectively] reveals four distinct regions. In region $1\left(\sigma_{0}<150 \mathrm{~Pa}\right)$, the sample shows increasing tendency to stress stiffen as evidenced by an increase in the difference between $J_{M}^{\prime}$ and $J_{L}^{\prime}$ with increasing levels of stress. The sample returns to its original linear viscoelastic behaviour following a decrease in applied stress indicating full recovery of the structure. In region 2 $\left(150<\sigma_{0}<400 \mathrm{~Pa}\right)$, the linear elastic compliance gradually increased following the application of increasing levels of LAOStress, indicating irreversible structural rearrangements. This stress-softening, which is also evident by close inspection of the curvature of the oscillatory strain response in the LAOStress experiments, is likely to be due to yielding of branching points leading to a slackening of the network. ${ }^{16}$ The behaviour encountered in region $3\left(450<\sigma_{0}<2000 \mathrm{~Pa}\right)$ is similar to region 1 insofar that the sample returns to a level of linear elastic compliance that is independent of the stress applied in the LAOStress measurement. This finding is similar to the study of Piechocka et al. ${ }^{15}$ which showed that the differential modulus is independent of values of pre-stress within a specific stress regime. In region 3 , the structure of the sample has been permanently re-arranged by the stresses encountered in region 2, resulting in a sample that has the ability to resist structural changes when exposed to furthe increases in stress. Furthermore, in the latter stages of region 3 (i.e., at long times), there is generally a decrease in linear elastic compliance following the application of increasing levels of LAOStress, indicating that effects due to ageing of the sample (possibly due to Factor XIII mediated cross-linking) are greater than any stress induced structural damage. In region 4 $\left(\sigma_{0}>2000 \mathrm{~Pa}\right)$, the ability of the sample to resist structural changes at increasing levels of stress diminishes as evidenced by an increase in linear elastic compliance. Further increase 
in the applied levels of stress lead to fracture of the sample network causing the rheometer upper plate to overspeed.

\section{CONCLUSIONS}

We investigated the applicability of LAOStress in studying the nonlinear viscoelastic properties of fully formed fibrin clots. The stress and strain waveforms obtained from the LAOStress measurements contained artefacts due to a source of ringing. This is the first observation of ringing in a LAOS measurement. We showed that the artefacts due to ringing can be effectively eliminated whilst retaining the essential harmonic information related to the nonlinear viscoelastic response of the sample by fitting the original waveforms to a smoothing spline. This has allowed a characterisation of the nonlinear compliances of fibrin clots up to the point of fracture. Combined with supplementary SAOS measurements, various regions of nonlinear viscoelastic behaviour were identified which can be related to previous findings involving large scale deformations of fibrin clots. The quantitative framework of LAOStress provides additional information of fibrin clot nonlinear behaviour, and the approach is more physiologically relevant than LAOS in a controlled strain mode. Further work will involve the application of LAOStress in studies of abnormal clots such as those formed in blood taken from patients with venous thromboembolism.

\section{ACKNOWLEDGMENTS}

This work was supported by a Health and Care Research Wales Studentship (HS-14-69) and the Engineering and Physical Sciences Research Council (EP/N013506/1). We would like to thank Gareth McKinley for the insightful discussions on the analysis of the LAOStress waveforms.

${ }^{1}$ J. W. Weisel, "The mechanical properties of fibrin for basic scientists and clinicians," Biophys. Chem. 112, 267 (2004).

${ }^{2}$ N. A. Kurniawan, J. Grimbergen, J. Koopman, and G. H. Koenderink, "Factor XIII stiffens fibrin clots by causing fiber compaction," J. Thromb. Haemostasis 12, 1687 (2014)

${ }^{3} \mathrm{~J}$. W. Weisel, "Mechanisms of fibrin polymerization and clinical implications," Blood 121, 1712 (2013).

${ }^{4}$ J. W. Weisel and R. I. Litvinov, "Fibrin Formation, Structure and Properties," Subcell. Biochem. 82, 405 (2017).

${ }^{5}$ ISTH Steering Committee for World Thrombosis Day, "Thrombosis: a major contributor to the global disease burden," J. Thromb. Haemostasis 12, 1580 (2014)

${ }^{6}$ J. E. Dalen, "Pulmonary embolism: what have we learned since Virchow? Natural history, pathophysiology, and diagnosis," Chest 122, 1440 (2002). ${ }^{7}$ K. R. Machlus, M. M. Aleman, and A. S. Wolberg, "Update on venous thromboembolism: risk factors, mechanisms, and treatments," Arterioscler., Thromb., Vasc. Biol. 31, 476 (2011).

${ }^{8}$ W. W. Roberts, O. Kramer, R. W. Rosser, F. H. Nestler, and J. D. Ferry, "Rheology of fibrin clots. I. Dynamic viscoelastic properties and fluid permeation," Biophys. Chem. 1, 152 (1974).

${ }^{9}$ T. V. Colace, R. W. Muthard, and S. L. Diamond, "Thrombus growth and embolism on tissue factor-bearing collagen surfaces under flow: role of thrombin with and without fibrin," Arterioscler., Thromb. Vasc. Biol. 32, $1466(2012)$

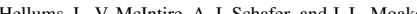
"Platelets and Shear Stress" Blood 88, 1525 (1996).

J. V. Sha and P. A. Jans," "Strin hardening of fibrin gels and plasma clots," Rheol. Acta 36, 262 (1997).

clots," Rheol. Acta 36, 262 ('S7). O'Brien R. M. Taylor, R. Superfine,

S. T. Hodson, J. R. Houser, E. T. O'Brien, R. M. Taylor, R. Superfine, S. T. Lord, and M. R. Falvo, "Stiffening of individual fibrin fibers equi(2010)

${ }^{13}$ W. Liu, C. R. Carlisle, E. A. Sparks, and M. Guthold, "The mechanical properties of single fibrin fibers." J. Thromb. Haemostasis 8, 1030 (2010). ${ }^{4} \mathrm{~K}$. P. Purohit, R. I Litvinov, A. E. X Brown, D. E. Discher and J.W. Weisel, "Protein un Protein unfolding accounts for the unusual

${ }^{15}$ I. K. Piechock R. G. Back c. Potters, F. C. MacKintosh, and G. H. Koenderink, "Structural hierarchy governs fibrin gel mechanics," Biophys. J. 98, 2281 (2010).

${ }^{16}$ W. Liu, L. Jawerth, E. Sparks, M. Falvo, R. Hantgan, R. Superfine, S. T. Lord, and M. Guthold "Fibrin fibers have extraordinary extensibility and elasticity," Science 313, 634 (2006).

${ }^{17}$ S. Münster, L. M. Jawerth, B. A Leslie, J. I. Weitz, B. Fabry, and D. A. Weitz "Strain history dependence of the nonlinear stress response of fibrin and collagen networks," Proc. Natl. Acad. Sci. U. S. A. 110, 12197 (2013).

${ }^{18}$ A. E. Brown, R. I Litvinov, D. E. Discher, P. K. Purohit, and J W. Weisel,

"Multiscale mechanics of fibrin polymer: gel stretching with protein unfolding and loss of water," Science 325, 741 (2009).

${ }^{19} \mathrm{~N}$. Badiei, A. M. Sowedan, D. J. Curtis, M. R. Brown, M. J. Lawrence, A. I. Campbell, P. A. Evans, J. W. Weisel, I. N. Chernysh, C. Nagaswami, P. R. Williams, and K. Hawkins, "Effects of unidirectional flow shea stresses on the formation, fractal microstructure and rigidity of incipien whole blood clots and fibrin gels," Clin. Hemorheol. Microcirc. 60, 451 (2015).

${ }^{20}$ P. Riha, X. Wang, R. Liao, and J. F. Stoltz, "Elasticity and fracture strain of whole blood clots." Clin. Hemorheol. Microcirc. 21, 45 (1999).

${ }^{21}$ A. J. Giacomin and J. M. Dealy "Large amplitude oscillatory shear" in Techniques in Rheological Measurement, edited by A. A. Collyer (Champan and Hall, London, 1993).

${ }^{22} \mathrm{~N}$. Y. Yao, R. J. Larsen, and D. A. Weitz, "Probing nonlinear rheology with inertio-elastic oscillations," J. Rheol. 52, 1013 (2008).

${ }^{3}$ C. P. Broedersz, K. E. Kasza, L. M. Jawerth, S. Munster, D. A. Weitz, and F. C. MacKintosh, "Measurement of nonlinear rheology of cross-linked biopolymer gels," Soft Matter 6, 4120 (2010).

${ }^{24}$ K. A. Jansen, R. G. Bacabac, I. K. Piechocka, and G. H. Koenderink, "Cells actively stiffen fibrin networks by generating contractile stress," Biophys. J. 105, 2240 (2013)

${ }^{25}$ T. H. S. van Kempen, G. W. M. Peters, and F. N. van de Vosse, "A constitutive model for the time-dependent, nonlinear stress response of fibrin networks," Biomech. Model. Mechanobiol. 14, 995 (2015).

${ }^{26}$ C. J. Dimitriou, R. H. Ewoldt, and G. H. McKinley, "Describing and prescribing the constitutive response of yield stress fluids using large amplitude oscillatory shear stress (LAOStress)," J. Rheol. 57, 27 (2013).

${ }^{27} \mathrm{~J}$. Läuger and H. Stettin, "Differences between stress and strain control in the non-linear behavior of complex fluids," Rheol. Acta 49, 909 (2010).

${ }^{28}$ F. Ferri, M. Greco, G. Arcovito, F. Bassi, M. De Spirito, E. Paganini, an M. Rocco, "Growth kinetics and structure of fibrin gels," Phys. Rev. 63, 31401 (2001)

D. J. Curtis, K. Hawkins, M. Lawrence, T. M. Davies, M. R. Brown, and P. R. Williams, "Incipient fibrin gel microstructure as an indication of maturing clot growth processes," Ser. Biomech. 25, 184 (2010).

${ }^{30}$ C. Baravian, G. Benbelkacem, and F. Caton, "Unsteady rheometry: can we characterize weak gels with a controlled stress rheometer?," Rheol. Acta 46, 577 (2007).

${ }^{31} \mathrm{~K}$. Atalik and R. Keunings, "On the occurrence of even harmonics in the shear stress response of viscoelastic fluids in large amplitude oscillatory shear," J. Non-Newtonian Fluid Mech. 122, 107 (2004). 


\section{Authorship statement}

\section{Declaration:}

The following people and institutions contributed to the publication of work undertaken as part of this thesis:

\begin{tabular}{|l|l|}
\hline Candidate & $\begin{array}{l}\text { Tunde Fruzsina Lamer } \\
\text { Swansea University Medical School, Swansea University }\end{array}$ \\
\hline Author 1 & $\begin{array}{l}\text { Tunde Fruzsina Lamer } \\
\text { Swansea University Medical School, Swansea University }\end{array}$ \\
\hline Author 2 & $\begin{array}{l}\text { Bethan R. Thomas } \\
\text { Swansea University Medical School, Swansea University }\end{array}$ \\
\hline Author 3 & $\begin{array}{l}\text { Daniel J. Curtis } \\
\text { College of Engineering, Swansea University }\end{array}$ \\
\hline Author 4 & $\begin{array}{l}\text { Nafiseh Badiei } \\
\text { College of Engineering, Swansea University }\end{array}$ \\
\hline Author 5 & $\begin{array}{l}\text { P. Rhodri Williams } \\
\text { College of Engineering, Swansea University }\end{array}$ \\
\hline Author 6 & $\begin{array}{l}\text { Karl Hawkins } \\
\text { Swansea University Medical School, Swansea University }\end{array}$ \\
\hline
\end{tabular}

\section{Author details and their roles:}

Paper 1: The application of large amplitude oscillatory stress in a study of fully formed fibrin clots

Located in Chapter 5

Candidate contributed to experimental work, data analysis and writing the paper. Percentage estimate of contribution: $68 \%$ 
Author 1 (i.e. Candidate) contributed to experimental work, data analysis and writing the paper. Percentage estimate of contribution: $68 \%$

Author 2 contributed to experimental work. Percentage estimate of contribution: $5 \%$

Author 3 contributed to data analysis. Percentage estimate of contribution: $5 \%$

Author 4 contributed to experimental work. Percentage estimate of contribution: $5 \%$

Author 5 contributed to writing the paper. Percentage estimate of contribution: $5 \%$

Author 6 contributed to writing the paper. Percentage estimate of contribution: $12 \%$

We the undersigned agree with the above stated "proportion of work undertaken" for each of the above published peer-reviewed manuscripts contributing to this thesis:

\section{Signed}

Candidate

Author 1

Author 2:

Author 3

Author 4

Author 5:

Author 6: 


\subsection{Inertia number calculation}

In order to neglect the effects of instrument inertia during LAOStress experiments, the ratio of instrument torque, $T_{i}$, and sample torque, $T_{s}$, has to be small. The ratio of instrument and sample torque is represented by the inertia number, $I_{n}$. If $I_{n} \leq 0.05$ the experimental results are acceptable, and the instrument inertia can be neglected.

The sample torque and instrument torque can be expressed as:

$$
\begin{aligned}
T_{S} & \sim \frac{2 \sigma_{0} \pi R^{3}}{3} \\
T_{i} & \sim I \omega^{2} \gamma_{0} \phi
\end{aligned}
$$

The inertia number is the ratio of instrument torque and sample torque $\left(I_{n}=T_{i} / T_{s}\right)$ can be calculated as:

$$
I_{n}=\frac{3 I \omega^{2} \phi \gamma_{0}}{2 \pi \sigma_{0} R^{3}}
$$

where

$\sigma_{0}-$ Stress amplitude $(\mathrm{Pa})$

$R$ - Radius of the cone and plate geometry (m)

$I-$ Instrument inertia $\left(\mathrm{Nm} \mathrm{s}^{2}\right)$

$\omega$ - Frequency $\left(\mathrm{rad} \mathrm{s}^{-1}\right)$

$\gamma_{0}-$ Strain amplitude (-)

$\phi$ - Cone angle (rad)

$R=20 \mathrm{~mm}=0.02 \mathrm{~m}$

$I=18.1983 \mu \mathrm{Nm} \mathrm{s}^{2}=1.8918^{*} 10^{-5} \mathrm{Nm} \mathrm{s}^{2}$

$\omega=1 \mathrm{~Hz}=6.283185 \mathrm{rad} \mathrm{s}^{-1}$

$\phi=2.01111^{\circ}=0.0351 \mathrm{rad}$ 
Table 8.1 Calculation of inertia number $\left(\mathrm{I}_{\mathrm{n}}\right)$ for applied stress amplitudes of $100 \mathrm{~Pa}, 500 \mathrm{~Pa}$ and $1500 \mathrm{~Pa}$

\begin{tabular}{||c|c|c|c|c|c|c|c|}
\hline \hline$\sigma_{0}$ & $\gamma_{0}$ & $\gamma_{0}$ & $\mathrm{~T}_{\mathrm{i}}$ & $\mathrm{T}_{\mathrm{s}}$ & $\mathrm{I}_{\mathrm{n}}\left(\mathrm{T}_{\mathrm{i}} / \mathrm{T}_{\mathrm{s}}\right)$ & $\begin{array}{c}\mathrm{I}_{\mathrm{n}} \\
\text { (formula) }\end{array}$ & $\mathrm{T}$ (total) \\
\hline $\mathrm{Pa}$ & $\%$ & - & $\mathrm{Nm}$ & $\mathrm{Nm}$ & - & - & $\mathrm{Nm}$ \\
\hline 100 & 6.81 & 0.0681 & $1.78526 \mathrm{E}-06$ & 0.001676 & 0.001066 & 0.001066 & 0.001677 \\
\hline 500 & 23.74 & 0.2374 & $6.22 \mathrm{E}-06$ & 0.008378 & 0.000743 & 0.000743 & 0.008384 \\
\hline 1500 & 53.11 & 0.5311 & $1.3923 \mathrm{E}-05$ & 0.025133 & 0.000554 & 0.000554 & 0.025147 \\
\hline
\end{tabular}

${ }^{*}$ Corrected stress was calculated as $\sigma_{0, \text { corr }}=3\left(T_{s}-T_{i}\right) / 2 \pi R^{3}$

Inertia number met the criteria of $I_{n} \leq 0.05$ for all stress values. 


\subsection{Examples of Gel Point measurements}

\subsubsection{Gel Point measured under the application of Small Amplitude Oscillatory Shear (SAOS)}

At the Gel Point (GP) the storage and loss moduli scale as power law in frequency, which allows us to identify the frequency independent GP. In the case of a viscoelastic liquid (VEL) (i.e. blood prior to GP), as the material is subjected to sinusoidal stress waveforms at different frequencies, the resulting response will be frequency dependent in such a way, that the lowest frequency will be on the top and highest on the bottom. As the material passes through its GP, and transitions into a viscoelastic solid (VES) (i.e. blood after GP) the order of the frequencies flip over, so that highest frequency will be on the top and lowest frequency in the bottom.

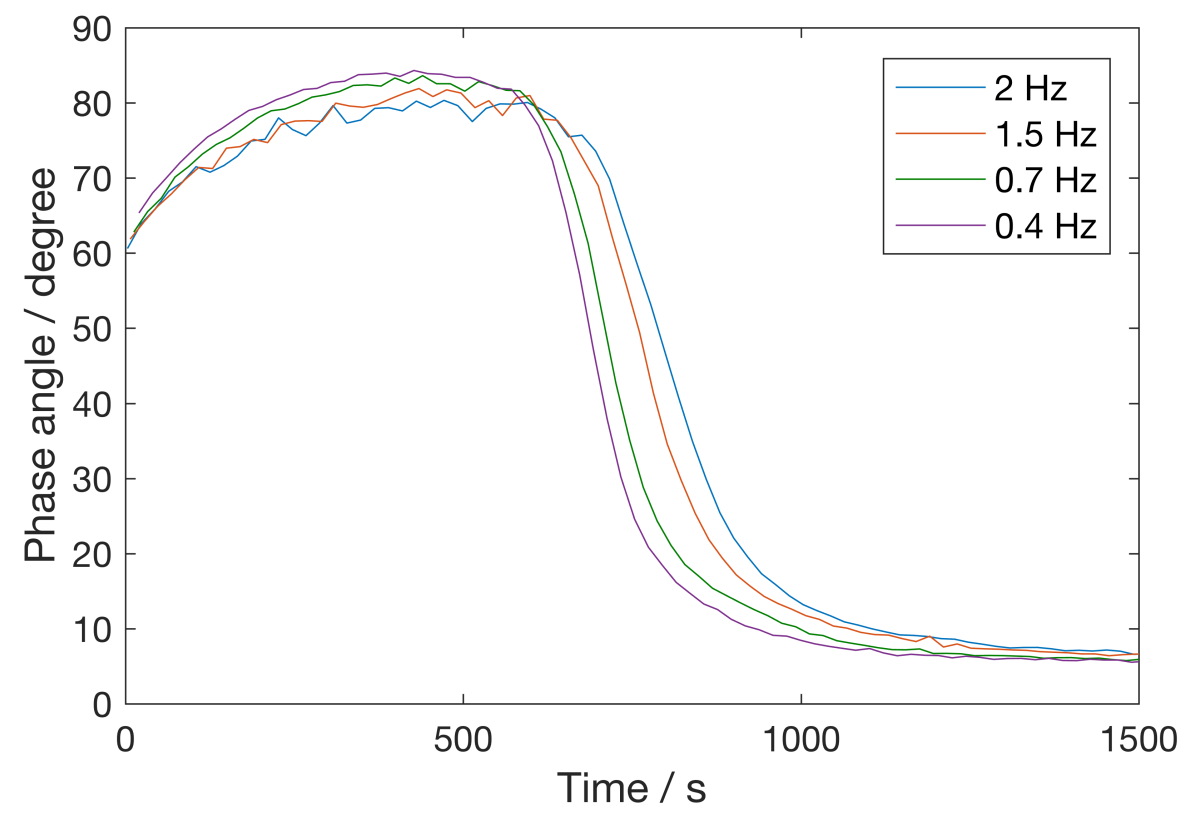

Figure 8.1 Material's response in terms of phase angle as a function of time in Gel Point measurement under the application of SAOS

\subsubsection{Gel Point measured under the application of Controlled Stress Parallel Superposition (CSPS)}

The GP under Controlled Stress Parallel Superposition (CSPS) is identified similar to that in SAOS. Once the material is passed its Gel Point, the unidirectional flow shear 
rate, $\dot{\gamma}_{s}$, becomes negligible, which suggests that the value of $G_{\|}^{\prime}, G_{\|}^{\prime \prime}$ and $\delta_{\|}$are approximately the equal to their corresponding measures in SAOS (Badiei et al. 2015).

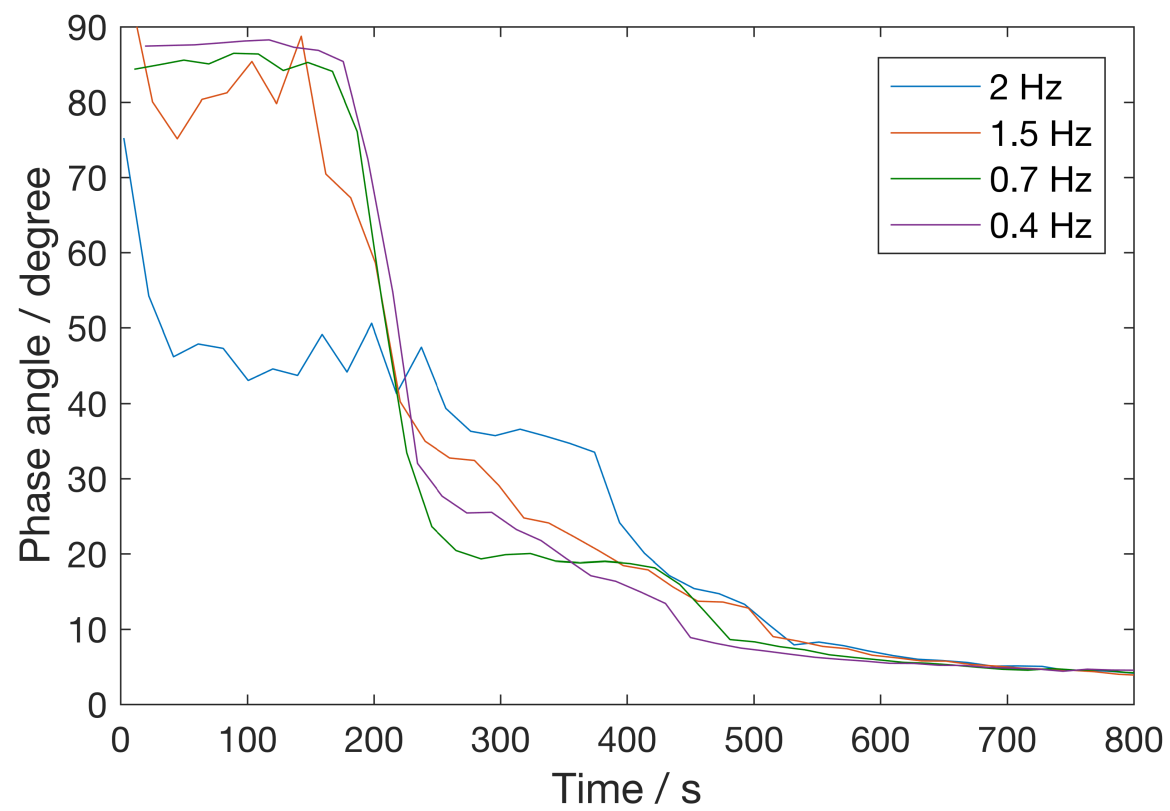

Figure 8.2 Material's response in terms of phase angle as a function of time in Gel Point measurement under the application of CSPS

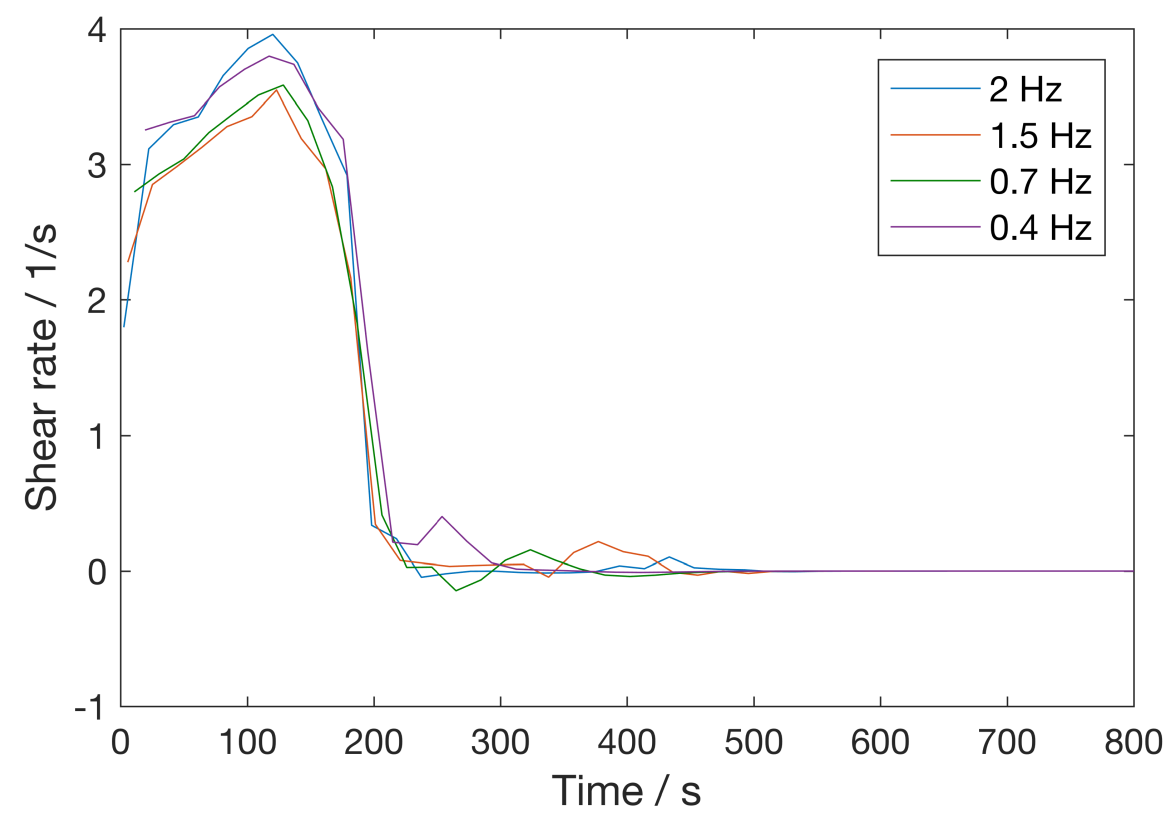

Figure 8.3 Material's response in terms of shear rate as a function of time in Gel Point measurement under the application of CSPS 


\subsection{Nomenclature}

Symbol

Definition

Unit

$\alpha$

$\alpha$

Stress relaxation component

Cone angle

A

Area

$\mathrm{m}^{2}$

$a_{1}$

Amplitude of fundamental frequency

$a_{k}$

Higher harmonic amplitudes

$\beta$

Small cone angle

Geometry factor

$c_{i, j}$

$c_{n}$

Mathematical constant

D

$n$th order Chebyshev compliance coefficient

$\mathrm{Pa}^{-1}$

Gap between rheometer plates

$\mu \mathrm{m}$

$D_{e}$

Embedding space

$d_{f}$

Fractal dimension

$\delta$

Phase angle

$\delta_{\|}$

$\delta_{D}$

Parallel superposition phase angle

Dirac delta

$E_{d}$

Dissipated energy

$\mathrm{J}$

$e_{n}$

Elastic Chebyshev coefficient

$\eta$

Viscosity

Pas

$\eta_{0}$

Zero shear viscosity

Pas

$\eta_{M}^{\prime}$

Minimum-rate dynamic viscosity

Pas

$\eta_{L}^{\prime}$

Large-rate dynamic viscosity

Pas

F

Force

$\mathrm{N}$

Strain factor

$\mathrm{N}$

$f_{i}$

$i$ th value of frequency of oscillation

$\mathrm{Hz}$

$f_{n}$

$n$th order Chebyshev fluidity coefficient

$\mathrm{Pas}^{-1}$

$F_{S}$

Sampling frequency

$F_{\sigma}$

Stress factor

$\mathrm{N}$

G

Modulus

$\mathrm{Pa}$

$G^{\prime}$

Storage modulus

$\mathrm{Pa}$ 


\begin{tabular}{|c|c|c|}
\hline$G^{\prime \prime}$ & Loss modulus & $\mathrm{Pa}$ \\
\hline$G^{*}$ & Complex modulus & $\mathrm{Pa}$ \\
\hline$\left|G_{\|}^{*}\right|$ & Parallel complex modulus & $\mathrm{Pa}$ \\
\hline$G_{\|}^{\prime}$ & Parallel storage modulus & $\mathrm{Pa}$ \\
\hline$G_{\|}^{\prime \prime}$ & Parallel loss modulus & $\mathrm{Pa}$ \\
\hline$G_{e}$ & Equilibrium modulus & $\mathrm{Pa}$ \\
\hline$G_{K}^{\prime}$ & Nonlinear tangent modulus & $\mathrm{Pa}$ \\
\hline$G_{L}^{\prime}$ & Large strain modulus & $\mathrm{Pa}$ \\
\hline$G_{M}^{\prime}$ & Minimum strain modulus & $\mathrm{Pa}$ \\
\hline$G_{\max }^{*}$ & Maximum complex modulus & $\mathrm{Pa}$ \\
\hline$G_{n}^{\prime}$ & $\begin{array}{l}n \text {th harmonic Fourier coefficient storage } \\
\text { modulus }\end{array}$ & $\mathrm{Pa}$ \\
\hline$G_{n}^{\prime \prime}$ & $\begin{array}{l}n \text {th harmonic Fourier coefficient loss } \\
\text { modulus }\end{array}$ & $\mathrm{Pa}$ \\
\hline$G(t)$ & Relaxation modulus & $\mathrm{Pa}$ \\
\hline$\gamma$ & Shear strain & - \\
\hline$\dot{\gamma}$ & Shear rate & $\mathrm{s}^{-1}$ \\
\hline$\dot{\gamma}_{s}$ & Unidirectional flow shear rate & $1 / \mathrm{s}$ \\
\hline$\gamma_{0}$ & Strain amplitude & - \\
\hline$\gamma^{e}$ & Elastic strain component & - \\
\hline$\gamma^{v}$ & Viscous strain component & - \\
\hline$h$ & Gap between rheometer plates & $\mu \mathrm{m}$ \\
\hline$H$ & Height & $\mathrm{m}$ \\
\hline$I$ & Instrument inertia & $\mathrm{Nm} \mathrm{s}^{2}$ \\
\hline$I_{n}$ & Inertia number & - \\
\hline$I_{n / 1}$ & Normalised intensity & - \\
\hline$J^{\prime}$ & Linear elastic storage compliance & $\mathrm{Pa}^{-1}$ \\
\hline$J^{\prime \prime}$ & Linear elastic loss compliance & $\mathrm{Pa}^{-1}$ \\
\hline$J^{*}$ & Complex compliance & $\mathrm{Pa}^{-1}$ \\
\hline$J_{e}$ & Equilibrium creep compliance & $\mathrm{Pa}$ \\
\hline$J_{K}^{\prime}$ & Nonlinear tangent compliance & $\mathrm{Pa}^{-1}$ \\
\hline$J_{L}^{\prime}$ & Large stress elastic compliance & $\mathrm{Pa}^{-1}$ \\
\hline
\end{tabular}




\begin{tabular}{|c|c|c|}
\hline$J_{M}^{\prime}$ & Zero stress elastic compliance & $\mathrm{Pa}^{-1}$ \\
\hline$J_{n}^{\prime}$ & $n$th order storage compliance & $\mathrm{Pa}^{-1}$ \\
\hline$J_{n}^{\prime \prime}$ & $n$th order loss compliance & $\mathrm{Pa}^{-1}$ \\
\hline$J(t)$ & Creep compliance & $\mathrm{Pa}$ \\
\hline$K^{\prime}$ & Differential (tangent) storage modulus & $\mathrm{Pa}$ \\
\hline$K^{\prime \prime}$ & Differential (tangent) loss modulus & $\mathrm{Pa}$ \\
\hline$K^{*}$ & Differential (tangent) complex modulus & $\mathrm{Pa}$ \\
\hline$l$ & Wavelength & $\mathrm{nm}$ \\
\hline$\lambda$ & Characteristic relaxation rate & $\mathrm{s}^{-1}$ \\
\hline$\lambda_{m u}$ & Mutation time & $\mathrm{s}$ \\
\hline$M$ & Torque & $\mathrm{Nm}$ \\
\hline$N$ & Number of cycles & - \\
\hline$N_{f f t}$ & Number of Fourier spectra points & - \\
\hline$N_{m u}$ & Mutation number & - \\
\hline$p$ & Chemical conversion & - \\
\hline$p_{c}$ & Critical chemical conversion & - \\
\hline$Q$ & Nonlinear coefficient & - \\
\hline$R$ & Nonlinear compliances ratio & - \\
\hline$R$ & Radius & $\mathrm{m}$ \\
\hline$\rho$ & Density & $\mathrm{kg}\left(\mathrm{m}^{3}\right)^{-1}$ \\
\hline$S$ & Strength of the network at GP & - \\
\hline$S$ & Strain stiffening ratio & - \\
\hline$S(\omega)$ & Frequency spectrum & - \\
\hline$s(t)$ & Time signal & - \\
\hline$\sigma$ & Shear stress & $\mathrm{Pa}$ \\
\hline$\sigma_{0}$ & Stress amplitude & $\mathrm{Pa}$ \\
\hline$\sigma^{\prime}$ & Elastic stress component & $\mathrm{Pa}$ \\
\hline$\sigma^{\prime \prime}$ & Viscous stress component & $\mathrm{Pa}$ \\
\hline$\sigma_{f}$ & Fracture stress & $\mathrm{Pa}$ \\
\hline$\sigma_{s}$ & Unidirectional flow component of stress & $\mathrm{Pa}$ \\
\hline$t$ & Time & s \\
\hline$T$ & Torque & $\mathrm{Nm}$ \\
\hline$T$ & Shear thickening ratio & - \\
\hline
\end{tabular}




\begin{tabular}{|c|c|c|}
\hline $\tan \delta$ & Loss tangent & $\circ$ \\
\hline$t_{a q}$ & Acquisition time & $\mathrm{s}$ \\
\hline$t_{c}$ & Conditioning time & $\mathrm{s}$ \\
\hline$t_{d w}$ & Dwell time & $\mathrm{s}$ \\
\hline$t_{\text {exp }}$ & Experiment time & $\mathrm{s}$ \\
\hline \multirow[t]{2}{*}{$T_{n}(x)$} & $n$th order Chebyshev polynomial of the first & - \\
\hline & kind & \\
\hline$\tau$ & Relaxation time & s \\
\hline$\tau_{J}$ & Retardation time & $\mathrm{S}$ \\
\hline$v$ & Velocity & $\mathrm{m} \mathrm{s}^{-1}$ \\
\hline$v$ & Fourier frequency & $\operatorname{rad~s}^{-1}$ \\
\hline$v_{n}$ & Viscous Chebyshev coefficient & - \\
\hline$\delta \theta$ & Distortion angle & $\circ$ \\
\hline$\phi$ & Fluidity & $\operatorname{Pas}^{-1}$ \\
\hline$\phi_{n}^{\prime}$ & $n$th order storage fluidity & $\mathrm{Pas}^{-1}$ \\
\hline$\phi_{n}^{\prime \prime}$ & $n$th order loss fluidity & $\mathrm{Pas}^{-1}$ \\
\hline$\omega$ & Angular frequency & $\operatorname{rad~s}^{-1}$ \\
\hline$\phi_{L}^{\prime}$ & Large stress fluidity & $\mathrm{Pas}^{-1}$ \\
\hline$\phi_{M}^{\prime}$ & Zero stress fluidity & $\mathrm{Pas}^{-1}$ \\
\hline$\omega$ & Angular velocity & $\operatorname{rad~s}^{-1}$ \\
\hline$\omega_{*}$ & Inertio-elastic ringing frequency & $\operatorname{rad~s}^{-1}$ \\
\hline$\Delta$ & Logarithmic decrement & - \\
\hline
\end{tabular}




\section{References}

Ariens, R.A.S., 2009. Denser matters. Blood, 114(19), pp.3978-3979.

Atalik, K. \& Keunings, R., 2004. On the occurrence of even harmonics in the shear stress response of viscoelastic fluids in large amplitude oscillatory shear. Journal of Non-Newtonian Fluid Mechanics, 122, pp.107-116.

Aujesky, D. et al., 2005. Derivation and Validation of a Prognostic Model for Pulmonary Embolism. Am J Respir Crit Care Med, 172, pp.1041-1046.

Austin, S.K., 2017. Haemostasis. Medicine, 45(4), pp.204-208.

Badiei, N. et al., 2015. Effects of unidirectional flow shear stresses on the formation, fractal microstructure and rigidity of incipient whole blood clots and fibrin gels. Clinical Hemorheology and Microcirculation, 60(4), pp.451-464. Available at: http://www.medra.org/servlet/aliasResolver?alias=iospress\&doi=10.3233/CH151924.

Bale, M.D. \& Ferry, J.D., 1988. Strain enhancement of elastic modulus in fine fibrin clots. Thromb Res., 52(6), pp.565-72.

Baravian, C., Benbelkacem, G. \& Caton, F., 2007. Unsteady rheometry: Can we characterize weak gels with a controlled stress rheometer? Rheologica Acta, 46(5), pp.577-581.

Barnes, G.D. et al., 2014. Venous thromboembolism: Diagnosis, treatment and the prevention of long-term complications. Reviews in Vascular Medicine, 2, pp.136-142.

Barnes, H.A., 2000. A handbook of elementary rheology, Institute of Non-Newtonian Fluid Mechanics, Univeristy of Wales.

Bayer HealthCare, 2013. From prevention to treatment deep vein thrombosis and pulmonary embolism,

Bird, R.B., Armstrong, R.C. \& Hassager, O., 1977. Dynamics of Polymeric Liquids, Volume 1: Fluid Mechanics,

Bodnár, T., Sequeira, A. \& Prosi, M., 2011. On the shear-thinning and viscoelastic effects of blood flow under various flow rates. Applied Mathematics and Computation, 217(11), pp.5055-5067. Available at: http://dx.doi.org/10.1016/j.amc.2010.07.054.

Broedersz, C.P. et al., 2010. Measurement of nonlinear rheology of cross-linked biopolymer gels. Soft Matter, 6, pp.4120-4127. 
Brown, A.E.X. et al., 2009. Multiscale Mechanics of Fibrin Polymer : Gel Stretching with Protein Unfolding and Loss of Water. Science, 325, pp.741-744.

Campbell, R.A. et al., 2008. Cellular Procoagulant Activity Dictates Clot Structure and Stability as a Function of Distance From the Cell Surface. Arterioscler Thromb Vasc Biol., 28(12), pp.2247-2254.

Campbell, R.A. et al., 2010. Flow profoundly influences fibrin network structure: Implications for fibrin formation and clot stability in haemostasis. Thromb Haemost., 104(6), pp.1281-1284.

Chambon, F. \& Winter, H.H., 1987. Linear Viscoelasticity at the Gel Point of a Crosslinking PDMS with Imbalanced Stoichiometry. Journal of Rheology, 31, p.683.

Chambon, F. \& Winter, H.H., 1985. Stopping of crosslinking reaction in a PDMS polymer at the gel point. Polymer Bulletin, 13(6), pp.499-503.

Chambon, F.H., 1986. Linear viscoelasticity of crosslinking polymers at the gel point. University of Massachusetts. Available at:

https://scholarworks.umass.edu/cgi/viewcontent.cgi?referer=https://www.googl e.co.uk/\&httpsredir=1\&article $=1708 \&$ context $=$ dissertations_ 1 .

Charm, S.E. \& Kurland, G.S., 1967. Static Method for determining Blood Yield Stress. Nature, 216, pp.1121-1123.

Chernysh, I.N. et al., 2012. Fibrin Clots Are Equilibrium Polymers That Can Be Remodeled Without Proteolytic Digestion. Scientific reports, 2(879), pp.1-6.

Cho, K.S. et al., 2005. A geometrical interpretation of large amplitude oscillatory shear response. Journal of Rheology, 49(3), pp.747-758.

Clemetson, K.J., 2012. Platelets and primary haemostasis. Thrombosis Research, 129(3), pp.220-224. Available at: http://dx.doi.org/10.1016/j.thromres.2011.11.036.

Colace, T. V, Muthard, R.W. \& Diamond, S.L., 2012. Thrombus Growth and Embolism on Tissue Factor-Bearing Collagen Surfaces Under Flow: Role of Thrombin With and Without Fibrin. Arterioscler Thromb Vasc Biol., 32, pp.1466-1476.

Collen, D. \& Lijnen, H.R., 1991. Basic and clinical aspects of fibrinolysis and thrombolysis. Blood, 78(12), pp.3114-3124. Available at: http://www.researchgate.net/publication/21412268.

Collet, J.P. et al., 2002. A Structural and Dynamic Investigation of the Facilitating 
Effect of Glycoprotein IIb/IIIa Inhibitors in Dissolving Platelet-Rich Clots. Circulation research, 90(4), pp.428-435.

Corral, J., Roldan, V. \& Vicente, V., 2010. Deep venous thrombosis or pulmonary embolism and factor V Leiden: enigma or paradox. Haematologica, 95(6), pp.863-866.

Costello, B., The AR-G2 Magnetic Bearing Rheometer., pp.1-6. Available at: http://www.tainstruments.com/pdf/literature/RH085_AR_G2_performance.pdf [Accessed January 20, 2018].

Curtis, D.J. et al., 2013. A study of microstructural templating in fibrin-thrombin gel networks by spectral and viscoelastic analysis. Soft Matter, 9, pp.4883-4889. Available at: http://xlink.rsc.org/?DOI=c3sm50263e.

Curtis, D.J. et al., 2015. Assessment of the stress relaxation characteristics of critical gels formed under unidirectional shear flow by controlled stress parallel superposition rheometry. Journal of Non-Newtonian Fluid Mechanics, 222, pp.227-233. Available at: http://dx.doi.org/10.1016/j.jnnfm.2014.12.004.

Curtis, D.J. et al., 2011. Rheometrical and molecular dynamics simulation studies of incipient clot formation in fibrin-thrombin gels: An activation limited aggregation approach. Journal of Non-Newtonian Fluid Mechanics, 166, pp.932-938. Available at: http://dx.doi.org/10.1016/j.jnnfm.2011.04.016.

Dimitriou, C.J., Ewoldt, R.H. \& McKinley, G.H., 2013. Describing and prescribing the constitutive response of yield stress fluids using large amplitude oscillatory shear stress (LAOStress). Journal of Rheology, 57, pp.27-70.

Dintenfass, L., 1962. Thixotropy of blood and proneness to thrombus formation. Journal of the American Heart Research, XI, pp.233-239.

van Dusschoten, D. \& Wilhelm, M., 2001. Increased torque transducer sensitivity via oversampling. Rheologica Acta, 40(4), pp.395-399.

Evans, P.A. et al., 2010. Gel point and fractal microstructure of incipient blood clots are significant new markers of hemostasis for healthy and anticoagulated blood. Blood, 116(17), pp.3341-3347.

Evans, P.A. et al., 2008. Rheometry and associated techniques for blood coagulation studies. Medical Engineering and Physics, 30, pp.671-679.

Ewoldt, R.H. et al., 2010. Large amplitude oscillatory shear of pseudoplastic and elastoviscoplastic materials. Rheologica Acta, 49(2), pp.191-212.

Ewoldt, R.H., 2009. Nonlinear viscoelastic materials: bioinspired applications and 
new characterization measures by. Massachusetts Institute of Technology.

Ewoldt, R.H. \& Bharadwaj, N.A., 2013. Low-dimensional intrinsic material functions for nonlinear viscoelasticity. Rheologica Acta, 52(3), pp.201-219.

Ewoldt, R.H., Hosoi, A.E. \& McKinley, G.H., 2008. New measures for characterizing nonlinear viscoelasticity in large amplitude oscillatory shear. Journal of Rheology, 52, pp.1427-1458.

Ewoldt, R.H., Johnston, M.T. \& Caretta, L.M., 2015. Experimental Challenges of Shear Rheology: How to Avoid Bad Data. In S. Spagnolie, ed. Complex Fluids in Biological Systems. Biological and Medical Physics, Biomedical Engineering. New York, NY: Springer, pp. 207-241. Available at: http://link.springer.com/10.1007/978-1-4939-2065-5_6.

Ewoldt, R.H. \& McKinley, G.H., 2007. Creep Ringing in Rheometry or How to Deal with Oft-discarded Data in Step Stress Tests! Rheology Bulletin, 76(1), pp.4-25.

Ewoldt, R.H., Winegard, T.M. \& Fudge, D.S., 2011. Non-linear viscoelasticity of hagfish slime. International Journal of Non-Linear Mechanics, 46, pp.627-636. Available at: http://dx.doi.org/10.1016/j.ijnonlinmec.2010.10.003.

Feghhi, S. \& Sniadecki, N.J., 2011. Mechanobiology of Platelets: Techniques to Study the Role of Fluid Flow and Platelet Retraction Forces at the Micro- and Nano-Scale. Int J Mol Sci., 12(12), pp.9009-9030.

Ferry, J.D., 1952. The mechanism of polymerization of fibrinogen. Chemistry, 38, pp.566-569.

Ferry, J.D., 1980. Viscoelastic Properties of Polymers, John Wiley \& Sons.

Flamm, M.H. \& Diamond, S.L., 2012. Multiscale Systems Biology and Physics of Thrombosis under Flow. Ann Biomed Eng., 40(11), pp.2355-2364.

Flory, P.J., 1953. Principles of Polymer Chemistry, Cornell University Press. Franchini, M., 2005. Heparin-induced thrombocytopenia: an update. Thrombosis Journal, 3, p.14. Available at: http://thrombosisjournal.biomedcentral.com/articles/10.1186/1477-9560-3-14. Le Gal, G. et al., 2006. Prediction of Pulmonary Embolism in the Emergency Department: The Revised Geneva Score. Ann Intern Med., 144(3), p.165-171. de Gennes, P.-G., 1979. Scaling Concepts in Polymer Physics, Cornell University Press.

Gersh, K.C., Edmondson, K.E. \& Wiesel, J.W., 2010. Flow rate and fibrin fiber alignment. J Thromb Haemost., 8(12), pp.2826-2828. 
Gersh, K.C., Nagaswami, C. \& Weisel, J.W., 2009. Fibrin network structure and clot mechanical properties are altered by incorporation of erythrocytes. Thromb Haemost., 102(6), pp.1169-1175.

Giacomin, A.J. \& Dealy, J.M., 1993. Large-Amplitude Oscillatory Shear. In A. A. Collyer, ed. Techniques in Rheological Measurement. SpringerScience+Business Media, B. V., pp. 99-121.

Gibson, N.S. et al., 2008. Further validation and simplification of the Wells clinical decision rule in pulmonary embolism. Thromb Haemost., 99(1), pp.229-234.

Gijsen, F.J. et al., 1999. The influence of the non-Newtonian properties of blood on the flow in large arteries: unsteady flow in a 90 degrees curved tube. Journal of Biomechanics, 32(7), pp.705-713.

Gruber, M.P. \& Bull, T.M., 2012. Pulmonary Embolism. In Clinical Respiratory Medicine (Fourth Edition). pp. 690-709.

Hawkins, K. et al., 2010. The development of rheometry for strain-sensitive gelling systems and its application in a study of fibrin-thrombin gel formation. Rheologica Acta, 49(9), pp.891-900.

Hendriksen, J.M.T. et al., 2015. Diagnostic prediction models for suspected pulmonary embolism: Systematic review and independent external validation in primary care. $B M J, 351$, pp.1-9.

Henning, S. \& Adhikari, R., 2017. Scanning Electron Microscopy, ESEM, and X-ray Microanalysis. In Microscopy Methods in Nanomaterials Characterization.

Elsevier Inc., pp. 1-30. Available at: https://scholarworks.umass.edu/cgi/viewcontent.cgi?referer=https://www.googl e.co.uk/\&httpsredir=1\&article $=1708 \&$ context=dissertations_1.

Hilliou, L. et al., 2004. Increasing the Force Torque Transducer Sensitivity of an RPA 2000 by a Factor 5 - 10 via Advanced Data Acquisition. Rubber Chemistry and Technology, 77(1), pp.192-200.

Hitosugi, M., Niwa, M. \& Takatsu, A., 2001. Changes in Blood Viscosity by Heparin and Argatroban. Thrombosis research, 104, pp.371-374.

Hoffman, M., 2008. Alterations of fibrinogen structure in human disease. Cardiovasc Hematol Agents Med Chem., 6(3), pp.206-211.

Hudson, N.E. et al., 2010. Stiffening of Individual Fibrin Fibers Equitably Distributes Strain and Strengthens Networks. Biophysical Journal, 98, pp.16321640. 
Hyun, K. et al., 2011. A review of nonlinear oscillatory shear tests : Analysis and application of large amplitude oscillatory shear ( LAOS ). Progress in Polymer Science, 36, pp.1697-1753. Available at: http://dx.doi.org/10.1016/j.progpolymsci.2011.02.002.

Hyun, K. et al., 2007. Fourier-transform rheology under medium amplitude oscillatory shear for linear and branched polymer melts. Journal of Rheology, 51, pp.1319-1342.

Hyun, K. et al., 2002. Large amplitude oscillatory shear as a way to classify the complex fluids. J. Non-Newtonian Fluid Mech., 107, pp.51-65.

Hyun, K. \& Wilhelm, M., 2009. Establishing a New Mechanical Nonlinear Coefficient Q from FT-Rheology: First Investigation of Entangled Linear and Comb Polymer Model Systems. Macromolecules, 42(1), pp.411-422.

Janmey, P.A. et al., 2007. Negative normal stress in semiflexible biopolymer gels. Nat Mater., 6(1), pp.48-51.

Janmey, P.A., Amis, E.J. \& Ferry, J.D., 1983. Rheology of Fibrin Clots. VI. Stress Relaxation, Creep, and Differential Dynamic Modulus of Fine Clots in Large Shearing Deformations. Journal of Rheology, 27(135).

Janmey, P.A., Winer, J.P. \& Weisel, J.W., 2009. Fibrin gels and their clinical and bioengineering applications. Journal of The Royal Society Interface, 6(30), pp.1-10. Available at: http://rsif.royalsocietypublishing.org/cgi/doi/10.1098/rsif.2008.0327.

Jansen, K.A. et al., 2013. Cells Actively Stiffen Fibrin Networks by Generating Contractile Stress. Biophysical Journal, 105(10), pp.2240-2251. Available at: http://dx.doi.org/10.1016/j.bpj.2013.10.008.

Jawerth, L., Muenster, S. \& Weitz, D.A., 2013. The Mechanical Mechanism of Platelet Induced Clot Stiffening. Biophys J., 104(2), p.150a. Available at: http://dx.doi.org/10.1016/j.bpj.2012.11.851.

Jen, C.J. \& McIntire, L. V, 1982. The structural properties and contractile force of a clot. Cell Motil., 2(5), pp.445-455.

Jha, A.K. et al., 2013. The global burden of unsafe medical care : analytic modelling of observational studies. BMJ Qual Saf, 22, pp.809-815.

Kallus, S. et al., 2001. Characterization of polymer dispersions by Fourier transform rheology. Rheologica Acta, 40(6), pp.552-559.

Kang, H. et al., 2009. Nonlinear Elasticity of Stiff Filament Networks: Strain 
Stiffening, Negative Normal Stress, and Filament Alignment in Fibrin Gels. $J$. Phys. Chem. B, 113, pp.3799-3805.

Kearon, C. et al., 2008. Influence of thrombophilia on risk of recurrent venous thromboembolism while on warfarin: results from a randomized trial. Blood, 112(12), pp.4432-4436.

van Kempen, T.H.S., Donders, W.P. \& van de Vosse, F.N., 2016. A constitutive model for developing blood clots with various compositions and their nonlinear viscoelastic behavior. Biomech Model Mechanobiol, 15, pp.279-291.

van Kempen, T.H.S., Peters, G.W.M. \& van de Vosse, F.N., 2015. A constitutive model for the time-dependent, nonlinear stress response of fibrin networks. Biomech Model Mechanobiol, 14, pp.995-1006.

Keshavarz, B. et al., 2017. Nonlinear viscoelasticity and generalized failure criterion for biopolymer gels. ACS Macro Lett., 6(7), pp.663-667.

Kim, O. V et al., 2014. Biomaterials Structural basis for the nonlinear mechanics of fi brin networks under compression. Biomaterials, 35(25), pp.6739-6749. Available at: http://dx.doi.org/10.1016/j.biomaterials.2014.04.056.

Kim, S. et al., 2000. A new method for blood viscosity measurement. J. NonNewtonian Fluid Mech., 94, pp.47-56.

Klok, F.A. et al., 2008. Simplification of the revised Geneva score for assessing clinical probability of pulmonary embolism. Arch Intern Med., 168(19), p.2131-6.

Konstantinides, S. \& Goldhaber, S.Z., 2012. Pulmonary embolism: risk assessment and management embolism. European Heart Journal, 33, pp.3014-3022.

Kroll, M.H. et al., 1996. Platelets and shear stress. Blood, 88(5), pp.1525-1541.

Lam, W.A. et al., 2011. Mechanics and contraction dynamics of single platelets and implications for clot stiffening. Nat Mater., 10(1), pp.61-66.

Läuger, J. \& Stettin, H., 2010. Differences between stress and strain control in the non-linear behavior of complex fluids. Rheol Acta, 49, pp.909-930.

Lawrence, M.J. et al., 2015. A new biomarker quantifies differences in clot microstructure in patients with venous thromboembolism. Br J Haematol., 168(4), pp.571-575.

Lawrence, M.J. et al., 2014. A new structural biomarker that quanti fi es and predicts changes in clot strength and quality in a model of progressive haemodilution. Thrombosis research, 134, pp.488-494. 
Lenk, R.S., 1978. Polymer Rheology, Applied Science Publishers.

Li, W. et al., 2016. Fibrin Fiber Stiffness Is Strongly Affected by Fiber Diameter, but Not by Fibrinogen Glycation. Biophys J., 110(6), pp.1400-1410. Available at: http://dx.doi.org/10.1016/j.bpj.2016.02.021.

Litvinov, R.I. \& Weisel, J.W., 2017. Fibrin mechanical properties and their structural origins. Matrix Biology, 60-61, pp.110-123. Available at: http://dx.doi.org/10.1016/j.matbio.2016.08.003.

Liu, W. et al., 2006. Fibrin Fibers Have Extraordinary Extensibility and Elasticity. Science, 313, p.634.

Liu, W. et al., 2010. The mechanical properties of single fibrin fibers. J Thromb Haemost., 8(5), pp.1030-1036.

Loglio, G. et al., 2004. Oscillation of interfacial properties in liquid systems: assessment of harmonic distortion. Physical Chemistry Chemical Physics, 6(7), pp.1375-1379. Available at: http://xlink.rsc.org/?DOI=b314592c.

Martinez, M.R. et al., 2014. Enhanced lysis and accelerated establishment of viscoelastic properties of fibrin clots are associated with pulmonary embolism. Am J Physiol Lung Cell Mol Physiol, 306, pp.L397-L404.

May, R. \& Thurner, J., 1957. The cause of the predominantly sinistral occurrence of thrombosis of the pelvic veins. Angiology, 8(5), pp.419-427.

Mezger, T.G., 2015. Applied Rheology 2nd ed., Anton Paar GmbH.

Miller, R. \& Liggieri, L., 2009. Interfacial Rheology R. Miller \& L. Liggieri, eds., CRC Press.

de Moerloose, P. \& Neerman-Arbez, M., 2009. Congenital fibrinogen disorders. Semin Thromb Hemost., 35(4), pp.356-366.

Munster, S. et al., 2013. Structure and mechanics of fibrin clots formed under mechanical perturbation. J Thromb Haemost., 11, pp.557-560.

Münster, S. et al., 2013. Strain history dependence of the nonlinear stress response of fi brin and collagen networks. PNAS, 110(30), pp.12197-12202.

Muthukumar, M., 1985. Dynamics of polymeric fractals. The Journal of Chemical Physics, 83, p.3161.

Muthukumar, M. \& Winter, H.H., 1986. Fractal dimension of a crosslinking polymer at the gel point. Macromolecules, 19(4), pp.1284-1285.

National Assembly for Wales Health and Social Care Committee, 2012. One-day inquiry into venous thromboebmolism prevention in hospitalised patients in 
Wales,

Nhs.uk, 2016. Deep vein thrombosis. Available at:

https://www.nhs.uk/conditions/deep-vein-thrombosis-dvt/ [Accessed October 20, 2014].

NICE, 2012. Venous thromboembolic diseases: diagnosis, management and thrombophilia testing,

Ogston, C.M. \& Ogston, D., 1966. Plasma fibrinogen and plasminogen levels in health and in ischaemic heart disease. Journal of clinical pathology, 19(4), pp.352-356.

Oka, S., 1981. Cardiovascular Hemorheology, Cambridge: Cambridge University Press.

Piechocka, I.K. et al., 2010. Structural Hierarchy Governs Fibrin Gel Mechanics. Biophysical Journal, 98, pp.2281-2289. Available at: http://dx.doi.org/10.1016/j.bpj.2010.01.040.

Pirofsky, B., 1952. The determination of blood viscosity in man by a method based on Poiseuille's law. J. Clin. Invest., 32, pp.292-298.

Purohit, P.K. et al., 2011. Protein unfolding accounts for the unusual mechanical behavior of fibrin networks. Acta Biomaterialia, 7(6), pp.2374-2383. Available at: http://dx.doi.org/10.1016/j.actbio.2011.02.026.

Report to the Chief Medical Officer, 2007. Dept of Health,

Riha, P. et al., 1999. Elasticity and fracture strain of whole blood clots. Clin Hemorheol Microcirc., 21(1), pp.45-49.

Roberts, H.R. \& White, G.C., 2017. The biology of haemostasis and thrombosis. In Oxford Textbook of Medicine. Oxford University Press.

Rogers, S.A. \& Vlassopoulos, D., 2012. Frieze group analysis of asymmetric response to large-amplitude oscillatory shear. Journal of Rheology, 54(4), p.859.

van Rooy, M.J. et al., 2015. Platelet hyperactivity and fibrin clot structure in transient ischemic attack individuals in the presence of metabolic syndrome: A microscopy and thromboelastography® study. Cardiovascular Diabetology, 14(86), pp.1-13. Available at: http://dx.doi.org/10.1186/s12933-015-0249-5.

Ryan, E.A. et al., 1999. Structural Origins of Fibrin Clot Rheology. Biophysical Journal, 77(5), pp.2813-2826. Available at: http://linkinghub.elsevier.com/retrieve/pii/S0006349599771134. 
Savushkin, A. V, 2003. Clots of Blood Plasma. Journal of Engineering Physics and Thermophysics, 76(3), pp.645-647.

Scanlan, J.C. \& Winter, H.H., 1991. Composition dependence of the viscoelasticity of end-linked poly(dimethylsiloxane) at the gel point. Macromolecules, 24(1), pp.47-54.

Schellong, S.M. et al., 2003. Complete compression ultrasonography of the leg veins as a single test for the diagnosis of deep vein thrombosis. Thromb Haemost., 89(2), pp.228-234.

Semmrich, C., Larsen, R.J. \& Bausch, A.R., 2008. Nonlinear mechanics of entangled F-actin solutions. Soft Matter, 4, pp.1675-1680.

Shah, J. V \& Janmey, P.A., 1997. Strain hardening of fibrin gels and plasma clots. Rheol Acta, 36, pp.262-268.

Shakerian, F. \& Sadeghipour, P., 2018. Venous Thromboembolism. In Practical Cardiology. Elsevier Inc., pp. 499-523. Available at: http://dx.doi.org/10.1016/B978-0-323-51149-0.00029-8.

Sim, H.G., Ahn, K.H. \& Lee, S.J., 2003. Large amplitude oscillatory shear behavior of complex fluids investigated by a network model: A guideline for classification. J. Non-Newtonian Fluid Mech., 112, pp.237-250.

Sousa, P.C. et al., 2013. Shear viscosity and nonlinear behavior of whole blood under large amplitude oscillatory shear. Biorheology, 50, pp.269-282.

Stanford, S.N. et al., 2015. The changes in clot microstructure in patients with ischaemic stroke and the effects of therapeutic intervention: A prospective observational study. BMC Neurology, 15(1), pp.1-8. Available at: https://www.ncbi.nlm.nih.gov/pmc/articles/PMC4367933/pdf/12883_2015_Arti cle_289.pdf.

Storm, C. et al., 2005. Nonlinear elasticity in biological gels. Nature, 435, pp.191194.

Sun, W. et al., 2015. Large amplitude oscillatory shear studies on the strainstiffening behavior of gelatin gels. Chinese Journal of Polymer Science, 33(1), pp. $70-83$.

TA Instruments, 2014. ARES-G2 Rheometer Getting Started Guide,

Tassieri, M. et al., 2016. i-Rheo: Measuring the materials' linear viscoelastic properties "in a step " $</ i>$ ! Journal of Rheology, 60(4), pp.649-660. Available at: http://sor.scitation.org/doi/10.1122/1.4953443. 
Tassieri, M. et al., 2018. I-Rheo GT: Transforming from Time to Frequency Domain without Artifacts. Macromolecules, 51(14), pp.5055-5068.

Tassieri, M., 2016. Microrheology with Optical Tweezers: Principles and Applications, Pan Stanford Publishing Pte. Ltd.

Tee, T. \& Dealy, J.M., 1975. Nonlinear Viscoelasticity of Polymer Melts. Journal of Rheology, 19, p.595.

Thachil, J., 2012. Recurrent venous thromboembolism while on anticoagulant therapy. Blood Reviews, 26, pp.175-181. Available at: http://dx.doi.org/10.1016/j.blre.2012.04.002.

Thomas, B., 2014. A Rheological Biomarker for the Detection of Heparin Induced Thrombocytopenia. Swansea University.

Thurston, G.B., 1972. Viscoelasticity of human blood. Biophysical Journal, 12(4), pp.1205-1217.

Tripathi, M.M. et al., 2017. Clinical evaluation of whole blood prothrombin time (PT) and international normalized ratio (INR) using a Laser Speckle Rheology sensor. Scientific Reports, 7(1), pp.1-8. Available at: http://dx.doi.org/10.1038/s41598-017-08693-5.

Tung, C.M. \& Dynes, P.J., 1982. Relationship between viscoelastic properties and gelation in thermosetting systems. Journal of Applied Polymer Science, 27(2), pp.569-574.

Undas, A. et al., 2009. Altered fibrin clot structure/function in patients with idiopathic venous thromboembolism and in their relatives. Blood, 114(19), pp.4272-4278.

Undas, A. et al., 2008. Reduced clot permeability and susceptibility to lysis in patients with acute coronary syndrome: Effects of inflammation and oxidative stress. Atherosclerosis, 196, pp.551-557.

Undas, A. \& Ariens, R.A.S., 2011. Fibrin clot structure and function: a role in the pathophysiology of arterial and venous thromboembolic diseases. Arterioscler Thromb Vasc Biol., 31(12), pp.e88-99.

Varju, I. et al., 2011. Hindered dissolution of fibrin formed under mechanical stress. Journal of Thrombosis and Haemostasis, 9(5), pp.979-986.

Veklich, Y. et al., 1998. Structural studies of fibrinolysis by electron microscopy. Blood, 92(12), pp.4721-4729.

Vinson, D.R. et al., 2016. Risk stratifying emergency department patients with acute 
pulmonary embolism: Does the simplified Pulmonary Embolism Severity Index perform as well as the original? Thrombosis Research, 148, pp.1-8. Available at: http://dx.doi.org/10.1016/j.thromres.2016.09.023.

Weisel, J.W., 2007. Structure of fibrin: Impact on clot stability. Journal of Thrombosis and Haemostasis, 5(Suppl. 1), pp.116-124.

Weisel, J.W. \& Litvinov, R.I., 2013a. Adaptation of fi brous biopolymers to recurring increasing strains. PNAS, 110(30), pp.12164-12165.

Weisel, J.W. \& Litvinov, R.I., 2013b. Mechanisms of fibrin polymerization and clinical implications. Blood, 121(10), pp.1712-1720.

Weisel, J.W. \& Litvinov, R.I., 2014. Mechanisms of Fibrinolysis and Basic Principles of Management. In Hemostasis and Thrombosis: Practical Guidelines in Clinical Management. John Wiley \& Sons Ltd., pp. 169-185.

Wells, P.S. et al., 2000. Derivation of a simple clinical model to categorize patients probability of pulmonary embolism: Increasing the models utility with the SimpliRED D-dimer. Thrombosis and Haemostasis, 83(3), pp.416-420.

Whittaker, P. \& Przyklenk, K., 2009. Fibrin architecture in clots: A quantitative polarized light microscopy analysis. Blood Cells, Molecules, and Diseases, 42, pp.51-56. Available at: http://dx.doi.org/10.1016/j.bcmd.2008.10.014.

Wilhelm, M., 2002. Fourier-Transform Rheology. Macromol. Mater. Eng, 287(2), pp. 83-105.

Wilhelm, M., Maring, D. \& Spiess, H.-W., 1998. Fourier-transform rheology. Rheologica Acta, 37(4), pp.399-405.

Winter, H.H., 2002. The Critical Gel. In R. Borsali \& R. Pecora, eds. Structure and Dynamics of Polymer and Colloidal Systems. NATO Science Series, Springer, Dordrecht, the Netherlands, pp. 439-470.

Winter, H.H. \& Chambon, F., 1986. Analysis of Linear Viscoelasticity of a Crosslinking Polymer at the Gel Point. Journal of Rheology, 30, p.367. Winter, H.H. \& Mours, M., 1997. Rheology of Polymers Near Liquid-Solid Transitions. In Neutron Spin Echo Spectroscopy Viscoelasticity Rheology. Advances in Polymer Science. Berlin, Heidelberg: Springer, pp. 165-234.

Wolberg, A.S., 2012. Determinants of fibrin formation, structure, and function. Curr Opin Hematol., 19(5), pp.349-356.

Wolberg, A.S., 2007. Thrombin generation and fibrin clot structure. Blood Reviews, 21(3), pp.131-142. 
Worldthrombosisday.org, 2018. Venous Thromboembolism (VTE): The Facts. Available at: http://www.worldthrombosisday.org/issue/vte/ [Accessed October $6,2016]$.

Yao, N.Y., Larsen, R.J. \& Weitz, D.A., 2008. Probing nonlinear rheology with inertio-elastic oscillations. Journal of Rheology, 52, pp.1013-1025.

Yoshida, A., Kaburagi, Y. \& Hishiyama, Y., 2016. Scanning Electron Microscopy. In Materials Science and Engineering of Carbon Characterization. Elsevier Inc., pp. 71-93. Available at: http://linkinghub.elsevier.com/retrieve/pii/B9780128052563000052.

Zarins, C.K. et al., 1983. Carotid Bifurcation Atherosclerosis: Quantative Correlation of Plaque Localization with Flow Velocity Profiles and Wall Shear Stress.

Circulation Research, 53(4), pp.502-514. Available at: http://www.ncbi.nlm.nih.gov/pubmed/6627609.

Zhao, H. et al., 2008. Fabrication and physical and biological properties of fibrin gel derived from human plasma. Biomedical materials, 3(1), pp.1-9. 